\title{
Walddynamik in Mischwäldern des Nationalparks Hainich
}

Untersuchung der Mechanismen und Prognose der Waldentwicklung

\section{Dissertation}

\author{
zur Erlangung des Doktorgrades \\ der Mathematisch-Naturwissenschaftlichen Fakultäten \\ der Georg-August-Universität Göttingen
}

vorgelegt von

Annika Frech

aus Bielefeld

Göttingen 2006 
D 7

Referent: Prof. Dr. Christoph Leuschner

Korreferent: PD Dr. Frank Thomas

Tag der mündlichen Prüfung: 3. Mai 2006

Diese Arbeit erscheint in den Berichten des Forschungszentrums Waldökosysteme, Reihe A, Band 196, ISSN 0939-1347. 


\section{Inhaltsverzeichnis}

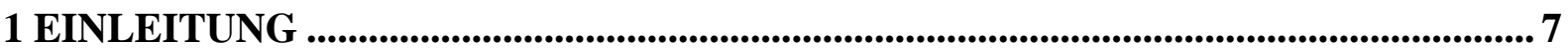

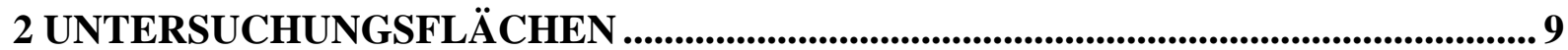

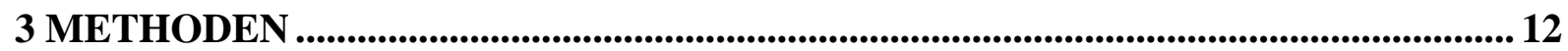

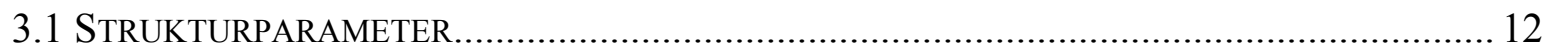

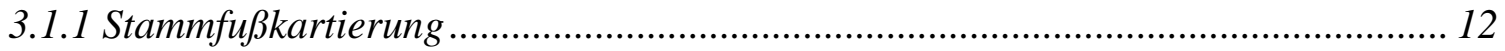

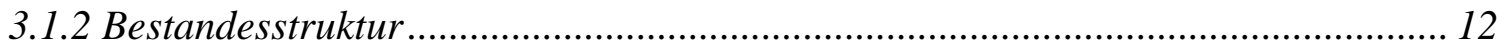

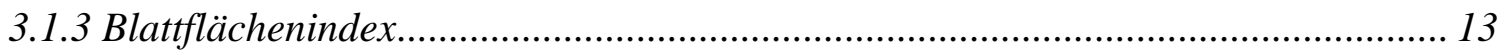

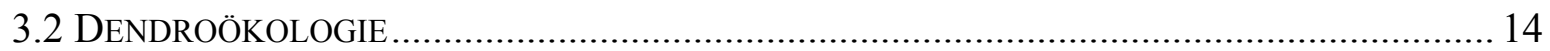

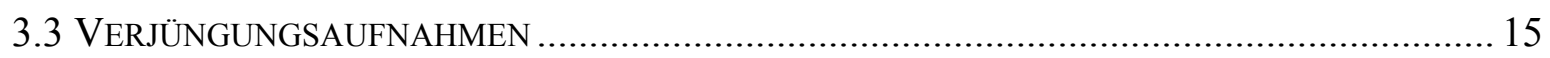

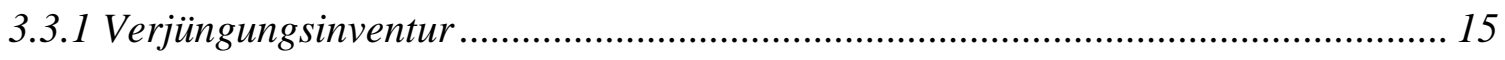

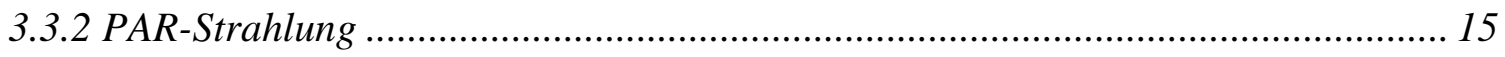

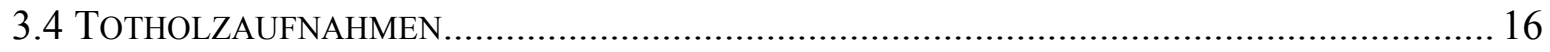

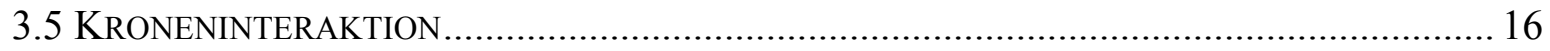

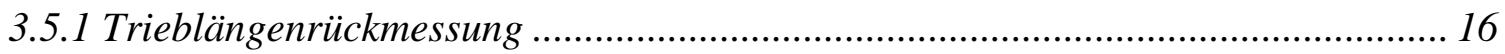

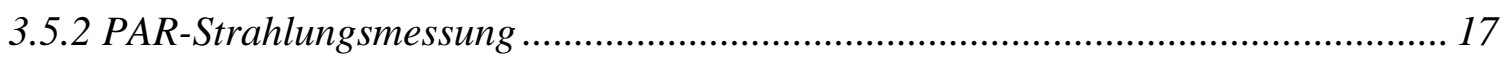

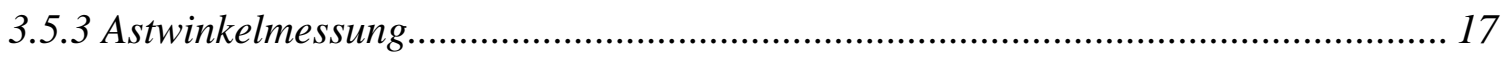

3.5.4 Länge der Kontakt-Zone...................................................................................... 18

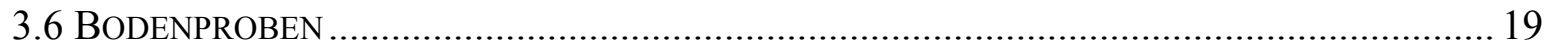

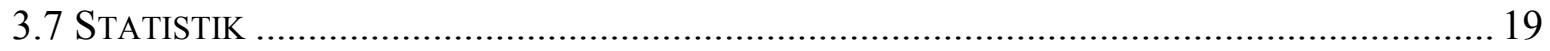

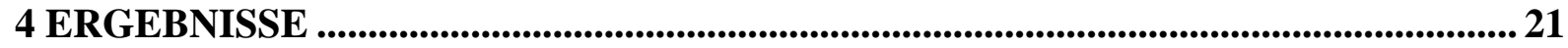

4.1 STRUKTURANALYSE DER ARTENZUSAMMENSETZUNG UND RÄUMLICHEN VERTEILUNG

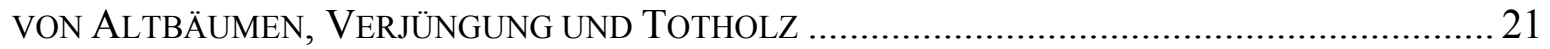

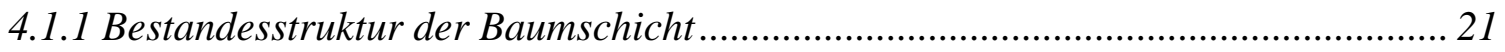

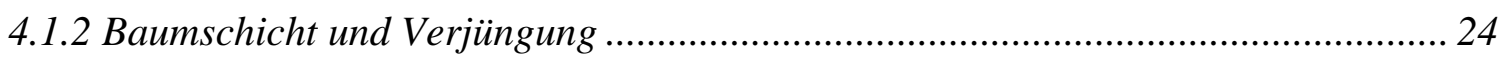

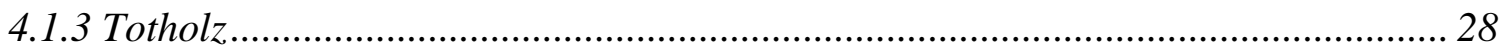

4.2 ARTSPEZIFISCHEN KRONENARCHITEKTUR UND RÄUMLICHE INTERAKTION ZWISCHEN AltBÄUMEN VON RotBuche, Esche, HAINBUCHE UND WinTERLINDE.............................. 30

4.2.1 Artspezifische Unterschiede der Kronenform ....................................................... 30 
4.2.2 Nachbarschaftsinteraktion im Kronenraum......................................................... 34

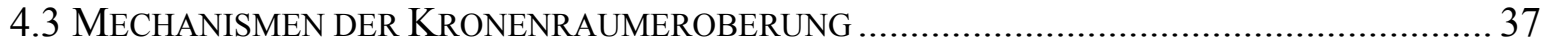

4.3.1 Astwachstum im seitlichen und oberen Kronenbereich ....................................... 37

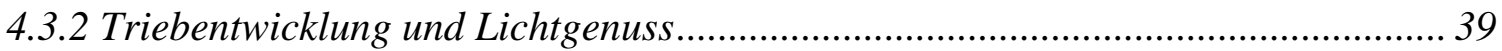

4.3.3 Triebentwicklung und mechanische Kroneninteraktion........................................ 42

4.3.4 Räumliche Interaktion - die Kontaktzone ............................................................. 44

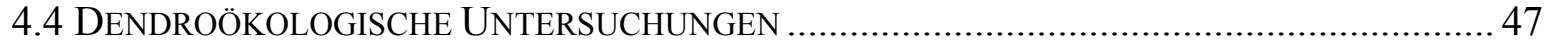

4.4.1 Stammzuwachs und Trieblängenwachstum ...................................................... 47

4.4.2 Einfluss des Klimas auf die Zuwachsdynamik........................................................ 50

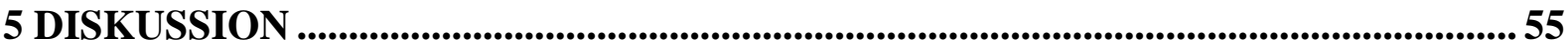

5.1 BestandesstruKtur und BESTANDESENTWICKLUNG - ARTENVERSCHIEBUNGEN ZWISCHEN TOTHOLZ, BAUMSCHICHT UND VERJÜNGUNG….................................................. 55

5.1.1 Wald- und Forstgeschichte als Ursache der heutigen Bestandesstruktur .............. 55

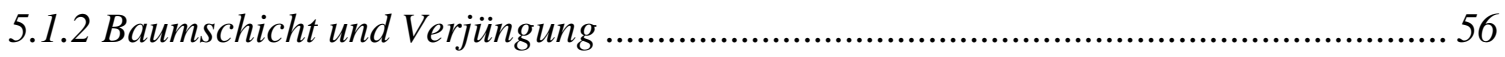

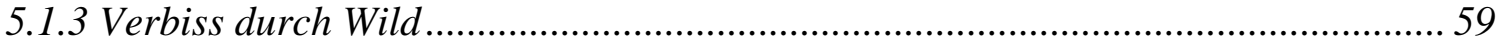

5.1.4 Regenerationsvermögen der einzelnen Arten...................................................... 61

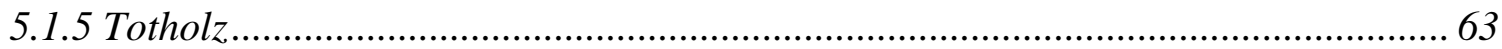

5.2 ARTSPEZIFISCHE KRONENARCHITEKTUR UND RÄUMLICHE INTERAKTION ZWISCHEN

AltBÄUMEN VON RotBuche, Esche, HAINBUCHE UND WINTERLINDE................................ 65

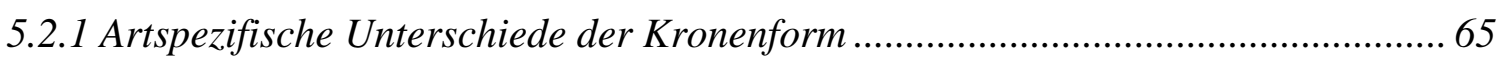

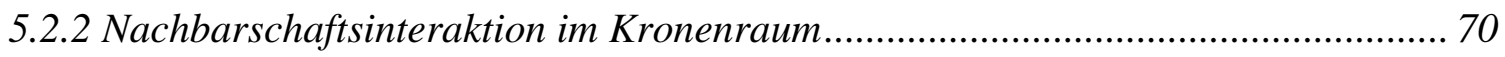

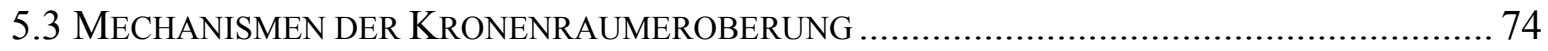

5.3.1 Baumartenspezifische Merkmale auf Astebene ........................................................ 75

5.3.2 Beschattung und Kronenkollision als Formen der Kroneninteraktion .................... 76

5.3.3 Gewichtung der Einflussgrößen auf das Trieblängenwachstum ............................. 78

5.3.4 Interaktion auf Individuenebene - die Kontaktzone ............................................. 81

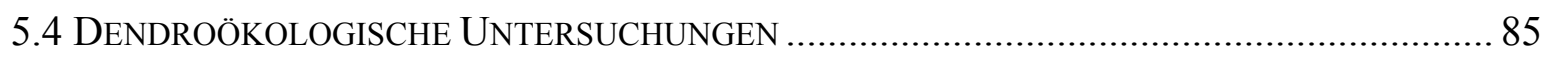

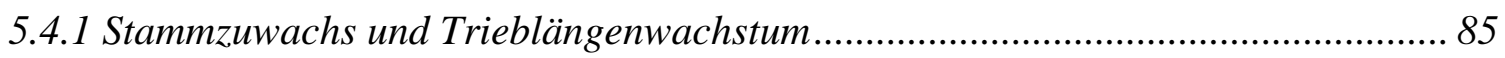

5.4.2 Einfluss des Klimas auf die Zuwachsdynamik....................................................... 86

5.5 AutÖKOlOGische Eigenschaften DER Rotbuche, Esche, HAINBUCHE UND

WINTERLINDE UND IHRE MÖGLICHE ROLLE IN DER KÜNFTIGEN WALDENTWICKLUNG.......... 92

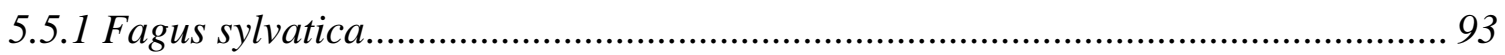

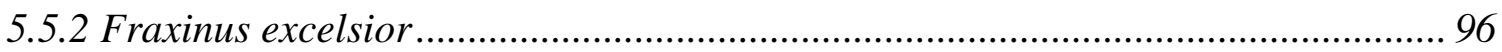




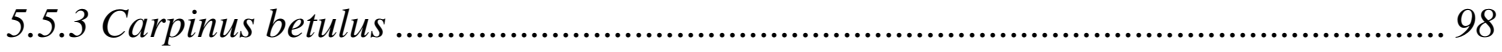

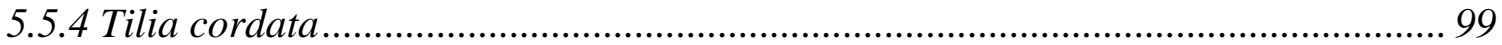

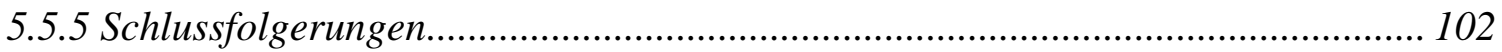

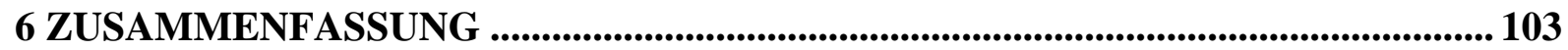

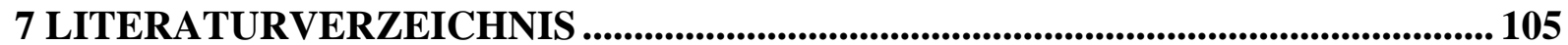




\section{Einleitung}

Die Erforschung der Struktur und Dynamik von Mischwäldern ist in den letzten Jahrzehnten verstärkt in das Interesse von Forstwirtschaft und Naturschutz gerückt, um vor dem Hintergrund der sich verändernden Umweltbedingungen (Klimawandel, Eutrophierung, Immissionsbelastung) eine Stabilisierung der Wälder und deren ökosystemaren Funktionen einschließlich der Produktivität zu gewährleisten (TARPPEINER et al.1997, ANDERSSON et al. 2004, RÖHRIG et al. 2006). Dabei werden Mischbestände mit einer standortgerechten Baumartenzusammensetzung und naturnaher Waldstruktur angestrebt. Der Realisierung dieser erklärten Ziele steht jedoch eine fehlende Kenntnis der Autökologie, der Wuchsdynamik und insbesondere der Interaktion zwischen heimischen Laubbaumarten in Mischbeständen entgegen (HUSS 1987, KENK 1992).

Im Gebiet des Nationalparks Hainich (Westthüringen) finden sich sehr baumartenreiche Laubwälder, die als beispielhaft für Mischwälder im deutschen Mittelgebirgsraum gelten können. Die aktuelle Artenzusammensetzung ist vermutlich z.T. auf menschlichen Einfluss (Mittelwaldwirtschaft) zurückzuführen, ihre natürliche Entwicklung wurde jedoch in den letzten 60 Jahren nicht mehr durch forstwirtschaftliche Eingriffe gelenkt, so dass es sich um naturnahe Mischwälder handelt, die zu Recht Teil des Nationalparks sind. In den Wäldern finden sich neben der in Mitteleuropa dominierenden Rotbuche (Fagus sylvatica L.) Esche (Fraxinus excelsior L.), Winterlinde (Tilia cordata Mill.), Hainbuche (Carpinus betulus L.), Stieleiche (Quercus robur L.), Ulme (Ulmus glabra Huds.), Berg- und Spitzahorn (Acer pseudoplatanus L., A. platanoides L.) und 3-4 weitere heimische Laubbaumarten. Diese Bestände bieten die seltene Möglichkeit, ungestörte Walddynamikprozesse in Mischwäldern zu untersuchen und wurden darum als Untersuchungsgebiet ausgewählt. Unter gleichen Standortbedingungen lassen sich hier Erkenntnisse über die artspezifische Konkurrenzstärke der wichtigsten heimischen Laubbaumarten gewinnen und forstwirtschaftlich interessante Baumartenkombinationen hinsichtlich ihrer dynamischen Entwicklung und „Kompatibilität' (Verträglichkeit) bewerten.

Den vier erstgenannten Baumarten kommt dabei eine besondere Bedeutung zu, da sie nicht nur zahlenmäßig im Gebiet überwiegen, sondern aufgrund ihrer Schattentoleranz bzw. Wuchsdynamik Schlusswaldarten sind, die langfristig das Bild dieser Wälder prägen könnten (LEIBUNDGUT 1991). 
Um Aufschluss über die Wuchsdynamik dieser Baumarten in gemischten Beständen zu erhalten, wurde im Rahmen dieser Arbeit die dreidimensionale Bestandesstruktur naturnaher artenreicher Mischbestände auf vier Untersuchungsflächen von je 0.64 ha Größe analysiert. Der gewählte Untersuchungsansatz verfolgt zwei Ziele:

1. Durch die Analyse der gegenwärtigen Bestandesstruktur einschließlich der Verjüngung und des Totholz-Vorkommens soll die Waldentwicklungsphase, in der sich die untersuchten Wälder befinden, beschrieben werden. Vor dem Hintergrund, dass es sich hier um einen Laubmischwald innerhalb des natürlichen Buchenareals handelt, soll zusätzlich der Frage nachgegangen werden, ob es mittelfristig zu einer Entmischung der artenreichen Wälder kommen wird, indem die Buche ihre natürliche Vorherrschaft zurückgewinnt.

2. Mit vertiefenden Untersuchungen zur horizontalen und vertikalen Besetzung des Kronenraums, der Nachbarschaftsinteraktion im Kronenraum (Trieblängenrückmessungen, Strahlungsmessungen und Aststellungsanalyse) und zum Wuchsverhalten durch dendroökologische Untersuchungen soll die Autökologie der vier Baumarten Rotbuche, Esche, Hainbuche und Winterlinde näher untersucht werden, um Rückschlüsse zu ziehen auf die Konkurrenzkraft und die Kompatibilität der einzelnen Baumarten im Spannungsfeld zwischen Konkurrenz und Koexistenz. 


\section{Untersuchungsflächen}

Die Untersuchungsflächen liegen im Nationalpark Hainich (Westthüringen) im südöstlichen Teil des Höhenzuges Hainich, auf dem sich das größte zusammenhängende Buchenwaldgebiet Deutschlands auf Muschelkalk erstreckt (HIEKEL et al. 2004). Die am östlichen Rand gelegenen Flächen liegen auf 290 - 300 m ü. NN in der Nähe der Ortschaft Weberstedt (s. Abb. 2.1). Es wurden vier Untersuchungsflächen von je 0.64 ha Größe in einem Radius von $3 \mathrm{~km}$ eingerichtet, auf denen die vier Baumarten Rotbuche, Hainbuche, Winterlinde und Esche in der Summe mit mindestens 80\% der Stammzahl vorhanden sind.

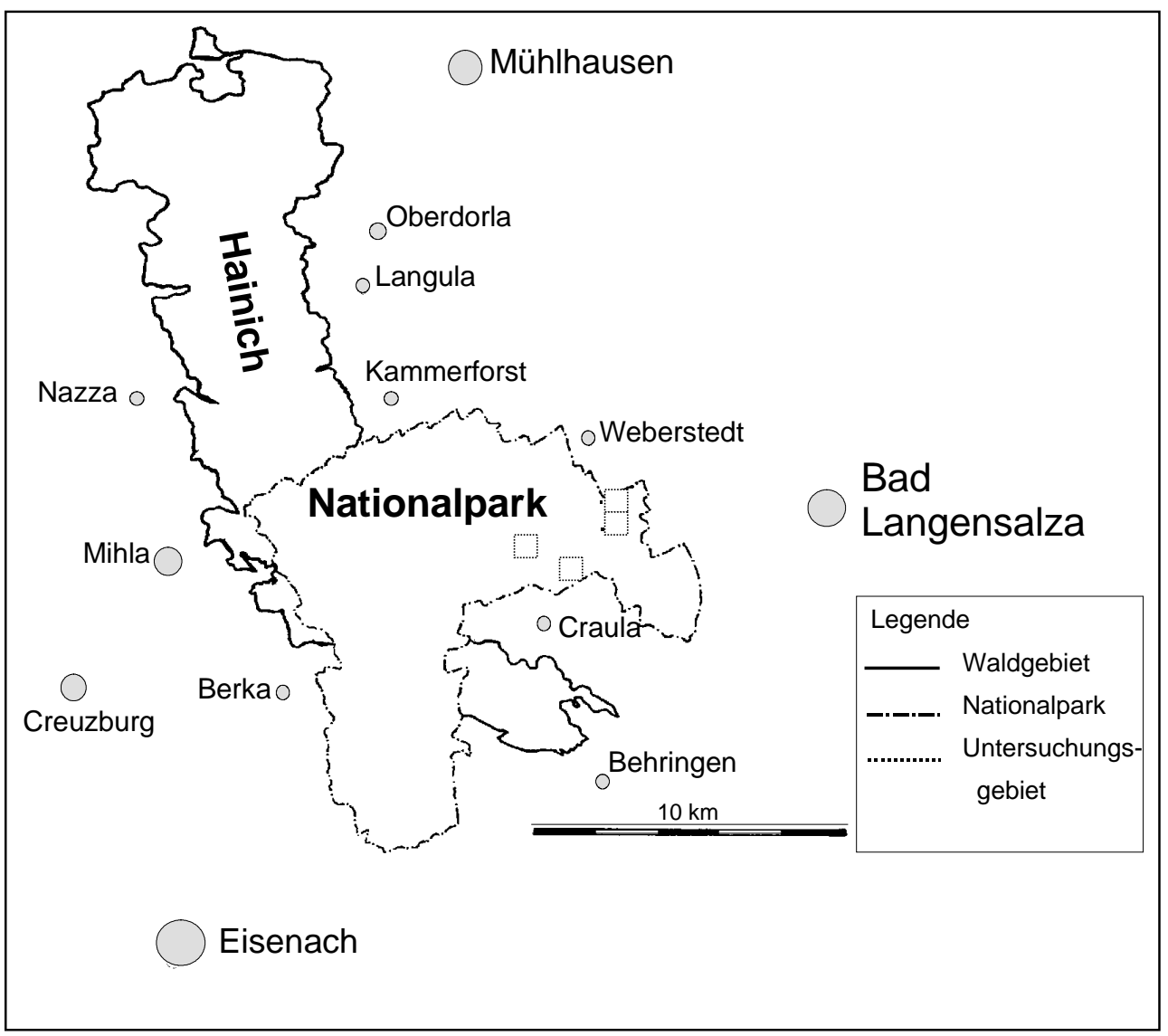

Abb.2.1: Lage des Nationalparks Hainich und der Untersuchungsflächen

Die mittlere Jahrestemperatur in Weberstedt beträgt $7.5^{\circ} \mathrm{C}$, der mittlere Jahresniederschlag liegt bei $590 \mathrm{~mm}$ (gemittelt über die Jahre 1973-2004; DEUTSCHER WETTERDIENST 2005). Klimatisch befindet sich das Gebiet im Übergangsbereich zwischen den atlantisch geprägten 
Westhängen und Hochlagen des Hainich, dem Klimabezirk „Nordwestthüringer Höhen“ innerhalb des Klimagebietes „Mitteldeutsches Berg- und Hügelland“ und dem subkontinental geprägten Klimabezirk des „Thüringer Becken“ des „Börde- und Mitteldeutschen Binnenklimas“, das am Ostrand des Hainich beginnt (HIEKEL et al. 2004).

Ausgangssubstrat für die Bodenbildung ist im Untersuchungsgebiet Mittlerer Muschelkalk, der von einer Lössschicht unterschiedlicher Mächtigkeit überlagert wird, so dass sich eine tonreiche Terra fusca-Braunerde herausgebildet hat, der ein L-Mull als Humusform aufliegt. Die mittlere Bodentiefe liegt zwischen $46.4 \mathrm{~cm}$ auf Fläche 3 und $58 \mathrm{~cm}$ auf Fläche 1. Die Mächtigkeit der Lössauflage schwankt dabei kleinräumig stark, liegt im Mittel jedoch zwischen $25.4 \mathrm{~cm}$ auf Fläche 3 und 33.6 auf Fläche 1 auf einem relativ ähnlichen Niveau. Eine detaillierte Beschreibung der Bodenbeschaffenheiten der vier Flächen gibt Burgdorf (2004).

Tab. 2.1: Ausgewählte Bodenparameter der vier Untersuchungsflächen: Aufgeführt sind die Mittelwerte der Mächtigkeit der organischen Auflage, pH-Werte, C/N-Verhältnis, effektive Kationenaustauschkapazität $\left(\mathrm{AK}_{\mathrm{e}}\right)$ und der Basensättigung sowie die Standardabweichung ( ).

\begin{tabular}{|c|c|c|c|c|c|c|}
\hline Plot & $\begin{array}{l}\text { Depth } \\
{[\mathrm{cm}]}\end{array}$ & 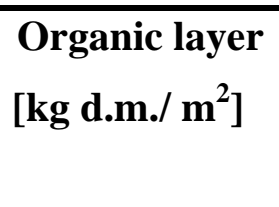 & $\mathrm{pH}_{[K C L]}$ & $\begin{array}{c}\mathrm{C} / \mathrm{N} \\
{[\mathrm{mol} / \mathrm{mol}]}\end{array}$ & $\begin{array}{c}\mathbf{A k}_{\mathrm{e}} \\
{\left[\mu \mathrm{mol}_{\mathrm{c}} / \mathbf{g} \mathrm{TB}\right]}\end{array}$ & $\begin{array}{c}\text { Base } \\
\text { saturation } \\
{[\%]}\end{array}$ \\
\hline \multirow[t]{3}{*}{1} & org. layer & $0.2(0.06)$ & $5.1(0.57)$ & $34.3(4.6)$ & & \\
\hline & $0-10$ & & $5.0(0.67)$ & $12.5(0.5)$ & 162.0 & 96.1 \\
\hline & $10-20$ & & $5.5(0.19)$ & $11.1(0.4)$ & 149.2 & 98.1 \\
\hline \multirow[t]{3}{*}{2} & org. layer & $0.2(0.05)$ & $5.6(0.18)$ & $30.2(2.9)$ & & \\
\hline & $0-10$ & & $4.6(0.52)$ & $12.3(0.8)$ & 164.4 & 97.9 \\
\hline & $10-20$ & & $4.1(0.66)$ & $10.8(0.9)$ & 117.8 & 87.4 \\
\hline \multirow[t]{3}{*}{3} & org. layer & $0.3(0.11)$ & $5.1(0.37)$ & $28.9(1.8)$ & & \\
\hline & $0-10$ & & $5.1(0.98)$ & $12.4(0.4)$ & 316.0 & 96.2 \\
\hline & $10-20$ & & $5.5(0.39)$ & $11.1(0.3)$ & 262.9 & 97.5 \\
\hline \multirow[t]{3}{*}{4} & org. layer & $0.5(0.11)$ & $5.4(0.38)$ & $26.3(2.9)$ & & \\
\hline & $0-10$ & & $4.8(0.34)$ & $12.1(0.4)$ & 205.2 & 99.8 \\
\hline & $10-20$ & & $5.0(0.45)$ & $10.5(0.5)$ & 175.8 & 99.9 \\
\hline
\end{tabular}


Eine Vegetationsaufnahme (5 x 5 m), die auf jeder Fläche an zwei Stellen am 16.04.2003 durchgeführt wurde, zeigt auf allen Flächen eine sehr ausgeprägte, artenreiche Krautschicht, die sich aus bis zu 17 verschiedene Krautarten zusammensetzt. Auf allen vier Flächen wird eine hohe Deckung zwischen 60\% (Fläche 4) und 100\% (Fläche 1) erreicht. Deckungsstärkste Kräuter waren Allium ursinum L. mit 50\%, Anemone nemorosa L. mit 50\%, Ranunculus ficaria L. mit 15\% und Deschampsia cespitosa (L.) P.BEAUV mit 10\%. Auch Leucojum vernum L. mit 2\%, Primula elatior (L.) HILL. mit 3\%, Anemone ranunculoides L. mit 2\% und Aegopodium podagraria L. mit 5\% waren zu finden. Im Laufe des Jahres wandelte sich die Artenzusammensetzung, die Gesamtdeckung ging allerdings kaum zurück. Eine eingehende Analyse der Krautschicht findet sich bei MEINEN (2004).

Pflanzensoziologisch wird das Gebiet von Ewald (2001) als Fraxinus excelsior-Fazies des Hordelymo-Fagetum eingestuft.

Ab 1961 ist das Gebiet, kurzzeitig als Naturschutzgebiet, dann als militärisches Sperrgebiet, vollständig aus der Nutzung genommen worden. Seit 1997 befindet sich der Bestand in der Schutzzone 2 des Nationalparks. 


\section{Methoden}

\subsection{Strukturparameter}

\subsubsection{Stammfußkartierung}

Das Einmessen der Stammfußpunkte erfolgte mit einem elektronischen Theodolithen, der Totalstation GTS 700. Mit Hilfe dieses Geräts wurde ausgehend von Punkten mit bereits bekannten Koordinaten unweit der Untersuchungsflächen jeweils ein Punkt in der Flächenmitte eingemessen. Von diesem aus erfolgte in einer Polarmessung die Einmessung der Stammfußpunkte sowie der Eckpunkte der Flächen. Da das Gerät nicht nur Winkel und Entfernung zu den eingemessenen Punkten ausgibt, sondern auch sofort die Gauß-KrügerKoordinaten berechnet, konnten diese ohne weitere Bearbeitung direkt in die Software des Geographischen Informationssystems (GIS) Arc-View Version 3.2 eingelesen werden. Weitere Bearbeitungsschritte wurden mit ArcGIS 9 / Arc Map vorgenommen.

\subsubsection{Bestandesstruktur}

Von allen Bäumen auf den vier Untersuchungsflächen mit einem Stammdurchmesser $\geq 7 \mathrm{~cm}$ wurde die Artzugehörigkeit bestimmt sowie der Stammumfang in $1.3 \mathrm{~m}$ Höhe gemessen und daraus der Brusthöhendurchmesser (BHD) errechnet. Als Baumartenkürzel wurde die in der Dendroökologie international anerkannte Nomenklatur verwendet (GRISSINO-MAYER \& DoBBERTIN 2003). Die Messung der Baumhöhe sowie der Höhe des Kronenansatzes erfolgte mit dem elektronischen Höhenmessgerät Vertex III der Firma Haglöf, Schweden. Dieses misst mit Hilfe von Ultraschallsignalen die Entfernung des Geräts zum Stammfußpunkt und zur Kronenspitze und errechnet automatisch über den Winkel und die Distanz zu beiden Punkten die Höhe des untersuchten Baumes.

Während der belaubungsfreien Wintermonate wurde die horizontale Ausdehnung der Baumkronen aller Bäume (BHD $\geq 7 \mathrm{~cm})$ vermessen. Für die Erfassung der Kronenprojektionsflächen diente ein Höhenmesser der Firma Suunto, Finnland, zur senkrechten Ablotung und ein Kompass zur Bestimmung der gemessenen Ausladungsrichtung. Bei den vier Zielbaumarten wurden in acht Himmelsrichtungen (N, NO, O...) die maximale Ausdehnung der Krone auf den Boden projiziert und die Entfernung dieses Punktes zum Mittelpunkt des Stammfußpunktes gemessen. Zusätzlich zu den expositionsorientierten Messungen wurden die Ausdehnungen der Krone in Richtung der Stammfußpunkte der Nachbarbäume gemessen, um die Kronendimensionen beider Nachbarn 
auf deren direkter Verbindungslinie zu erfassen. Wich die Position eines Nachbarbaums um weniger als $10^{\circ}$ von einer nach Exposition ausgerichteten Radienmessung ab, so entfiel letztere. Damit ergaben sich für jeden Messbaum mindestens 8 und maximal 12 Radienmessungen, womit nach (RÖHLE 1995) eine genügend große Anzahl von Daten zur genauen Erfassung der Kronenschirmfläche vorliegt.

Aus diesen Radien wurde nach dem von KRAMER (1987) bevorzugten Teilkreismodell die Kronenschirmfläche berechnet, die sich in diesem Rechenverfahren aus der Summe der aus dem arithmetischen Mittel zweier benachbarter Kronenradien ermittelten Kreissegmente zusammensetzt. Zusätzlich zur gesamten Kronenprojektionsfläche wurde der Anteil der direkt lichtexponierten Kronenfläche (exposed-crown area, ECA) bestimmt, die zusammen mit der relativen Höhe $(\mathrm{RH}$, im Verhältnis zur Höhe der den Kronenraum direkt begrenzenden Nachbarbäume) als wichtige Parameter für die Charakterisierung der Konkurrenzsituation (Asymmetrie/Symmetrie) in ungleich alten, gemischten Beständen angesehen wird (HIX \& LORIMER 1990, YOSHIDA \& KAMITANI 1997, 2000). Diese Zusatzmessung erwies sich als notwendig, da aus der Kronenkarte zwar die Überlappungen zu entnehmen sind, nicht jedoch, welcher der Bäume innerhalb einer Überlappungszone den anderen überschirmt. Dieser zusätzliche Wert wurde nur dann aufgenommen, wenn er um mehr als $0.5 \mathrm{~m}$ vom eigentlichen Ausladungswert abwich.

Um neben den für die weiterführenden Analysen zur Nachbarschaftsinteraktion notwendigen detaillierten Messungen auch eine vollständige Kartierung der Kronenraumbesetzung zu erreichen und visualisieren zu können, wurden auch die Kronen aller anderen auf der Fläche vorkommenden Baumarten mit vier Radienablotungen eingemessen.

Zur weiteren Beschreibung der räumlichen Arten- und Dimensionsverteilung wurden mit dem Programm BwinPro Version 6.2 (DÖBBELER ET AL. 2003) diverse Strukturindices berechnet darunter die von GADOW ET AL. (1998) eingeführten Indices Winkelmaß, Segregationsindex und Dimensionsdominanz.

\subsubsection{Blattflächenindex}

Als genaue, aber aufwändige Methode der Blattflächenbestimmung, die häufig zur Kalibrierung anderer Verfahren zur Ermittlung des Blattflächenindex (Blattfläche / Bodenfläche) dient, wurde die Aufstellung von Streufängern angewandt (NEUMANN \& DEN HARTOG 1989, Chason et al. 1991, DufrêNE \& BREDA 1995, JONCKEERE et al. 2004). Ab Mitte August wurden auf jeder Fläche 30 Streufänger mit einer Öffnungsweite von $0.29 \mathrm{~m}^{2}$ aufgestellt. Die Streufänger wurden unmittelbar neben den randomisiert ausgewählten Kleinflächen zur Verjüngungsuntersuchung auf vier Transekten pro Fläche angeordnet (s. 
Kap. 3.3) und alle vier Wochen geleert, bis der Laubfall endgültig abgeschlossen war. Die Blätter wurden für jeden Streufänger gesondert nach Arten sortiert und bei $60{ }^{\circ} \mathrm{C}$ vier Tage getrocknet. Anschließend wurde das Trockengewicht für jede Baumart in jedem Streufänger bestimmt. Jeweils 20 Blätter pro Art und Fläche wurden vor dem Trocknen gescannt, um mit dem Programm WinFolia (Version 5.1) die Blattflächen zu ermitteln. Anschließend wurde über das spezifische Blattgewicht auf die gesamte Blattfläche im Bestand hochgerechnet und der Blattflächenindex (Leaf-Area-Index LAI) für jede Fläche ermittelt.

\subsection{Dendroökologie}

Für die dendroökologischen Untersuchungen wurden von jeder der vier untersuchten Arten pro Fläche fünf Individuen ausgewählt, die eine herrschende Stellung im Bestand einnehmen und deren BHD mindestens $35 \mathrm{~cm}$ beträgt. Unter den in Frage kommenden Bäumen wurde randomisiert ausgewählt. Bäume, die sich näher als $5 \mathrm{~m}$ am Flächenrand befanden wurden ausgeschlossen, um Randeffekte auszuschließen.

Die Bohrkerne wurden mit zwei Zuwachsbohrern der Firma Suunto (Finnland) auf $1.3 \mathrm{~m}$ Höhe entnommen (SchweINGRUBER 1996). Pro Baum wurde ein Bohrkern gezogen. Das Bohrloch wurde anschließend mit einem Rundholz verschlossen und mit künstlicher Rinde abgedichtet.

Für die Jahrringanalyse wurden die Bohrkerne angeschliffen und zunächst unter dem Binokular untersucht. Anschließend wurden die Jahrringbreiten mit Hilfe eines beweglichen Messtisches, dessen Bewegungen der angeschlossene Computer elektronisch erfasst, vermessen und mit dem Softwareprogramms TSAP (Tree Samples Analysis Program Version 3.0; RINN 1996) weiter analysiert. Für die Untersuchung der Klimasensitivität wurde durch cross-dating (NORTON \& OGDEN 1990) je eine baumartenspezifische Mittelkurve erstellt, die aus mindestens 15 Individuen errechnet wurde, die bei mehr als hundert Jahren eine Gleichläufigkeit von mindestens 65 \% mit der Mittelkurve aufwiesen. Da die Gleichläufigkeit stark von der Überlappungslänge der Kurven abhängt, muss die Gleichläufigkeit mit abnehmender Überlappung zunehmen, um eine zufällige Lage von der korrekten Synchronlage zu unterscheiden. Dieser Wert steigt nach ECKSTEIN \& BAUCH (1969) auf 70\%, wenn die Überlappung auf 50 Jahre abnimmt. Zur weiteren statistischen Absicherung wurden Korrelationen nach BAILLE \& PILCHER (1973) und HoLlSTEIN (1980) berechnet. 


\subsection{Verjüngungsaufnahmen}

\subsubsection{Verjüngungsinventur}

Auf allen vier Untersuchungsflächen wurde auf jeweils 40 Subplots von $1 \mathrm{~m}^{2}$ Größe eine Jungbauminventur nach Aufnahmeverfahren von PRETZSCH (2002) durchgeführt. Die Kleinflächen lagen randomisiert auf jeweils vier Nord-Süd-ausgerichteten Transekten von 60 m Länge, deren Anfangspunkte teilrandomisiert im nördlichen Viertel jeder Fläche mit einem Mindestabstand von $5 \mathrm{~m}$ zueinander und zum Rand festgelegt wurden. Mit Ausnahme der Keimlinge des Vorjahres wurden alle Jungbäume, die größer als $5 \mathrm{~cm}$ waren und einen geringeren Brusthöhendurchmesser als $7 \mathrm{~cm}$ aufwiesen, erfasst. Bei jedem Jungbaum wurde die Art, die Höhe bis zur höchsten Leittriebknospe und der Wurzelhalsdurchmesser (WHD) in $5 \mathrm{~cm}$ Höhe aufgezeichnet. Ein Mindestalter wurde nach der Triebmorphologie für Buchen nach ROLOFF (2001) anhand der Jahreseinschnürungen geschätzt und auch auf alle anderen Baumarten angewendet. Zusätzlich wurde die Häufigkeit des Leittriebverbisses durch Wild nach vier Schädigungsklassen differenziert aufgenommen (Kein Verbiss: 0, einmaliger Leittriebverbiss: 1, mehrmaliger Leittriebverbiss: 1m, Totalverbiss/ Jungbaum ohne Blätter: $t$ ). An den vier Hauptbaumarten wurden zusätzlich eine vollständige Trieblängenrückmessung vorgenommen, wenn diese höher als $10 \mathrm{~cm}$ waren.

\subsubsection{PAR-Strahlung}

Die Strahlungsmessungen wurden mit PAR-Sensoren der Firma LICOR (Lincoln, Nebraska, USA) vom Typ LI-190SA, die jeweils vor der Messsaison gegeneinander kalibriert wurden, durchgeführt. Die Sensoren erfassen die Photonenstromdichte (Einheit: $\mu E \cdot \mathrm{m}^{-2} \cdot \mathrm{s}^{-1}$ ) im photosynthetisch wirksamen Bereich des Lichtspektrums, d. h. Wellenlängen zwischen 400 und $700 \mathrm{~nm}$. Die Messwerte wurden in einem angeschlossenen Data-Logger LI 1000 von der Firma Licor (Lincoln, Nebraska, USA) gespeichert. Ein Referenz-Sensor war im Freiland ca. $100 \mathrm{~m}$ außerhalb des Bestandes in $1.80 \mathrm{~m}$ Höhe angebracht. Dort wurde alle zehn Sekunden ein Wert gemessen und als Mittelwert über eine Minute gespeichert. Beide Sensoren waren exakt zeitlich aufeinander abgestimmt. Eine kardanische Aufhängung stellte eine immer horizontale Ausrichtung sicher. Im Bestand betrug die Messhöhe ca. 1.20 m. Der Sensor war an einer $2 \mathrm{~m}$ langen Stange befestigt, um einen ausreichenden Abstand zur Messperson zu gewährleisten und so Schattenbildung durch die Messenden zu vermeiden. Jeder Plot wurde geviertelt und über jedem Mittelpunkt jedes Quandranten und dem Mittelpunkt des gesamten Plots fünf Wiederholungsmessungen durchgeführt. 


\subsection{Totholzaufnahmen}

Die Totholz-Aufnahmen orientierten sich an den methodischen Vorgaben von PRETZSCH 2002 und MEYER et al. 2000). Demnach wurde nur das Derbholz aufgenommen, das einen Durchmesser von mindestens $7 \mathrm{~cm}$ und eine Mindestlänge von $2.5 \mathrm{~m}$ aufwies. Es wurde in liegendes, stehendes und hängendes Totholz unterteilt; Stubben wurden ebenfalls aufgenommen. Beim stehenden und hängenden Totholz wurde jeweils der BHD und die Höhe bestimmt, beim liegenden der Durchmesser am dickeren Ende und ein zweiter Durchmesser nach $3 \mathrm{~m}$ sowie die Gesamtlänge aufgenommen. Bei Stubben wurde der Durchmesser und die Höhe gemessen. Soweit möglich, wurde die Artzugehörigkeit anhand von Rindenresten und Holzstruktur bestimmt und auch, ob es sich um Stammteile oder Äste handelte. Die Mortalitätsursache war nur in den wenigsten Fällen festzustellen.

Der Zersetzungsgrad wurde in Anlehnung an ALBRECHT (1991) in vier Klassen unterteilt. Für Stämme und Stubben, die bereits so stark zersetzt waren, dass eine Volumenschätzung kaum mehr möglich war, wurde eine weitere Klasse, Zersetzungsgrad 4+, eingeführt.

Um die räumliche Lage der aufgenommenen Totholzstücke zu erfassen, wurde jeweils der Winkel und der Abstand des dickeren Stammendes zum nächsten lebenden Nachbarbaum gemessen.

Für die Kubierung wurde für Stämme, Stammteile und Starkäste die Hubertsche Formel (KRAMER 1987) zur Volumenbestimmung herangezogen. Stubben wurden als Neiloidstumpf berechnet.

\subsection{Kroneninteraktion}

\subsubsection{Trieblängenrückmessung}

Die Untersuchungen zur Kroneninteraktion wurden in drei aufeinanderfolgenden Vegetationsperioden nach Abschluss der Triebwachstumsphase und vor Beginn der Blattseneszenz durchgeführt. Die Messungen wurden mit Hilfe einer Hebebühne vom Typ DL30 der Firma DENKA LIFT durchgeführt, die einen direkten, störungsarmen Zugang zum obersten Kronenraum ermöglicht (maximale Auslegung $30 \mathrm{~m}$ ).

Am Vergleich der Trieblängen zwischen Ästen in der direkten 'Kontaktzone', die ein Individuum mit einem Nachbarbaum teilt, und frei stehenden Wipfeltrieben lässt sich feststellen, wie stark die individuelle laterale Wuchseinschränkung ist. Dazu wurden pro Individuum jeweils an fünf Wipfeltrieben und fünf Trieben in jeder erreichbaren Kontaktzone 
im seitlichen Kronenraum die Trieblängen der letzten sechs Jahre vermessen. Unterhalb der sechsten Zuwachseinheit (ZE) wurde der Astumfang gemessen.

Es wurden nur die Terminaltriebe von Starkästen vermessen (1.-5. Ordnung), die zum 'Gerüst' bzw. Kronenmantel gehören (GLEISSNER 1998), da die Lebenserwartung und das Längenwachstum mit höherer Astordnung abnimmt (SUZUKI 2002) ist und Seitenachsen häufiger Infloreszenzen tragen.

Es wurde mit erfasst, ob die Triebabschnitte aus der Terminalknospe eines Triebes hervorgegangen sind oder aus einer Seitenknospe. So lassen sich Zweigabsprünge und Abbrüche quantifizieren, die vor allem durch Wind und 'Kronenkollisionen' entstehen (ROLOFF 2001).

Ebenfalls aufgenommen wurde die Blatt- und Knospenzahl des diesjährigen Triebes sowie die Anzahl der Lang- und Kurztriebe eines jeden Triebabschnitts (ZE).

\subsubsection{PAR-Strahlungsmessung}

An allen Triebspitzen wurde die relative Beleuchtungsstärke (in \% der Freilandstrahlung) der photosynthetisch aktiven Strahlung (PAR) im Wellenlängenbereich zwischen 400 und $700 \mathrm{~nm}$ gemessen um festzustellen, wie stark die Beschattung durch die Nachbarkrone im Vergleich zur mechanischen Kroneninteraktion für eine laterale Wuchseinschränkung verantwortlich ist. Die Messungen wurden mit zwei PAR-Sensoren der Firma Licor (Lincoln, Nebraska, USA) vom Typ LI-190SA durchgeführt, die jeweils an Datalogger der gleichen Firma (Typ LI1000) angeschlossen waren. Für die punktgenaue Messung oberhalb der Triebspitzen wurde der Sensor in eine Fassung eingeschraubt, die an einer Kardanischen Aufhängung angebracht war. Diese war an einen Verlängerungsstab montiert, so dass auch an schwer zugänglichen Stellen im Kronenraum eine exakt horizontale Ausrichtung des Sensors gewährleistet war. Zur Ermittlung des Referenzwertes im Freiland diente ein Sensor, der ca. $10 \mathrm{~m}$ vor dem nahe gelegenen Waldrand in $1.8 \mathrm{~m}$ Höhe angebracht war.

\subsubsection{Astwinkelmessung}

Zusätzlich zu den Trieblängen wurde die räumliche Stellung der Triebe mit einem Gerät gemessen, das für Blattwinkelmessungen entwickelt wurde. Es handelt sich um einen neu entwickelten „vollkompensierten elektronischen Kompass 3d ODS“, der in der Forstwissenschaftlichen Fakultät (Institut für Waldbau I) zusammen mit Technikern der Firma Saxotec entwickelt wurde und bislang nur als Prototyp existiert. Das Gerät misst simultan die Himmelsrichtung, die Steigung und die seitliche Neigung und wird über ein entsprechendes Programm von einem Laptop aus bedient. Als Kooperationspartner dieses 
Projektes stellte Herr Prof. Dr. von Lübke als Leiter des Instituts für Waldbau I dieses Messgerät dankenswerter Weise zur Verfügung.

Gemessen wurde jeweils die sechste, am weitesten innen liegende Zuwachseinheit, um die Hauptwuchsrichtung des Astes festzulegen. Nach außen hin wurde dann jeder zweite Jahresabschnitt eingemessen. Zusätzlich wurde jeder Triebabschnitt aufgenommen, dessen Wuchsrichtung um mehr als $20^{\circ}$ von dem vorhergehenden abwich. Mittels Vektorrechnung wurde schließlich die kürzeste Strecke zwischen dem Anfangs-Messpunkt (an der Basis der sechsten Zuwachseinheit) und der Treibspitze errechnet und in Beziehung zur aufsummierten Trieblänge gesetzt.

\subsubsection{Länge der Kontakt-Zone}

$\mathrm{Da}$ sich beim Vermessen der Trieblängen zeigte, dass die Bereiche, in denen zwei benachbarte Bäume aneinander grenzen je nach Art auf sehr unterschiedlichen Höhenniveaus liegen, wurden zusätzlich zu den Arbeiten auf Ast- und Zweigebene die Länge und die absolute Höhe im Bestand der jeweiligen „Kontakt-Zonen“ vermessen.

Die Kontaktzone zwischen zwei Altbäumen ist definiert als der Bereich, in dem die Bäume jeweils mit ihrem beblätterten Kronenmantel aneinander angrenzen. Die genaue Bezeichnung sollte demnach „beblätterte Kontaktzone“ sein. Nur dort kann ein gegenseitiges aktives Ausweichen vor, bzw. Eindringen in die Nachbarkrone stattfinden.

Um die Vergleichbarkeit zu gewährleisten, wurden nur Kontakt-Zonen vermessen, die seitlich nicht in eine Kronenlücke übergehen, da diese ein seitliches Ausweichen der Kronen möglich machen, die die direkte Konkurrenz um Raum und Licht im Kronenraum mindern.

Die vertikale Ausdehnung wurde jeweils vom obersten Punkt der Kontakt-Zone ausgelotet sowie die vertikale Distanz von ihrer Obergrenze zu den Wipfeln der beteiligten Bäume bestimmt. Bei nicht durchgehenden Kontaktzonen wurden vertikale Lücken von über $4 \mathrm{~m}$ gesondert mit aufgenommen und die daraus entstehenden vertikalen Zonen einzeln gemessen. Zusätzlich zur vertikalen Längenmessung erfolgte eine Klassifizierung der horizontalen Ausdehnung der Kontakt-Zonen, die die Intensität des Eindringens der Kronen ineinander charakterisiert. Berücksichtigt wurden nur die Bereiche, in denen beide Bäume gleichermaßen Blätter trugen. Überschirmungen in vertikaler Schichtung wurden nicht als gemeinsamer Kronenraum angesehen.

Die Art des Kronenkontaktes wurde in folgende Klassen eingeteilt:

1. Kronenscheu: Kronen berühren sich bei Windstille nicht, sondern lassen einen deutlichen Spalt $(>20 \mathrm{~cm})$ zwischen sich frei. 
2. Kronen grenzen direkt aneinander an und berühren sich dabei nur leicht (partielle Überlappung bis $50 \mathrm{~cm}$ ).

3. Kronen greifen ineinander mit Überschneidungsbereichen von mehr als $50 \mathrm{~cm}$.

\subsection{Bodenproben}

Die Bodenprobennahme erfolgte an jeweils fünf zufällig ausgewählten Punkten pro Fläche, wobei darauf geachtet wurde, dass der Abstand zum nächstgelegenen Baum mindestens $1.5 \mathrm{~m}$ betrug. Die Entnahme der organischen Auflage zur Bestimmung des Humusvorrats erfolgte mit einem 30x30 cm-Stechrahmen. Die weitere Probenahme fand in den Tiefen von 0 bis 10 $\mathrm{cm}$ und 10 bis $20 \mathrm{~cm}$ statt, wobei in beiden Bodentiefen der Mineralboden dreimalig mit einem $100 \mathrm{~cm}^{3}$-Zylinder beprobt und die Einzelproben $\mathrm{zu}$ einer Mischprobe vereinigt und nach Entfernung von Ästen und Zweigen bei $4^{\circ} \mathrm{C}$ eingelagert wurden. Die pH-WertMessungen wurden mit einer pH-Gaselektrode (Sentix 41) durchgeführt. Die Bestimmung der Konzentration von Kohlenstoff und Stickstoff erfolgte gaschromatographisch mit einem Elementaranalysator (C/N-Analyser vario EL III).

Um die Tiefgründigkeit des Bodens sowie die Mächtigkeit der Lössauflage und der Tonschicht zu bestimmen, wurde außerdem an jeder Probestelle ein Einschlag mit dem Pyrckhauer-Bohrer vorgenommen.

\subsection{Statistik}

Die statistischen Analysen und Tests in der vorliegenden Arbeit sind mit dem Datenverarbeitungssystem SAS (Statistical Analyses System, SAS Institute Inc., Cary, NC, USA) durchgeführt worden.

Die Stichprobenreihen wurden zunächst auf Normalverteilung untersucht; zu diesem Zweck wurde ein Anpassungstest von SHAPIRO \& WILK benutzt. Mit Stichprobenreihen, die sich größtenteils als normalverteilt erwiesen, wurde mit einer einfaktoriellen Varianzanalyse untersucht, ob die unabhängige Variable einen signifikanten Einfluss auf die Gesamtvarianz des Parameters hat. Für alle Datensätze wurde einheitlich auf dem Signifikanzniveau $p \leq 0.05$ geprüft.

Bei nicht normalverteilten Probenreihen wurde ein nicht-parametrischer Mittelwertsvergleich nach WILCOXON, MANN und WHITHNEY (U-Test) durchgeführt, der analog zur einfaktoriellen Varianzanalyse testet, ob der Einfluss der unabhängigen Variable auf die Varianz des Parameters signifikant ist. Anschließend wurde ein Rangsummentest durchgeführt. 
Neben diesen Analysen wurden verschiedene Regressionen berechnet, bei denen das Programm Xact (Version 10) der Firma SciLab GmbH verwendet wurde.

Zur Durchführung multifaktorieller Regressionsanalysen wurde das Programm SPSS (Version 12) verwendet. 


\section{Ergebnisse}

\subsection{Strukturanalyse der Artenzusammensetzung und räumlichen Verteilung von Altbäumen, Verjüngung und Totholz}

\subsubsection{Bestandesstruktur der Baumschicht}

Ein wichtiges Kriterium für die Wahl der Untersuchungsflächen war neben einer grundsätzlichen Vergleichbarkeit der klimatischen und edaphischen Bedingungen eine ähnliche Bestandesstruktur bei möglichst hoher Durchmischung der Baumarten.

Mit Stammzahlen zwischen 359 Bäumen pro Hektar auf Fläche 1 und 413 auf Fläche 4 weisen die Flächen eine sehr ähnliche Bestandesdichte auf. Die Basalflächen liegen zwischen $34.01 \mathrm{~m}^{2} /$ ha (Fläche 3) und $40.99 \mathrm{~m}^{2} /$ ha (Fläche 2) auf (s. Tab.4.1).

Tab. 4.1: Vergleich der vier Untersuchungsflächen anhand der Parameter Stammzahl und Grundfläche pro Hektar sowie durch Index-Werte zur Charakterisierung der Artenvielfalt und der räumlichen Verteilung der Arten auf den Flächen

\begin{tabular}{l|cccc}
\hline Study Plot & P1 & P2 & P3 & P4 \\
\hline Number of tree species & 12 & 10 & 10 & 8 \\
Stem density [n/ha] & 359 & 384 & 402 & 413 \\
Basal area [m²/ha] & 35.23 & 40.99 & 34.01 & 36.53 \\
Evenness (G) & 0.76 & 0.68 & 0.79 & 0.76 \\
Mingling Index (M) & 0.59 & 0.55 & 0.67 & 0.44 \\
Spatial distribution Index (W) & 0.53 & 0.54 & 0.56 & 0.55 \\
Index Clark \& Evans (R) & 0.93 & 0.94 & 0.95 & 0.88 \\
Vertical distribution $\left(\mathrm{A}_{\text {rel }}\right)[\%]$ & 66.6 & 80.0 & 76.5 & 69.0 \\
\hline
\end{tabular}

Zur Beschreibung der Artdurchmischung und räumlichen Verteilung im Bestand wurden mit dem Programm BwinPro (DöBBELER ET AL. 2003) verschiedene Indizes berechnet, die einen ersten Eindruck von der Bestandesstruktur vermitteln. Die Evenness gibt als standardisierter Shannon-Index (korrigiert nach Donelly 1978) einen Wert zwischen 0 und 1, der die Diversität eines Bestandes beschreibt, in dem er Artenvielfalt und Abundanz der Arten miteinander in Beziehung setzt. Er berücksichtigt die Tatsache, dass ein Mischbestand umso vielfältiger ist, je mehr Arten vertreten sind und dass die Diversität mit abnehmender Variabilität in den Baumartenanteilen ebenfalls zunimmt (PIELOU 1977). Die 
Standardisierung ermöglich dabei eine Vergleichbarkeit der Diversität mit anderen Beständen trotz unterschiedlicher Artenzahlen. Plot 3 erweist sich mit einem Wert von 0.79 als am stärksten durchmischt. Durch die Standardisierung erscheinen die Bestände P1 und P4 mit einem Evenness-Wert von 0.76 gleich in ihrer Diversität, obwohl auf Fläche 1 zwölf, auf Fläche 4 hingegen nur acht Baumarten vertreten sind, da auf beiden Flächen die Artendurchmischung ähnlich hoch ist. Der Durchmischungsindex (M) nach FÜLDNER (1995), der auf das Inventurverfahren der ,Strukturellen Vierergruppe' zurückgeht und angibt, wie viele der drei nächsten Nachbarn eines Baumes der gleichen Art angehören, berücksichtigt diesen Unterschied in der Artenvielfalt und zeigt für P1 mit 0.59 einen deutlich höheren Wert als für P4 (0.44). Ein Index-Wert von 0 gib an, dass alle Nachbarbäume der gleichen Art angehören, bei 0.33 sind zwei von drei Nachbarn konspezifisch, bei 0.66 nur einer. Mit einem Wert von 0.67 für P 3 zeigt sich diese als optimal durchmischt, wohingegen sich auf Fläche 4 eine Tendenz zur Bildung kleiner, artreiner Gruppen abzeichnet.

Die Indizes von Clark \& Evans (R) sowie das Winkelmaß (W) geben ausschließlich Auskunft über das Stammverteilungsmuster. Der Index von CLARK UND EVANS (1954) beschreibt die räumliche Verteilung der Individuen auf der Fläche, indem der mittlere berechnete Abstand zwischen einem Baum und seinem nächsten Nachbarn mit dem mittleren zu erwartenden Abstand bei Zufallsverteilung ins Verhältnis gesetzt wird. Werte um 1 zeigen eine Zufallsverteilung an, was auf den Flächen 1 bis 3 mit Werten zwischen 0.93 und 0.95 gegeben erscheint. Werte $<1$ zeigen eine zunehmende Tendenz zur Klumpung. Mit 0.88 liegt Fläche 4 noch im Bereich der Poisson-Verteilung, weicht allerdings etwas stärker vom Richtwert $1 \mathrm{ab}$ als die anderen Flächen. Das Winkelmaß (W) (GADOW et al. 1998; ALBERT 1999) beschreibt analog zum Durchmischungsindex (M) die Regelmäßigkeit der Verteilung der drei nächsten Nachbarn um den Bezugsbaum. Werte zwischen 0.5 und 0.56 zeigen eine zufällige Verteilung an. Alle Flächen weisen einen Index-Wert innerhalb dieser Spanne auf.

Eine wichtige Zusatzinformation liefert der von PRETZSCH (1996) entwickelte Index zur vertikalen Artenmischung. Der Artprofil-Index (A) baut auf dem Index von SHANNON (1948) auf und wird umso größer, je mehr Baumarten in einem Waldbestand vorkommen und je intensiver sich diese vertikal im Bestand vermischen. Für einschichtige Reinbestände nimmt A den Wert 0 an. Der standardisierte Artprofilindex $A_{\text {rel }}$ gibt in Prozent an, wie nah ein Bestand bei gegebener Artenzahl einer maximal möglichen Strukturierung kommt, wie sie in einem ungleichaltrigen Mischbestand gegeben ist. Dieser Wert liegt für Fläche 2 mit 80\% am höchsten, gefolgt von Fläche 3 (76.5\%). Fläche 1 und 4 fallen mit 66.6\%, bzw. 69\% der maximal möglichen vertikalen Durchmischung dagegen leicht ab. 
Insgesamt lässt sich festhalten, dass alle Flächen bei relativ ähnlicher Bestandesdichte und zufälliger Stammverteilung eine hohe Artenvielfalt und eine gute Durchmischung aufweisen, wobei Fläche 3 die höchsten Index-Werte für die Durchmischung aufweist und somit besonders homogen in der Artenmischung erscheint.

Diese Homogenität zeigt sich für Fläche 3 auch im Vergleich der mittleren Stammstärken. Hier zeigen sich keine signifikanten Unterschiede zwischen den Baumarten (s. Abb. 4.1). Die mittleren Brusthöhendurchmesser (DBH) liegen zwischen $13.6 \mathrm{~cm}$ (Hainbuche; in der Abbildung mit Cabe abgekürzt) und $25.7 \mathrm{~cm}($ Esche $=$ Frex $)$.

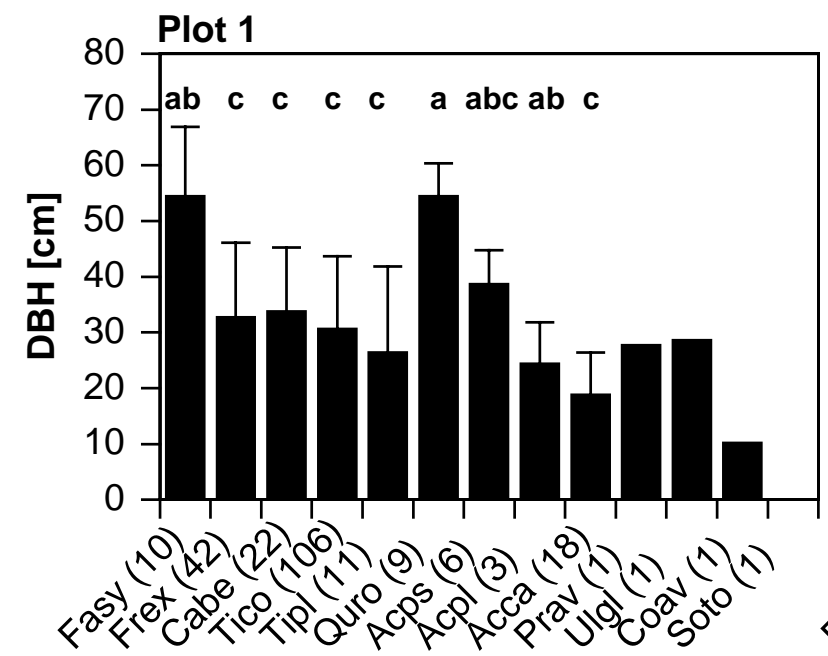

Plot 2
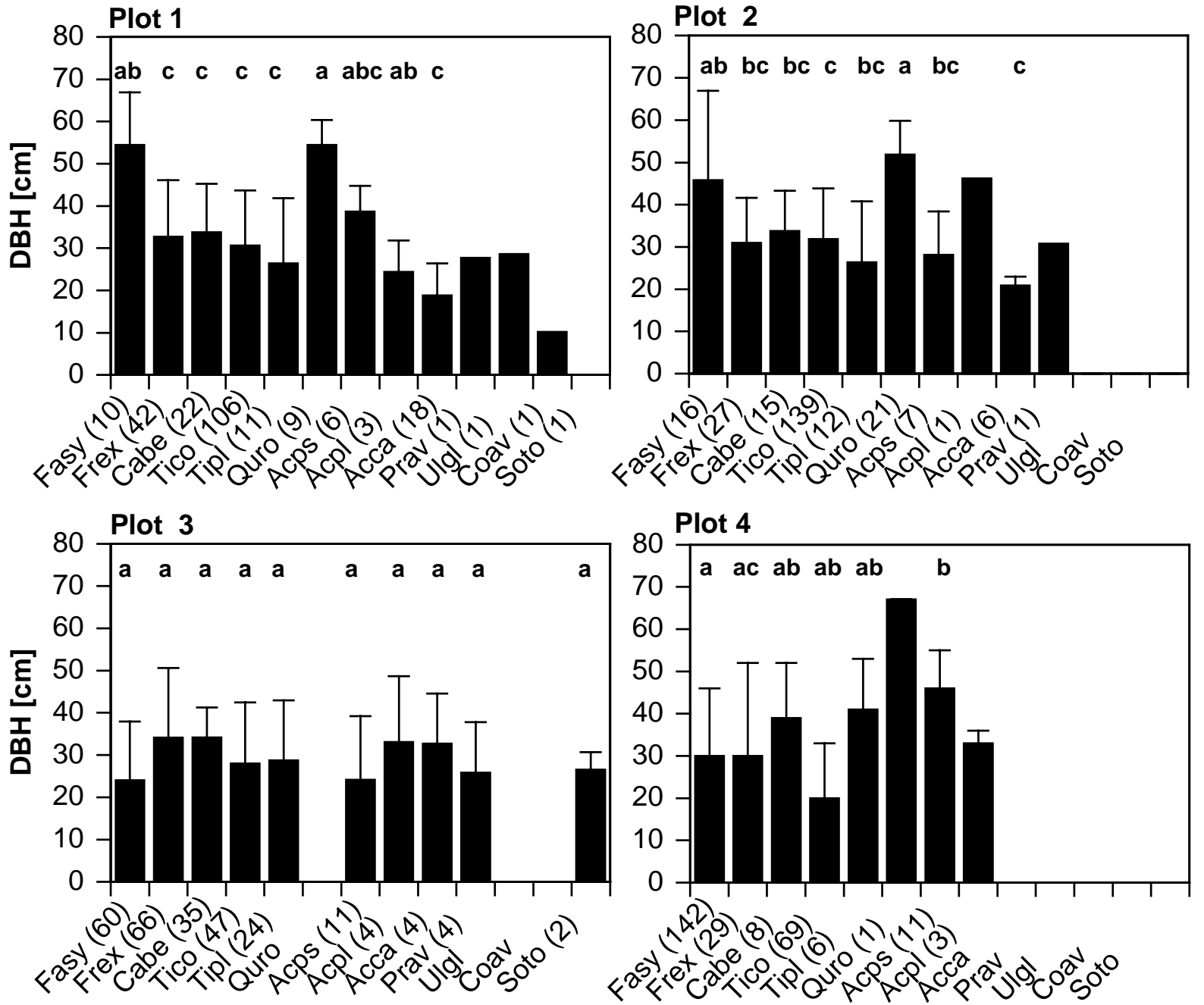

Abb. 4.1: Mittlere Brusthöhendurchmesser aller vorkommenden Arten auf den vier Untersuchungsflächen. Die Werte hinter den Baumartenkennungen geben die absolute Häufigkeit der Art auf der Fläche an $($ Fasy $=$ Fagus sylvatica $\mathrm{L}$. Frex $=$ Fraxinus excelsior L., Cabe $=$ Carpinus betuls L., Tico $=$ Tilia cordata MILL., Tipl = Tilia platyphyllos SCOP., Quro = Quercus robur L., Acps $=$ Acer pseudoplatanus L. $=$ Acpl = Acer platanoides L., Acca $=$ Acer campestre L., Prav $=$ Prunus avium L., $\mathrm{Ulgl}=$ Umus glabra HUDS. em MOSS, Soto = Sorbus torminalis (L.) CR., Coav = Corylus avellana L. Unterschiedliche Kleinbuchstaben kennzeichnen signifikante Unterschiede zwischen den Arten einer Fläche(MANN and WHITNEY U-test; $\mathrm{p} \leq 0.05$ ). 
Charakteristisch für Fläche 1 und 2 sind die großen Durchmesser von Buche $(54.5 \mathrm{~cm}$ auf Fläche 1 und $45.8 \mathrm{~cm}$ auf Fläche 2) und Eiche (54.5 cm, bzw. $51.9 \mathrm{~cm})$, die sich signifikant von der mittleren Stammstärke der dominierenden Winterlinde unterscheiden. Auf Fläche 4 ist der mittlere Stammdurchmesser der Winterlinden mit $20.0 \mathrm{~cm}$ signifikant geringer als der der dominierenden Baumart Buche $(30.5 \mathrm{~cm})$ und der Hainbuche $(39.0 \mathrm{~cm})$.

Mit der Verschiebung der Dominanzverhältnisse von Winterlinde auf den Flächen 1 und 2 über die gleichmäßig durchmischte Fläche 3 hin zur buchendominierten Fläche 4 geht eine Änderung der relativen Beleuchtungsstärke am Waldboden einher. Mit einer im Vergleich zum Freiland um 98.7\% herabgesenkten Strahlungsintensität ist Fläche 4 die dunkelste Fläche (s. Abb. 4.2). Sie weist zugleich mit 8.25 den höchsten Blattflächenindex (LAI) und damit die stärkste Belaubungsdichte auf. Die am stärksten lindendominierte Fläche 2 weist einen signifikant geringeren LAI auf (6.4) und ist dem entsprechend mit $2.3 \%$ der relativen Beleuchtungsstärke um ein Prozent heller als die Buchen-Fläche 4.

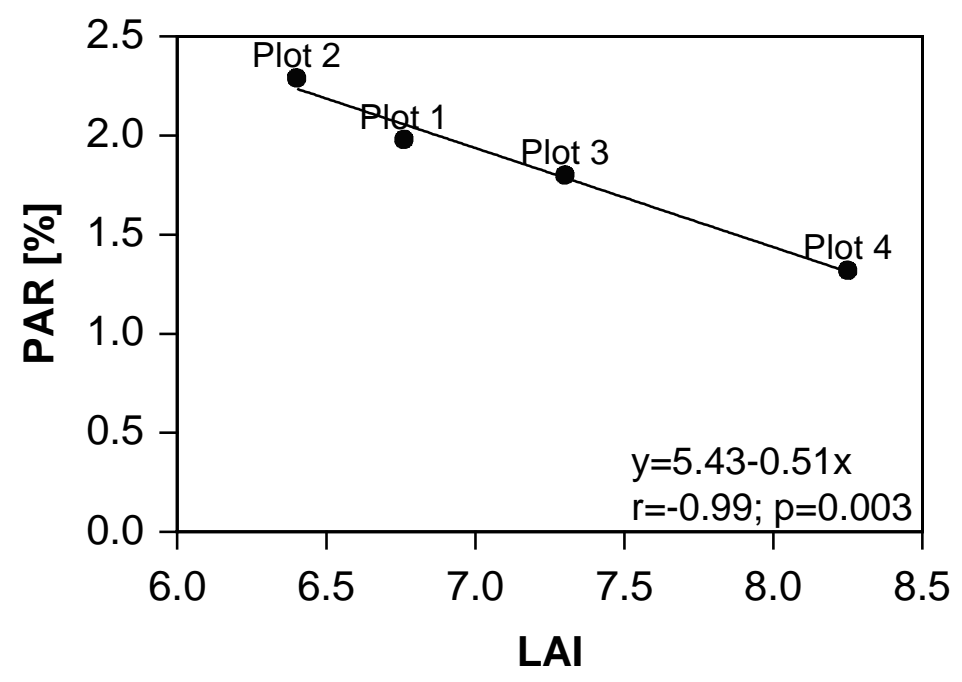

Abb. 4.2: Relative Beleuchtungsstärke (PAR) am Waldboden in Abhängigkeit vom Blattflächenindex (LAI) der vier Untersuchungsflächen.

\subsubsection{Baumschicht und Verjüngung}

Auf allen Flächen sind die Arten Rotbuche (Fasy), Esche (Frex), Hainbuche (Cabe), Winterlinde (Tico), Sommerlinde (Tipl), Bergahorn (Acps) und Spitzahorn (Acpl) in der Baumschicht vorhanden, jedoch mit sehr unterschiedlichen Anteilen. Auf den Flächen 1 und 2 dominiert die Winterlinde deutlich mit 47.1\%, bzw. 57.5\% der relativen Häufigkeit (s. Abb. 4.3). Auf Fläche 3 ist die Abundanz der Arten Buche (23.4\%), Esche (25.7\%) sehr ähnlich, auch Winterlinde mit $18.3 \%$ und Hainbuche mit 13.6\% sind relativ stark vertreten. Auf Fläche 
4 dominiert eindeutig die Buche mit 52.8\%. Nur die Winterlinde mit 25.6\% und Esche mit 10.8\% kommen noch mit einer Häufigkeit von über 10\% vor. Die Ahornarten sind auf allen Flächen nur mit weniger als 5\% vertreten. Eine Ausnahme bildet lediglich der Feldahorn auf Fläche 1 mit $7.83 \%$.

Die Abundanzverhältnisse in der Baumschicht spiegeln sich in der Häufigkeitsverteilung der Arten in der Verjüngung nicht wider (s. Abb. 4.3). Hier herrschen andere Arten vor. Auf Fläche 1 und 2 dominieren Eschen im Jungwuchs mit 57.2\% und 65.4\%. Auf Fläche 3 ist diese Art nur mit 15.7\% vertreten, obwohl sie hier in der Baumschicht viel häufiger vorkommt als auf allen anderen Flächen. Auf Fläche 4 ist sie hingegen wieder dominant. Die in der Baumschicht auf Plot 1 und 2 vorherrschende Winterlinde ist auf diesen Flächen nur mit 1.1\%, bzw. 0.2\% in der Verjüngung repräsentiert. Auch auf den Flächen 3 und 4 erreicht sie nur $2.8 \%$ und $1.7 \%$.
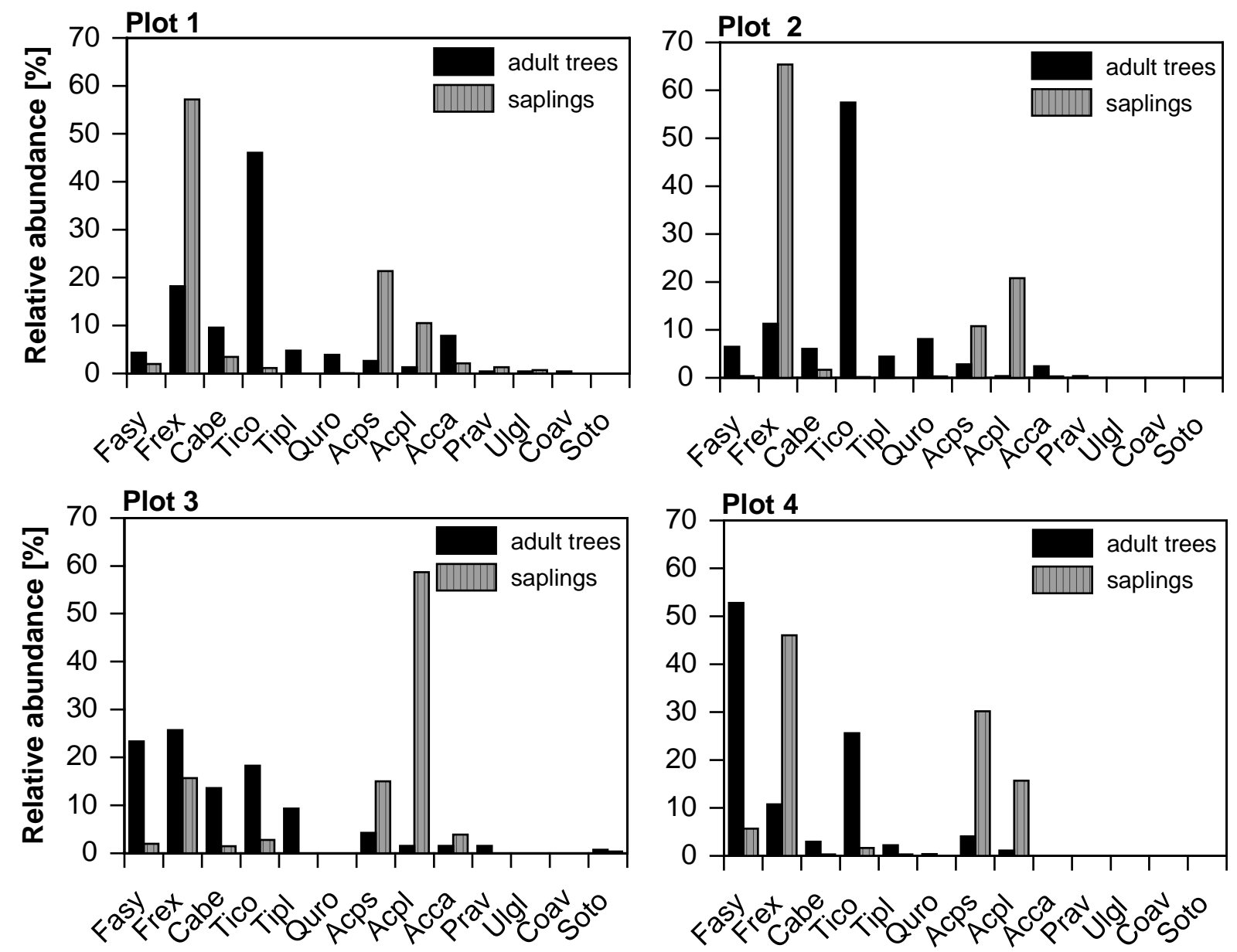

Abb. 4.3: Häufigkeitsverteilung der Baumarten in der Baumschicht (schwarze Balken) und in der Verjüngung (graue Balken) (Baumartenkennung s. Abb. 4.1 oder Abkürzungsverzeichnis)

Auch die Buche ist in der Verjüngung nur sehr schwach vertreten. Selbst auf der buchendominierten Fläche 4 beträgt ihr Anteil an der gesamten Verjüngung nur 5.7\%. Auf 
Fläche 1 ist diese Art mit 0.4\% kaum vertreten. Ausgehend von den Artabundanzen in der Baumschicht, ist der große Anteil der Ahornarten am Jungwuchs erstaunlich. Auf Fläche 3 dominiert Spitzahorn mit 57.8\%, zusammen mit Bergahorn kommt der Anteil an Ahorn an Jungbäumen auf 72.8\% auf dieser Fläche. Auch auf den anderen Flächen ist der Ahorn-Anteil mit 45.9\% auf Fläche 4 und 31\% auf den Flächen 1 und 2 sehr hoch.

Winterlinden sind in der Verjüngung im Vergleich zu ihrer Häufigkeit in der Baumschicht selten. Die wenigen vorhandenen Jungbäume erreichen aber eine signifikant größere Höhe und weisen einen größeren Wurzelhalsduchmesser auf als alle anderen Arten (s. Abb. 4.4).
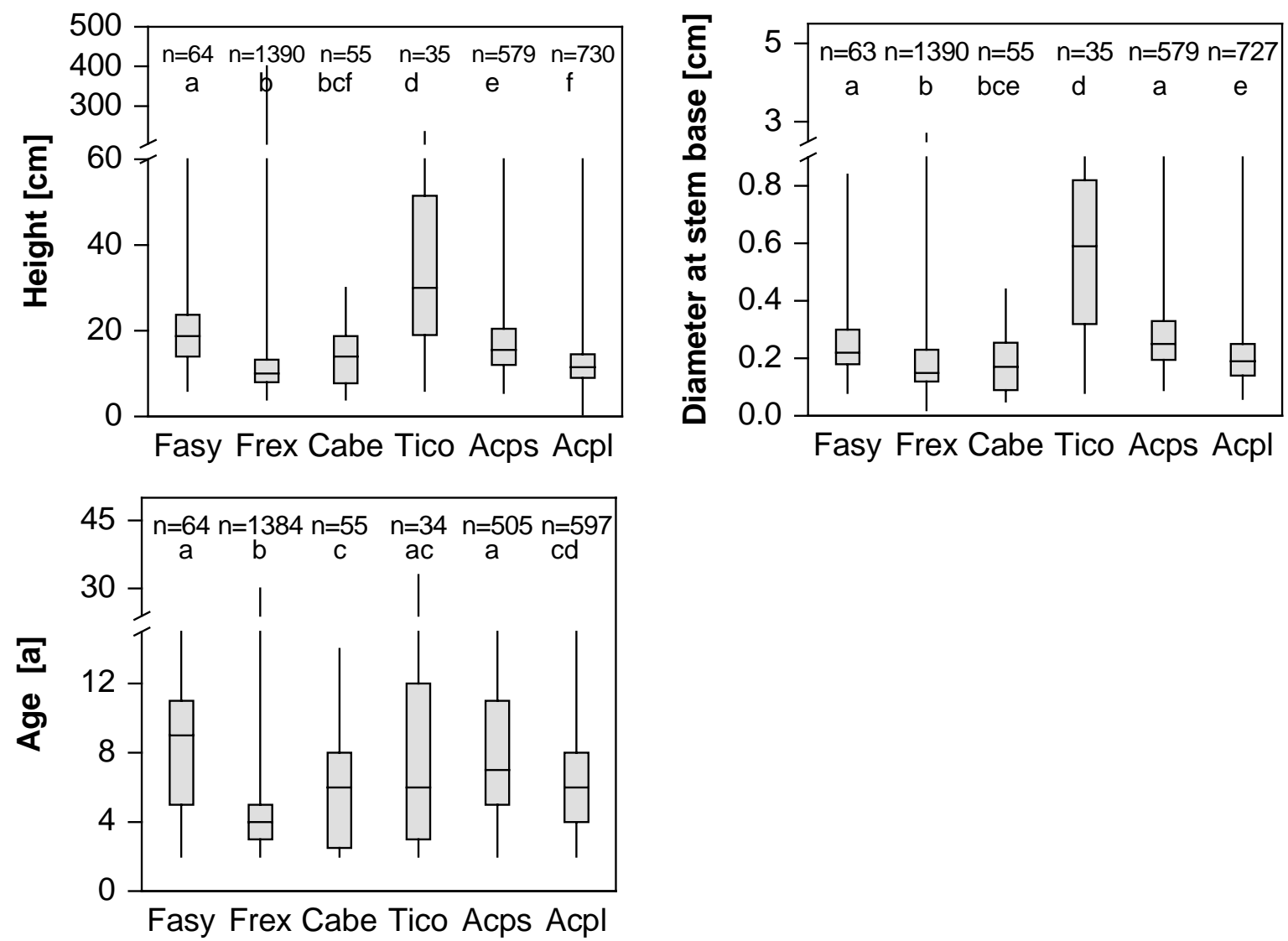

Abb. 4.4: Höhe, Wurzelhalsdurchmesser und Altersverteilung der wichtigsten Baumarten in der Verjüngung auf den vier Untersuchungsflächen dargestellt in Box-Whisker-Plots. Der Querstrich innerhalb der Box gibt den Median an. Unterschiedliche Kleinbuchstaben kennzeichnen signifikante Unterschiede zwischen den Arten (U-Test nach MANN \& WHITNEY).

Die ältesten Jungbäume auf den untersuchten Kleinflächen sind ebenfalls Linden, jedoch unterscheidet sich das durchschnittliche Alter des Lindenjungwuchses nicht von dem der Buchen und Bergahorne. Die zahlenmäßig dominierende Esche ist mit einigen wenigen großen Individuen in der Verjüngung vertreten im Durchschnitt weist sie jedoch die signifikant geringste Höhe und Stammdicke und das niedrigste Alter auf. 
Tab. 4.2: Relative Häufigkeit des Wildverbisses an den wichtigsten Baumarten in der Verjüngung. Unterschiedliche Kleinbuchstaben geben statistisch signifikante Unterschiede zwischen den Arten an (Chi-Quadrat-Test).

\begin{tabular}{l|lc}
\hline Species & \multicolumn{2}{|l}{$\begin{array}{l}\text { Saplings } \\
\text { by game bite [\%] }\end{array}$} \\
\hline Fasy & 61.90 & $\mathrm{ac}$ \\
Frex & 43.12 & $\mathrm{~b}$ \\
Cabe & 50.91 & $\mathrm{cb}$ \\
Tico & 80.00 & $\mathrm{a}$ \\
Acps & 72.51 & $\mathrm{a}$ \\
Acpl & 72.35 & $\mathrm{a}$ \\
\hline
\end{tabular}

Eschen haben aber auch die geringste Verbissrate (s. Tab. 4.2). Nur 43\% der Jung-Eschen zeigen Verbiss-Spuren, während bei beiden Ahornarten die Rate über $72 \%$ liegt. Am stärksten ist die Winterlinde mit $80 \%$ vom Wildverbiss betroffen.

Bei den Verjüngungsaufnahmen wurde stets mit erfasst, welcher Art der überschirmende Altbaum über der jeweiligen Kleinfläche angehörte. Eine Übersicht über die Verteilung der einzelnen Arten im Jungwuchs unter die Schirme der verschiedenen Altbaumarten gibt

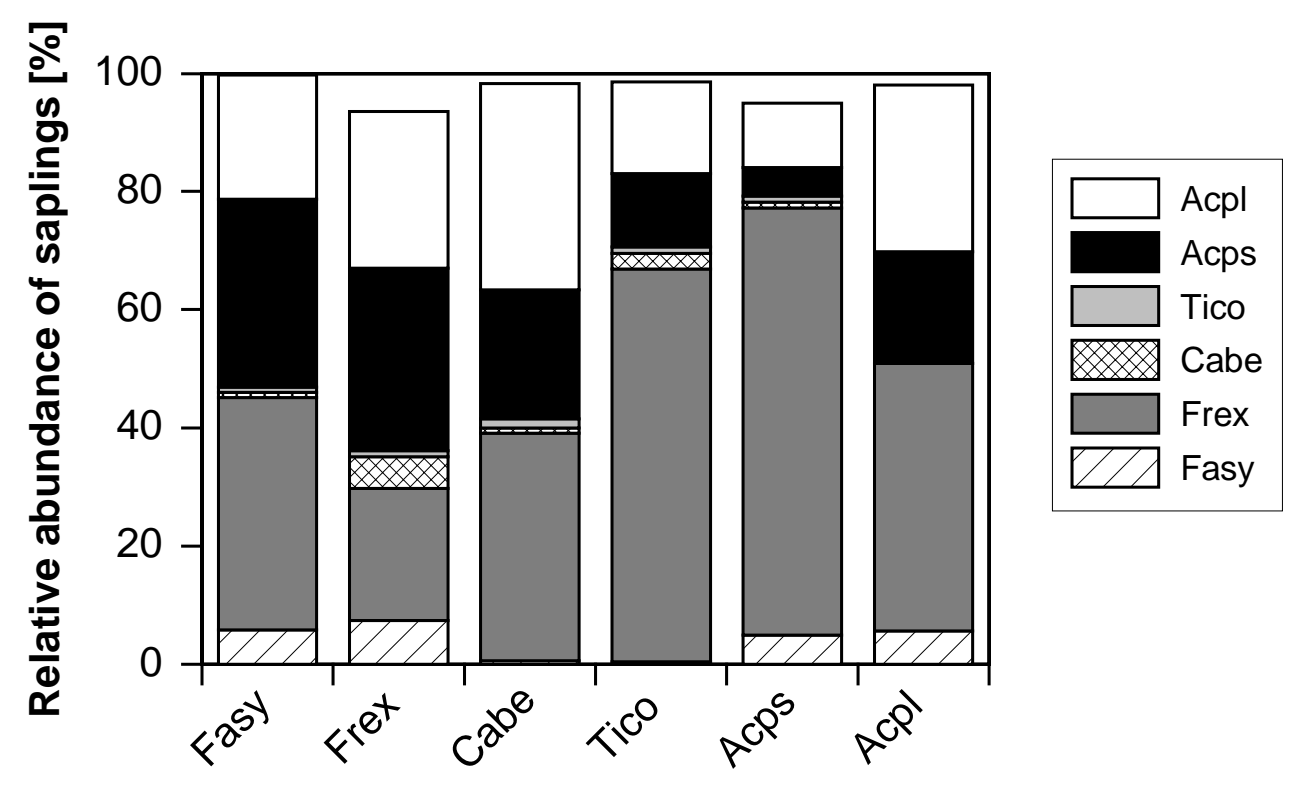

Species in the canopy layer

Abb. 4.5: Relative Häufigkeit der Verjüngung der einzelnen Arten unter dem Schirm der häufigsten Baumarten der Kronenschicht. 
Abbildung 4.5. Die am stärksten Jungwuchs aufweisenden Arten Esche (grau Balken), Bergahorn (schwarze Balken) und Spitzahorn (weiße Balken) waren unter allen Altbäumen anzutreffen. Auffällig ist, dass Esche und Bergahorn das prozentual geringste Auftreten unter gleichartigen Altbäumen aufweisen. Unter Eschen verjüngt sich Bergahorn mit 30.8\% am häufigsten, wohingegen unter Bergahornschirm mit 72.3\% Esche im Jungwuchs dominiert. Mit 66.4\% aller Jungbäume nimmt die Esche den größten Raum unter den Winterlinden der Baumschicht ein. Auch unter Buchen- und Hainbuchenschirm ist sie mit 39.3\%, bzw. 38.5\% die dominierende Art im Jungwuchs. Hier sind aber auch die Ahornarten ebenfalls häufig vertreten. Diese Unterschiede in der Häufigkeitsverteilung der Esche erwiesen sich allerdings als nicht signifikant (Wilkoxon-U-Test). Die Verteilung der Jungpflanzen unter dem Altbaum-Schirm erwies sich für alle Arten als zufällig. Lediglich das sehr geringe Auftreten von Buchenverjüngung unter Hainbuchen und Winterlinde zeigte einen statistisch nachweisbaren Unterschied: Buchenjungpflanzen waren signifikant häufiger unter Bergahornund Buchen-Schirm als unter Hainbuche und Winterlinde.

\subsubsection{Totholz}

Die Vorkommen von totem Derbholz sind sehr unterschiedlich hoch auf den vier Untersuchungsflächen. Sie schwanken zwischen $4.81 \mathrm{~m}^{3} \mathrm{ha}^{-1}$ auf Fläche 4 und $25.59 \mathrm{~m}^{3} \mathrm{ha}^{-1}$ auf Fläche 2 (Tab. 4.3). Relativ ähnlich hingegen ist der Anteil an stehendem Totholz, der auf allen Flächen rund 50\% beträgt.

Tab. 4.3: Gesamtvolumina des Totholzvorkommens auf den vier Untersuchungsflächen

\begin{tabular}{c|ccc}
\hline Plot & $\begin{array}{c}\text { Volume of dead } \\
\text { wood } \\
{\left[\mathbf{m}^{\mathbf{3}} / \mathbf{\text { Plot}}\right]}\end{array}$ & $\begin{array}{c}\text { Volume of dead } \\
\text { wood } \\
{\left[\mathbf{m}^{\mathbf{3}} / \mathbf{h a}\right]}\end{array}$ & $\begin{array}{c}\text { Percentage of standing } \\
\text { dead wood } \\
{[\%]}\end{array}$ \\
\hline 1 & 5.71 & 8.96 & 51.6 \\
2 & 16.38 & 25.59 & 51.83 \\
3 & 8.03 & 12.55 & 48.4 \\
4 & 3.08 & 4.81 & 42.5 \\
\hline
\end{tabular}

Ein differenzierteres Bild des Totholzvorkommens gibt Abb. 4.6. Der im Vergleich hohe Totholzvorrat auf Fläche 2 setzt sich zusammen aus erst kürzlich abgestobenen Buchen (schwarze Balken zeigen den Anteil an Totholz der Zerfallsklasse $1=$ noch nicht sichtbar zersetzt) und einem großen Eichen-Anteil von $4.3 \mathrm{~m}^{3}$, der der Zerfallsklasse 2 zugeordnet ist. 


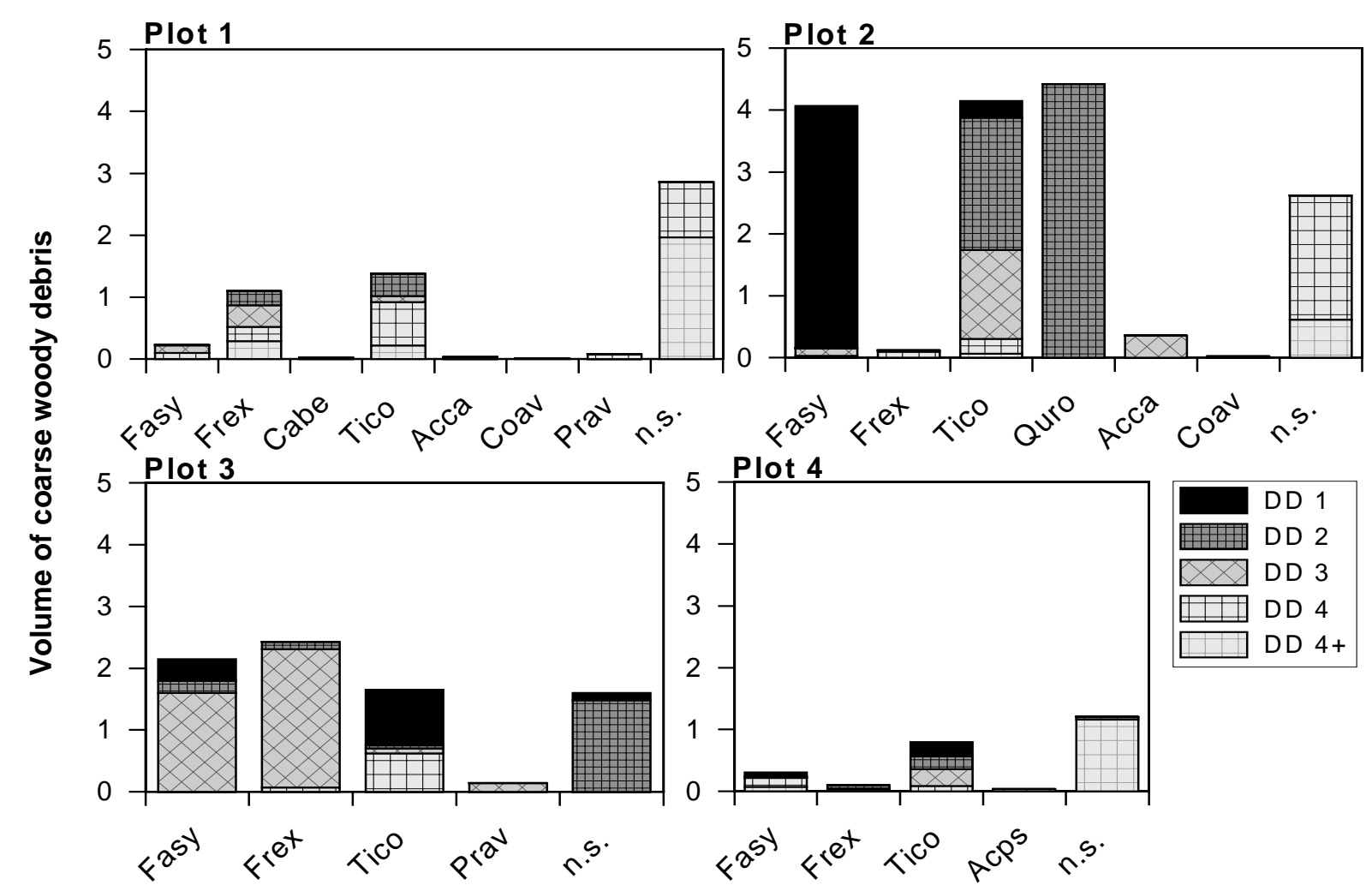

Abb. 4.6: Volumen des toten Derbholzes der einzelnen Baumarten auf den vier Untersuchungsflächen differenziert nach Zersetzungsgraden (DD) von DD1 = kaum zersetzt bis D4+ = stark zersetzt.

Der Zeitpunkt des Absterbens liegt dem entsprechend etwas länger zurück. Hinzu kommt ein mit $4.2 \mathrm{~m}^{3}$ ähnlich hoher Anteil an Winterlinde, der sich jedoch über alle Zerfallsklassen erstreckt. Auf Fläche 3 setzt sich das gefundene Totholz vor allem aus Esche und Winterlinde zusammen, auch Winterlinde ist mit $1.8 \mathrm{~m}^{3}$ vertreten. Auf der buchendominierten Fläche 4 hat die Winterlinde mit $0.8 \mathrm{~m}^{3}$ den größten Anteil des Totholzanteils, bei dem eine Artansprache eindeutig möglich war. Ein großer Anteil entfällt hier auf stark zersetztes Totholz, dessen Artzugehörigkeit nicht mehr ermittelbar war. 


\subsection{Artspezifischen Kronenarchitektur und räumliche Interaktion zwischen} Altbäumen von Rotbuche, Esche, Hainbuche und Winterlinde

\subsubsection{Artspezifische Unterschiede der Kronenform}

Für die Analyse der räumlichen Struktur und der Aufteilung des Kronenraums auf den vier Untersuchungsflächen wurden die Altbäume der Arten Fagus sylvatica, Fraxinus excelsior, Tilia cordata und Carpinus betulus eingehender untersucht.

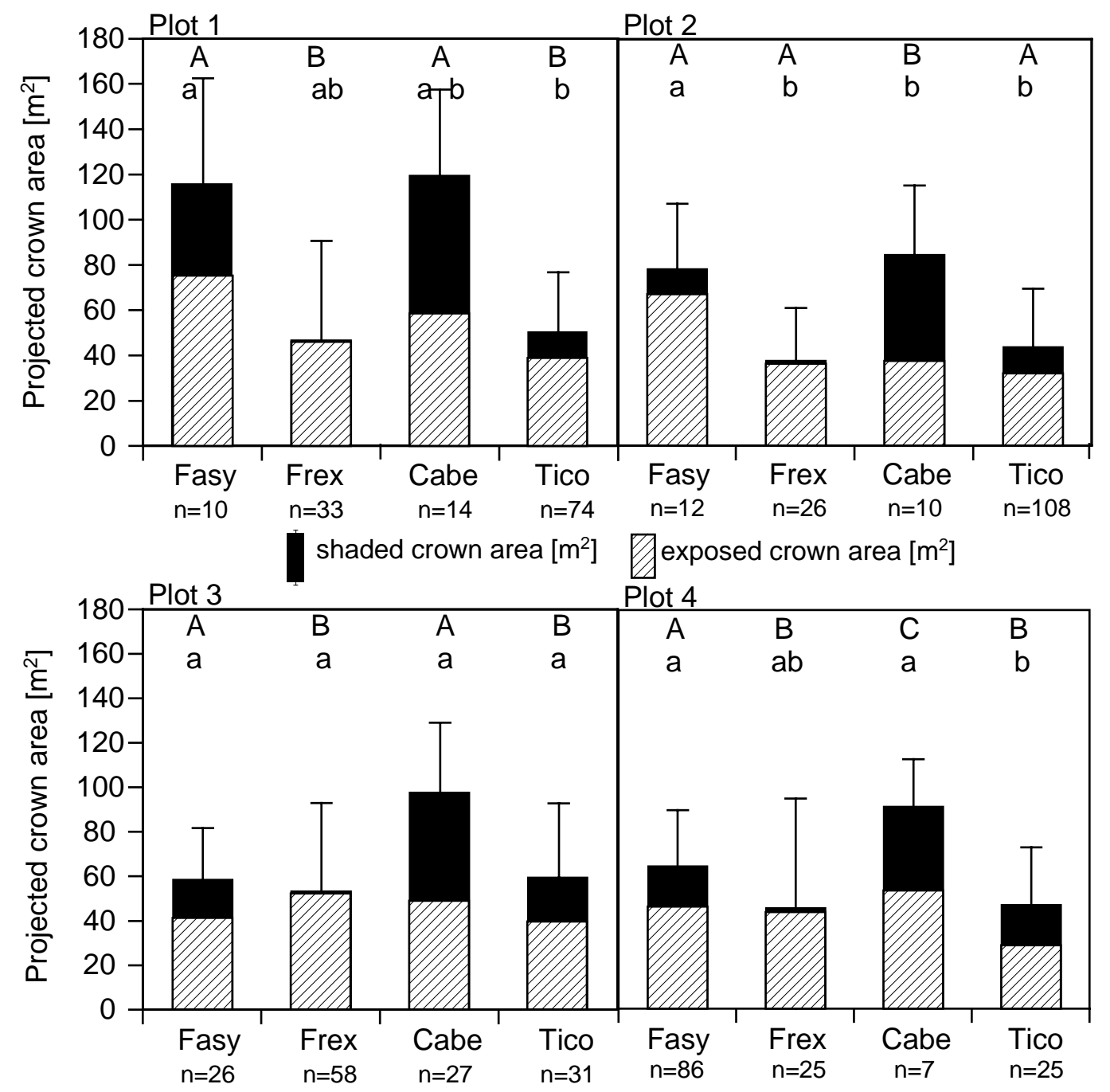

Abb. 4.7: Anteil der Sonnenkrone (schraffierter Bereich) an der gesamten Kronenschirmfläche für die Arten Rotbuche (Fasy), Esche (Frex), Hainbuche (Cabe) und Winterlinde (Tico) auf den vier Untersuchungsflächen. Die angegebene Standardabweichung bezieht sich auf die Werte der gesamten Kronenfläche. Unterschiedliche Großbuchstaben kennzeichnen signifikante Unterschiede zwischen den Gesamtkronenflächen der Arten einer Fläche. Unterschiedliche Kleinbuchstaben geben signifikante Unterschiede zwischen den Sonnenkronenanteilen an (Wilcoxon-U-Test mit BonferroniKorrektur, $\mathrm{p}<0.05$ ). 
Von allen Individuen dieser Arten, die in der obersten Kronenschicht vertreten waren, wurde die Kronenprojektionsfläche bestimmt. Zusätzlich zu den acht expositionsorientierten Radien wurde auch die Kronenausdehnung in Richtung unmittelbaren Nachbarbäume in einem Umkreis von elf Metern vermessen (s. Kap. 3.1.2). Vermessen wurde jeweils die gesamte Kronenausdehnung sowie der Anteil des nicht überschirmten Kronenbereichs.

Diese Differenzierung in Licht- und Schattenkrone erweist sich als wichtig im Artvergleich. Die untersuchten Hainbuchen haben im Mittel auf allen Flächen die größten Kronenflächen mit Ausdehnungen zwischen $84.33 \mathrm{~m}^{2}$ auf Fläche 2 bis zu $119.43 \mathrm{~m}^{2}$ auf Fläche 1 (Abb. 4.7). Auf den Flächen 2 und 4 weist sie signifikant größere Kronen auf als Buche, Esche und Linde. Der Größe der lichtexponierten Sonnenkrone unterscheidet sich jedoch nicht deutlich von den anderen Baumarten. Hier weisen die Rotbuchen auf Fläche 2 mit $75.22 \mathrm{~m}^{2}$ signifikant größere Flächen auf. Auf Fläche 3 liegen die Werte der Sonnenkronenflächen für alle Arten auf einem einheitlichen Niveau. Auf den drei anderen Flächen weist die Winterlinde mit Werten zwischen $28.71 \mathrm{~m}^{2}$ (Fläche 4) und $38.94 \mathrm{~m}^{2}$ (Fläche 1) signifikant kleinere Sonnenkronenanteile auf als die Rotbuche.

Auf Fläche 1 und 2 besitzt die Rotbuche kaum kleinere Kronenflächen als die Hainbuche. Ein wesentlicher Unterschied besteht zwischen diesen Baumarten jedoch in der Belaubungsdichte. Das Verhältnis der gesamten Blattfläche zur Kronenfläche einer Art kann als CAI (crown area index) ausgedrückt werden. Dieser beträgt für die Hainbuche 5.47 (s. Abb. 4.8), für die Buche jedoch nur 3.41. Damit ist der Anteil des Kronenraums, den die Hainbuche einnimmt, deutlich dichter belaubt.

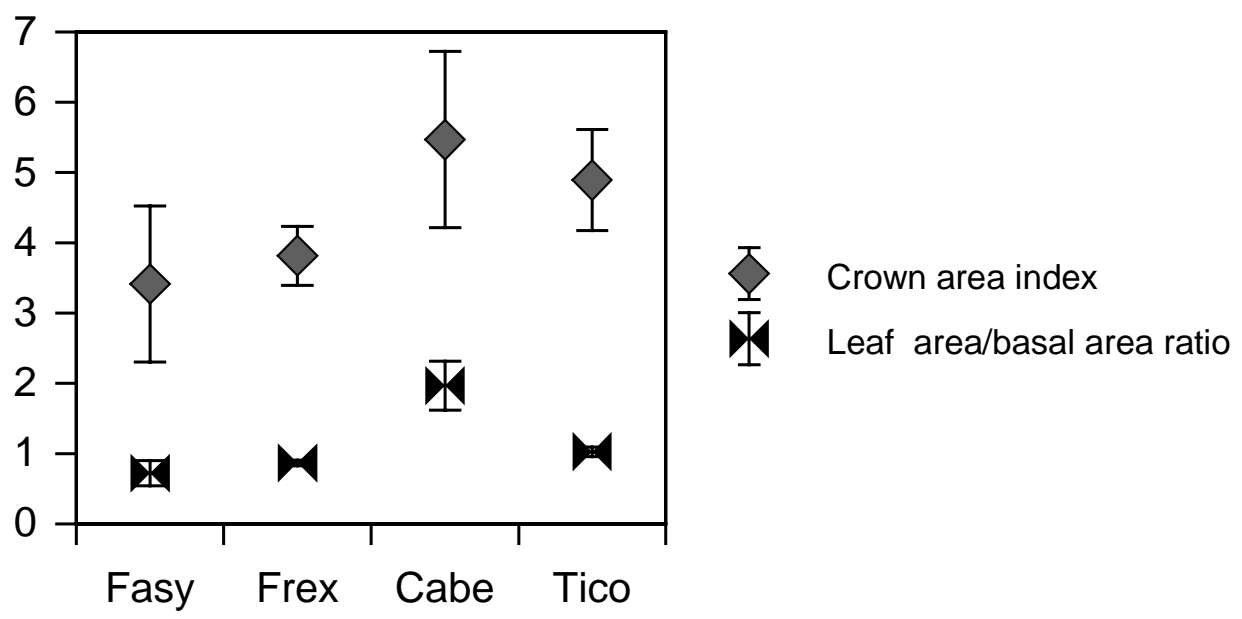

Abb. 4.8: Index-Werte zum Verhältnis Blattfläche/Kronenfläche (graue Rauten) und relative Blattfläche/Grundfläche (schwarze Dreiecke) für die vier untersuchten Baumarten. 
Analog dazu ist auch das Verhältnis der relativen Blattfläche zur relativen Grundfläche bei der Hainbuche mit dem Faktor 1.96 (s. Abb. 4.8, schwarze Dreiecke) deutlich höher als bei den anderen Arten, die mit Werten zwischen 0.72 für die Buche und 1.02 für die Winterlinde dicht beieinander liegen.
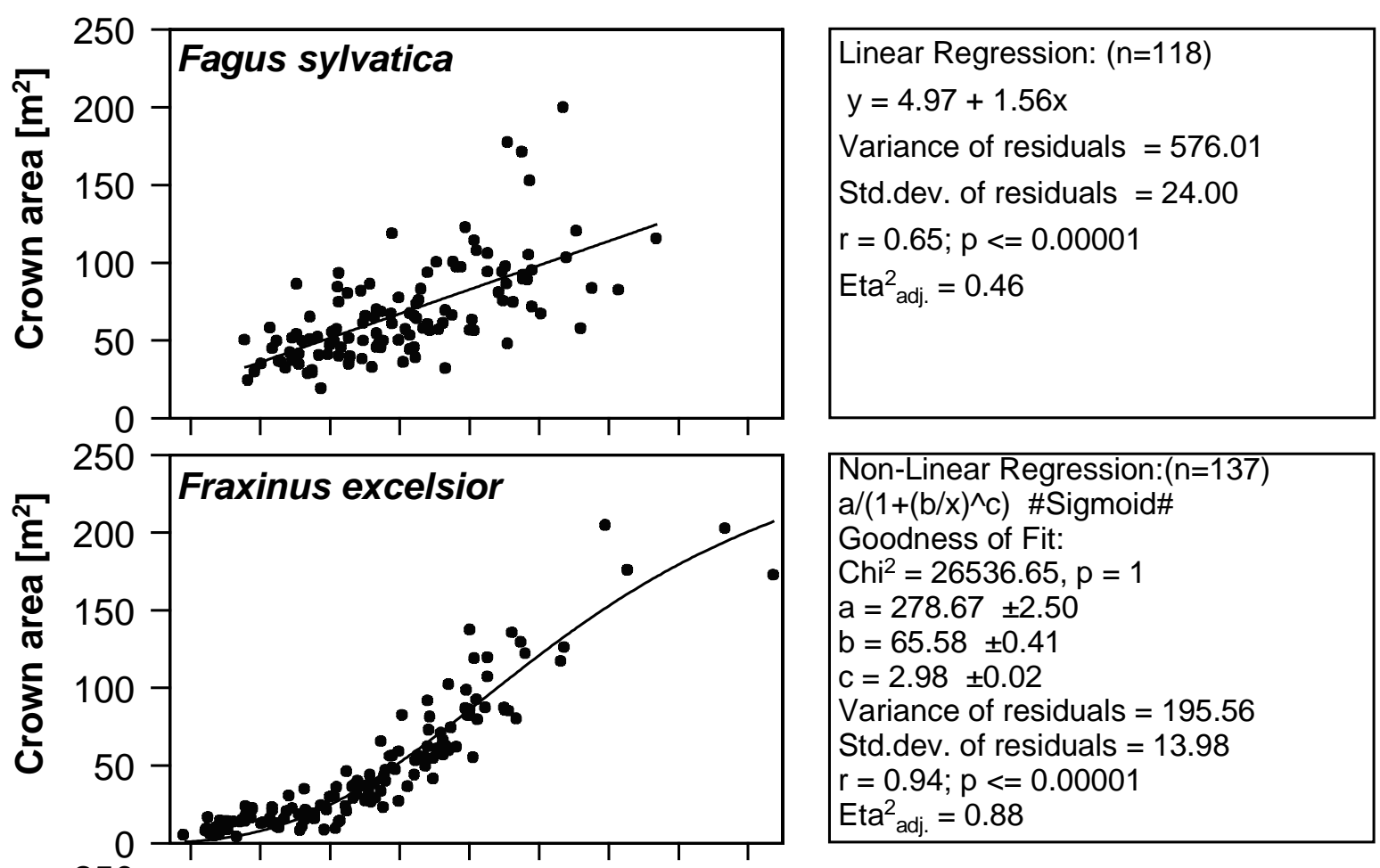

Non-Linear Regression:(n=137)

$\mathrm{a} /\left(1+(\mathrm{b} / \mathrm{x})^{\wedge} \mathrm{c}\right)$ \#Sigmoid\#

Goodness of Fit:

$\mathrm{Chi}^{2}=26536.65, \mathrm{p}=1$

$a=278.67 \pm 2.50$

$\mathrm{b}=65.58 \pm 0.41$

$c=2.98 \pm 0.02$

Variance of residuals $=195.56$

Std.dev. of residuals $=13.98$

$r=0.94 ; p<=0.00001$

$\mathrm{Eta}^{2}$ adj. $=0.88$

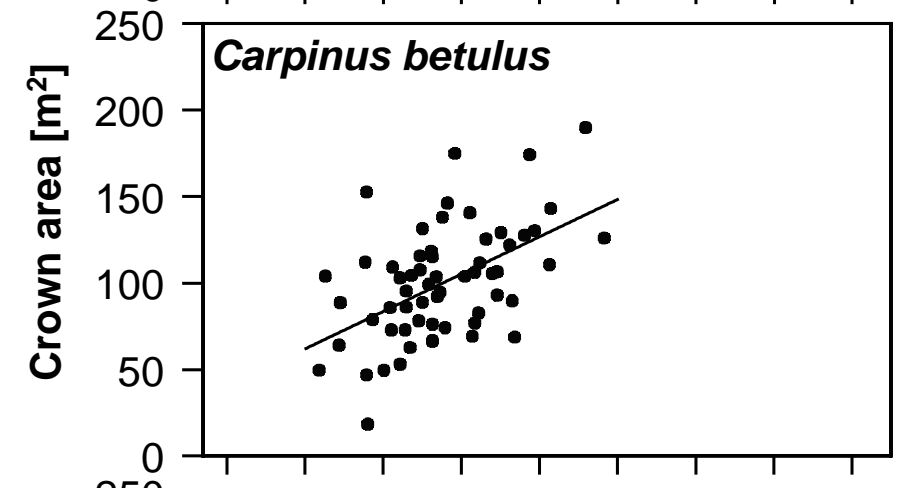

Linear Regression: $(\mathrm{n}=61)$

$y=18.88+2.16 x$

Variance of residuals $=819.45$

Std.dev. of residuals $=28.63$

$r=0.52 ; p=0.00005$

$\mathrm{Eta}^{2}{ }_{\text {adj. }}=0.2539$

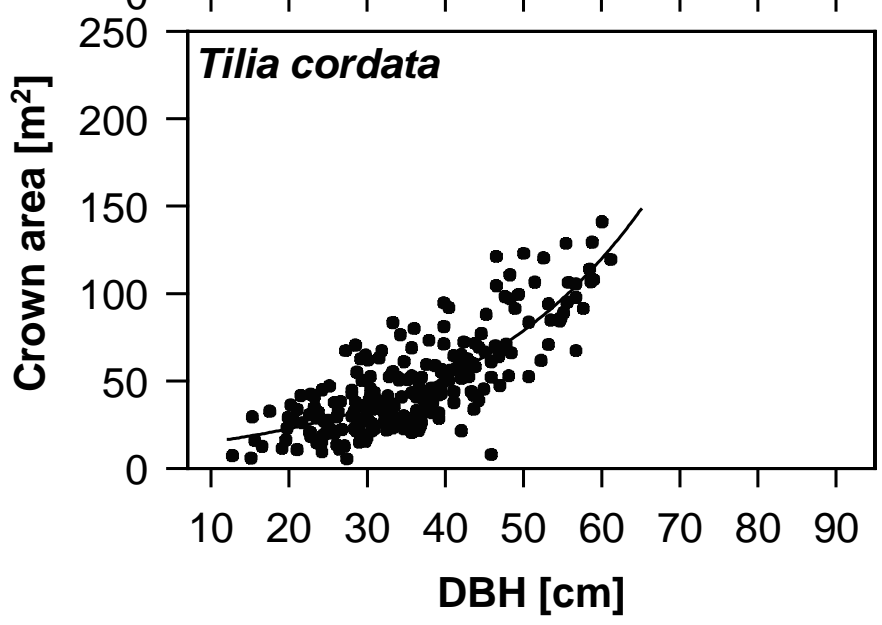

Non-Linear Regression:( $\mathrm{n}=234)$ $a+\exp \left(c^{*}(x-b)\right)$ \#Exp. Growth\# Goodness of Fit:

$\mathrm{Chi}^{2}=63908.11, \mathrm{p}=1$

$\mathrm{a}=0.78 \pm 0.62$

$\mathrm{b}=-52.90 \pm 1.38$

$c=0.04 \pm 0.0005$

Variance of residuals $=276.66$

Std.dev. of residuals $=16.63$

$r=0.81 ; p<=0.00001$

$\mathrm{Eta}^{2}$ adj. $=0.646$

Abb. 4.9: Artspezifische Beziehung zwischen Kronenfläche und Stammdurchmesser (DBH). 
Die weite horizontale Ausdehnung im Kronenraum erreicht die Hainbuche schon mit relativ geringem Stammumfang. Einzelne Individuen dieser Art weisen schon mit einem Brusthöhendurchmesser von $30 \mathrm{~cm}$ Kronenflächen von über $100 \mathrm{~m}^{2}$ auf. Dem entsprechend schwach ist die Zunahme der Stammstärke mit der Größe der Kronenfläche korreliert ( $\mathrm{r}=$ 0.52; s. Abb. 4.9).

Die Esche zeigt dagegen eine äußerst enge Beziehung zwischen Stammdurchmesser und Kronenfläche, die sich sehr gut, mit einem Korrelationskoeffizenten von 0.94, als SigmoidKurve beschreiben lässt. Kein Individuum dieser Art erreicht mit einem BHD von $30 \mathrm{~cm}$ eine Kronenfläche von $50 \mathrm{~m}^{2}$. Erst ab einem BHD von $40 \mathrm{~cm}$ breitet sich die Esche im Kronenraum verstärkt aus.

Dieser einheitliche Trend der kontinuierlichen horizontalen Ausdehnung mit dem Dickenzuwachs ist bei der Buche nicht zu erkennen. Hier streuen die Werte mit zunehmendem BHD immer stärker, so dass zwar eine lineare Beziehung zwischen beiden Parametern zu finden ist, diese aber mit einem r-Wert von 0.65 weniger stark ausgeprägt ist.

Die Winterlinde zeigt wiederum ein enges Verhältnis von Kronen- und Stammzuwachs. Die Streuung der Werte nimmt mit zunehmendem Stammdurchmesser ab und orientiert sich mit einem Korrelationskoeffizienten von 0.81 eng an einem exponentiellen Kurvenverlauf.

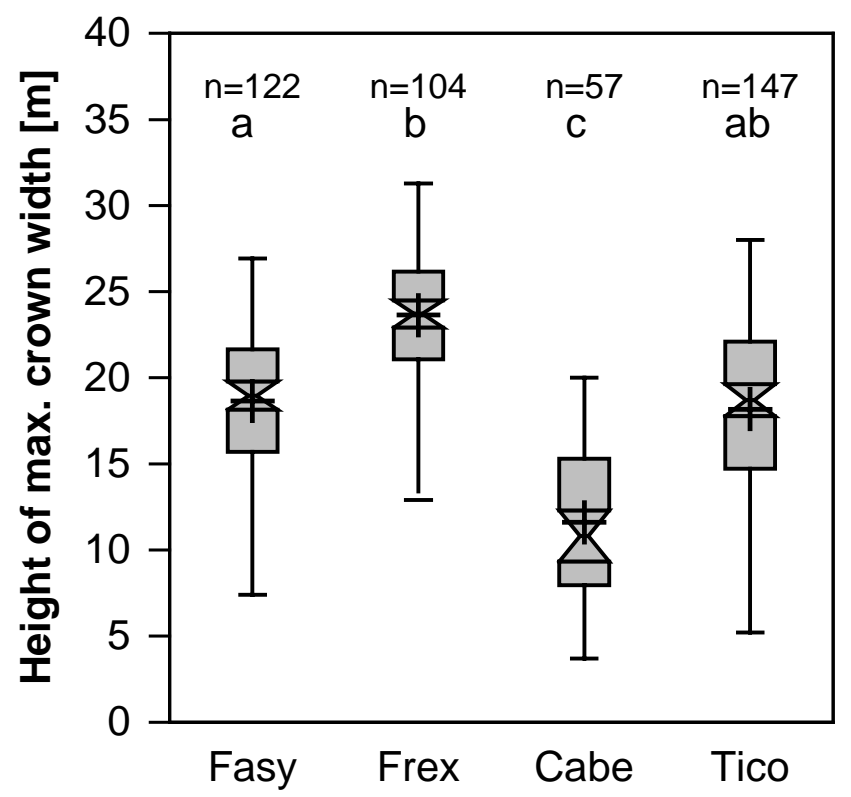

Abb. 4.10: Höhe der maximalen Kronenausdehnung der vier untersuchten Baumarten dargestellt in Box-Whisker-Plots. Die Einschnürung zwischen den grau dargestellten mittleren Quartilen zeigt den Median an, das schwarze Kreuz den Mittelwert. Unterschiedliche Kleinbuchstaben zeigen statistisch hochsignifikante Unterschiede zwischen den Arten an (Wilcoxon-U-Test, $\mathrm{p}<0.0001$ ). 
Neben den Unterschieden in der horizontalen Kronenausdehnung zeigen sich auch in der vertikalen Kronenstruktur deutliche Unterschiede zwischen den Arten. Buchen, Eschen und Hainbuchen erreichen ihre maximale Kronenausdehnung auf jeweils statistisch hochsignifikant unterschiedlichen Höhen im Bestand. Eschen erreichen ihre weiteste Ausladung im Mittel auf einer Höhe von $23.62 \mathrm{~m}$, in manchen Fällen sogar in der Wipfelregion über $30 \mathrm{~m}$. Buchen haben ihre maximale Kronenausdehnung auf einer Höhe von $18.64 \mathrm{~m}$ und damit rund fünf Meter tiefer als Eschen. Mit einer etwas geringeren Streuung der Werte liegt die maximale Kronenausdehnung der Hainbuchen bei durchschnittlich $11.60 \mathrm{~m}$ und damit nur knapp über dem Kronenansatz. Die Winterlinden weisen die breiteste Streuung der Werte auf. Der Mittelwert von $18.17 \mathrm{~m}$ liegt sehr nah bei dem der Buche, jedoch reichen die Extrema von $4 \mathrm{~m}$ bis $27 \mathrm{~m}$. Aufgrund dessen unterscheidet sich die Winterlinde in ihrem Höhenniveau der Kronenausdehnung nicht signifikant von der Esche.

\subsubsection{Nachbarschaftsinteraktion im Kronenraum}

Aus der unterschiedlichen Kronenstruktur der vier untersuchten Arten ergibt sich eine Vielfalt der Interaktionsformen und der Aufteilung des Kronenraums zwischen benachbarten Bäumen. In Abbildung 4.11 sind die Größen der Überlappungsflächen zwischen aneinander angrenzenden Kronen in Abhängigkeit von der Artzugehörigkeit der jeweiligen Nachbarbäume aufgetragen. In die Auswertung wurden nur Baumpaare einbezogen, die sich im Stammdurchmesser um nicht mehr als $15 \mathrm{~cm}$ voneinander unterschieden und eine Distanz zwischen den Stammfußpunkten von weniger als $11 \mathrm{~m}$ aufwiesen. Die Vielzahl der möglichen Artkombinationen lässt sich grundsätzlich einteilen in gleichartige und verschiedenartige Baumpaare. Bei den gleichartigen Paaren zeigen sich, analog zu der Höhenstratifizierung der maximalen Kronenbreite (s. Abb. 4.10), drei signifikant unterschiedliche Größen der Kronenüberlappung für Buchen, Eschen und Hainbuchen mit konspezifischem Nachbar (s. Abb. 4.11, die vier ersten Balken der Grafik zeigen die gleichartigen Baumpaare an; z.B. Fasy-Fasy für benachbarte Buchen). Benachbarte Eschen zeigen dabei mit durchschnittlich $3.73 \mathrm{~m}^{2}$ die geringsten Doppelüberschirmungen im Kronenraum. Auch Winterlinden zeigen in artgleichen Paaren kaum höhere Werte $\left(4.00 \mathrm{~m}^{2}\right)$. Bei benachbarten Hainbuchen sind es dagegen $15.35 \mathrm{~m}^{2}$. Die Buche nimmt mit $9.41 \mathrm{~m}^{2}$ Überlappungsfläche eine Mittelstellung ein. Zwischen diesen drei signifikant unterschiedlichen Größen-Niveaus der Überlappungsflächen zwischen gleichartigen Nachbarbäumen ist das Spektrum der Überlappungs-Werte der verschiedenartigen Nachbarn aufgespannt. Dabei weisen Eschen und Linden in Kombination 


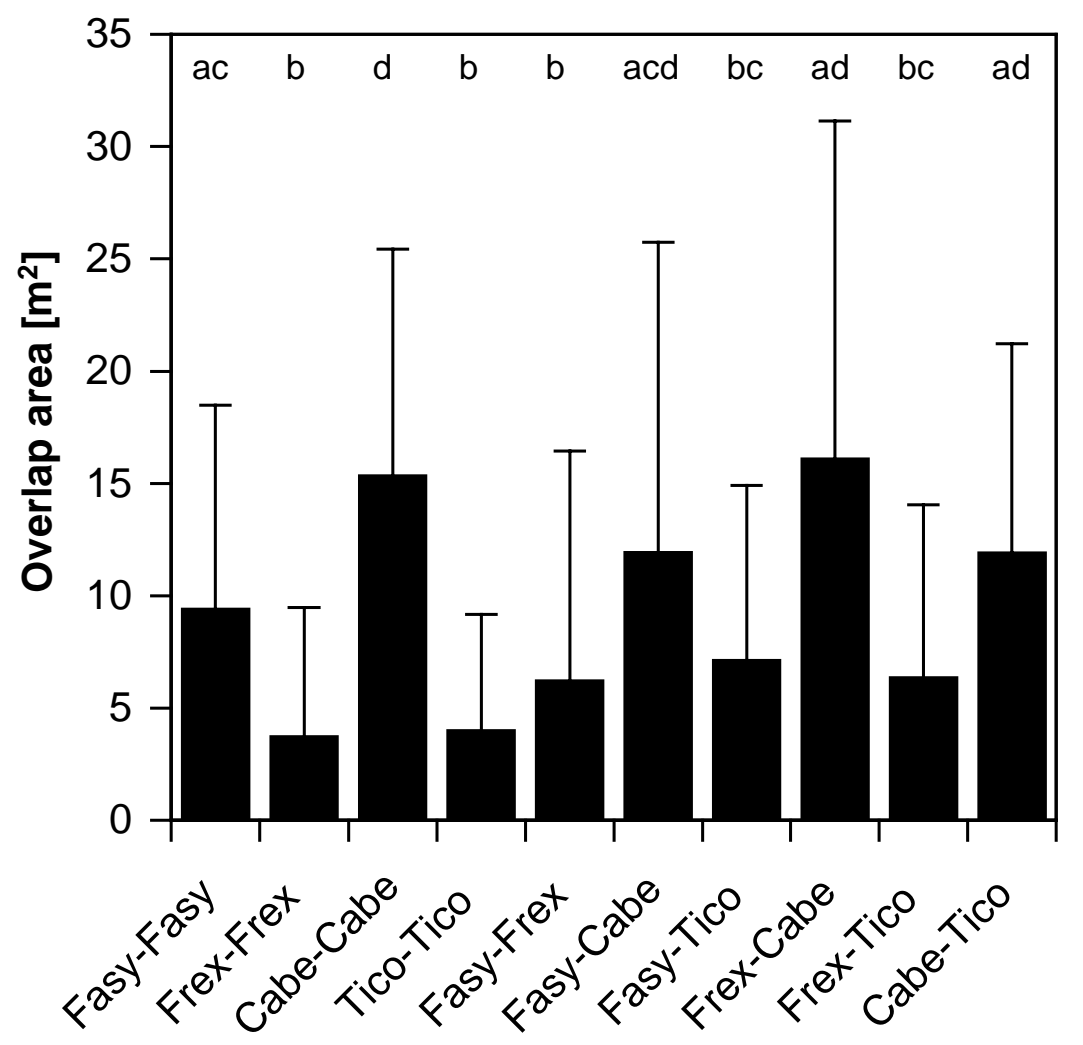

Abb. 4.11: Überlappungsflächen der Kronen benachbarter Bäume gleicher oder unterschiedlicher Artzugehörigkeit. Dargestellt sind die Mittelwerte und die Standardabweichung.Unterschiedliche Kleinbuchstaben geben signifikante Unterschiede zwischen den Artenkombinationen an (Wilcoxon-UTest; $\mathrm{p}<0.05)$.

mit anderen Arten stets höhere Überlappungen auf. Die größten Doppelüberschirmungen kommen mit durchschnittlich $16.10 \mathrm{~m}^{2}$ zwischen Eschen und Hainbuchen zustande. Auch in Nachbarschaft zu Buchen und Linden kommt es mit $11.93 \mathrm{~m}^{2}$ bzw. $11.92 \mathrm{~m}^{2}$ zu relativ großen Überlappungen.

Diese großen Überlappungen in Nachbarschaft zur Hainbuche nehmen $18.21 \%$ der Kronenfläche benachbarter Buchen ein. Damit ist der Anteil des geteilten Kronenraums nur leicht höher als zwischen benachbarten Buchen (16.26\%, s. Abb. 4.12). Noch größere Anteile der Kronenfläche in Nachbarschaft zur Hainbuche sind bei Winterlinden und Eschen doppelt überschirmt. Bei Linden sind es $22.00 \%$, bei Eschen $28.10 \%$. Bei letzteren kommt es in Einzelfällen zu einer Überlappung mit benachbarten Hainbuchen von bis zu $50 \%$; die Hainbuchen-Kronen reichen dabei also bis zur Mitte der Eschenkronen. Bei den jeweils benachbarten Hainbuchen nimmt der Überlappungsbereich mit 13.07\% (in Richtung benachbarter Linden) und $16.20 \%$ (in Richtung benachbarter Eschen) einen signifikant kleineren Anteil an der Kronenfläche ein. 
Für Eschen sind die Überlappungsbereiche in Nachbarschaft zu Hainbuchen damit fast drei Mal so groß wie in artreinen Kombinationen (10.04\%).

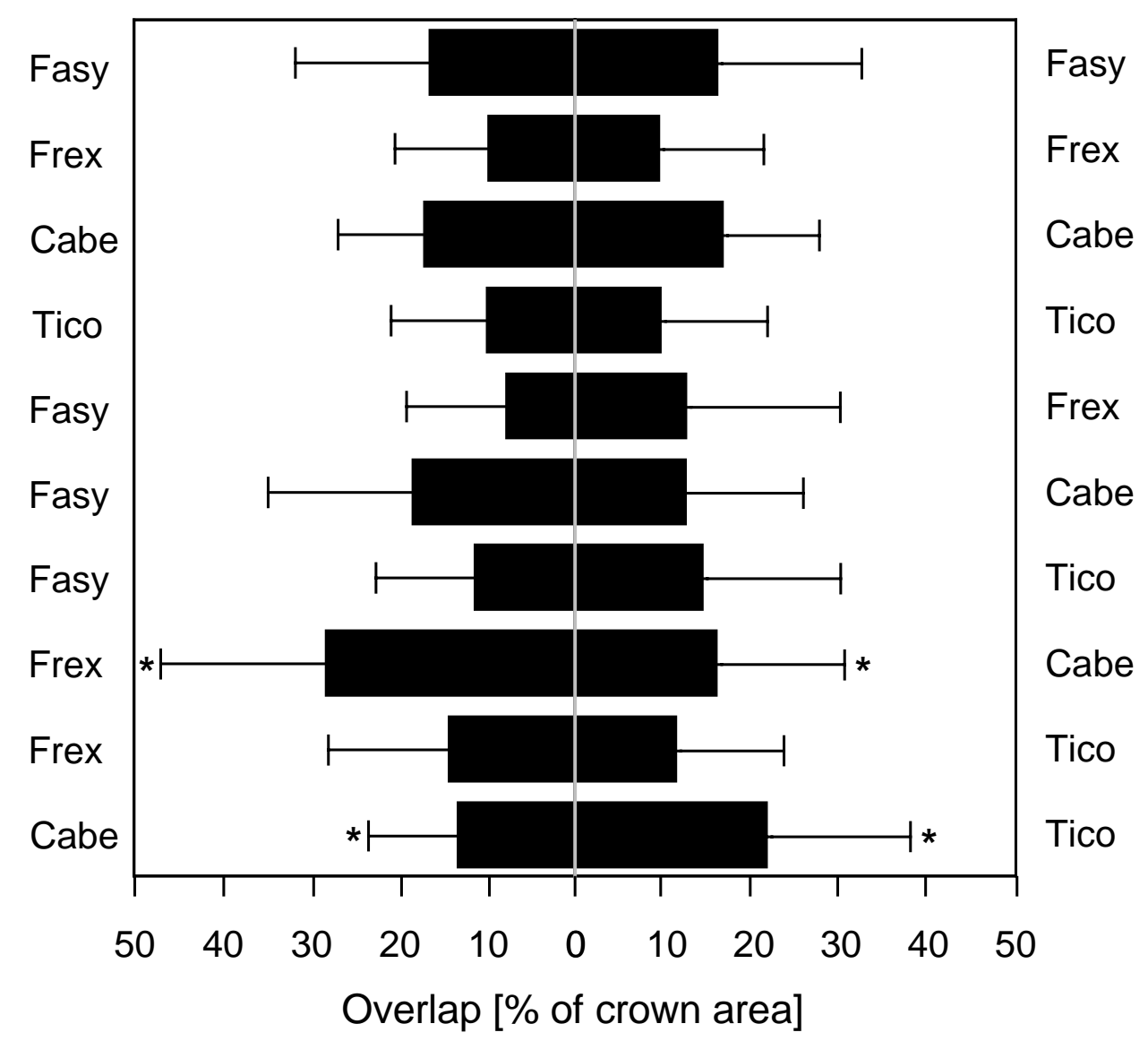

Abb. 4.12: Prozentualer Anteil der doppelt überschirmten Fläche zwischen zwei benachbarten Bäumen an der individuellen Kronenfläche. Bei den mit Sternchen gekennzeichneten Artpaaren bestehen signifikante Unterschiede im Verhältnis der Überlappungsfläche zur Gesamt-Kronenfläche (Wilcoxon-U-Test; $\mathrm{p}<0.05$ ).

Den geringsten Anteil von Überlappungsbereichen an der gesamten Kronenfläche weisen mit 7.54\% Buchen in Richtung benachbarter Eschen auf. Damit ist der Anteil an geteiltem Kronenraum im Vergleich zu artgleichen Nachbarn für diese Art weniger als halb so groß. 


\subsection{Mechanismen der Kronenraumeroberung}

\subsubsection{Astwachstum im seitlichen und oberen Kronenbereich}

Innerhalb von drei Vegetationsperioden wurden mit Hilfe der Hebebühne 682 Äste im oberen Kronenraum an insgesamt 47 Individuen vermessen. Dabei zeigt sich für das Trieblängenwachstum der vier untersuchten Baumarten ein recht einheitliches Bild: Das Längenwachstum ist im Wipfelbereich sehr viel größer als in den seitlichen Kronenbereichen und beträgt für alle Arten über $20 \mathrm{~cm}$ pro Jahr. Die Werte liegen zwischen $20.43 \mathrm{~cm}$ (Esche) und $22.67 \mathrm{~cm}$ (Buche) sehr dicht beieinander (Abb. 4.13).
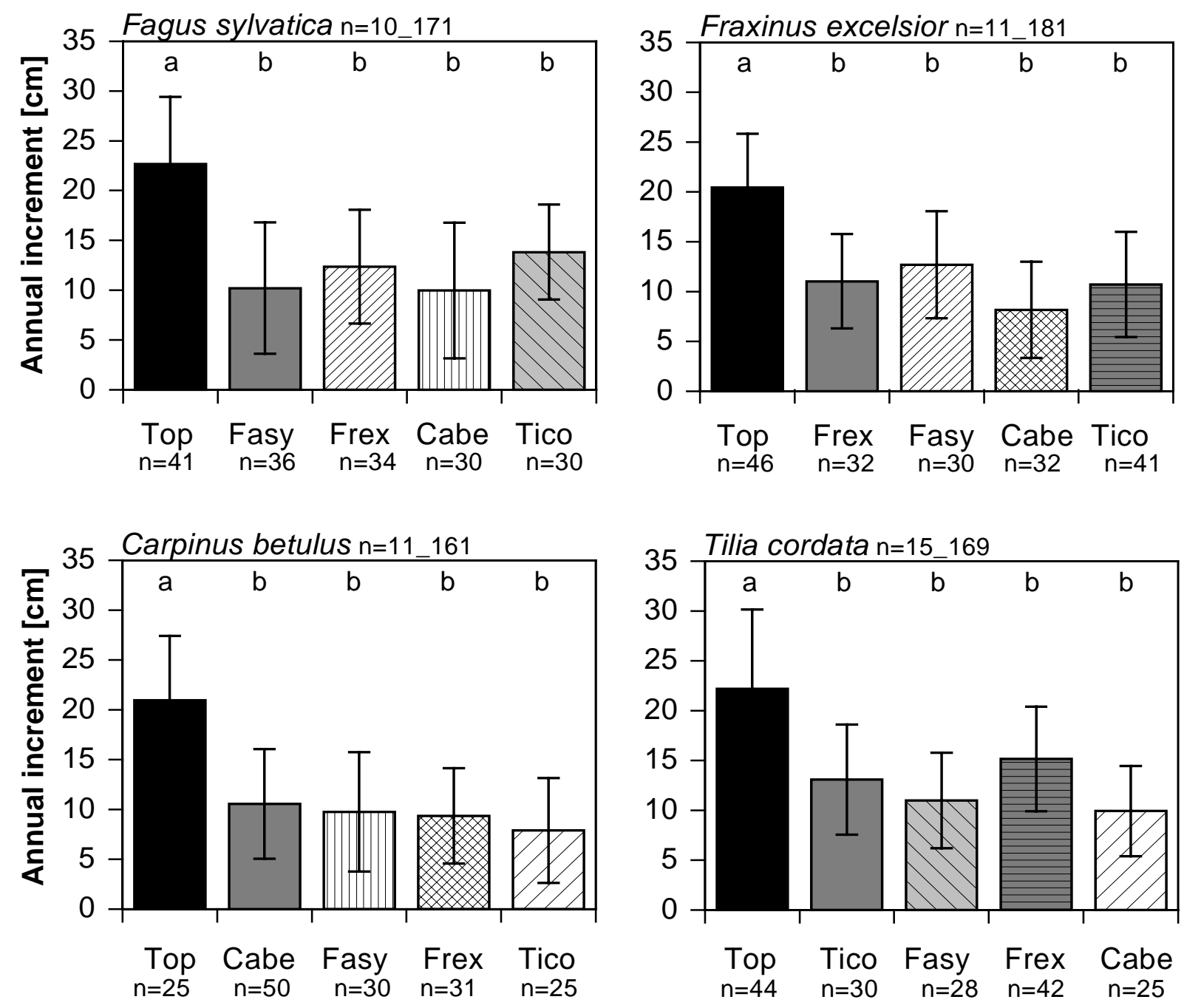

Abb. 4.13: Jährlicher Trieblängenzuwachs differenziert nach Wipfelbereich (schwarze Balken), intraspezifischen (graue Balken) und interspezifischen (gestreifte Balken) Kronennachbarschaften. Die n-Zahlen oberhalb der einzelnen Graphen geben die Zahl der untersuchten Individuen und nach dem Tiefstrich die Zahl der vermessenen Äste an. Unterschiedliche Kleinbuchstaben geben signifikante Unterschiede im Längenzuwachs zwischen verschiedenen Kronenregionen innerhalb einer Art an (Wilcoxon-U-Test; $\mathrm{p}<0.05$ ). 
Im seitlichen Kronenbereich sinkt das Längenwachstum um rund $50 \%$ auf Werte zwischen $9.4 \mathrm{~cm}$ bei der Hainbuche und 12.3 bei der Winterlinde. Die Hainbuchen zeigen nur sehr geringe Unterschiede zwischen den verschiedenen Nachbarschafts-Konstellationen, wobei das Längenwachstum der Zweige in Richtung gleichartiger Bäume mit $10.55 \mathrm{~cm}$ am höchsten ist. Bei den anderen drei Arten sind die Zuwachsunterschiede zwischen den einzelnen Nachbarschafts-Konstellationen etwas deutlicher ausgeprägt, jedoch nicht signifikant. Die Werte der intraspezifischen Kombinationen (dunkelgraue Balken) liegen mit Ausnahme der Hainbuche zwischen den interspezifischen. Grundsätzliche Unterschiede zeigen sich nicht.
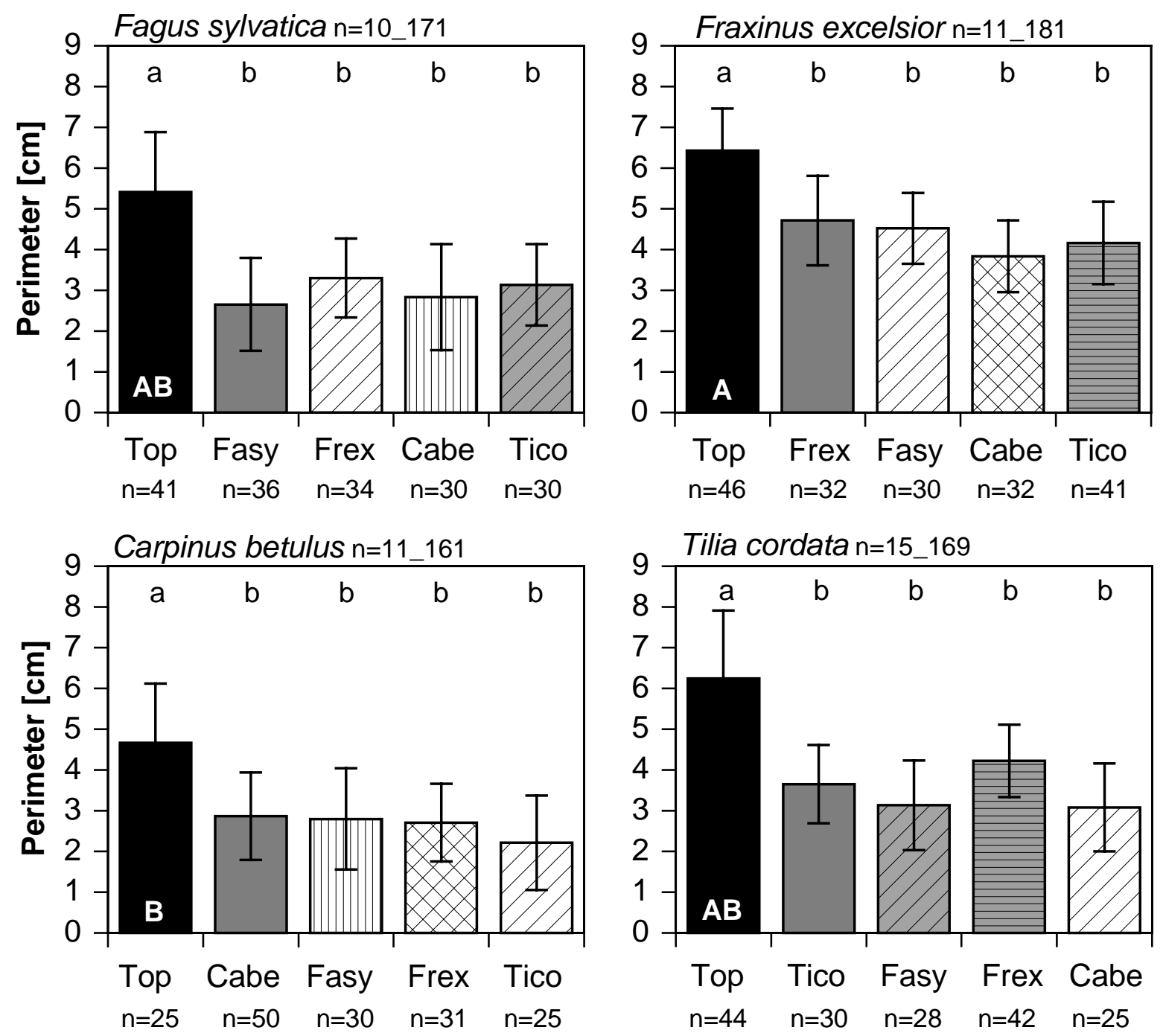

Abb. 4.14: Mittlerer Astumfang am Ansatz des sechsten Trieblängenabschnitts differenziert nach Wipfelregion und seitlichem Kronenbereich mit Nachbarschaftskontakt (weitere Erläuterungen s. Abb. 4.13). Unterschiedliche Kleinbuchstaben geben signifikante Unterschiede zwischen verschiedenen Kronenregionen innerhalb einer Art an, unterschiedliche Großbuchstaben zeigen signifikante Unterschiede in der Aststärke im Wipfelbereich zwischen den Arten (Wilcoxon-U-Test; $p<0.05$ ). 
Wie das Trieblängenwachstum ist auch das Dickenwachstum der untersuchten Äste im Wipfelbereich signifikant stärker ausgeprägt als im seitlichen Kronenraum (Abb. 4.14), wobei es ebenfalls keine Unterschiede zwischen den einzelnen Nachbarschafts-Konstellationen innerhalb einer Art gibt. Im Vergleich der Arten zeigt jedoch die Esche mit einem mittleren Astumfang von $6.43 \mathrm{~cm}$ im Wipfelbereich signifikant stärkere Äste als die Hainbuche mit $4.67 \mathrm{~cm}$.

Neben dem Längen- und Dickenwachstum eines Astes ist das Verzweigungsmuster einer Baumart ein wichtiges Merkmal für ihr Potenzial, Kronenraum zu erschließen und zu nutzen. Die untersuchten Eschen bilden pro Jahr durchschnittlich 0.7 Verzweigungen (Abb. 4.15) und somit signifikant weniger Seitenzweige als die anderen untersuchten Arten aus. Mit einem jährlichen Mittel von 0.47 bilden sie dementsprechend nur rund jedes zweite Jahr einen Langtrieb. Für Hainbuchen und Winterlinden liegt der Wert mehr als drei Mal so hoch bei 1.55, bzw. 1.44. Die untersuchten Buchen bilden durchschnittlich 1.3 Langtriebe pro Jahr. Allerdings bilden sie noch einmal genauso viele Kurztriebe $(<1 \mathrm{~cm}$ Länge) zusätzlich, was eine Besonderheit dieser Art darstellt.

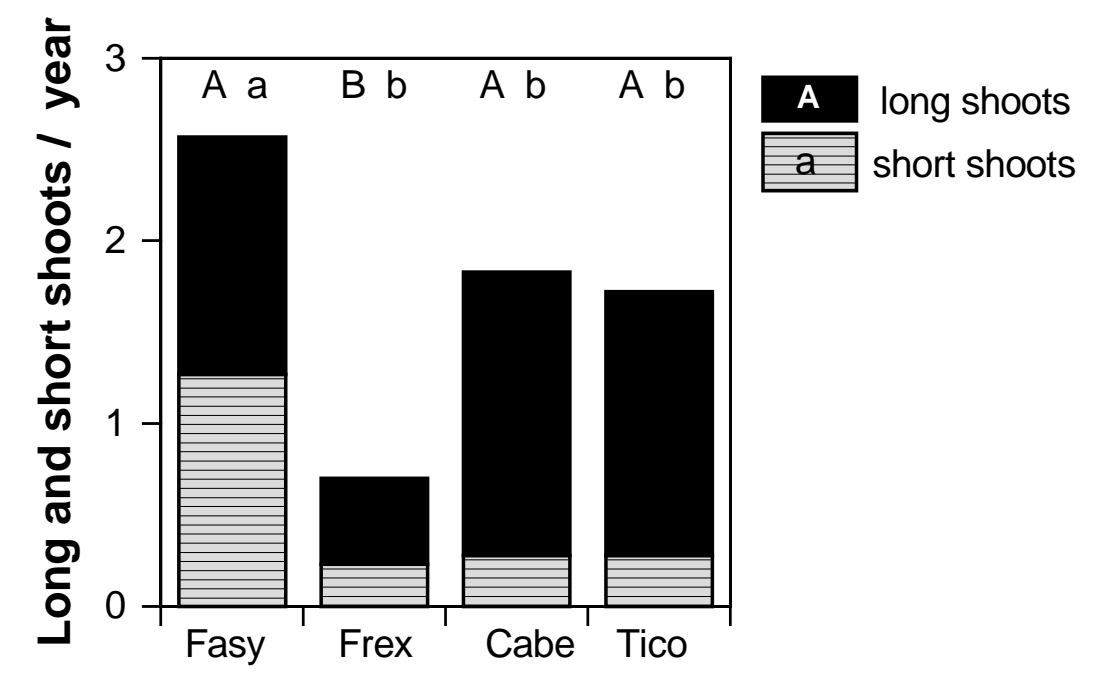

Abb. 4.15: Durchschnittliche Anzahl der jährlich ausgebildeten Langtriebe (schwarz) und Kurztriebe (quergestreift) pro Zweig für die vier untersuchten Baumarten. Unterschiedliche Großbuchstaben kennzeichnen signifikante Unterschiede zwischen den Arten für die Anzahl der Langtriebe pro Jahr, unterschiedliche Kleinbuchstaben zeigen signifikante Unterschiede in der Anzahl der Kurztriebe (Wilkoxon-U-Test; $\mathrm{p}<0.05$ ).

\subsubsection{Triebentwicklung und Lichtgenuss}

Aus der oberhalb der Triebspitzen der aufgenommenen Äste gemessenen PAR-Strahlung lässt sich aus dem Vergleich zur Freilandstrahlung der relative Lichtgenuss der einzelnen Triebe ermitteln. Das Längenwachstum des jüngsten Triebabschnitts wurde anschließend mit dem 
Lichtgenuss der Triebspitze korreliert. Dabei zeigt sich, dass zwischen beiden Parametern keine enge Beziehung besteht (s. Tab 4.4). Für Esche, Hainbuche und Winterlinde erklärt die relative Beleuchtungsstärke weniger als 50\% der Varianz. Lediglich bei der Buche gibt der Korrelationskoeffizient mit 0.72 eine etwas stärkere Abhängigkeit des Trieblängenwachstums vom Lichtgenuss an. Auch Anpassungsversuche mit nicht-linearen Beziehungen zeigten keine stärkere Abhängigkeit des Trieblängenwachstums vom relativen Lichtgenuss. Eine enge Beziehung besteht jedoch bei allen vier Baumarten zwischen der Trieblänge und der Anzahl der jährlich gebildeten Blätter und Knospen. Am schwächsten sind die Parameter Trieblänge und Knospenzahl bei der Esche mit $r=0.73$ korreliert. Der stärkste Zusammenhang besteht zwischen Längenwachstum und Anzahl der gebildeten Blätter $(r=0.84)$ und Knospen $(r=$ 0.88 ) bei der Hainbuche.

Tab. 4.4: Lineare Regression der Trieblänge auf die relative Beleuchtungsstärke an der Triebspitze (PAR), sowie die jährlich gebildete Blatt- und Knospenanzahl des jüngsten Triebabschnitts; $(\mathrm{p} \leq 0.001$, lineare Gleichung der Form $\mathrm{y}=\mathrm{a}+\mathrm{bx}$ ).

\begin{tabular}{|l|c|c|c|c|c|c|c|c|c|}
\hline \multicolumn{1}{|c|}{$\begin{array}{c}\text { Species } \\
(\mathrm{n}=158-181)\end{array}$} & \multicolumn{6}{|c|}{ shoot length/ PAR [\%] } & \multicolumn{2}{c|}{ shoot length / leaves } & \multicolumn{3}{|c|}{ shoot length/ buds } \\
\cline { 2 - 11 } & $\mathrm{r}$ & $\mathrm{a}$ & $\mathrm{b}$ & $\mathrm{r}$ & $\mathrm{a}$ & $\mathrm{b}$ & $\mathrm{r}$ & $\mathrm{a}$ & $\mathrm{b}$ \\
\hline F. sylvatica & 0.72 & 25.12 & 1.90 & 0.83 & 3.24 & 0.29 & 0.86 & 1.36 & 0.23 \\
\hline F. excelsior & 0.48 & 38.62 & 1.42 & 0.78 & 6.87 & 0.27 & 0.73 & 7.75 & 0.25 \\
\hline C. betulus & 0.45 & 26.15 & 1.97 & 0.80 & 3.01 & 0.32 & 0.86 & 2.60 & 0.32 \\
\hline T. cordata & 0.44 & 24.41 & 1.17 & 0.84 & 3.16 & 0.18 & 0.88 & 2.70 & 0.20 \\
\hline
\end{tabular}

Um ein etwas differenzierteres Bild vom Zusammenhang zwischen den erhobenen morphologischen Parametern Trieblänge, Blatt- und Knospenanzahl und der relativen Beleuchtungsstärke zu erhalten, wurden die untersuchten Zweige in Lichtklassen mit Intervallen von $20 \%$ eingeteilt (Abb. 4.16). Alle Baumarten zeigen dabei einen deutlichen Anstieg der Trieblängen (schräg schraffierte Balken) sowie der Blatt- und Knospenanzahl von der untersten zur höchsten Lichtklasse. Dieser Anstieg ist jedoch meist nicht kontinuierlich. So liegt das Längenwachstum der Buchenzweige mit einem relativen Lichtgenuss von 2040\% mit $5.81 \mathrm{~cm}$ über dem der Zweige in der nächst höheren Lichtklasse von 40-60\% (5.06). In den beiden obersten Lichtklassen steigt das Längenwachstum sprunghaft an, wohingegen die Anzahl der Knospen weit weniger stark ansteigt.

Bei den Eschen fällt auf, dass auch in den untersten Lichtklassen verhältnismäßig viele Blätter und Zweige ausgebildet werden. Eine drastische Erhöhung der Trieblängen-Werte ist erst in 
der obersten Lichtklasse festzustellen. Hier ist das Längenwachstum mit $17.41 \mathrm{~cm}$ um 55\% höher als in der Lichtklasse von 60-80\% $(7.83 \mathrm{~cm})$.

Das gilt auch für die untersuchten Hainbuchenzweige. Hier liegen die Trieblängen in den Lichtklassen von 0 bis $80 \%$ auf einem annähernd gleichen Niveau zwischen 5.16 und $7.46 \mathrm{~cm}$ und sind nur in der obersten Lichtklasse mit $17.71 \mathrm{~cm}$ deutlich erhöht.
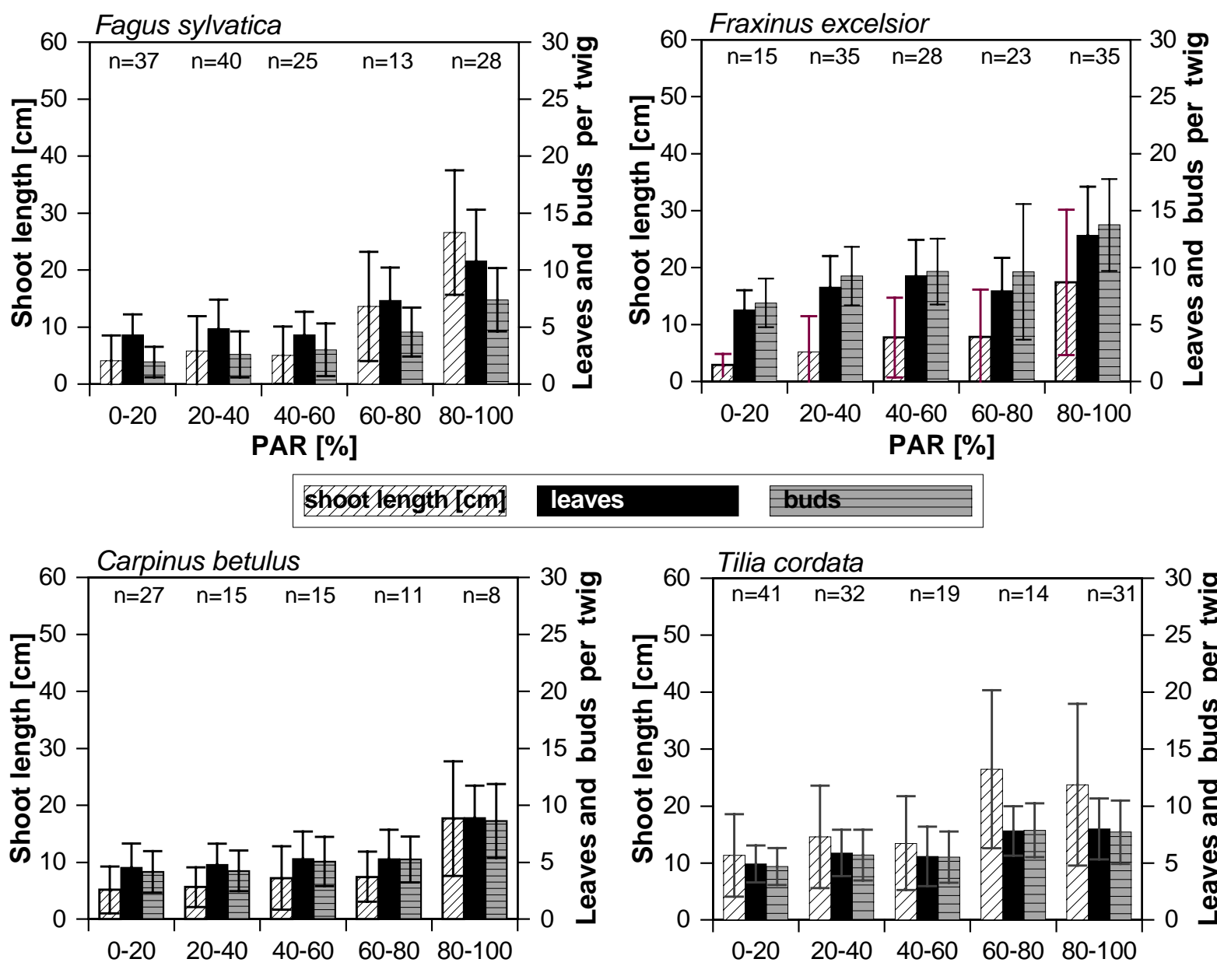

PAR [\%]

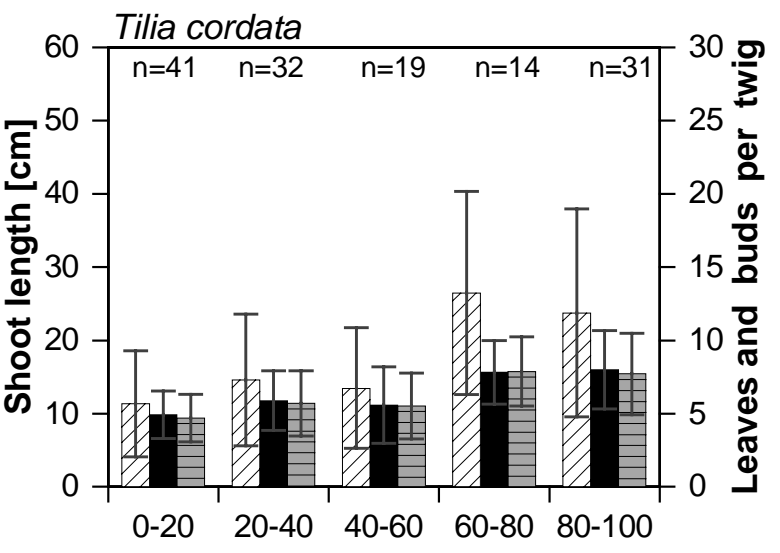

PAR [\%]

Abb. 4.16: Trieblängen sowie Blatt- und Knospenzahlen der untersuchten Äste von Buche, Esche, Hainbuche und Winterlinde eingeteilt nach dem relativen Lichtgenuss der jeweiligen Triebspitze

Die Winterlinde ist die einzige Baumart, deren Trieblängenwachstum in der obersten Lichtklasse nicht am größten ist. Es beträgt hier nur $23.73 \mathrm{~cm}$, wohingegen es bei den Zweigen mit einem relativen Lichtgenuss von $60-80 \%$ bei $26.46 \mathrm{~cm}$ liegt. Auch die mittlere Knospenzahl geht von 7.87 auf 7.71 leicht zurück. Nur die Anzahl der gebildeten Blätter steigt von 7.8 in der Lichtklasse von $60-80 \%$ auf 8.0 in der obersten Lichtklasse. 


\subsubsection{Triebentwicklung und mechanische Kroneninteraktion}

Während der Datenerhebung im Kronenraum zeigte sich, dass viele Äste eine durch Zweigabbrüche bewirkte Unterbrechung des ursprünglichen Wuchsmusters aufwiesen und stellenweise ein Seitenzweig die Funktion des Leittriebes übernommen hatte, wo die ursprüngliche Triebachse abgebrochen war. Diese offensichtlich auf Kollision mit den Ästen der Nachbarbäume bei stärkerem Wind zurückzuführenden Leittriebunterbrechungen wurden quantitativ erfasst und in ihrer relativen Häufigkeit ausgewertet. Dabei zeigt sich, dass im seitlichen Kronenraum bei allen Arten mehr als 50\% aller untersuchten Äste mindestens eine auf mechanische Schädigung durch Wind zurückführbare Unterbrechung der Haupttriebachse aufweist (Abb. 4.17).

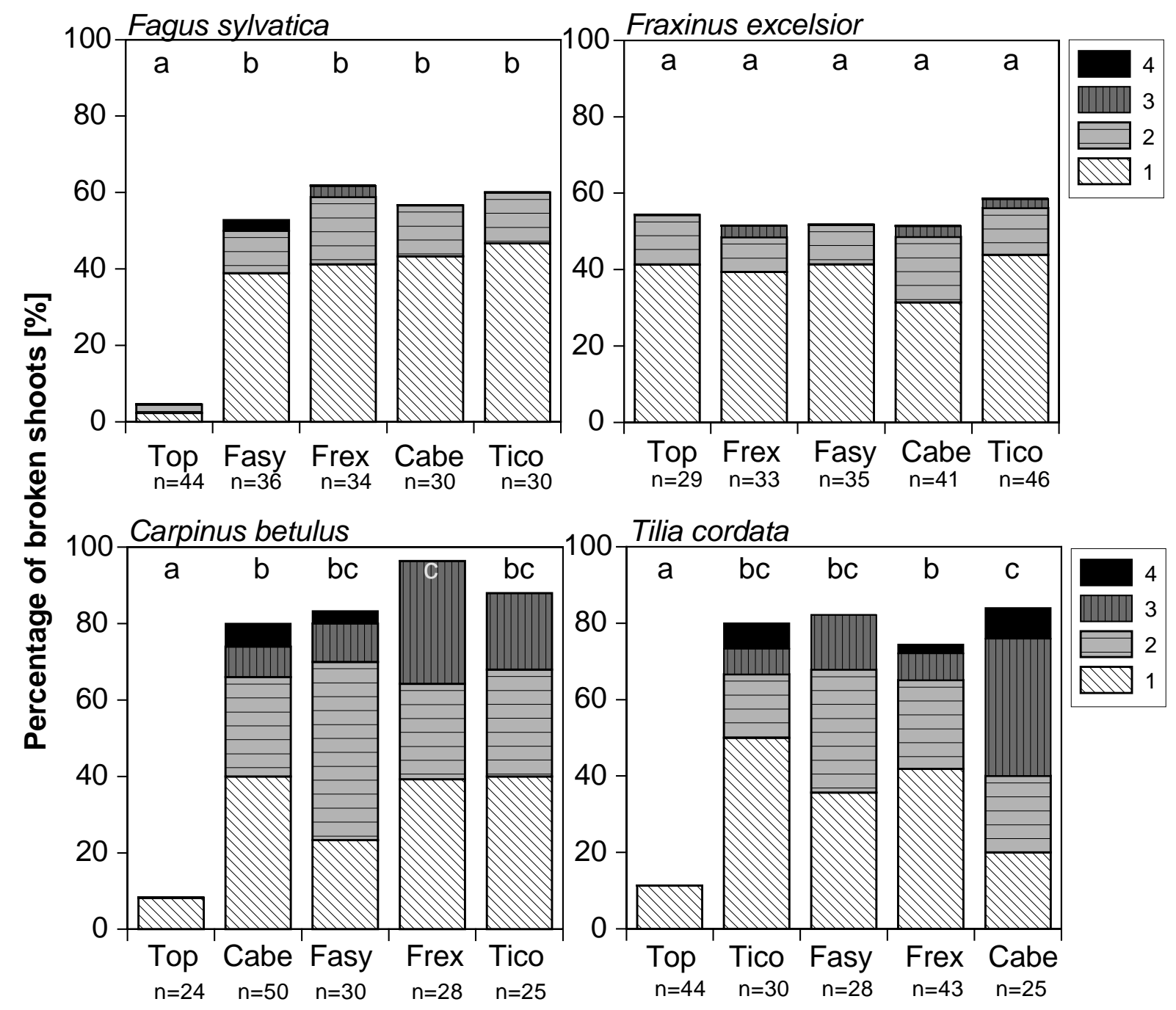

Abb. 4.17: Relative Häufigkeit von Leittriebunterbrechungen an den letzten sechs Jahresabschnitten der untersuchten Äste von Buche, Esche, Hainbuche und Winterlinde; die unterschiedlichen Schraffuren der Balkenabschnitte geben die Anzahl $(1-4)$ der Unterbrechungen pro Ast an. Unterschiedliche Kleinbuchstaben zeigen signifikante Unterschiede in der Abbruchrate zwischen den verschiedenen Nachbarschaftskonstellationen innerhalb einer Art (Wilkoxon-U-Test; $\mathrm{p} \leq 0.05$ ). 
Am stärksten betroffen ist die Hainbuche. Bei dieser Art ist die Triebachse innerhalb der erfassten sechs Jahre sehr häufig auch zweimal (in der Grafik quergestreifte Balkenabschnitte) oder auch dreimal (längsgestreifte Balkenabschnitte) unterbrochen. In Richtung benachbarter Eschen weisen 96\% aller untersuchten Hainbuchenzweige (siehe Grafik unten links: Carpinus betulus - Frex) mindestens eine Unterbrechung der Triebachse auf. Diese Rate liegt signifikant über den Abbruchraten in Richtung konspezifischer Nachbarbäume. Auch die der Hainbuche benachbarten Eschenzweige (Grafik oben rechts: Fraxinus excelsior - Cabe) zeigen verstärkt Mehrfachabbrüche im Vergleich zu den Eschenzweigen in Richtung anderer Nachbararten. Dennoch liegt der Gesamtprozentsatz von 51\% weit unter dem der betroffenen Hainbuchen und unterscheidet sich nicht signifikant von den Raten in anderen Nachbarschaftskonstellationen. Bei den untersuchten Eschenzweigen fällt weiterhin auf, dass die Abbruchrate im Wipfelbereich mit 54.34\% signifikant höher liegt als bei allen anderen Arten. Diese weisen Werte zwischen 4.54\% (Buche) und 11.36\% (Winterlinde) auf.

Bricht die Triebspitze aufgrund mechanischer Beschädigung ab, übernimmt ein Seitenzweig die Funktion der Haupttriebachse. So ist mit jedem Abbruch eine Änderung der Wuchsrichtung verbunden. Um zu erfassen, wie stark die häufigen Abbrüche im Kronenraum die Zweige von der Hauptwuchsrichtung abbringen und so die Kronenraumerschließung beeinträchtigen, wurde aus den gemessenen Trieblängen und den zusätzlich aufgenommenen Richtungs- und Steigungswinkeln pro Triebabschnitt ein Vektor für jeden Ast errechnet. Ins Verhältnis zur Summe der gemessenen Trieblängen gesetzt, lässt sich so die relative Astlänge bestimmen, die angibt wie weit der Ast tatsächlich in die Hauptwuchsrichtung vordringt.

Die Zweige im Wipfelbereich zeigten wenige Abbrüche; dem entsprechend waren wenige Änderungen in der Wuchsrichtung der einzelnen Treibabschnitte zu beobachten. So liegt die relative Astlänge hier für alle Arten nur knapp unter 100\% (s. Abb. 4.18). Bei Buchen- und Eschenzweigen zeigen sich im seitlichen Kronenbereich jedoch signifikante Differenzen zwischen Vektorlänge und aufsummierter Trieblänge. Die Mittelwerte liegen bei der Buche zwischen 93.65\% (in Richtung benachbarter Hainbuchen) und 95.65\% (im Kontakt mit gleichartigen Nachbarn). Bei der Esche liegen die Werte ebenfalls dicht zusammen zwischen 94.71\% (Nachbar: Hainbuche) und 96.22\% bei konspezifischen Nachbarn.

Aufgrund der sehr großen Streuung der Werte, zeigen sich bei der Hainbuche keine signifikanten Unterschiede zwischen den verschiedenen Kronenbereichen. Hier kommt es im Mittel in Nachbarschaft zu Buchen zu einer Längeneinbuße von fast 10\%. Der Minimalwert liegt bei $62.3 \%$. Wie die Hainbuchen zeigen auch die Winterlinden die geringste Einbuße der 
relativen Astlänge in Nachbarschaft zu Eschen. Hier gleicht die Geradlinigkeit des Wuchsverlaufes dem des Wipfelbereichs (97\%). Auch die stärkste Reduktion der relativen Astlänge zeigt die Linde im Kontakt zu benachbarten Buchenkronen. Hier wird der Mittelwert (schwarzes Kreuz) durch einige Zweige mit extremen Richtungsänderungen auf $86.44 \%$ herabgesenkt. Der Minimalwert liegt außerhalb der Skalierung bei 45.99\%.

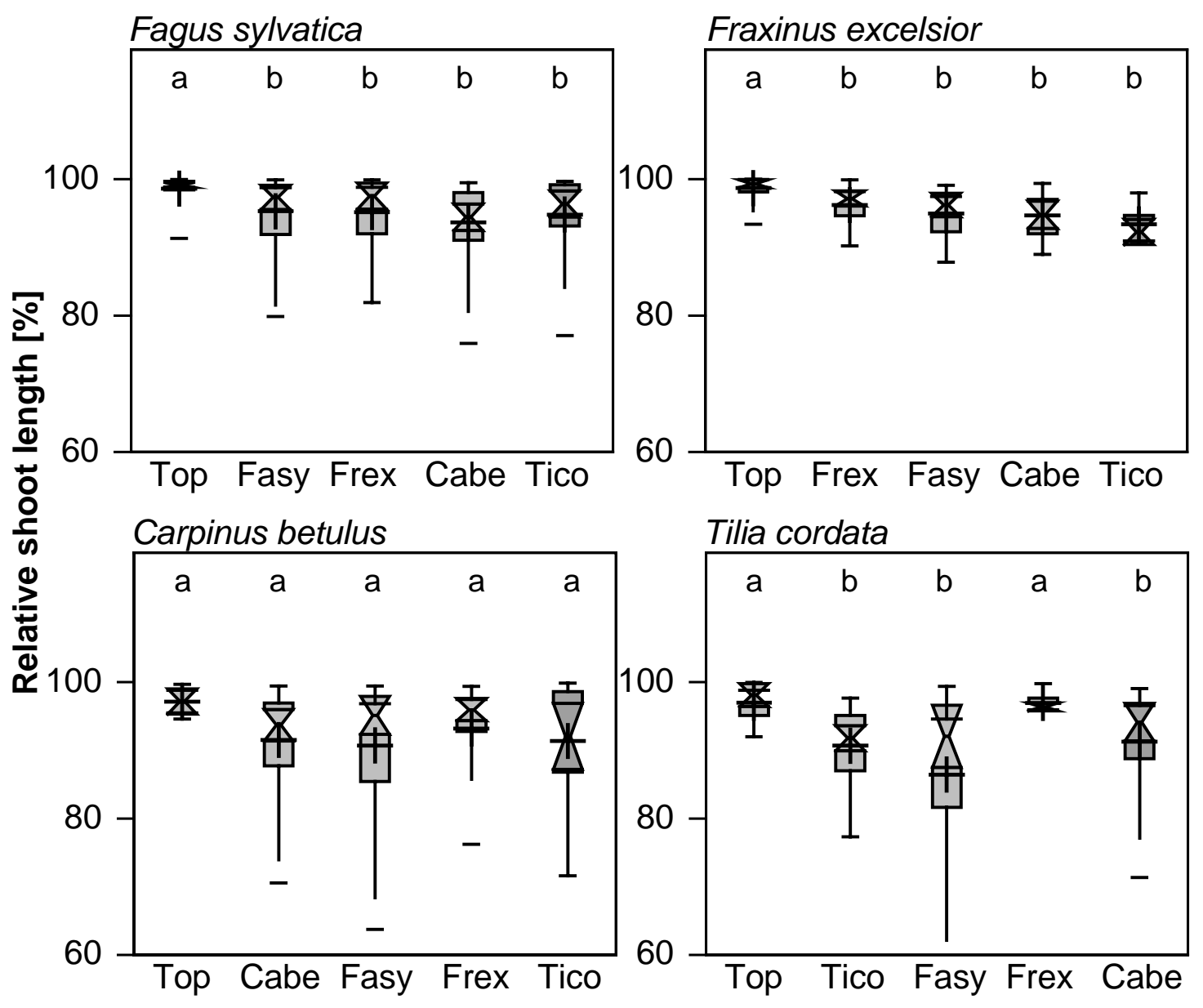

Abb. 4.18: Prozentualer Anteil der Vektorlänge der Triebe in die Hauptwuchsrichtung im Verhältnis zur Summe der gemessenen Trieblängen pro Ast. Unterschiedliche Kleinbuchstaben zeigen signifikante Unterschiede zwischen den verschiedenen Nachbarschaftskonstellationen innerhalb einer Art (Wilkoxon-U-Test; $\mathrm{p} \leq 0.05$ ).

\subsubsection{Räumliche Interaktion - die Kontaktzone}

Die vom obersten Punkt im Kronenraum ausgelotete vertikale Ausdehnung der gemeinsamen beblätterten Kontaktzone zwischen zwei Nachbarbäumen ist je nach Artenkombination sehr unterschiedlich. Die kürzesten gemeinsamen Kontaktzonen weisen mit $1.91 \mathrm{~m}$ benachbarte Eschen auf (Abb. 4.19: Frex-Frex). Der Bereich, in dem beblätterte Zweige dieser Art aneinander grenzen, beschränkt sich auf einen Höhenabschnitt von 23.9 bis 25.8 m, obwohl 
der Kronenansatz der Esche schon bei $16.2 \mathrm{~m}$ liegt. Damit besteht ein direkter Kronenkontakt zwischen Eschen nur auf 15.4\% der gesamten Kronenlänge. Auch in der Nachbarschaft zu anderen Baumarten bilden Eschen nur sehr kurze, wenige Meter unter dem Wipfel gelegene Kontakt-Zonen. Die längste Kontakt-Zone mit benachbarten Eschen bilden Winterlinden mit 3.04 m Länge.

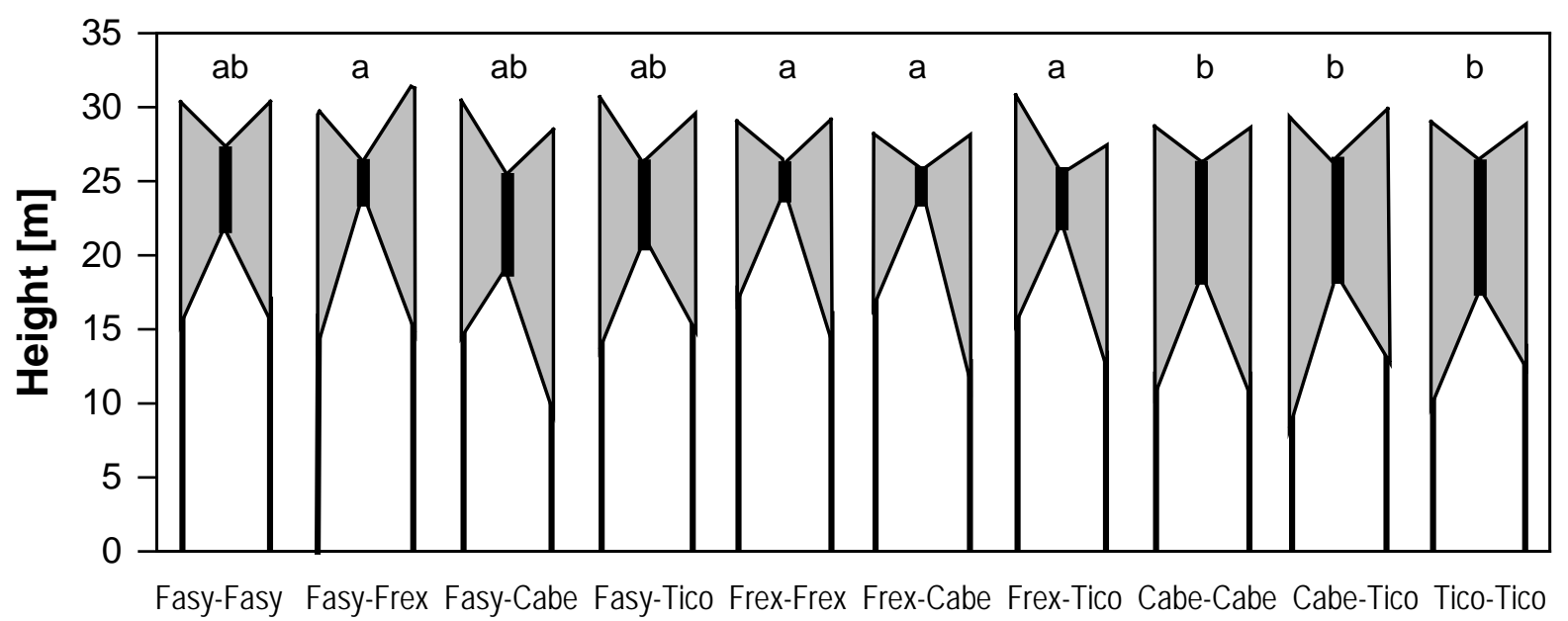

Abb. 4.19: Vertikale Ausdehnung der gemeinsamen beblätterten Kontaktzone (dicke schwarze Linien) zwischen den untersuchten Baumarten in den zehn verschieden Nachbarschafts-Konstellationen. Grau dargestellt sind die Kronenansatz- und Wipfelhöhen der jeweils untersuchten Individuen

Die Linde weist insgesamt die längsten Kontaktzonen auf, die in Nachbarschaft zu anderen Arten noch deutlich länger sind. So liegen die Kontakt-Zonen zu benachbarten Buchen bei 5.27 m, zu Hainbuchen bei 7.34 m. Die längsten Kontaktzonen haben die untersuchten Winterlinden jedoch in intraspezifischen Nachbarschafts-Konstellationen. Diese sind im Durchschnitt $8.35 \mathrm{~m}$ lang und erstrecken sich über 52.6\% der gesamten Kronenlänge. Auch Hainbuchen bilden, mit Ausnahme zu benachbarten Eschen, lange Kontakt-Zonen aus. Diese sind ebenfalls in intraspezifischen Kombinationen am längsten $(7.71 \mathrm{~m})$ und erstrecken sich über ein Höhenniveau von 18.2 bis 25.9 m. Der Kronenansatz dieser Art liegt mit $10.1 \mathrm{~m}$ noch deutlich darunter, sodass die gemeinsame Kontakt-Zone auch bei dieser Art nicht mehr als $42.6 \%$ der gesamten Kronenlänge einnimmt.

Zusätzlich zur Länge der gemeinsamen beblätterten Kontaktzone wurde auch aufgenommen, wie weit die Kronen horizontal ineinander greifen. Die Intensität des Kronenkontaktes wurde in drei Klassen eingeteilt (von Klasse $1=$ Kronenscheu, über Klasse 2 = Ineinandergreifen bis $50 \mathrm{~cm}$, zu Klasse 3 = Ineinandergreifen über $50 \mathrm{~cm}$ ). In der Intensität des horizontalen 
Kronenkontaktes teilen sich die zehn möglichen Nachbarschaftskonstellationen in zwei deutlich voneinander getrennte Gruppen: Auf der einen Seite zeigen benachbarte Eschen und benachbarte Hainbuchen jeweils in artgleichen Paaren klar das Phänomen der Kronenscheu (s. Tab. 4.5). Eschen und Hainbuchen in direkter Nachbarschaft sowie Eschen und Buchen zeigen ebenfalls verstärkt Kronenscheu (mittlerer Klassenwert 1.5). Jedoch ist hier der Unterschied zu anderen Konstellationen nicht mehr signifikant.

Das andere Extrem zeigt die Esche in Nachbarschaft zur Winterlinde. In dieser Konstellation kommt es fast immer zu einem Ineinandergreifen der Kronen von über $50 \mathrm{~cm}$ (2.8). Eine Tendenz in diese Richtung zeigen mit Klassen-Mittelwerten zwischen 2.4 und 2.6 auch alle anderen Art-Kombinationen mit Winterlinde.

Tab. 4.5: Klassifizierung der horizontalen Kroneninteraktion (Klassen von 1-3 s. Text) mit Standardabweichung (sd) und signifikanten Unterschieden zwischen den verschiedenen Nachbarschaftskonstellationen (sig.); (Wilkoxon-U-Test; $\mathrm{p} \leq 0.05$ ).

\begin{tabular}{lccc}
\hline Neighbours & $\begin{array}{c}\text { Class of crown } \\
\text { interference }\end{array}$ & sd & sig. \\
\hline Fasy-Fasy & 2.3 & 0.48 & $\mathrm{ab}$ \\
Fasy-Frex & 1.5 & 0.53 & $\mathrm{bc}$ \\
Fasy-Cabe & 2.33 & 0.71 & $\mathrm{ab}$ \\
Fasy-Tico & 2.4 & 0.52 & $\mathrm{ab}$ \\
Frex-Frex & 1.2 & 0.42 & $\mathrm{c}$ \\
Frex-Cabe & 1.5 & 0.53 & $\mathrm{bc}$ \\
Frex-Tico & 2.8 & 0.42 & $\mathrm{a}$ \\
Cabe-Cabe & 1.13 & 0.35 & $\mathrm{c}$ \\
Cabe-Tico & 2.6 & 0.7 & $\mathrm{ab}$ \\
Tico-Tico & 2.4 & 0.52 & $\mathrm{ab}$ \\
\hline
\end{tabular}




\subsection{Dendroökologische Untersuchungen}

\subsubsection{Stammzuwachs und Trieblängenwachstum}

An jeweils sechs Individuen pro Art (fünf für die Winterlinde), an denen Messungen zum Trieblängenwachstum durchgeführt wurden, wurde auch das Dickenwachstum des Stammes untersucht. Für diese Bäume lässt sich das Wachstum der einzelnen Kronenkompartimente (Wipfelzweige und seitliche Kronenbereiche) direkt mit dem Stammzuwachs der Jahre 1997 bis 2004 vergleichen (Abb. 4.20).

Die untersuchten Buchen weisen in dieser Periode Basalflächenzuwächse von 14.51 (1998) bis $24.36 \mathrm{~cm}^{2}$ im Jahr 2001 auf. Im Wachstum der Wipfelzweige, das im Mittel $21.75 \mathrm{~cm}$ beträgt, liegt der Spitzenwert im Jahr 2003 (27.11 cm) und fällt zum Jahr 2004 drastisch ab auf $12.28 \mathrm{~cm}$. Auch die seitlichen Kronenbereiche zeigen einen starken Zuwachsrückgang im Jahr 2004. Für das über alle Kronenbereiche gemittelte Längenwachstum der Buchenzweige zeigt sich ein signifikanter, negativer Wachstumstrend für den Untersuchungszeitraum (Tab. 4.6; rechte Spalte). Durch die starken Schwankungen im Stammzuwachs zwischen den Jahren 1998 und 2001 ist dieser Trend für den Basalflächenzuwachs nicht signifikant, ein Abnehmen der Zuwächse ab 2001 ist dennoch deutlich sichtbar (Abb. 4.20).

Tab. 4.6: Artspezifischer Wachstumstrend des Basalflächenzuwachses und des Trieblängenzuwachses für die Jahre 1997 bis 2004 (lineare Gleichung der Form y=a+bx).

\begin{tabular}{lcc}
\hline & \multicolumn{2}{c}{$\begin{array}{c}\text { Growth trend 1997-2004 } \\
\text { Linear regression }(\mathrm{p}<0.05)\end{array}$} \\
\hline Species & Basal area & Shoot growth \\
\hline Fagus sylvatica & - & $\mathrm{r}=-0.72$ \\
Fraxinus excelsior & - & $\mathrm{r}=-0.72$ \\
Carpinus betulus & - & $\mathrm{r}=-0.93$ \\
Tilia cordata & - & - \\
\hline
\end{tabular}

Das Trieblängenwachstum der Esche weist mit $12.27 \mathrm{~cm}$ im Mittel etwas niedrigere Werte auf als das der Buche (13.12). Die Stammzuwächse sind deutlich größer als die der Buchen. Diese liegen bei $32.81 \mathrm{~cm}^{2}$, der Spitzenwert liegt bei $44.86 \mathrm{~cm}^{2}$ im Jahr 1999. Das Trieblängenwachstum nimmt wie bei der Buche zum Jahr 2004 hin signifikant ab (s. Tab. 4.6), der Stammzuwachs zeigt gleichzeitig keinen negativen Trend. Im Gegensatz zu allen anderen untersuchten Baumarten zeigt die Esche keinen Zuwachsrückgang im Jahr 2004. 


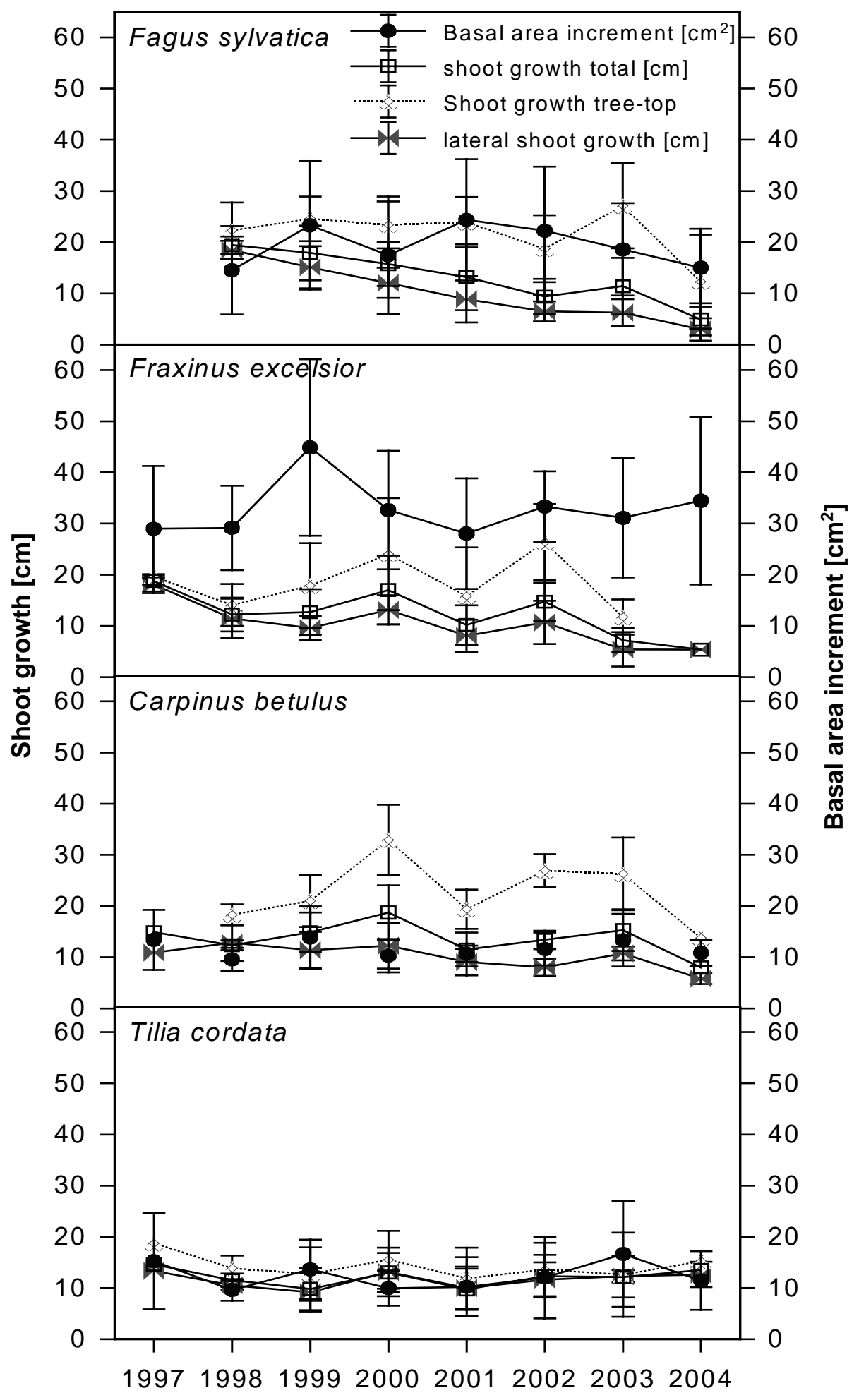

Abb. 4.20: Vergleich des individuellen Basalflächenzuwaches mit dem Trieblängenwachstum differenziert nach Wipfelbereich (shoot growth tree-top) und seitlichen Kronenbereichen (lateral shoot growth). Dargestellt sind die Mittelwerte aus jeweils sechs Individuen pro Art für Buche, Esche und Hainbuche und fünf Individuen der Winterlinde.

Das Zweigwachstum der Hainbuche ist in der Wipfelregion wie im lateralen Bereich leicht höher als das der untersuchten Buchen und Eschen und liegt im Mittel bei $13.59 \mathrm{~cm}$ pro Jahr. 
Das Dickenwachstum des Stammes ist dagegen mit $11.67 \mathrm{~cm}^{2}$ um $64 \%$ geringer als das der Esche und um 40\% geringer als das der Buche. Hainbuche zeigt den stärksten negativen Wachstumstrend der Trieblängen für den Untersuchungszeitraum $(r=0.93$; s. Tab. 4.6). Nur die Winterlinde zeigt diesen Trend nicht. Sie weist das geringste mittlere Trieblängenwachstum auf und auch der Basalflächenzuwachs liegt mit $12.36 \mathrm{~cm}^{2}$ pro Jahr nur leicht über dem der Hainbuche. Auffällig bei dieser Art ist, dass der Zuwachs im Wipfelbereich sehr viel niedriger ist als der der anderen drei Baumarten (14.27 cm gegenüber 18.45 - $22.66 \mathrm{~cm}$ ). Das laterale Zweigwachstum mit $11.60 \mathrm{~cm}$ ist jedoch etwas stärker als das der Buchen, Eschen und Hainbuchen $(10.01-10.10 \mathrm{~cm})$. Dadurch liegen für diese Art alle Zuwachswerte sehr dicht zusammen. Dass auch zwischen den Untersuchungsjahren kaum Schwankungen auftreten, ist ein weiteres Spezifikum der Winterlinde, womit sie sich deutlich von den anderen Arten unterscheidet.

Tab. 4.7: Zusammenhang zwischen Basalflächenzuwachs und Trieblängenwachstum für die einzelnen Individuen der vier untersuchten Arten.

\begin{tabular}{c|c|c|c|c|c|c|}
\hline \multirow{2}{*}{ Tree No } & \multicolumn{5}{|c}{ Relationship basal area increment / shoot growth } \\
\cline { 2 - 7 } & \multicolumn{2}{|c|}{ All shoots } & \multicolumn{2}{c|}{ Tree-top } & \multicolumn{2}{c}{ Lateral shoots } \\
\cline { 2 - 7 } & $\mathrm{p}$ & $\mathrm{r}$ & $\mathrm{p}$ & $\mathrm{r}$ & $\mathrm{p}$ & $\mathrm{r}$ \\
\hline Fasy 1 & 0.01 & 0.89 & - & - & 0.02 & 0.84 \\
Fasy 2 & 0.03 & 0.79 & - & - & - & - \\
Fasy 3 & - & - & - & - & - & - \\
Fasy 4 & - & - & - & - & - & - \\
Fasy 5 & - & - & - & - & - & - \\
Fasy 6 & - & - & - & - & - & - \\
Frex 1 & 0.02 & 0.85 & 0.02 & 0.83 & 0.01 & 0.86 \\
Frex 2 & - & - & - & - & - & - \\
Frex 3 & - & - & - & - & - & - \\
Frex 4 & - & - & $0.02 *$ & $0.86^{*}$ & - & - \\
Frex 5 & - & - & - & - & - & - \\
Frex 6 & - & - & - & - & - & - \\
Cabe 1-6 & - & - & - & - & - & - \\
Tico 1 & - & - & 0.02 & 0.85 & - & - \\
Tico 2 & - & - & 0.02 & 0.82 & - & - \\
Tico 3 & 0.04 & 0.82 & - & - & - & - \\
Tico 4 & - & - & - & - & 0.04 & 0.73 \\
Tico 5 & - & - & - & - & $0.01 *$ & $0.97^{*}$ \\
\hline Tico
\end{tabular}

* 1999 not included in fit 
Durch die geringe interannuelle Schwankung ergibt sich für die Winterlinde für alle untersuchten Individuen ein signifikanter Zusammenhang zwischen Basalflächenzuwachs und dem Längenwachstum zumindest einzelner Kronenbereiche (s. Tab 4.7). Für die Hainbuche war dieser Zusammenhang zwischen Stamm- und Zweigwachstum für keines der untersuchten Individuen signifikant. Bei Buche und Esche wiesen jeweils zwei Individuen einen signifikanten Zusammenhang beider Parameter auf. Dieser ließ sich für einen der Eschentriebe nur unter Ausschluss des Jahres 1999 nachweisen.

\subsubsection{Einfluss des Klimas auf die Zuwachsdynamik}

Bei der Probenahme wurde zufällig aus den lokal im Kronenraum vorherrschenden und herrschenden Individuen einer Art ausgewählt. Die Stammdurchmesser der Untersuchungsbäume liegen zwischen $35 \mathrm{~cm}$ (Hainbuche und Buche) und maximal $72 \mathrm{~cm}$ (Esche; s. Abb. 4.21). Die Mittelwerte liegen jedoch dicht zusammen zwischen $45 \mathrm{~cm}$ (Hainbuche) und $54 \mathrm{~cm}$ (Esche). Es bestehen keine signifikanten Unterschiede zwischen den Arten.

Die durch Auszählung der Jahresringe bestimmten Baumalter weisen für die Hainbuche die größte Spannweite auf. Während alle Hainbuchen recht ähnliche Durchmesser aufwiesen (35 und $51 \mathrm{~cm}$ ), variierte das Alter zwischen 80 und 175 Jahren. So weist die Art mit dem geringsten mittleren BHD das höchste Durchschnittsalter mit 125 Jahren auf. Das Alter der untersuchten Eschen zeigt ebenfalls eine große Spannweite zwischen 80 und 165 Jahren.

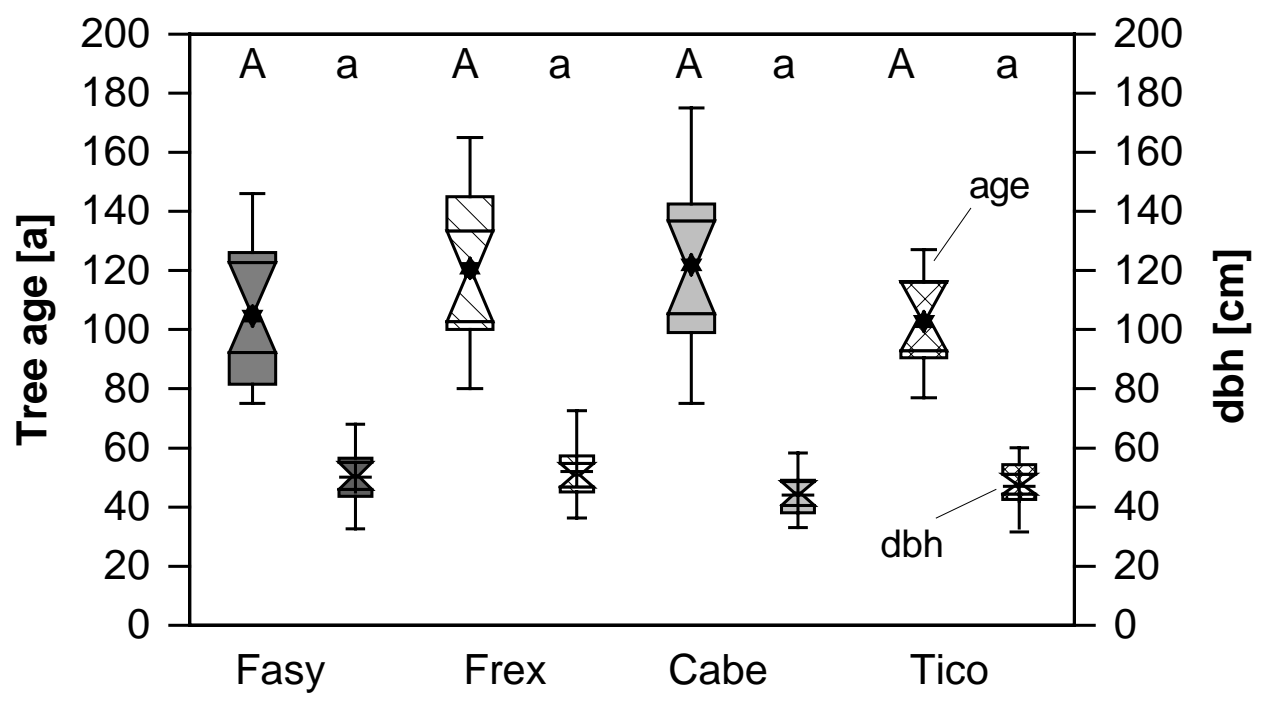

Abb. 4.21: Baumalter und Brusthöhendurchmesser (DBH) der jeweils beprobten Individuen der Arten Buche (Fasy), Esche (Frex), Hainbuche (Cabe) und Winterlinde (Tico) dargestellt in Box-WhiskerPlots. Unterschiedliche Großbuchstaben geben signifikant unterschiedliche Baumalter an, unterschiedliche Kleinbuchstaben kennzeichnen signifikante Unterschiede zwischen den Stammdurchmessern ( $n=15$ je Art; Wilcoxon-U-Test; $p<0.05$ ). 
Die geringste Varianz zeigen die Winterlinden. Die älteste Winterlinde liegt mit 127 Jahren weit hinter den ältesten Individuen der anderen Arten zurück und ist mit durchschnittlich 104 Jahren die jüngste Art im Bestand. Das Durchschnittsalter der beprobten Buchen liegt mit 105 Jahren knapp darüber. Insgesamt zeigt sich auch für das Baumalter kein signifikanter Unterschied zwischen den Arten.

Aus den Einzelkurven wurden Mittelkurven für jede Art berechnet, die einen guten Einblick in die langfristige Zuwachsentwicklung geben (Abb. 4.22). Nach WORBES (1995) verlaufen die Trends der Zeitreihen von Grundflächen- und Radialzuwachs bei älteren Bäumen gleichsinnig, sodass hier für die Darstellung als Einheit die ursprünglich gemessenen Jahrringbreiten und nicht der Flächenzuwachs gewählt wurde.

Hainbuchen und Eschen weisen bis in die Mitte der 50er Jahre des letzten Jahrhunderts sehr ähnliche Ringbreiten um $1.5 \mathrm{~mm}$ auf, die Schwankungen verlaufen größtenteils parallel. Ab den 60er Jahren fällt das Wachstum der Hainbuche kontinuierlich bis zum Messjahr 2004 auf $0.7 \mathrm{~mm}$ ab. Die Esche steigert ihr Wachstum im gleichen Zeitraum drastisch mit einem kleineren Rückgang in den 70er Jahren. In den letzten zwei Jahrzehnten liegen die durchschnittlichen Jahrringbreiten bei $2.5 \mathrm{~mm}$ und damit um den Faktor 3.6 höher als die der Hainbuchen.

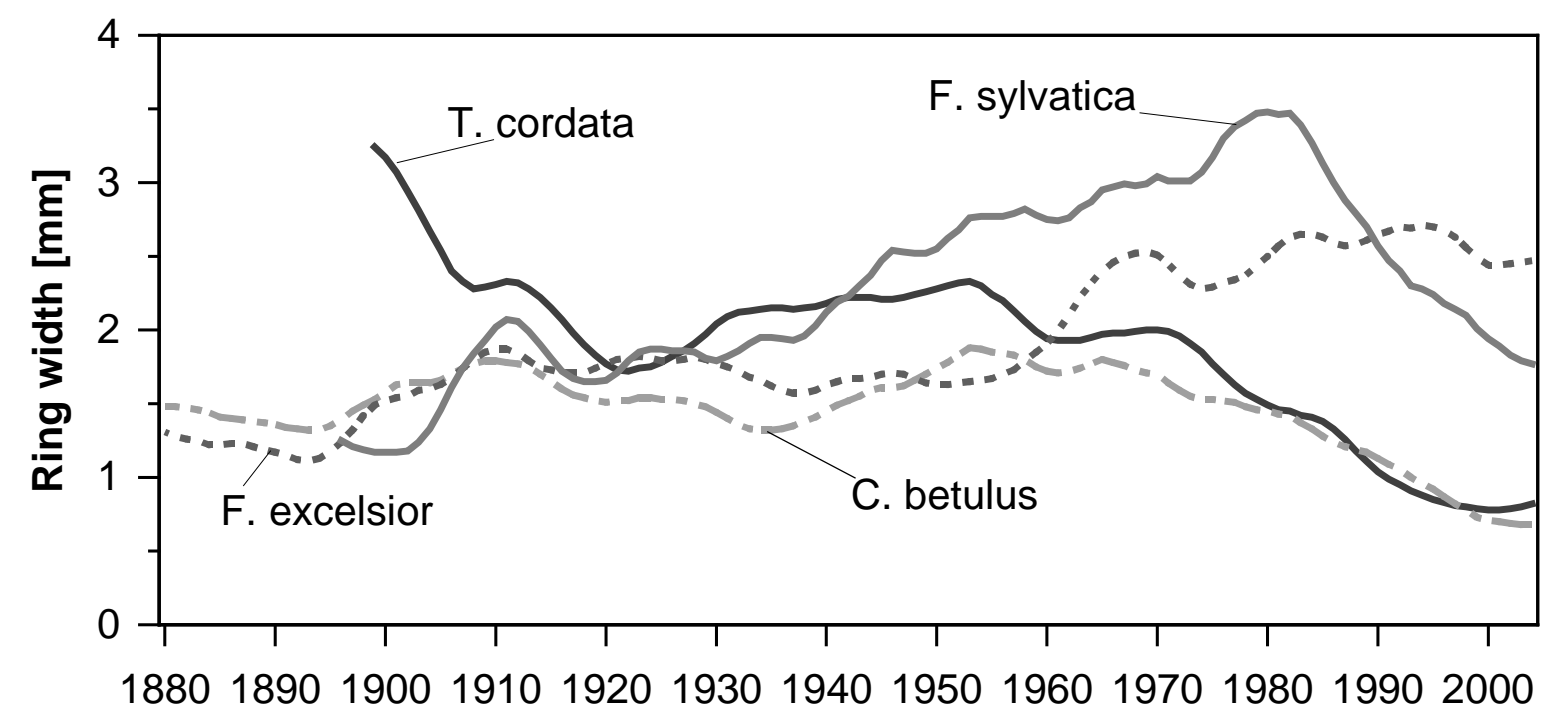

Abb. 4.22: Zuwachsentwicklung der vier untersuchten Baumarten dargestellt als 11-jähriges gleitendes Mittel ( $n=15$; Kurvenbeginn bei einer Belegung von $n=5)$.

Die Buche steigert ihren Stammzuwachs ab 1920 bis 1980 kontinuierlich von $1.6 \mathrm{~mm}$ auf 3.5 $\mathrm{mm}$ und erreicht damit den Spitzenwert für alle Arten. In den letzten zwei Jahrzehnten zeigt sich jedoch ein drastischer Rückgang der Jahrringbreiten, der sich bis in die Gegenwart fortsetzt. Ab den 70er Jahren zeigt sich auch für die Winterlinde ein deutlicher Rückgang der 
Zuwächse, der mit dem der Hainbuche gleichläuft. Im Gegensatz zu Hainbuche und Buche lässt sich jedoch für die Winterlinde ab Ende der 90er Jahre eine Umkehr dieses Trends erkennen, der zu leichten Zuwachssteigerungen bis zum Jahr 2004 führt.

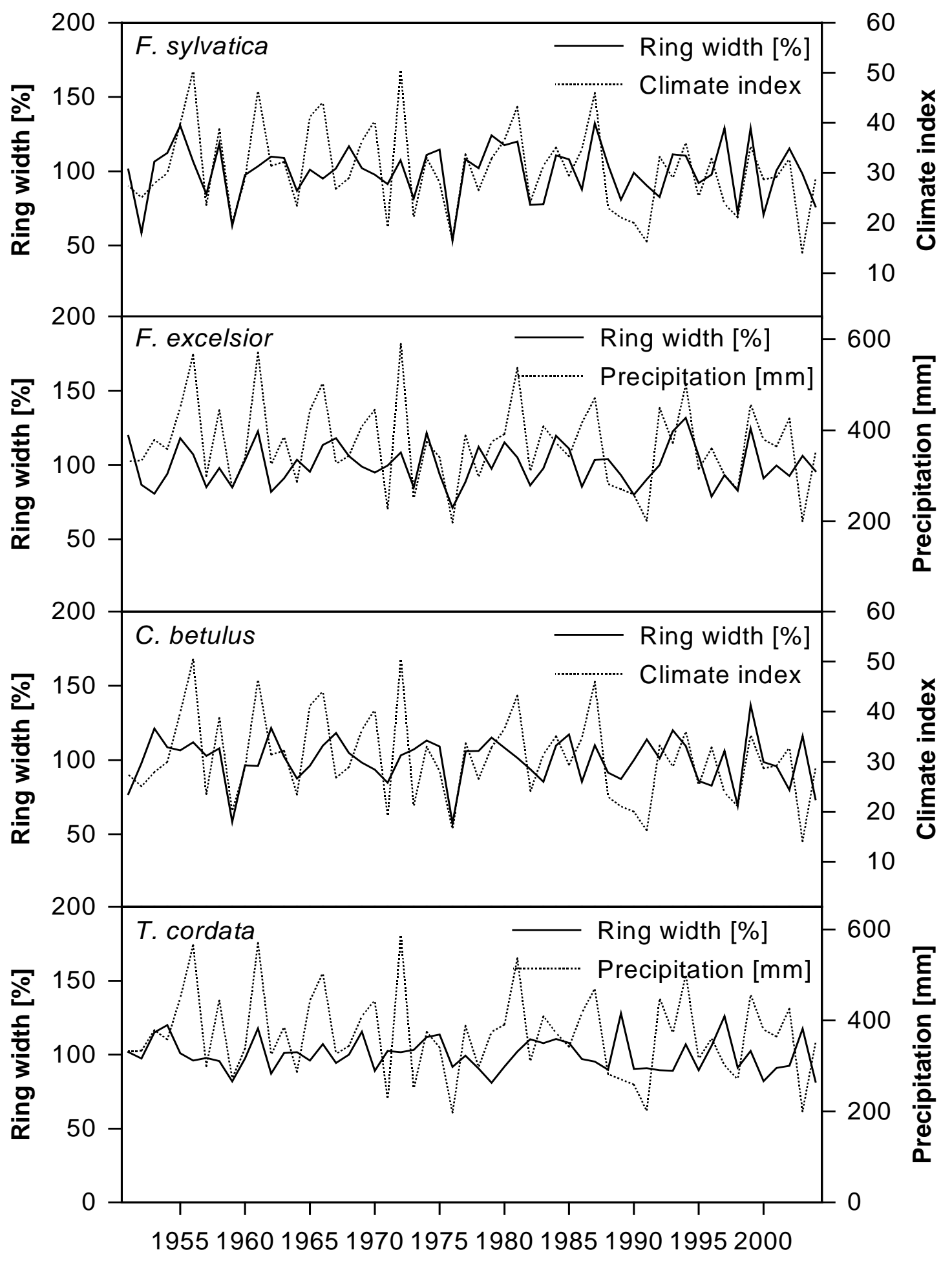

Abb. 4.23: Indexierte Chronologien der vier Baumarten als relativer Zuwachs [\%] vom 11-jährigen Mittel und dem Klimaparameter, der am besten mit der jeweiligen Chronologie korreliert (rechte YAchse). Für Buche und Hainbuche ist dies ein Klimaindex (Quotient aus der Niederschlagssumme März-August und mittlerer Temperatur dieses Zeitraums), für Esche und Winterlinde die Niederschlagssumme der Vegetationsperiode von März bis August. 
Um festzustellen, inwiefern die Zuwachsschwankungen vom lokalen Klima beeinflusst sind, wurden die jährlichen relativen Abweichungen vom gleitenden Mittel der Standortchronologie mit verschiedenen Klimaparametern korreliert. Getestet wurden Jahresniederschlag, Niederschlag in der Vegetations-Periode (von März bis Juli, März bis August, April bis Juli bzw. April bis August), Niederschlag der Vegetationsperiode des Vorjahres und ein Klima-Index, der der Quotient aus Niederschlag und Temperatur der Vegetationsperiode ist. In Abbildung 4.23 sind die Residuen der indexierten Chronologien gegen den Parameter aufgetragen, der die beste Korrelation für die jeweilige Baumart ergab. Die Korrelationen sowie die Regressionsgleichungen sind in Abbildung 4.24 dargestellt. Für Buche und Hainbuche lieferte der Klimaindex aus der Niederschlagssumme und der mittleren Temperatur der Vegetationsperiode (März - August) desselben Jahres die besten Werte.

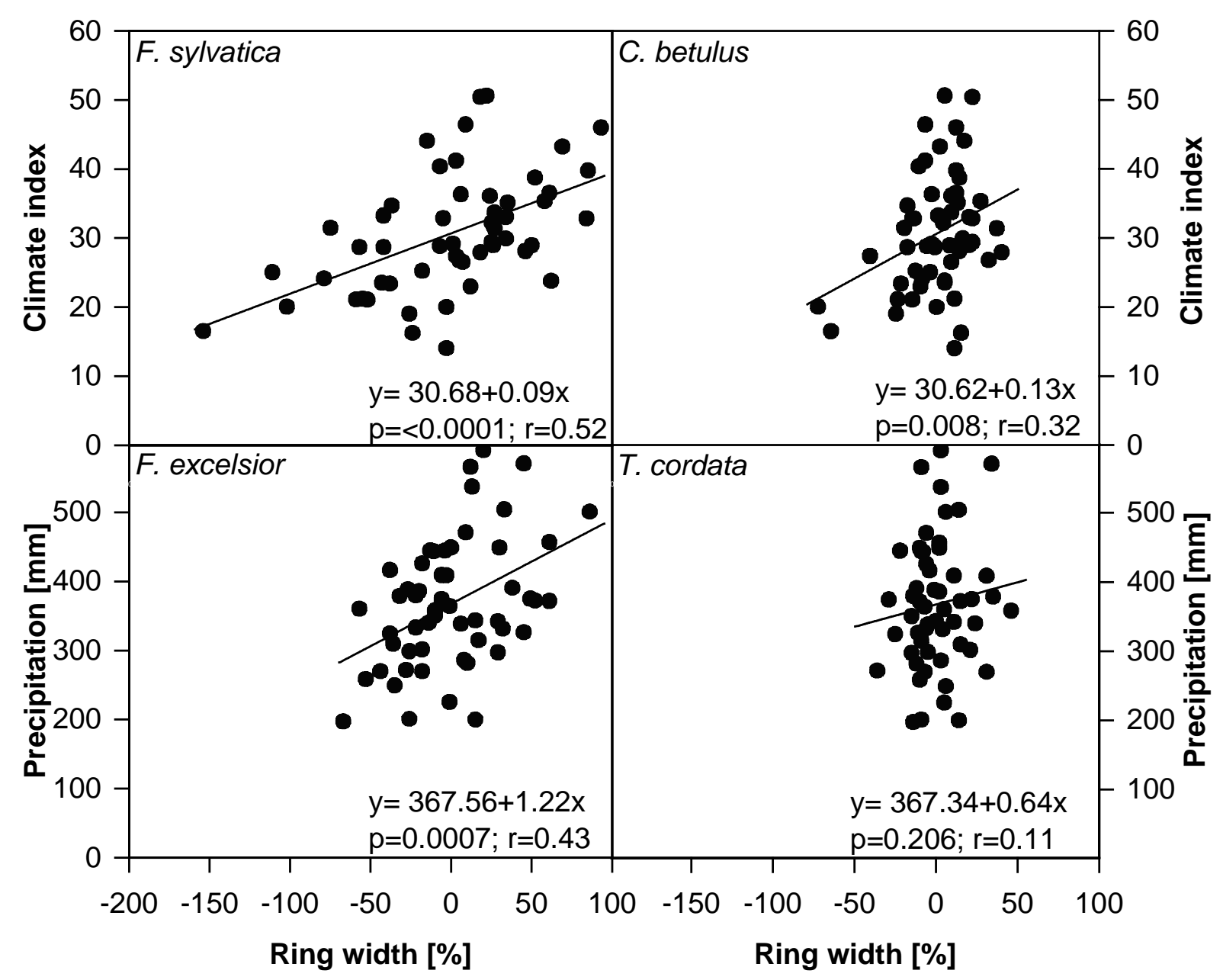

Abb. 4.24: Korrelation der indexierten Chronologien als relative Ringweite in Bezug auf das 11jährige gleitende Mittel mit dem Niederschlag innerhalb der Vegetationsperiode von März bis August (Esche und Winterlinde), bzw. mit einem Klimaindex aus Niederschlag der Vegetationsperiode und Temperatur des gleichen Zeitraums (Buche und Hainbuche). 
Mit einem Korrelationskoeffizienten von 0.52 weist die Buche den engsten Zusammenhang zwischen Klimaindex und Zuwachs auf. Für Hainbuche ist er deutlich schwächer mit $r=0.32$, aber dennoch signifikant. Für die untersuchten Eschen erklärt der Einfluss des Niederschlags der Vegetationsperiode (März bis August) 18.5\% der Gesamtvarianz.

Die Winterlinde zeigt als einzige Baumart keinen signifikanten Zusammenhang zwischen Stammzuwachs und Klima. Die Ringbreiten sind sehr viel stärker abhängig von der Ringbreite des Vorjahres als von jährlichen Schwankungen der Wachstumsbedingungen. Statistisch lässt sich das mit der hohen Autokorrelation und der geringen Sensitivität dieser Art belegen (s. Tab. 4.8). Bei der Autokorrelation wird die jeweilige Jahrringbreite mit der Ringbreite des Vorjahres korreliert. Je stärker diese Korrelation ist, desto kontinuierlicher verläuft das Wachstum. Mit 0.89 weist die Winterlinde den höchsten Wert der vier untersuchten Baumarten auf. Für die Buche liegt er mit 0.7 am niedrigsten. Umgekehrt proportional dazu verhält sich die Sensitivität, bei der die Intensität der interannuellen Schwankung einer Einzel- oder Mittelkurve berechnet wird. Hier liegt der Wert für die Winterlinde am niedrigsten (0.12), gefolgt von der Hainbuche (0.16) und der Esche (0.17) bis hin zur Buche (0.2).

Tab. 4.8: Vergleich der Ringweiten, der Autokorrelation mit der Ringweite des Vorjahres (AC 1) und der mittleren Sensitivität (MS) der in Abb. 4.22 dargestellten Mittelkurven für die vier untersuchten Baumarten.

\begin{tabular}{l|ccc|cc}
\hline Species & \multicolumn{3}{|c|}{ Ring width [mm] } & \multicolumn{2}{c}{ Internal statistics } \\
\hline \multirow{2}{*}{ F. sylvatica } & Min & Mean & Max & AC(1) & MS \\
\cline { 2 - 6 } F. excelsior & 0.79 & 2.30 & 4.31 & 0.70 & 0.20 \\
C. betulus & 0.72 & 1.79 & 3.57 & 0.78 & 0.17 \\
T. cordata & 0.50 & 1.47 & 2.28 & 0.69 & 0.16 \\
\hline
\end{tabular}




\section{Diskussion}

Die Diskussion der vorliegenden Ergebnisse orientiert sich an der einleitend beschriebenen doppelten Zielstellung, bei der einerseits eine flächenbezogene Analyse der Bestandesstruktur eine Einschätzung der potenziellen weiteren Bestandesentwicklung geben soll und andererseits eine baumartenbezogene Analyse Unterschiede in der Autökologie der untersuchten Arten sowie in den Nachbarschafts-Interaktionen aufzeigt.

Im ersten Teil steht der Flächenbezug im Vordergrund. Anschließend wird der Baumartenbezug im Mittelpunkt stehen, wobei von den räumlichen Interaktionen auf der Individuen-Ebene ausgehend dieses Thema auf der Ast-Ebene vertieft wird.

Im letzten Teil soll unter Einbeziehung der dendroökologischen Daten eine abschließende Charakterisierung der untersuchten Arten und ihrer artspezifischen Unterschiede hinsichtlich ihrer Wuchsform, Klimasensitivität und Kompatibilität innerhalb des Mischwaldes gegeben werden.

\subsection{Bestandesstruktur und Bestandesentwicklung -}

\section{Artenverschiebungen zwischen Totholz, Baumschicht und}

\section{Verjüngung}

5.1.1 Wald- und Forstgeschichte als Ursache der heutigen Bestandesstruktur

Die vier Untersuchungsflächen liegen innerhalb des Nationalparks Hainich in drei verschiedenen Waldbereichen mit teilweise unterschiedlicher Bestandesgeschichte. Die Flächen 1 und 2 befinden sich im ,Lindig,, in dem bis zum Zweiten Weltkrieg die historisch in diesem Gebiet weit zurückreichende Nutzungsform der Mittelwaldwirtschaft beibehalten wurde (Отто 2000). Davon zeugen die deutlich größeren Stammumfänge der Buchen und Stieleichen auf diesen Flächen (s. Abb. 4.1), die als Überhälter gefördert und regelmäßig frei gestellt wurden. Durch diese Wirtschaftsweise wurden in der Hauschicht Baumarten gefördert, die sich gut aus Stockausschlägen regenerieren, was den hohen Anteil an Linden und Eschen erklären würde, aber auch das erstaunlich häufige Auftreten des Feldahorns, der im geschlossenen Bestand sehr selten anzutreffen ist. Diese Nutzungsform führte zu einer Verdrängung der Buche in der Hauschicht, da diese kaum Stockausschläge bildet, und begünstigte andere Laubbaumarten (KÜSTER 1996). 
Fläche 3 liegt im Langensalzaer Stadtwald, der größten Teils bis ins 20. Jahrhundert hinein von einer Laubgenossenschaft im Plenterwaldbetrieb genutzt wurde (KLAUS \& REISINGER 1995). Beim Umbau des Mittelwaldes in Hochwald für die Plenterwaldwirtschaft wurde verstärkt auf kernwüchsiges Oberholz und einer Anreicherung des Bestandes mit Edellaubhölzern gewirtschaftet (Отто 2000). Die im Vergleich zu den Flächen 1 und 2 sehr viel homogenere BHD-Verteilung und die hohe Artendurchmischung (s. Tab. 4.1) auf Fläche 3 zeugt von einer einzelstammweisen Nutzung. Zudem ist das Auftreten von starken Elsbeeren und Kirschen auf dieser Fläche (s. Abb. 4.1) ein deutliches Zeichen der früheren Wirtschafts- und Pflegeziele.

Durch die kleinräumig wechselnden Besitzverhältnisse im Hainich ist die Bestandesgeschichte der Fläche 4, die sich im Alterstedter Holz befindet, schwer rekonstruierbar. Der von Buchen dominierte Bestand zeigt eine deutlich andere Struktur als die Flächen im Lindig und deutet eher auf eine frühere plenterartige Nutzung hin. Charakteristisch sind einige sehr starke Eschen mit Durchmessern zwischen 86 und $126 \mathrm{~cm}$, was einem Umfang von $2.70 \mathrm{~m}$, bzw. 4 m entspricht.

Alle vier Flächen sind in ihrer Struktur und ihrer Baumartenzusammensetzung stark anthropogen geprägt. Die Entwicklung der letzten 50 Jahre blieb allerdings relativ wenig vom Menschen beeinflusst, da der gesamte Bestand nach dem Zweiten Weltkrieg zwar zunächst durch sowjetische Truppen aufgeteilt und an landarme Bauern verteilt wurde, jedoch 1961 zum Naturschutzgebiet erklärt wurde. Drei Jahre später übernahm die Nationale Volksarmee das Waldgebiet und die angrenzenden Freiflächen. Seit dieser Zeit erfolgte keine gezielte Pflege der Bestände mehr, jedoch muss von einer bedarfsorientierten, regellosen Nutzung durch die Anwohner in den umliegenden Dörfern ausgegangen werden (Отто 2000).

Die Intensität dieser Nutzung ist nicht dokumentiert, als sicher kann nur gelten, dass keine gezielte Pflege oder Nutzung nur einer Baumart betrieben wurde. Somit spiegeln die heutigen Verhältnisse im Untersuchungsgebiet den Stand einer in den letzten Jahrzehnten relativ ungestörten walddynamischen Entwicklung mitsamt der sich dabei abspielenden Konkurrenzprozesse wider.

\subsubsection{Baumschicht und Verjüngung}

Bis auf eine durch Windwurf im Jahr 2002 entstandene Kronenlücke am Rand der Fläche 1 weisen alle vier Untersuchungsflächen eine geschlossene Kronenschicht auf. Die daraus resultierende einheitlich niedrige Beleuchtungsstärke am Waldboden zwischen 2.3\% und $1.3 \%$ der Freiland-Strahlung (s. Abb. 4.2) beeinträchtigt die Etablierung der Jungpflanzen 
aller Baumarten und ermöglicht nur den schattentoleranten Sämlingen spätsukzessionaler Baumarten ein Überleben (CANHAM et al. 1994). Die in der Baumschicht häufig vertretenen Arten sind Schlusswaldarten (Buche, Winterlinde, Esche, Hainbuche, Berg- und Spitzahorn), ihre Sämlinge sind somit an geringe Strahlung am Waldboden angepasst (ELLENBERG 1996). Dennoch verjüngen sich die Arten innerhalb dieser funktionellen Gruppe sehr unterschiedlich erfolgreich. Die Anteile der Arten in der Baumschicht stehen in keinem Verhältnis zu den Abundanzen in der Verjüngung (s. Abb. 4.3). Auffällig ist zum Einen die starke Verjüngung der Esche sowie des Berg- und Spitzahorns und andererseits das Fehlen einer nennenswerten Verjüngung von Winterlinden und Buchen selbst auf den Flächen, auf denen sie mit über 50\% in der Baumschicht vertreten sind.

Die hohe Verjüngungsdichte der Esche von 15 Individuen pro $\mathrm{m}^{2}$ deckt sich mit Ergebnissen von WAGNER (1999), der in Buchenmischwäldern eine Dichte von 16 Individuen pro $\mathrm{m}^{2}$ nachwies, wobei die mittlere Ausbreitungsdistanz der Eschen bei $40 \mathrm{~m}$ vom jeweiligen Mutterbaum lag. In Eschenmischwäldern in Großbritannien belegt OKALI (1966) Verjüngungsdichten der Eschen von bis zu 20 Individuen pro $\mathrm{m}^{2}$. Der gleiche Autor berichtet jedoch auch von einer überproportional hohen Bergahornverjüngung (2.78-6.88 Ind. $/ \mathrm{m}^{2}$ ). Diese Art zeigt sich in England als invasiv und besiedelt zunehmend Eschenstandorte. Aufgrund der sehr effizienten Verbreitungsmechanismen (JOHNSON 1988) ist auch bei dieser Art die Verjüngungshäufigkeit nicht mit der Anzahl samenproduzierender Altbäume im Bestand korreliert (OKALI 1966). Das Phänomen überproportional erhöhter Verjüngungsdichten dieser beiden Arten beschreiben auch HÉRAULT et al. (2004) für Fichtenplantagen in Luxemburg und MODRÝ et al. (2004) für Buchenwälder in Tschechien.

CANHAM (1994) führt die starken Unterschiede in der Verjüngungsdichte innerhalb der spätsukzessionalen Arten auf Unterschiede der Ressourcennutzung und der Schattentoleranz zurück, die die sekundäre Sukzession steuern. Auch HÄTTENSCHWILER (2001) weist auf die große Bandbreite an morphologischen und physiologischen Eigenschaften innerhalb der spätsukzessionalen Arten hin. Seinen Experimenten mit unterschiedlichen Beleuchtungsregimen von Jungpflanzen verschiedener Baumarten nach, ist Bergahorn der Buche im Höhenwachstum bei geringem Lichtgenuss (1.3\% der Freilandstrahlung) überlegen. Bergahorn zeichnet sich ebenfalls durch sehr gute Überdauerungsraten im Bestandesschatten aus. OKALI (1966) beschreibt die Ähnlichkeit der Esche und des Bergahorns hinsichtlich ihrer Verbreitungsbiologie und ihren Standortansprüchen. Die Überlegenheit dieser Arten gegenüber der Buche in der Etablierungsphase gilt allerdings nur für frische, nährstoff- und basenreiche Standorte (MODRÝ 2004, KERR \& CAHALAN 2004). 
Die Gegebenheiten im Hainich entsprechen dieser Merkmalskombination von guter Wasserund Nährstoffversorgung und geringer Strahlung am Waldboden. Letztere variiert jedoch kleinräumig stark, was einerseits auf kleinere Kronenlücken zurückzuführen ist. Diese haben allerdings geringe Ausmaße von maximal $50 \mathrm{~m}^{2}$ (was der Hälfte einer mittleren Kronenfläche der Altbäume entspricht) und haben einen Flächenanteil von $8.1 \%, 5.0 \%, 2.6 \%$ und 2.8\% (Flächen 1 bis 4), was nach MEYER et al. (2003) als sehr gering einzustufen ist, mit Ausnahme der $8.1 \%$ auf Fläche 1. Der hier leicht erhöhte Lückenanteil ist auf den bereits erwähnten Windwurf am Flächenrand zurückzuführen. Durch seine Lage am Flächenrand hatte er auf die Verjüngungsaufnahmen und Lichtmessungen keinen Einfluss.

Eine zusätzliche Heterogenität wird durch die unterschiedliche Transmissivität der Kronen der unterschiedlichen Arten bewirkt. Nach HAGEMEIER (2002) haben Linden im Reinbestand mit $1.3 \%$ der Freilandstrahlung genauso niedrige Transmissionsraten wie Buchenbestände. Hainbuchen liegen mit $1.4 \%$ nur knapp darüber. Die höheren Strahlungswerte auf den winterlindenreichen Fläche 1 und 2 (Abb. 4.2) gegenüber der von Buchen dominierten Fläche 4 sind demnach nicht auf den Dominanzwechsel der Arten zurückzuführen, sondern beruhen auf dem hohen Anteil an Eschen- und Eichenbeimischung. Für Eichen ermittelte HAGEMEIER (2002) eine Kronentransmissivität von 8.4\%. Eschenkronen sind aufgrund ihres geringen LAI ebenfalls sehr lichtdurchlässig. HÖLSCHER (2003) ermittelte für Eschen auf einer benachbarten Untersuchungsfläche im Hainich einen LAI von 3.5, wohingegen Linden einen LAI von 5.1 aufwiesen.

Unter den lichtdurchlässigeren Kronen von Eschen und Eichen kommt es jedoch zu keiner deutlich veränderten Artenzusammensetzung in der Verjüngung. Lediglich die Winterlinde ist unter Eichenschirmen signifikant häufiger vertreten als unter anderen Baumarten (vgl. Abb. 4.5). Der unter Eichen ebenfalls häufig auftretende Spitzahorn ist unter der sehr dicht belaubten Sommerlinde in etwa ebenso stark vertreten. Nach RUNKLE (1981) ist in einem stark durchmischten Bestand keine räumliche Separation einzelner Arten unter den Schirmen spezieller Altbäume zu erwarten. Auch KNOERZER (2002) wies für Douglasienverjüngung nach, dass die am Waldboden ankommende Lichtmenge entscheidender für die Etablierung der Jungpflanzen ist als die Artzusammensetzung des Altbestandes. CANHAM et al. (1994) stellten bei Jungbäumen unterschiedlich schattentoleranter Arten (Fagus grandifolia, Tsuga canadensis, Fraxinus americana und Quercus rubra) jedoch fest, dass alle untersuchten Arten höhere Überlebenswahrscheinlichkeiten bei Überschirmung von Altbäumen ihrer eigenen Art aufwiesen. Dieser Zusammenhang konnte von HosHINO et al. (2001) für artenreiche Laubwälder in Japan nicht nachgewiesen werden. 
Mit Ausnahme der Fläche 3 wurde keine Korrelation zwischen der Verjüngungsdichte und der lokalen relativen Beleuchtungsstärke gefunden. Auch auf dieser war der Zusammenhang mit einem Korrelationskoeffizienten von $\mathrm{r}=0.37$ nur sehr schwach ausgeprägt und erklärt nur 13\% der Gesamtvarianz. Dieses Ergebnis deckt sich mit anderen Untersuchungen der Verjüngungsprozesse spätsukzessionaler Arten in Laubmischwäldern. (z.B. CATOVSKY \& BAZZAZ 2002, MEYER et al. 2003, MODRÝ 2004) und wird mit der hohen Schattentoleranz der Jungbäume erklärt. Neben Lichtentzug können Altbäume die Verjüngung jedoch auch durch Wurzelkonkurrenz und Beeinflussung des pH-Werts des Bodens durch die anfallende Laubstreu beeinflussen. Da sich die Laubstreu jedoch in diesen Beständen stark mischt und sich nicht ausschließlich unter der eigenen Krone ansammelt, könnte sich ein solcher Einfluss nicht als Verteilungsmuster in der Verjüngung niederschlagen. Auch die Wurzeln eines Altbaumes reichen zum Teil weit über die Kronenschirmfläche des Altbaumes hinaus (KORN 2004). Zudem wird die unterirdische Konkurrenz in Wäldern mit guter Wasser- und Nährstoffverfügbarkeit für die Etablierung von Jungpflanzen als weniger ausschlaggebend beurteilt als die Strahlungsverhältnisse am Waldboden (COOMS \& GRUBB 2000).

\subsubsection{Verbiss durch Wild}

Neben den abiotischen Faktoren und dem Einfluss der Altbäume auf die Verjüngung ist der Wildverbiss eine Einflussgröße von nicht zu unterschätzender Wichtigkeit. Der seit Einrichtung des Nationalparks durch gestiegene Wilddichte (GOHRBANDT 2005) noch erhöhte Fraßdruck behindert die Regeneration der Baumschicht erheblich. Ein für die Waldentwicklung sicherlich entscheidender Faktor ist selektiver Fraßdruck. Viele Autoren berichten von einem signifikant stärkeren Verbiss von Eschen und Bergahorn gegenüber Buchen in Buchenmischwäldern (z.B. HARMER 1999, MEYER et al. 2003, MODRÝ 2004). Dies ließ sich auf den hier untersuchten Flächen nicht nachweisen. Buchenschösslinge wurden mit 61.90\% nahezu ebenso oft verbissen wie Berg- und Spitzahorn (72.5 bzw. 72.4\%, vgl. Tab. 4.2). Die Esche trug mit $43.1 \%$ signifikant weniger häufig Verbissspuren. Bei einer Beurteilung dieser Werte muss jedoch die unterschiedliche Häufigkeit der einzelnen Arten beachtet werden, die sich im Falle der Buche auf wenige Individuen pro Fläche beschränken, mit Ausnahme von Fläche 4. Die Verjüngung der Esche hingegen ist stellenweise teppichartig. Dieser dichte Jungwuchs der Esche überschreitet allerdings zum größten Teil nicht die Höhenstufe von $20 \mathrm{~cm}$, wie Abbildung 5.1 zeigt. Der ständige Fraßdruck verhindert ein Emporkommen der Jungpflanzen über ein kritisches Niveau von $30 \mathrm{~cm}$, ab dem die Jungpflanzen als etablierter Jungwuchs angesehen werden können (HARMER 2001, PRETZSCH 
2002). Die Individuenzahlen junger Eschen, Berg- sowie Spitzahorne gehen ab einer Höhe von $20 \mathrm{~cm}$ drastisch zurück. Eine Höhe von $30 \mathrm{~cm}$ erreichen nur wenige Individuen dieser Arten. Eine Höhe von $40 \mathrm{~cm}$ erreichen weder Berg- noch Spitzahorn. Eschen werden vereinzelt auch über $100 \mathrm{~cm}$ hoch.

Die Hainbuche findet sich nur mit wenigen Individuen in der Verjüngung und erreicht ebenfalls die kritische Höhe von $30 \mathrm{~cm}$ nicht. Buchenjungwuchs ist etwas stärker vertreten und zeigt sich auf der buchenreichen Fläche 4 auch mit einigen Individuen über $30 \mathrm{~cm}$.

Die einzige Art, die nicht diesen Rückgang in der Häufigkeit zwischen den Höhenstufen zwischen 20 und $30 \mathrm{~cm}$ aufweist, ist die Winterlinde. Zahlenmäßig im Vergleich äußerst schwach vertreten, kommt sie in allen Höhenstufen vor und erreicht nicht nur durchschnittlich die größte Höhe, sondern auch die größte Stammdicke (vgl. Abb. 4.4).
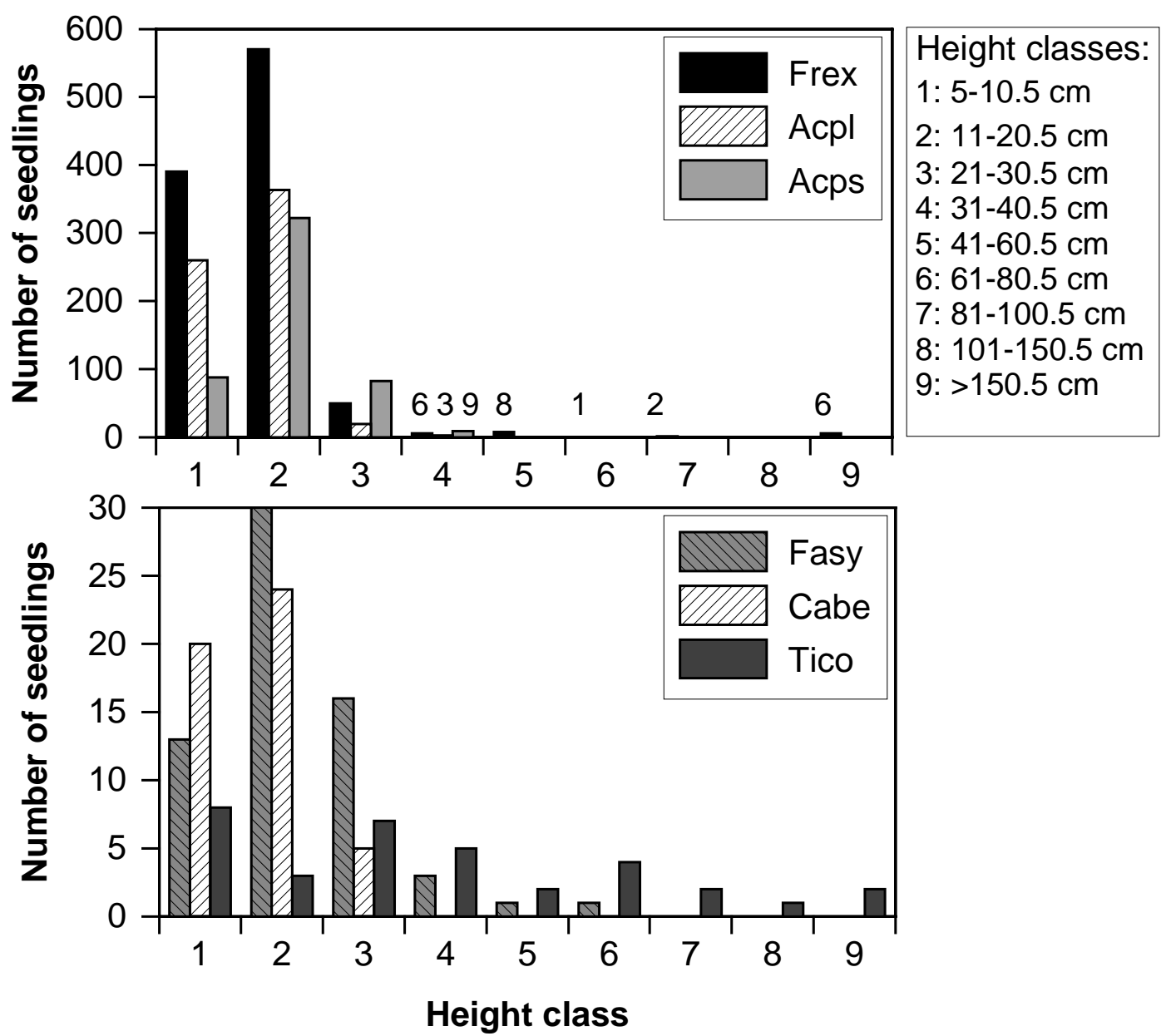

Abb. 5.1: Häufigkeitsverteilung der Jungpflanzen nach Höhenstufen. Oben: Esche (Frex), Spitzahorn (Acpl), Bergahorn (Acps); unten: Buche (Fasy), Hainbuche (Cabe) und Winterlinde (Tico). Die beiden Abbildungen sind auf der Y-Achse unterschiedlich skaliert. 
Auf allen untersuchten Kleinflächen erreichten insgesamt nur 6 Eschen und 3 Winterlinden eine Höhe von über $130 \mathrm{~cm}$, ab der Jungbäume der Gefahr eines Terminaltriebverbisses nicht mehr ausgesetzt sind (RоTH 1996). Sieht man diese Zahlen vor dem Hintergrund der sehr guten Vorverjüngung und den hohen Verbissraten, wird deutlich, dass die Regeneration der untersuchten Bestände massiv vom Wildverbiss beeinflusst wird. Wie resistent Jungbäume gegen Verbiss sind, ist artspezifisch unterschiedlich. HARMER (2001) wies experimentell eine signifikant höhere Überlebensrate von verbissenen Buchen gegenüber Esche und Bergahorn im Bestandesschatten nach. Jedoch führte er diese Versuche an schon etablierten Jungpflanzen durch. In niedrigeren Höhenstufen wirkt sich der Schaden durch Wildverbiss und auch die intensivere Konkurrenz mit der Krautschicht noch stärker aus. Ab wann Verbissschäden in der Verjüngung zu einer Beeinträchtigung der Waldregeneration führen, hängt von vielen Faktoren wie der Sämlingsdichte, dem Lichtgenuss (SENN 2002) und der Mischungsanteile der verbissgefährdeten Baumarten in der Oberschicht der Verjüngung sowie deren Verbissrate ab (Rотн 1996). Bei einer Verbissrate von über 50\% sei nach diesem Autor jedoch von einer nachhaltigen Schädigung der Baumverjüngung auszugehen. Im Nationalpark Hainich wurden für das Jahr 2005 Wilddichten von 13.1 Individuen $/ \mathrm{km}^{2}$ für Rehwild, 3.6 für Damwild und 1.7 Individuen $/ \mathrm{km}^{2}$ für Rotwild ermittelt (GOHRBANDT 2005). Nach einer Untersuchung von RATCLIFFE (1987) führen Rotwilddichten ab 4/km ${ }^{2} \mathrm{zu}$ erheblichen waldbaulichen Schäden. Betrachtet man den Anteil von Dam- und Rotwild zusammen, so scheint im Nationalparkgebiet eine kritische Dichte erreicht zu sein. Die Beurteilung der Waldschäden durch Großäser wird jedoch sehr kontrovers diskutiert (ROTH 1996). Die Dichte allein ist nach RADELOFF (1999) nicht entscheidend; der Habitattyp und die Baumartenzusammensetzung sind ebenfalls entscheidend. Aber auch bei sehr guter Habitatqualität liegt die ökonomisch akzeptable Wilddichte für Rehwild bei 11 Individuen pro Quadratkilometer (UECKERMANN 1988). Die maximale Wilddichte, also die Populationsdichte, bei maximaler Ausschöpfung der ökologischen Habitatkapazität gibt der Autor mit 13$15 / \mathrm{km}^{2}$ an. Auch der Rehwildbestand muss demnach als hoch angesehen werden. Er entspricht aber der regionalen Wilddichte. Diese wird von WEBER (2003) ebenfalls als kritisch hoch und gefährdend für eine natürliche Regeneration der Waldbestände bewertet.

\subsubsection{Regenerationsvermögen der einzelnen Arten}

Die auffällig gute Etablierung der Winterlinden-Verjüngung kann auf eine von den anderen bestandesbildenden Arten abweichenden Reproduktionsstrategie zurückgeführt werden. Alle etablierten Junglinden stammten als Wurzelschösslinge oder Stockausschläge meist in 
unmittelbarer Nähe zu Altbäumen eindeutig aus vegetativer Vermehrung. Die bessere Ressourcenverfügbarkeit durch ein bedeutend größeres Wurzelsystem erhöht die Überlebenschancen von Wurzelschösslingen im geschlossenen Bestand und bei starkem Verbiss (Verbissrate der Linde: 80\%) erheblich. Diese Art der Verjüngung, die auch LeIBUNDGUT (1991) und NAMVAR \& SPeTHMANN (1986) bei der Winterlinde beobachteten, gleicht ihre etwas geringere Schattentoleranz und das hinter Eschen und Ahorn zurückbleibende Höhenwachstum aus (ELLENBERG 1996). Durch die meist unmittelbare Nähe zum Stamm eines Altbaumes kann es sein, dass der relative Anteil an Winterlinden in der Verjüngung durch die angewandte Inventur-Methode unterschätzt wurde. Lag innerhalb einer zufällig ausgewählten Kleinfläche von $1 \mathrm{~m}^{2}$ der Stamm eines starken Altbaumes, so wurde der Plot um einen Meter verschoben, da sonst die Grundfläche des Altbaumes den größten Teil der Untersuchungsfläche eingenommen hätte. Damit wurden Stockausschläge im Verhältnis weniger erfasst.

Im Bestand ließ sich an vielen Stellen beobachten, wie aus dem Stamm umgestürzter toter wie noch lebender Linden eine große Zahl von Schösslingen wuchsen. Diese haben gegenüber angesamten Jungpflanzen in der Nähe einen großen Vorsprung und damit die besten Chancen, die entstandene Kronenlücke als erste zu erreichen. Auch ScHÖNE (2004) fand in einem Laubmischwald mit einer ähnlichen Baumartenzusammensetzung sehr gute Etablierungsraten für Winterlinden-Jungpflanzen. Die auch in diesem Bestand dominierende Ahornverjüngung (94\% der Gesamtverjüngung) ging ebenfalls ab einer Höhenstufe von $50 \mathrm{~cm}$ drastisch zurück.

So kann davon ausgegangen werden, dass die Winterlinde ihre Präsenz in der Baumschicht auch weiterhin erhalten kann. Auch die Esche scheint gute Chancen zu haben, sich im Bestand verjüngen zu können. Ob der hohe Anteil von Berg- und Spitzahorn in der Vorverjüngung dazu führen wird, den bisher kleinen Anteil an den oberen Bestandesschichten auszubauen, hängt wohl stark von der zukünftigen Wilddichte, bzw. deren Schwankungen ab.

Die sehr schwache Verjüngung der Rotbuche auf den Flächen 1 und 2 wird vermutlich nicht ausreichend sein, um die Häufigkeit dieser Art auf den Lindig-Flächen zu erhalten oder gar auszubauen. Auf der buchenreichen Fläche 4 hingegen zeigt sich eine gute Buchenverjüngung. Die Winterlinde ist hier entgegen den geringen Vorkommen in der Verjüngung mit Jungbäumen von über 3 Metern häufig vertreten. Da diese bei der Verjüngungsinventur nicht mehr erfasst wurden, die Aufnahmen der Baumschicht aber erst Bäume mit einem BHD $>7 \mathrm{~cm}$ berücksichtigten, die deutlich höher sind, wurden die Jungbäume der Arten Buche und Winterlinde auf Fläche 4 gesondert aufgenommen. Nur auf 
dieser Fläche fand sich eine größere Anzahl von Bäumen dieser Bestandesschicht. Auf der ganzen Fläche wurden 50 Winterlinden und 4 Buchen erfasst. Dieses Verhältnis entspricht nicht der Häufigkeitsverteilung in der Kronenschicht (52\% Buche, 25\% Winterlinde). Die sehr starke Präsenz der Winterlinde im Unterwuchs wird allerdings dadurch relativiert, dass $42 \%$ der erfassten Jungbäume nicht aufrecht standen, sondern der Stamm nach unten gebogen war und der Terminaltrieb nach unten wies (s. Tab. 5.1). Ein solches überproportionales Höhenwachstum gegenüber dem Dickenwachstum, das zu einer Destabilisierung des Baumes führt, entsteht unter Lichtmangel und zeigt die geringere Schattentoleranz der Winterlinde gegenüber der Buche in der juvenilen Phase (GIA 1927, LYR et al. 1964).

Tab. 5.1: Anzahl der Jungbäume von Rotbuche und Winterlinde auf Fläche 4

\begin{tabular}{lcccc}
\hline & $\begin{array}{c}\text { No of trees } \\
<7 \mathrm{~cm} \mathrm{DBH}\end{array}$ & $\begin{array}{c}\text { bend down trees } \\
{[\%]}\end{array}$ & $\begin{array}{c}\text { bend down with } \\
\text { reiteration [\%] }\end{array}$ & $\begin{array}{c}\text { dead } \\
{[\%]}\end{array}$ \\
\hline $\begin{array}{l}\text { Fagus sylvatica } \\
\text { Tilia cordata }\end{array}$ & 4 & 0 & 0 & 25 \\
\hline
\end{tabular}

Knapp die Hälfte aller gebogenen Linden wiesen zudem Reiterationen auf. Als Seitenzweige angelegte Triebe, die durch die Beugung des Stammes in senkrechte Position geraten sind, übernehmen dabei die Funktion einer neuen Sprossachse. Dieses Phänomen zeigt an, dass der Verlust der Apikaldominanz, die für ein schnelles Höhenwachstum und damit für eine Etablierung im Kronenraum wichtig ist, bei diesen Individuen irreversibel ist (AARSSEN 1995). Bei jungen Buchen wurde dieses Phänomen nicht beobachtet.

\subsubsection{Totholz}

Das Vorkommen an liegendem und stehendem Totholz ist ein wichtiger Indikator für die jüngste Bestandesgeschichte und für die Beurteilung der Naturnähe eines Bestandes (FALINSKI 1986, BoBIEC 2002). FALINSKI (1978 zit. nach KIRBY et al. 1998) ermittelte für ein naturnahes Tilio-Carpinetum im Nationalpark Białowieza Totholzvolumen von $60-71 \mathrm{~m}^{3} \mathrm{ha}^{-1}$. KIRBY et al. (1998) erhoben im gleichen Gebiet Werte zwischen 75 und $94 \mathrm{~m}^{3} \mathrm{ha}^{-1}$. Durch das unregelmäßige Auftreten von Störungen wie Windwurf oder Trockenperioden schwankt der Totholzvorrat auch in nicht bewirtschafteten Wäldern erheblich. So ermittelte MOUNTFORD (2002) für einen Kalk-Buchenwald in Nordfrankreich $1982103 \mathrm{~m}^{3} \mathrm{ha}^{-1}$ und nach häufigen Sturmereignissen in dieser Region in den 90er Jahren im Jahr $2000256 \mathrm{~m}^{3} \mathrm{ha}^{-1}$. In einer Zusammenschau gibt KIRBY (1998) für unbewirtschaftete, naturnahe Laubwälder in Europa 
Durchschnittswerte von $46-137 \mathrm{~m}^{3} \mathrm{ha}^{-1}$ an. Dies deckt sich weitgehend mit Angaben von MEYER et al. (2003), der in einem Datenvergleich verschiedener Autoren Werte zwischen 50 und $200 \mathrm{~m}^{3} \mathrm{ha}^{-1}$ und einen Anteil von 10-35\% am Gesamtvorrat der Holzbiomasse als typisch für europäische Urwälder angibt.

Der Totholzvorrat auf den hier untersuchten Flächen liegt zwischen 4.8 und $25.6 \mathrm{~m}^{3} \mathrm{ha}^{-1}$ (s. Tab. 4.3) und ist damit weit niedriger als die Vergleichswerte aus Naturwäldern. Es muss davon ausgegangen werden, dass in den Jahren 1990 bis 1997, in der das Gebiet der Bundesforstverwaltung unterstellt war, noch einmal eine verstärkte Nutzung stattgefunden hat (mündl. Mitteilung FUCHS, ehemaliger Revierförster). Die vereinzelt auffindbaren Stubben deuten ebenfalls darauf hin.

Auch Mund (2004) ermittelte für nahe gelegene Buchenbestände im Nationalpark geringe Totholzmengen, was sie auf eine frühere Nutzung des Bestände zurück führt. Die Werte von totem Derbholz schwanken ebenfalls stark zwischen ca. 6.8 und $57.8 \mathrm{~m}^{3} \mathrm{ha}^{-1}$. Das von der Autorin gewählte Aufnahmeverfahren zählt Totholz ab einer Stärke von $5 \mathrm{~cm}$ Durchmesser zum Derbholz. In der vorliegenden Studie wurden erst Stamm- und Astfragmente ab einem Durchmesser von $7 \mathrm{~cm}$ aufgenommen. Dies führt zu etwas niedrigeren Werten, die damit nur bedingt vergleichbar sind.

Der im Vergleich zu den anderen Flächen relativ hohe Totholzanteil auf Fläche 2 setzt sich zum größten Teil aus einigen in jüngster Zeit abgestorbenen Buchen und Eichen zusammen (vgl. Abb. 4.6). Die auf dieser Fläche dominierende Winterlinde trägt auch einen hohen Anteil zum Gesamtbetrag bei, jedoch sind die Totholzmengen über alle Zerfallsklassen verteilt, was einer kontinuierlichen Mortalitätsrate entspricht. Der überproportionale Anteil von abgestorbenen Buchen und Eichen bedeutet einen Rückgang dieser ohnehin auf der Fläche gering vertretenen Arten (Buche: 12.06\%, Eiche: 17.29\% der Grundfläche). MUND (2004) fand in den untersuchten Buchenbeständen mit geringer Beimischung anderer Arten $(10 \%$ und 38\%) einen mit 66, bzw. 48\% überdurchschnittlich hohen Anteil derselben am Totholzaufkommen, woraus sie eine kontinuierliche Entmischung des Buchenwaldes prognostiziert. Lässt man sich auf diese Spekulation ein, kann man in dem großen Anteil an Buchentotholz im von Winterlinden dominierten Lindig ebenfalls einen Prozess der Entmischung sehen, jedoch ist es hier die Buche, die anteilmäßig zurückgeht.

$\mathrm{Zu}$ einer Einschätzung der künftigen Bestandesentwicklung ist es jedoch notwendig, nicht nur die Verjüngung und die Totholzvorkommen als Kriterien heran zu ziehen, sondern auch das Verhalten der Altbäume im Bestand unter Berücksichtigung der Nachbarschaftsinteraktion, worauf in den folgenden Kapiteln eingegangen wird. 


\subsection{Artspezifische Kronenarchitektur und räumliche Interaktion zwischen Altbäumen von Rotbuche, Esche, Hainbuche und Winterlinde}

\subsubsection{Artspezifische Unterschiede der Kronenform}

Die untersuchten Baumarten unterscheiden sich in ihren Kronendimensionen in charakteristischer Weise und lassen unterschiedliche Strategien der horizontalen und vertikalen Kronenraumerschließung erkennen.

Bei der Auswertung der Kronenprojektionsflächen zeigte sich, dass die zusätzliche Messung der sonnenexponierten Kronenfläche (ECA) den erheblichen Mehraufwand bei der Datenerhebung durchaus rechtfertigt. Sie liefert die wichtige Zusatzinformation, in welchem Verhältnis Sonnen- und Schattenkronen-Anteile zueinander stehen. Für die Produktivität eines Baumes, die eng an die Effizienz der Lichtabsorption der Blätter gebunden ist, ist die Sonnenkrone sehr viel wichtiger als der Schattenkronen-Bereich (HOLBROOK \& LUND 1995). Die signifikant größeren Kronenflächen der Hainbuchen im Vergleich zu Winterlinde und Esche auf allen vier Flächen, an die nur die Buche partiell heranreicht (s. Abb. 4.7), sind auf eine sehr viel stärker ausgeprägte Schattenkrone zurückzuführen. Die Flächengrößen der Sonnenkronen unterschieden sich dagegen nicht signifikant voneinander. Wie HAGEMEIER (2002) belegt, investiert die Hainbuche im Vergleich zu Buche und Winterlinde deutlich mehr Kohlenstoff in die Bildung einer ausgeprägten Schattenkrone. Diese dient vermutlich eher der effizienten Beschattung des Waldbodens, um die Etablierung weniger schattentoleranter Arten zu verhindern, als der Kohlenstoff-Fixierung (HAGEMEIER 2002; LEUSCHNER 1998). Da diese für die Hainbuche mit vergleichsweise hohen Kosten verbunden ist (HAGEMEIER 2002), sind die großen Kronenflächen nicht als Faktor zu bewerten, der eine Überlegenheit in der Raumeroberung und Lichtnutzung anzeigt. Die Effizienz der Schattenbildung ist bei der Hainbuche nicht nur durch eine große Kronenfläche gegeben. Sie verfügt mit einem artspezifischen Blattflächenindex (CAI) von 5.47 auch über die größte Belaubungsdichte (vgl. Abb. 4.8).

Die untersuchten Winterlinden wiesen ebenfalls eine hohe Belaubungsdichte auf (CAI= 4.89). Der prozentuale Anteil der Schattenkrone - also der nicht direkt sonnenexponierten Kronenanteile - lag weit unter dem der Hainbuchen (32\%), jedoch wies diese Art auf allen Flächen auch die kleinsten Sonnenkronen auf $\left(28.71 \mathrm{~m}^{2}-39.60 \mathrm{~m}^{2}\right)$. Auf drei der vier Flächen wies die Buche signifikant größere Sonnenkronen auf $\left(46.10 \mathrm{~m}^{2}-75.22 \mathrm{~m}^{2}\right)$. 
Das fast vollständige Fehlen einer Schattenkrone ist charakteristisch für die Esche, die sich im Laufe ihres Lebens von einer schattentoleranten Art in eine Lichtbaumart verwandelt (LEIBUNDGUT 1991, MARIGO et al. 2000). Bis zum Erreichen der Bestandeshöhe bildet sie nur sehr kleine, kurze Kronen aus und zeigt unter guten Lichtbedingungen (ab einer relativen Beleuchtungsstärke von 10\%) ein schnelles Höhenwachstum, worin sie in diesem Stadium die Buche noch übertrifft (MARIGO et al. 2000). Erst in der obersten Kronenschicht zeigt sich eine verstärkte laterale Ausbreitung sowohl des Stammes als auch der Krone. Daraus ergibt sich der enge, zu Beginn exponentielle Zusammenhang zwischen Stammdurchmesser und Kronenausdehnung (s. Abb. 4.9). Einzelne Eschen auf den Untersuchungsflächen erreichten einen BHD von über $80 \mathrm{~cm}$. Diese waren im Kern hohl und wiesen eine, vermutlich altersbedingt, geringere Vitalität auf. Diese Individuen hatten zwar mit $125-212 \mathrm{~m}^{2}$ sehr große Kronen, das BHD/Kronenflächen-Verhältnis lag jedoch unter dem Durchschnitt der jüngeren, in der Optimalphase befindlichen Eschen. Das bewirkt ein Abflachen der Kurve im oberen BHD-Bereich, woraus sich ein hochsignifikanter sigmoidaler Kurvenverlauf für die Korrelation des Stammdurchmessers mit der Kronenfläche ergibt $\left(\mathrm{r}^{2}=0.88, \mathrm{p}<0.00001\right)$.

Auf den untersuchten Flächen befanden sich keine Linden mit einem BHD von über $65 \mathrm{~cm}$. So kann nicht nachvollzogen werden, ob sich der dargestellte exponentielle Kurvenverlauf, der das Verhältnis von BHD und Kronenausdehnung für die Winterlinde am besten beschreibt $\left(\mathrm{r}^{2}=0.65, \mathrm{p}<0.00001\right)$, bei Altbäumen mit einem BHD von über $80 \mathrm{~cm}$ wieder abflachen würde. Dies ist anzunehmen, da Laubbäume die laterale Ausdehnung im Kronenraum mit zunehmender Seneszenz einschränken (ROLOFF 2001).

Die Strategie, zunächst verstärkt in die Höhe zu wachsen und erst mit Erreichen der obersten Kronenschicht in die laterale Ausdehnung zu investieren, teilen Eschen und Winterlinden. Bei den untersuchten Buchen scheint sie sehr viel weniger ausgeprägt und bei den aufgenommenen Hainbuchen ist sie nicht erkennbar. Bei dieser Art fanden sich einzelne Individuen, die schon mit einem BHD von $20-25 \mathrm{~cm}$ Kronenflächen von über $100 \mathrm{~m}^{2}$ aufwiesen. Für beide Arten lies sich das BHD/Kronenflächen-Verhältnis am besten mit einer linearen Gleichung beschreiben. Aufgrund der starken Streuung der Werte erklärt das Modell jedoch nur 42\% der Gesamtvarianz bei der Buche und nur 27\% bei der Hainbuche.

Um zu prüfen, welche anderen Bestandesparameter eine Auswirkung auf die Größe der Kronenfläche haben könnten, wurde für alle vier Baumarten eine multiple lineare Regressionsanalyse durchgeführt (s. Tab. 5.1). Diese bezieht neben den individuellen Größen BHD und Kronenlänge auch Parameter mit ein, die die Konkurrenzsituation der Einzelbäume beschreiben. 
Tab 5.1: Multiple lineare Regression der verschiedenen Einflussfaktoren auf die artspezifische Kronenfläche mit schrittweisem Ausschluss nicht signifikanter Parameter $(\mathrm{p} \leq 0.05)$. In das Modell gehen folgende Parameter als unabhängige Variablen ein: Stammdurchmesser (dbh), Kronenlänge (crown length), Distanz zum nächsten Nachbarn mit gleichem oder größerem BHD (dist. to dom. NB), Stammdurchmesser des nächsten Nachbarn mit gleichem oder größerem BHD (dbh dom. NB), Untersuchungsfläche (plot) und ein Konkurrenzindex (CI), der die Konkurrenzsituation des individuellen Baumes im Kronenraum beschreibt (nähere Erläuterung im Text).

\begin{tabular}{llccccc}
\hline Species & Dependent Variable & $\mathbf{n}$ & $\mathbf{r}^{2}{ }_{\text {adj. }}$ & Std. Error & $\mathbf{F}$ & Sig. \\
\hline Fagus & crown area & 115 & 0.55 & 21.15 & 29.07 & $<0.0001$ \\
sylvatica & & & & & & \\
\cline { 2 - 7 } & Independent Variables & Std. Error & $\mathbf{t}$ & Sig. & Excluded Variables \\
\cline { 2 - 6 } & dbh & 0.24 & 5.65 & $<0.0001$ & dist. to dom. NB \\
& crown length & 2.87 & 3.64 & $<0.0001$ & dbh dom. NB \\
Cl & 8.53 & 1.98 & 0.05 & & \\
plot & 2.27 & -1.98 & 0.05 & &
\end{tabular}

\begin{tabular}{llccccr}
\hline Species & Dependent Variable & $\mathbf{n}$ & $\mathbf{r}^{2}{ }_{\text {adj. }}$ & Std. Error & $\mathbf{F}$ & \multicolumn{1}{c}{ Sig. } \\
\hline $\begin{array}{l}\text { Fraxinus } \\
\text { excelsior }\end{array}$ & crown area & 135 & 0.81 & 18.32 & 196.18 & $<0.0001$ \\
\cline { 2 - 7 } & & & & & \\
\cline { 2 - 7 } & Independent Variables & Std. Error & $\mathbf{t}$ & Sig. & Excluded Variables \\
\cline { 2 - 6 } & 0.12 & 17.63 & $<0.0001$ & & plot \\
& (Constant) & 8.24 & -6.57 & $<0.0001$ & & crown length \\
& dist. to dom. NB & 0.73 & 3.14 & 0.002 & & dbh dom. NB \\
Cl & 6.63 & 2.85 & 0.005 & & \\
\hline
\end{tabular}

\begin{tabular}{llccccr}
\hline Species & Dependent Variable & $\mathbf{n}$ & $\mathbf{r}^{2}$ adj. & Std. Error & $\mathbf{F}$ & Sig. \\
\hline $\begin{array}{l}\text { Carpinus } \\
\text { betulus }\end{array}$ & crown area & 56 & 0.51 & 23.33 & 13.22 & $<0.0001$ \\
\cline { 2 - 7 } & & & & & \\
\cline { 2 - 7 } & Independent Variables & Std. Error & $\mathbf{t}$ & Sig. & Excluded Variables \\
& Cl & 18.70 & 4.30 & $<0.001$ & & crown length \\
& plot & 3.61 & -3.58 & 0.001 & & \\
dbh & 0.50 & 3.51 & 0.001 & & \\
(Constant) & 36.70 & -3.04 & 0.004 & & \\
& dbh dom. NB & 1.79 & 2.63 & 0.01 & & \\
dist. to dom. NB & 0.40 & 2.68 & 0.01 & & \\
\hline
\end{tabular}

\begin{tabular}{llccccr}
\hline Species & Dependent Variable & $\mathbf{n}$ & $\mathbf{r}^{2}$ adj. & Std. Error & $\mathbf{F}$ & \multicolumn{1}{c}{ Sig. } \\
\hline Tilia & crown area & 230 & 0.70 & 15.33 & 181.51 & $<0.0001$ \\
cordata & & & & & & \\
\cline { 2 - 7 } & Independent Variables & Std. Error & $\mathbf{t}$ & $\mathbf{S i g .}$ & Excluded Variables \\
\cline { 2 - 6 } & dbh & 0.14 & 14.94 & $<0.0001$ & & plot \\
& (Constant) & 7.13 & -11.31 & $<0.0001$ & crown length \\
& Cl & 4.91 & 8.32 & $<0.0001$ & dist. to dom. NB \\
& dbh dom. NB & 0.28 & 2.50 & 0.01 & & \\
\hline
\end{tabular}


Dazu gehören zwei einfache Kenngrößen der Konkurrenzsituation, aus denen HEGYI (1974) einen Konkurrenzindex entwickelte, der sich bis heute gegen später entwickelte Indices behauptet (Lorimer 1983, PiutTi \& Cescatti 1997, Bachmann 2002, Shi \& Zhang 2003): die Distanz zum nächsten Nachbarbaum, der einen gleichen oder größeren BHD besitzt sowie der BHD dieses Nachbarn. Zusätzlich wurde ein Index (CI) berechnet, der die spezifische Konkurrenzsituation im Kronenraum beschreibt. Er wurde von DöBBELER et al. (2003) in Anlehnung an Wensel et al. (1987) entwickelt. Dieser Index-Wert eines Einzelbaumes ist die Summe der Kronenschirmflächen aller benachbarten Bäume, die in einer Höhe von $66 \%$ der Kronenlänge des Bezugsbaumes geschnitten werden. Liegt der Kronenansatz eines Nachbarbaumes über der Schnitthöhe, so wird seine volle Kronenschirmfläche berücksichtigt. Ist der Baum kleiner als die Schnitthöhe, wird er nicht berücksichtigt. So lässt sich dieser Wert als Kronenschlussgrad auf einer Höhe von zwei Dritteln der Kronenlänge interpretieren. Auf dieser Höhe liegt für die Buche der Übergang zwischen Licht- und Schattenkrone (GUERICKE 2001).

Als nominaler Parameter fließt die jeweilige Untersuchungsfläche mit ein, um eventuelle Unterschiede innerhalb einer Baumart zwischen den Flächen nachzuweisen. Auf weitere Wuchsparameter wie zum Beispiel die Baumhöhe wurde verzichtet, da diese stark mit dem BHD und der Kronenlänge korreliert ist und so nicht als unabhängige Variable angesehen werden kann.

Die Kronenflächen der Buchen werden im Modell zum größten Teil aus baumeigenen Wuchsparametern erklärt. Brusthöhendurchmesser und Kronenlänge weisen mit 5.65 bzw. 3.64 die größten t-Werte auf. Doch wie schon die verhältnismäßig schwache Beziehung zwischen BHD und Kronenfläche dieser Art (s. Abb. 4.9, $\mathrm{r}_{\text {adj. }}^{2}=0.46$ ), so ist auch bei dieser Regression der $\mathrm{r}^{2}$ adj. Wert mit 0.55 nicht hoch. Doch durch die hinzugenommenen Parameter konnte der erklärte Anteil an der Gesamtvarianz etwas erhöht werden. So hat der Kronenschlussgrad einen signifikanten Einfluss auf die Kronengröße. Auch zeigt das Modell eine Abnahme der Kronengröße von Fläche 1 hin zu Fläche 4, was auch in Abb. 4.7 deutlich wird und sich aus der unterschiedlichen Bestandesstruktur und -geschichte erklärt (s. Kap. 5.1.1). Die Distanz zum nächsten dominanten Nachbarn und dessen Stammstärke haben im Modell keinen Einfluss auf die Kronengröße.

Durch die sehr viel engere Beziehung zwischen BHD und Kronenfläche bei der Esche ist auch der erklärte Anteil an der Gesamtvarianz deutlich höher als bei der Buche $\left(\mathrm{r}^{2}{ }_{\text {adj. }}=0.81\right)$. Der BHD hat den weitaus stärksten Einfluss auf das Modell, weitere Einflussgrößen sind der Kronenschlussgrad und die Distanz zum nächsten dominanten Nachbarn. 
Sehr ähnliche Verhältnisse der Einflussgrößen und ein hoher Korrelationskoeffizient $\left(r^{2}{ }_{\text {adj. }}=0.70\right)$ zeigen sich bei den untersuchten Winterlinden. Auch hier spielt der Einfluss des BHD die weitaus größte Rolle. Der Kronenschlussgrad ist noch stärker gewichtet und der BHD des nächsten dominanten Nachbarbaums geht in das Modell mit ein. In beiden Fällen zeigen sich keine Unterschiede zwischen den Untersuchungsflächen.

Die Hainbuche ist die einzige Art, bei der der Stammdurchmesser nicht der stärkste Einflussfaktor ist. Die Kronenlänge als zweiter baumeigener Parameter fließt als einziger nicht mit in das Modell ein, alle äußeren, Konkurrenzeinflüsse anzeigenden Parameter haben hingegen einen signifikanten Einfluss. Der Kronenschlussgrad hat mit 4.30 den höchsten tWert, aber auch Distanz und Dimension des nächsten größeren Baumes sind wichtige Parameter. Wie bei der Buche zeigt sich auch ein Flächeneinfluss, der sich aus den sehr hohen Kronenflächen auf Fläche 1 erklärt.

Damit zeigt sich die Hainbuche in ihrer Kronenausdehnung deutlich als am stärksten von Nachbarn beeinflusste Art.

Tab 5.2: Multiple lineare Regression der verschiedenen Einflussfaktoren auf die artspezifische Fläche der Sonnenkrone mit schrittweisem Ausschluss nicht signifikanter Parameter $(\mathrm{p}<0.05)$. In das Modell gehen folgende Parameter als unabhängige Variablen ein: Stammdurchmesser (dbh), Kronenlänge (crown length), Distanz zum nächsten Nachbarn mit gleichem oder größerem BHD (dist. to dom. NB), Stammdurchmesser des nächsten Nachbarn mit gleichem oder größerem BHD (dbh dom.NB), Untersuchungsfläche (plot) und ein Konkurrenzindex (CI), der die Konkurrenzsituation des individuellen Baumes im Kronenraum beschreibt (nähere Erläuterung im Text).

\begin{tabular}{llccccr}
\hline Species & Dependent Variable & $\mathbf{n}$ & $\mathbf{r}^{{ }^{2} \text { adj. }}$ & Std. Error & $\mathbf{F}$ & \multicolumn{1}{c}{ Sig. } \\
\hline Fagus & Exposed crown area & 115 & 0.64 & 16.20 & 53.94 & $<0.0001$ \\
sylvatica & & & & & & \\
\cline { 2 - 7 } & Independent Variables & Std. Error & $\mathbf{t}$ & Sig. & Excluded Variables \\
\cline { 2 - 6 } & dbh & 0.17 & 8.03 & $<0.0001$ & & Cl \\
& (Constant) & 7.28 & -3.42 & 0.001 & & plot \\
& crown length & 0.54 & 3.05 & 0.003 & & \\
& dist. to dom. NB & 0.81 & 2.12 & 0.03 & & \\
& dbh dom. NB & 0.13 & -2.54 & 0.013 & & \\
\hline
\end{tabular}

\begin{tabular}{llccccr}
\hline Species & Dependent Variable & $\mathbf{n}$ & $\mathbf{r}^{2}$ adj. & Std. Error & $\mathbf{F}$ & Sig. \\
\hline $\begin{array}{l}\text { Carpinus } \\
\text { betulus }\end{array}$ & Exposed crown area & 56 & 0.51 & 22.61 & 21.43 & $<0.0001$ \\
\cline { 2 - 7 } & & & & & & \\
\cline { 2 - 7 } & Independent Variables & Std. Error & $\mathbf{t}$ & Sig. & Excluded Variables \\
& dbh & 0.43 & 5.56 & $<0.001$ & & crown length \\
& (Constant) & 28.67 & -3.31 & 0.002 & plot \\
& dist. to dom. NB & 1.68 & 2.67 & 0.01 & & dbh dom. NB
\end{tabular}


Auf Grund der großen Schattenkronen-Anteile an der gesamten Kronenfläche bei Buche und Hainbuche wurde für diese Baumarten die gleiche Regressionsanalyse nur für die Sonnenkrone durchgeführt (s. Tab 5.2). Für die Buche verbessert sich der Erklärungswert des Modells deutlich, wenn nur die Sonnenkrone berücksichtigt wird $\left(\mathrm{r}^{2}{ }_{\text {adj. }}=0.64\right)$, für die Hainbuche bleibt er gleich. Bei beiden Arten gibt es keinen Unterschied mehr zwischen den Untersuchungsflächen und auch der Kronenschlussgrad hat keinen signifikanten Einfluss mehr auf das Modell. Für die Buche verstärkt sich allerdings der distanz- und dimensionsabhängige Einfluss der Nachbarbäume. Die Hainbuche hingegen zeigt sich in der Expansion der Lichtkrone weniger von Kronenkonkurrenz beeinflusst. Die Distanz zum nächsten Nachbarbaum ist die einzige signifikante Einflussgröße aus der Gruppe der exogenen Faktoren. Der Brusthöhendurchmesser hat nun auch für diese Baumart den höchsten Erklärungswert.

Diese Werte lassen die Vermutung zu, dass die Hainbuche mit dem Ausbilden einer großen Schattenkrone sich bereits so viel Standraum erobert, dass sich die Sonnenkrone weniger stark von Konkurrenz beeinflusst proportional zum Dickenwachstum des Stammes ausbreiten kann.

\subsubsection{Nachbarschaftsinteraktion im Kronenraum}

Im geschlossenen Bestand wird der Wuchsraum wesentlich von den Pflanzen geprägt, die in unmittelbarer Nachbarschaft wachsen, da diese, je nach artspezifischen Ansprüchen und Abstand, mehr oder weniger intensiv um die gleichen Ressourcen konkurrieren (OLIVER et al. 1990). Die Konkurrenzintensität zwischen benachbarten Pflanzen nimmt im Allgemeinen, bei geringer Störung, mit der Qualität des Nährstoffangebots eines Standortes zu (GRIME 1979; FRELICH et al. 1998). Unter diesen Bedingungen treten die Pflanzen vor allem in Wettbewerb um die Ressource Licht, die - anders als die relativ gleichmäßig im Boden verteilten Nährstoffe - als Strahlung von oben einfällt und damit direktional gerichtet erscheint. So ergeben sich für Pflanzen, die auch nur einen geringen Vorsprung im Höhenwachstum aufweisen, erhebliche Vorteile (GRACE 1990), was zu stark asymmetrischen Konkurrenzsituationen führt (SCHWINNING \& WEINER 1997).

Für die Beurteilung der gemessenen Kronenüberlappungen ist es demnach wichtig, ein dreidimensionales Bild des Kronenraumes zu entwickeln, um differenzieren zu können, welcher Baum den anderen überschirmt und welcher überschirmt wird. Dazu dient der zusätzlich zur Kronenausdehnung erhobene Parameter der Höhe der maximalen Ausdehnung (s. Abb. 4.10). Mit diesem ist es möglich, ein Kronenmodell $\mathrm{zu}$ entwerfen und die Unterschiedlichkeit der Baumarten in ihrer Kronenausdehnung nicht nur auf horizontaler, 
sondern auch auf vertikaler Ebene darzustellen. In Abbildung 5.2 sind die dafür relevanten Parameter Baumhöhe, Kronenansatzhöhe, Kronenradius und Höhe der maximalen Ausdehnung als Mittelwerte und Standardabweichung aufgetragen und durch ein Kronenmodell nach PRETzSCH \& SEIFERT (2000) zusammengefasst. Die baumartenspezifische Anpassung für Esche, Hainbuche und Winterlinde erfolgte nach DöBBELER et al. (2003).

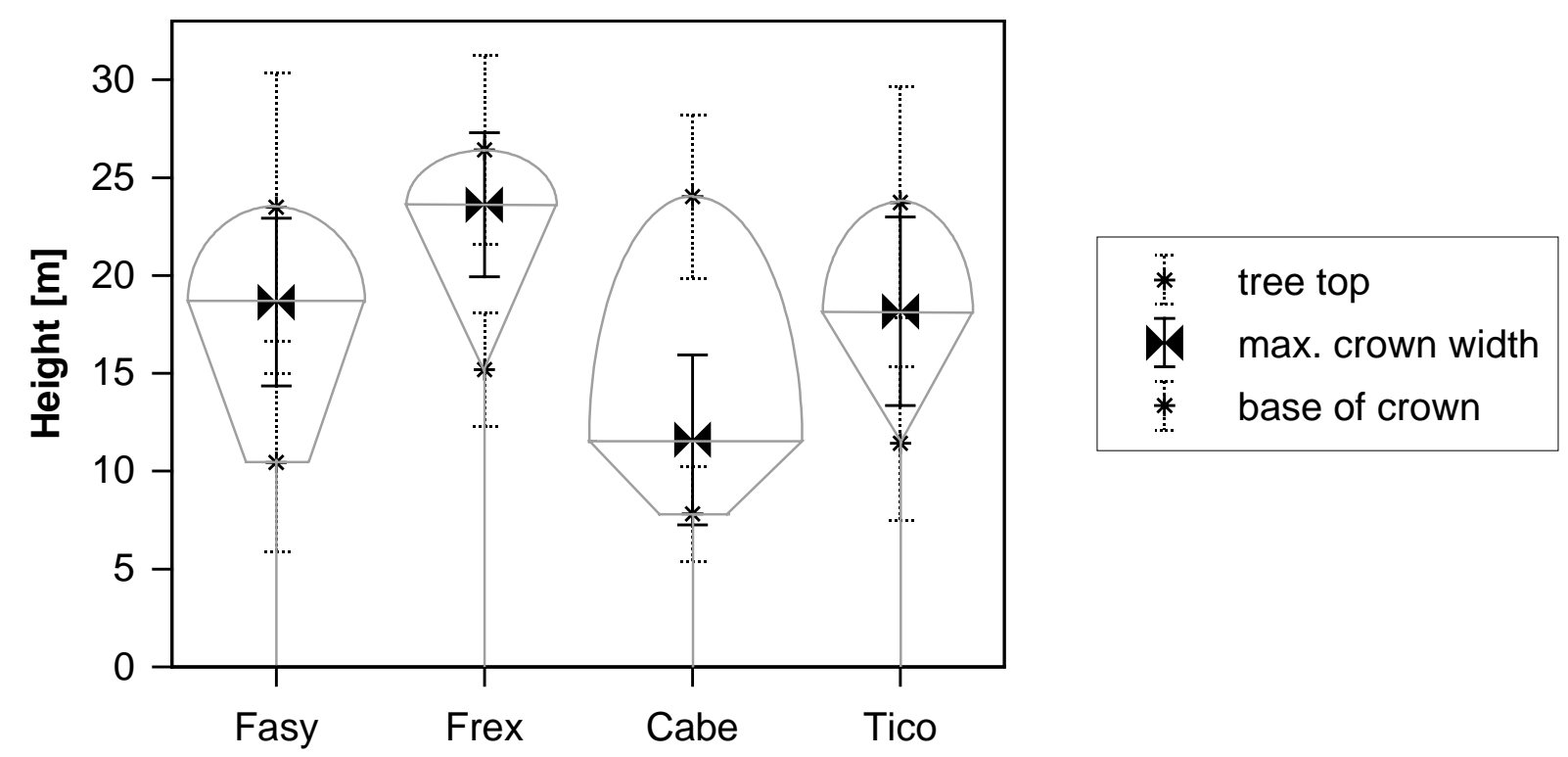

Abb. 5.2: Artspezifische Kronenform, die sich aus den Parametern Kronenansatz (base of crown), Baumhöhe (tree top), Höhe der maximalen Ausdehnung (max. crown width) und dem mittleren Kronenradius ergibt.

In dieser maßstabsgetreuen Gegenüberstellung zeigt sich, wie sich die unterschiedliche Kronenarchitektur der Baumarten auf die Interaktion im Kronenraum auswirkt. Die Esche überschirmt ihre Nachbarn auch bei gleichen Oberhöhen deutlich, da sie Ihre Kronen auf einem signifikant höheren Niveau von 21 bis $27 \mathrm{~m}$ ausbreitet. Die Hainbuche ist in allen Fällen die überschirmte Baumart.

Die höchsten Überlappungen im Kronenbereich wurden für benachbarte Eschen und Hainbuchen ermittelt (s. Abb. 4.11). Die starke vertikale Aufteilung der Kronenraumes erlaubt es diesen Arten, in unmittelbarer Nachbarschaft auch bei ähnlicher Stammstärke einen zweischichtigen Kronenraum zu bilden, bei dem die Hainbuche durchschnittlich 29\%, in Extremfällen aber auch bis zu 50\% der Kronenfläche benachbarter Eschen mit einnimmt (s. Abb. 4.12). Die Bildung eines mehrschichtigen Kronenaufbaus im temperaten Laubmischwald bei gleich alten Bäumen mit unterschiedlicher Kronenarchitektur und Lichtansprüchen beschreibt SCHÜTZ (1994) als günstige Baumartenmischung. Aufgrund der höheren Biomasse- 
Akkumulation sind sie von großem ökologischen Interesse. Solche Bestände sind dann produktiver als Reinbestände, wenn die unterständige Art die unproduktive Schattenkrone der überschirmenden Art ersetzt (WIERMANN \& Oliver 1979, SCHÜTZ 1989, KELTY 1989).

Die Zweischichtigkeit des Kronenaufbaus zwischen benachbarten Eschen und Hainbuchen scheint langfristig stabil zu sein und sich mit fortschreitendem Höhenwachstum noch zu verstärken. Mit zunehmender Kronenlänge, die für beide Baumarten eng mit dem Höhenwachstum korreliert ist (Tab. 5.3, linke Hälfte), steigt für Eschen auch die Höhe der maximalen Ausdehnung (Tab. 5.3, rechte Hälfte). Hainbuchen senken das Höhenniveau der maximalen Kronenbreite mit zunehmender Kronenlänge tendenziell ab (r=-0.26).

Tab. 5.3: Lineare Regression (lineare Gleichung der Form $y=a+b x$ ) der Kronenlänge mit der Baumhöhe und der Höhe der maximalen Kronenbreite.

\begin{tabular}{lccccccccc}
\hline Species & \multicolumn{4}{c}{ Crown length / Hight } & \multicolumn{5}{c}{ Max. Crown width / Crown length } \\
\cline { 2 - 10 } & $\mathrm{n}$ & $\mathrm{a}$ & $\mathrm{b}$ & $\mathrm{r}$ & $\mathrm{p}$ & $\mathrm{a}$ & $\mathrm{b}$ & $\mathrm{r}$ & $\mathrm{p}$ \\
\cline { 2 - 10 } F. sylvatica & 113 & 3.24 & 0.40 & 0.41 & $<0.001$ & 20.7 & -0.15 & -0.11 & 0.123 \\
F. excelsior & 104 & -8.83 & 0.76 & 0.75 & $<0.001$ & 18.92 & 0.36 & 0.41 & $<0.0001$ \\
C. betulus & 57 & -3.45 & 0.83 & 0.76 & $<0.001$ & 15.81 & -0.26 & -0.26 & 0.024 \\
T. cordata & 147 & 0.16 & 0.52 & 0.46 & $<0.001$ & 19.65 & -0.11 & -0.06 & 0.202 \\
\hline
\end{tabular}

Für Buche und Winterlinde zeigt sich kein Zusammenhang zwischen Kronenlänge und Höhe der maximaler Kronenbreite. Auch die Beziehung zwischen Höhe und Kronenlänge ist sehr viel schwächer ausgeprägt. Beide Arten konkurrieren um genau den gleichen Kronenraum, da sie ihre Kronen auf dem gleichen Höhenniveau entfalten, ohne eine Stratifizierungstendenz mit zunehmender Höhe zu zeigen.

Die Hainbuche weist auch in Nachbarschaft $\mathrm{zu}$ diesen Arten relativ große Überlappungsbereiche auf. Alle interspezifischen Nachbarschaften, an denen die Hainbuche nicht beteiligt ist, weisen signifikant niedrigere Überlappungswerte auf. Dies liegt einerseits an den sehr viel geringeren Kronenflächen der anderen drei Baumarten, andererseits aber auch an der weniger starken vertikalen Aufteilung des Kronenraumes. Esche und Winterlinde zeigen in interspezifischen Kombinationen stets größere Überlappungsbereiche als in intraspezifischen, was DEGENHARDT (1994) für die Esche ebenfalls nachweist. Für die Buche ist dies nicht der Fall. In Nachbarschaft zu Linden und Eschen sind die Überlappungen geringer; im Falle der Eschen-Nachbarschaft ist dieser Unterschied signifikant. Diese 
Beobachtung deckt sich nicht mit den Untersuchungen von PRETZSCH (1992) an Fichten- und Buchenkronen, der eine sehr viel stärkere Kronenüberlappung beider Arten in Mischung als im Reinbestand fand. Zu größeren Überlappungen als mit gleichartigen Nachbarn kommt es bei der Buche nur in Nachbarschaft zu Hainbuchen. Letztere weisen eine deutlich andere Kronenstruktur auf, die insofern mit der Fichte vergleichbar ist, als dass sie im unteren Kronendrittel am breitsten ist und nach oben hin schmaler wird. Für eine Bewertung des Verhältnisses von intraspezifischer und interspezifischer Kroneninteraktion ist es somit notwendig, die jeweils aufeinander treffenden artspezifischen Kronenstrukturen $\mathrm{zu}$ berücksichtigen (SZWAGRZYK 1997, PRETZSCH 2002). Für die im letzten Jahrzehnt verstärkt und mit zunehmendem Erfolg entwickelten Waldwachstumsmodelle (Überblicksdarstellungen dazu liefern z. B. Bossel 1991, Pretzsch 2000, Pacala \& CANHam 2002, Vester 2002 und GATZER 2004) fehlt bislang eine ausreichende empirische Grundlage zur Modellierung der unterschiedlichen Kronenformen von Hainbuche, Esche und Winterlinde. Die hier vorgestellten Ergebnisse zeigen, dass eine Zusammenfassung dieser Arten und eine einheitliche Schätzung der Parameter Kronenlänge und Höhe der maximalen Ausladung aus BHD und Höhe wichtige strukturelle Unterschiede unterschlägt, die für die Beurteilung der Kompatibilität dieser Arten und einer Prognose der Bestandesentwicklung wichtig sind. 


\subsection{Mechanismen der Kronenraumeroberung}

Die Erforschung der Baumkronen als komplexer Lebensraum hat vor allem durch die Entwicklung sicherer und praktikabler Zugangstechniken in den vergangenen zwei Jahrzehnten große Fortschritte erzielt (NADKARNI 2001), die die unterschiedlichsten Forschungsrichtungen der Botanik Zoologie und Klimatologie etc. mit einbezieht (LOWMAN \& WiTTMAN 1996). Trotzdem wurden Untersuchungen zur Kroneninteraktion zwischen Gehölzpflanzen bislang meist an Jungbäumen (Z.B. FrANCO 1986; KoIKE 1989; KIKUZAWA 1996; OzAWA et al. 2000, GRACE \& Platt 2002) oder Hecken (KÜPPERS 1985, 1987) durchgeführt. STERCK \& BONGERS (2001) zeigen jedoch in Modellierungsversuchen, dass Jungpflanzen für die Vorhersage des vegetativen Wachstums als Reaktion auf Licht nicht als Modellorganismen für die Kronenentwicklung in Altbäumen herangezogen werden können. Auch die Allometrie des Trieblängenwachstums ändert sich mit zunehmender Baumhöhe (OSADA et al. 2002) und Astordnung (BUCK-SORLIN \& BELL 2000).

Die wichtigsten Ausgangshypothesen für die Interaktion von Baumkronen sind i) dass die verschiedenen Arten sich auf morphologischer Ebene deutlich unterscheiden und die entstehenden Strukturen auf einer unterschiedlichen Kronenarchitektur beruhen (z.B. OLIVER et al. 1990; YoKOZAWA et al. 1996, VESTER 1997) und ii) dass die Konkurrenz um Licht den stärksten Einfluss auf die Ausbildung der individuellen Kronenform hat. Die gegenseitige Beschattung wird als die wichtigste Form der Interaktion im Kronenraum gesehen (z.B. KoIKe 1986; OzAWA et al. 2000, TREMMEL \& BAZZAZ 1993).

Eine weitere Form der Kroneninteraktion ist der direkte Kontakt benachbarter Kronen bei Wind, der zu mechanischen Schädigungen führen kann. Diese Interaktionsform wurde bislang relativ wenig untersucht, obwohl sie erhebliche Auswirkungen auf die Kronenentwicklung haben kann (RUDNICKI et al. 2001). Studien zu diesem Phänomen beschränken sich meist auf Untersuchungen auf Bestandesebene (FLESCH \& WILSON 1999) oder betrachten extreme Windereignisse, um die Sturmfestigkeit forstwirtschaftlich wichtiger Arten zu charakterisieren (z.B. MOORE \& MAGUIRE 2005). Studien zur Frage, inwiefern Kronenkollisionen das Trieblängenwachstum einzelner Äste im Kronenraum von Altbäumen beeinflussen, fehlen bislang.

FRANCO (1986) fasst ganz allgemein die möglichen Interaktionsformen benachbarter Baumkronen sehr prägnant zusammen, wobei er drei Formen der Interaktion unterscheidet, wenn zwei Triebe benachbarter Kronen aufeinandertreffen: 1. Beide Triebe stellen ihr Wachstum ein oder ändern die Orientierung. 2. Ein Trieb weicht aus oder stellt sein 
Wachstum ein, während der andere weiter wächst. 3. Keiner der Triebe lässt sich durch die Anwesenheit des anderen am Wachstum hindern. Daraus resultieren folgende Wuchsformen: 1. Klare Separation der benachbarten Triebe (,shyness'). 2. Ein einseitiges DominanzVerhältnis, das bei Bäumen $\mathrm{zu}$ Kronenasymmetrie führt. 3. Gegenseitige physische Schädigung beider Nachbarn durch Zweigabbrüche. Dieses Phänomen kann optisch ebenfalls den Eindruck von ,shyness' hervorrufen.

Die fehlenden Untersuchungen an Altbäumen sind auf die methodische Schwierigkeit zurückzuführen, einen störungsfreien Zugang in die Baumkrone zu erlangen. Durch die besondere Möglichkeit des Kronenzugangs mit einer mobilen Hebebühne konnte dieses Hindernis in der vorliegenden Studie überwunden werden. Es wurde ein methodisches Konzept zur Untersuchung der Kroneninteraktion entwickelt, bei dem insgesamt 682 Äste an 42 Individuen untersucht wurden, das eine Auswertung auf Astebene wie auf Individuenebene ermöglicht.

\subsubsection{Baumartenspezifische Merkmale auf Astebene}

In den hier vorgestellten ersten Auswertungsschritten zeigen sich zunächst die artspezifischen Unterschiede deutlicher als die Unterschiede in der Reaktion auf die einzelnen Nachbararten. So zeigte sich an den untersuchten Eschenzweigen, dass diese Art bei ähnlichem Längenwachstum sehr viel dickere Zweige ausbildete, die sich kaum verzweigten, an deren Ende jedoch ein sehr dichtes Blattbüschel $\mathrm{zu}$ finden war. Wie auch schon an anderen Parametern der Bestandesstruktur (z.B. Baumhöhe, Höhe der maximalen Ausdehnung) zu sehen war, zeigten die Hainbuchen ein konträres Verhalten. Buchen und Winterlinden wiesen hingegen bei allen untersuchten Parametern sehr ähnliche Werte auf, was auf eine hohe Konkurrenzintensität zwischen beiden Arten hinweist. Lediglich in der Anzahl der Kurztriebe unterschieden sich Buchen und Winterlinden erheblich. Die Buche wies 4.5 mal so viele Kurztriebe wie die Winterlinde auf, die im Durchschnitt nur jedes dritte Jahr einen Kurztrieb ausbildete. Diese weniger als $1.5 \mathrm{~cm}$ langen Seitenzweige werden von der Buche in hoher Zahl ausgebildet und dienen als Überdauerungsorgane, die zumeist nur apikal 3-5 Blätter ausbilden. Die lateralen Knospen sind ausschließlich ,schlafende’ Proventivknopsen, die nicht austreiben. Somit verzweigen sich Kurztriebe nicht und bilden mit der Zeit sogenannte Kurztriebketten (ROLOFF 2001). Die Proventivknospen bleiben als Reserveknospen über viele Jahre erhalten und können jederzeit austreiben, sobald sich die lokalen Lichtverhältnisse ändern (RolofF 1988). Damit hat die Buche ein sehr großes Potenzial, schnell in neu 
entstandene Kronenlücken einzuwachsen. In einem so dicht geschlossenen Bestand wie dem hier untersuchten, kann dieses Potenzial ein entscheidender Konkurrenzvorteil sein.

\subsubsection{Beschattung und Kronenkollision als Formen der Kroneninteraktion}

Eine denkbare Form der Kroneninteraktion wäre eine frühzeitige Reaktion auf eine durch Überschirmung veränderte Lichtqualität. Höhere Pflanzen sind mit Hilfe des Photorezeptors Phytochrom B in der Lage, den Anteil an Infrarot-Strahlung an der Gesamtstrahlung zu bestimmen. Damit können sie Bereiche meiden, die von Nachbarpflanzen bereits überschattet werden. Bislang ist ein aktives Umgehen von Bereichen mit erhöhtem Infrarotanteil und damit geringerer Lichtqualität nur bei krautigen Pflanzen beobachtet worden (z.B. BARNES et al. 1990, BALlaré et al. 1995, Aphalo et al. 1999). Muth \& BAZZAZ (2002) konnten diesen Effekt bei Baumsämlingen (Betula papyrifera Marshall) nicht nachweisen. Auch Buchen- und Eichen-Jungpflanzen zeigten nach AMMER (2003) in Beschattungsexperimenten keine spezifische Reaktion auf ein verändertes Rot/Infrarot-Verhältnis an der Gesamtstrahlung. In beiden Versuchen war die Licht-Quantität entscheidend, nicht die Qualität.

Die Messungen der relativen Beleuchtungsstärke an den Triebspitzen der untersuchten Zweige zeigen, dass das Längenwachstum nicht allein mit dem Lichtgenuss erklärt werden kann (Tab. 4.4). Lediglich für die Buchen zeigt sich ein signifikanter Einfluss der Lichtintensität auf die Trieblänge. Da sich schon der quantitative Einfluss der gesamten photosynthetisch aktiven Strahlung nur für eine von vier Baumarten statistisch absichern ließ, schien eine Reaktion auf eine veränderte Lichtqualität unwahrscheinlich. Diese ebenfalls denkbare Form der Kroneninteraktion wurde nicht gesondert untersucht.

Eine mögliche Erklärung für den fehlenden Zusammenhang zwischen Längenwachstum und Beleuchtungsstärke bietet die hohe Anzahl an Leittriebunterbrechungen. Da alle Baumarten an mehr als 50\% aller Äste mindestens einen Abbruch der Haupttriebachse aufwiesen (Abb. 4.17), muss angenommen werden, dass die tatsächlich erbrachte Zuwachsleistung deutlich höher lag. Vor allem aber zeigen die hohen Abbruchraten, dass eine wesentliche Form der Interaktion zwischen benachbarten Bäumen die durch Wind induzierte Kronenkollision darstellt. Schon bei leichtem Wind kollidieren die Baumkronen in der obersten Bestandesschicht und fügen sich bei stärkerem Wind deutlich sichtbare, zum Teil erhebliche Verletzungen zu. Dabei spielen die Unterschiede in der Triebmorphologie eine wichtige Rolle. So sind die dünnen Zweige der Hainbuche bei Kollisionen mit Nachbarkronen stärker betroffen als die der anderen Arten. Vor allem in Nachbarschaft zu Eschen mit ihren dicken, starren, unverzweigten Trieben waren bei dieser Art jeweils mehrere Abbrüche pro Zweig zu 
beobachten. Die Esche hatte im seitlichen Kronenbereich durch ihre Triebmorphologie einen wesentlichen Vorteil. Im Wipfelbereich erwiesen sich ihre starren Zweige mit dem dichten Blattbüschel am Ende jedoch als sehr windanfällig und brachen auch ohne Einwirkung der Nachbarkronen sehr leicht. So sind die im Wipfelbereich mehr als 5 mal so hohen Abbruchraten dieser Art im Vergleich zu den anderen Baumarten zu erklären. Die häufigen Astabbrüche der Esche gleichen optisch den bei der Eiche durch Astreinigung zu Boden fallenden Zweigfragmenten. Bei Eschen gibt es jedoch keine aktive Abszission wie bei Eichen (RUST \& ROLOFF 2004).

Mit jedem Astabbruch verliert der Baum Biomasse und darüber hinaus auch den von diesem Zweig eingenommenen Raum in der Baumkrone. Da im Nachhinein nicht feststellbar ist, wie viele Zuwachseinheiten bei einer Kronenkollision abbrechen, konnte dieser Verlust nicht quantifiziert werden. Aus den vorgenommenen Astwinkelmessungen ließ sich jedoch berechnen, wie gut die untersuchten Baumarten die durch Abbrüche zunächst verursachte Änderung der Wuchsrichtung kompensieren und den verlorenen Raum wiederbesiedeln können. Tatsächlich sind die Abweichungen des Astwachstums durch abbruchbedingte Winkeländerungen von einer idealen Geraden nicht sehr hoch. Im seitlichen Kronenbereich waren die Strecken der aufsummierten Trieblängen zwischen 5 und 15\% länger als die berechnete Vektor als kürzeste Verbindungsstrecke zwischen Astansatz und Triebspitze. Die Differenzen zwischen aufsummierten Trieblängen und dem Vektor im seitlichen Kronenbereich waren jedoch bei Buche, Esche und Winterlinde signifikant größer als in den deutlich gerader gewachsenen Wipfeltrieben (s. Abb. 4.18). Hainbuche und Winterlinde zeigten insgesamt die stärksten Richtungsänderungen. In intraspezifischen Nachbarschaftskonstellationen waren diese signifikant größer als zwischen artgleichen Paaren von Buche und Esche. In interspezifischen Konstellationen zeigten die jeweils direkt benachbarten Arten keine signifikanten Unterschiede in der relativen Astlänge. Eine Ausnahme bildeten benachbarte Buchen- und Lindenzweige. Letztere wiesen im Extrem eine Abweichung von 55\% in Richtung angrenzender Buchen auf. Bei den Messungen im Freiland wurde auch beobachtet, dass Lindentriebe nach mehrfachen Abbrüchen in manchen Fällen die Wuchsrichtung grundlegend änderten und wieder in die eigene Krone zurückwuchsen.

Modellierungsansätze, die das Wuchsverhalten von Altbäumen im geschlossenen Bestand simulieren, nehmen für das Astwachstum eine gerade Linie an (z.B. GRAMS et al. 2002), bzw. gehen von einem strikt radialen Wachstum im seitlichen Kronenbereich aus (GROTE \& PRETZSCH 2002). Aufgrund der vorliegenden Daten müsste eine artspezifische Abweichung 
von dieser Geraden im seitlichen Kronenbereich mit einbezogen werden, die für Buche und Esche bei 5\% und für Hainbuche und Winterlinde bei 10\% liegt.

\subsubsection{Gewichtung der Einflussgrößen auf das Trieblängenwachstum}

Um den Einfluss der verschiedenen erhobenen Parameter auf das Trieblängenwachstum zu gewichten, wurde eine multiple lineare Regression für alle vier Baumarten durchgeführt (s. Tab. 5.4).

Die in das Modell einfließenden Parameter lassen sich in drei Gruppen einteilen: Die erste Gruppe bilden baumartspezifische Parameter, die die Kronenarchitektur miteinbeziehen, wie die Position innerhalb der Baumkrone (Wipfel oder seitlicher Kronenbereich), die Astordnung, Astumfang und vorhandene oder nicht vorhandene Fruchtbildung am untersuchten Triebabschnitt. Die zweite Gruppe setzt sich aus abiotischen Faktoren zusammen, die die räumliche und zeitliche Stellung der untersuchten Triebe berücksichtigen. Dazu gehören die Exposition (diese wurde zur statistischen Auswertung in ,northerness' und ,easterness' umgewandelt), das Messjahr und die Untersuchungsfläche. Zur dritten Gruppe gehören Faktoren, die auf Nachbarschaftseffekte zurückgehen. Hierzu gehört die relative Beleuchtungsstärke (PAR), die Abbruchrate und die Artzugehörigkeit des Nachbarbaumes.

Der Erklärungswert des Modells ist für die Buche mit $r_{\text {adj. }}{ }^{2}=0.74$ deutlich höher als für die anderen drei Baumarten (Esche: $r_{\text {adj. }}{ }^{2}=0.43$, Hainbuche: $r_{\text {adj. }}{ }^{2}=0.51$, Winterlinde: $r_{\text {adj. }}{ }^{2}=0.48$ erklärt). Bei allen vier untersuchten Baumarten üben Faktoren der ersten Gruppe den größten Einfluss aus. Für die Buche ist es die Stellung des Triebes in der Krone, für alle übrigen Baumarten ist der Durchmesser des jeweiligen Astes der wichtigste Parameter. Für Esche und Hainbuche ist darüber hinaus auch die Astordnung von Bedeutung, obwohl nur die Triebspitzen mit hoher Astordnung untersucht wurden (Ordnung 1-5), um diesen Effekt zu minimieren. Diese deutliche Abhängigkeit des Wachstums einzelner Triebe von der internen Kronenarchitektur wurde von HALLÉ et al. (1978) erstmals detailliert qualitativ beschrieben. Sie steht im Gegensatz zu der von HARPER (1981) formulierten Vorstellung von Ästen als autonomen Modulen, die unabhängig voneinander auf die lokalen Bedingungen reagieren. Diese Vorstellung liegt vielen Wachstumsmodellen zu Grunde (z.B. SORRENSEN-COTHERN et al. 1993). Gegen Zweigautonomie sprechen auch die Experimente von HENRIKSSON (2001) an Birke und von STOLL \& SCHMID (1998) an Kiefer. Die einzelnen Äste hatten eine signifikant unterschiedliche Überlebensrate, je nachdem, ob der ganze Baum beschattet wurde oder nur Teilbereiche. 
Tab. 5.4: Multiple lineare Regression der verschiedenen Einflussfaktoren auf das artspezifische Trieblängenwachstum unter schrittweisem Ausschluss nicht signifikanter Parameter $(p<0.05)$. In das Modell gehen folgende Parameter als unabhängige Variablen ein: Fläche (plot), Messjahr (year), relative Beleuchtungsstärke (PAR), Position in der Krone (position in canopy), Astumfang (perimeter), Abbruchrate (breakages), Exposition (northerness/easterness), Fruktifikation (fructification), Astordung (branch order) und Artzugehörigkeit des Nachbarbaumes (species NB).

\begin{tabular}{llccccr}
\hline Species & \multicolumn{1}{c}{ Dependent Variable } & $\mathbf{n}$ & $\mathbf{r}^{2}{ }_{\text {adj. }}$ & Std. Error & $\mathbf{F}$ & \multicolumn{1}{c}{ Sig. } \\
\hline Fagus & shoot length & 144 & 0.74 & 5.72 & 67.35 & $<0.0001$ \\
\cline { 2 - 7 } sylvatica & Independent Variables & Std. Error & $\mathbf{t}$ & $\mathbf{S i g}$ & Excluded Variables \\
\cline { 2 - 7 } & position in canopy & 0.21 & 5.74 & $<0.0001$ & & northerness \\
& perimeter & 0.53 & 4.72 & $<0.0001$ & & easterness \\
& plot & 1.03 & -3.50 & $<0.0001$ & & fructification \\
& PAR & 0.03 & 2.23 & 0.03 & & branch order \\
& breakages & 3.91 & -2.13 & 0.03 & & species NB \\
& year & 0.94 & 2.06 & 0.04 & & \\
constant & 1889.27 & -2.06 & 0.04 & & \\
\hline
\end{tabular}

\begin{tabular}{clccccr}
\hline Species & Dependent Variable & $\mathbf{n}$ & $\mathbf{r}^{2}{ }_{\text {adj. }}$ & Std. Error & $\mathbf{F}$ & \multicolumn{1}{c}{ Sig. } \\
\hline Fraxinus & shoot length & 141 & 0.43 & 7.39 & 22.11 & $<0.0001$ \\
\cline { 2 - 7 } excelsior & Independent Variables & Std. Error & $\mathbf{t}$ & $\mathbf{S i g .}$ & Excluded Variables \\
\cline { 2 - 7 } & perimeter & 0.69 & 5.01 & $<0.0001$ & & northerness \\
& constant & 5.68 & -4.08 & $<0.0001$ & easterness \\
plot & 1.39 & 3.64 & $<0.0001$ & fructification \\
branch order & 0.65 & 2.53 & 0.01 & breakages \\
position in canopy & 0.25 & 2.11 & 0.04 & & year \\
& & & & PAR \\
& & & & & species NB
\end{tabular}

\begin{tabular}{clccccr}
\hline Species & Dependent Variable & $\mathbf{n}$ & $\mathbf{r}^{2}{ }_{\text {adj. }}$ & Std. Error & $\mathbf{F}$ & \multicolumn{1}{c}{ Sig. } \\
\hline Carpinus & shoot length & 138 & 0.51 & 4.43 & 18.75 & $<0.0001$ \\
\cline { 2 - 7 } betulus & Independent Variables & Std. Error & $\mathbf{t}$ & $\mathbf{S i g .}$ & Excluded Variables \\
\cline { 2 - 7 } & perimeter & 0.36 & 4.78 & $<0.0001$ & & fructification \\
& position in canopy & 0.21 & 4.25 & $<0.0001$ & & year \\
& branch order & 0.40 & 3.91 & $<0.0001$ & & PAR \\
& species NB & 0.43 & -3.90 & $<0.0001$ & & northerness \\
& plot & 1.13 & -3.49 & $<0.0001$ & & easterness \\
& breakages & 2.22 & -2.70 & 0.01 & & \\
& constant & 2.90 & 1.38 & $<0.0001$ & & Sig. \\
\hline Species & Dependent Variable & $\mathbf{n}$ & $\mathbf{r}^{2}{ }_{\text {adj. }}$ & Std. Error & $\mathbf{F}$ & $<0.0001$ \\
\hline Tilia & shoot length & 592 & 0.48 & 7.84 & 23.76 & Excluded Variables \\
\cline { 2 - 7 } cordata & Independent Variables & Std. Error & $\mathbf{t}$ & Sig. & position in canopy \\
& perimeter & 0.54 & 6.92 & $<0.0001$ & branch order \\
& fructification & 1.94 & -2.88 & $<0.0001$ & & breakages \\
& constant & 3239.85 & -2.33 & 0.02 & & northerness \\
& year & 1.62 & 2.33 & 0.02 & & easterness \\
& species NB & 0.72 & -2.05 & 0.04 & & PAR \\
& & & & & plot \\
& & & & &
\end{tabular}


Einige neuere Modellierungsversuche beziehen die Gebundenheit des Triebwachstums an die Kronenposition mit ein (z.B. BUCK-SORLIN \& BELL 2000, NiKINMAA et al. 2003).

Fruktifikation als weiterer bauminterner Parameter wirkt sich nur bei Winterlinden signifikant auf das Triebwachstum aus. Das hängt mit der terminalen Stellung des Blütenstands am Zweig zusammen (GLEISSNER 1998), wodurch das Längenwachstum eingeschränkt ist. Bei Eschen sind die Blüten- und Fruchtstände basal und determinieren somit das Längenwachstum nicht. Hainbuchen weisen ebenfalls apikale Fruchtstände auf. Die untersuchten Individuen trugen in den Untersuchungsjahren im Gegensatz zur Winterlinde wenige Früchte, so dass der Anteil an fruchttragenden Zweigen sehr gering war und somit in der statistischen Auswertung nicht ins Gewicht fiel.

In der Gruppe der räumlichen und zeitlichen Faktoren zeigen sich bei Buche, Esche und Hainbuche Unterschiede im Trieblängenwachstum zwischen den Untersuchungsflächen. Dieser Faktor ging als nominaler Faktor in das Modell ein. Buchen und Hainbuchen weisen auf Fläche 1 größere Zuwächse auf, Eschen zeigen auf Fläche 2 größeres Wachstum. Diese Unterschiede lassen sich mit den Dimensionsunterschieden der Individuen erklären. Buchen und Hainbuchen weisen signifikant größere Kronenflächen auf Fläche 1 auf (s. Abb. 4.7) und dominieren daher im Kronenbereich. Eschen zeigen auf Fläche 2 ein stärkeres Wachstum, was darauf hindeutet, dass es keine durchgehend besseren Wachstumsbedingungen auf Fläche 1 gibt. Bei Winterlinden zeigt sich kein Einfluss der Untersuchungsfläche. Für diese Art bestehen jedoch, wie für die Buche, Unterschiede im Wachstum zwischen den Untersuchungsjahren 2003 und 2004, was mit der Trockenheit im Jahr 2003 zusammenhängen könnte.

Das Trieblängenwachstum ist bei allen Arten unabhängig von der Himmelsrichtung. Die Äste an der Nordseite eines Baumes sind also nicht prinzipiell kürzer. Damit sind auch eventuelle Kronenasymmetrien nicht von der Richtung des Strahlungseinfalles abhängig, sondern allein auf unterschiedliche Nachbarschaftsbedingungen zurückführbar. $\mathrm{Zu}$ diesem Ergebnis kamen auch FRECH et al. (2002) im gleichen Bestand bei einer Analyse der Kronenasymmetrien auf der Grundlage von Berechnungen des Mittelpunktes der Kronenprojektionsflächen im Verhältnis zum Stammfußpunkt. Der Einfluss der Exposition verstärkt sich erst weiter nördlich, wie ROUVINEN \& KUULUVAINEN (1997) an Kiefernkronen in Ostfinnland nördlich des $62^{\circ}$ Breitengrades feststellten.

Die in der dritten Gruppe zusammengefassten Nachbarschaftsparameter haben auf die vier Baumarten einen sehr unterschiedlich starken Einfluss. Für das Triebwachstum von Eschen 
erwies sich keiner der Parameter als relevant. Hainbuchenzweige werden am stärksten negativ von der Abbruchrate beeinflusst. Auch die Artzugehörigkeit des Nachbarbaumes hat einen signifikanten Einfluss. Dieser zeigt sich auch bei der Winterlinde.

Der relative Lichtgenuss an der Triebspitze hat auch in der multifaktoriellen Analyse nur bei der Buche einen signifikanten Einfluss auf die Trieblänge. Dieser geringe Einfluss wird eventuell noch durch die methodische Schwierigkeit verringert, dass die Lichtbedingungen über der Triebspitze nicht unbedingt mit den Lichtbedingungen im Vorjahr am gleichen Trieb vergleichbar sind, also mit dem Zeitpunkt der Knospenanlage für den vermessenen Triebabschnitt. Es kann aber davon ausgegangen werden, dass sich die Beleuchtungsverhältnisse innerhalb eines Jahres im geschlossenen Kronendach nicht wesentlich ändern. Genauere Untersuchungen dazu wurden aber bislang nicht durchgeführt.

Die Sensitivität des Zweigwachstums auf negative Nachbarschaftseffekte steigt demnach von der Esche über die Winterlinde zur Buche und schließlich zur Hainbuche hin an.

\subsubsection{Interaktion auf Individuenebene - die Kontaktzone}

Die Auswirkungen der unterschiedlichen Triebmorphologie und Kronenarchitektur zeigen sich noch deutlicher auf der nächsthöheren Skalenebene bei der Untersuchung der Dimensionen der Kontaktzonen zwischen den einzelnen Baumarten. Die Esche hatte in allen Nachbarschaftskonstellationen extrem kurze beblätterte Kontakt-Zonen (Abb. 4.19), die sich stets nur über 15 - 20\% der gesamten gemeinsamen Kronenlänge erstreckten. In den darunter gelegenen Bereichen waren immer nur Blätter von einem Nachbarbaum zu finden. Hier haben die Verdrängungs- und Ausweichprozesse zu einer deutlichen Aufteilung des Kronenraums geführt. Die Konkurrenzintensität steigt also mit dem Höhenniveau im Bestand deutlich an und ist im obersten Bereich, wenige Meter unter den Wipfeln, am stärksten. Dies gilt für alle Artkombinationen. Winterlinden und Hainbuchen bilden dabei aufgrund ihrer feinen, dicht verzweigten Triebe und ihrer langen Kronen signifikant längere Kontakt-Zonen untereinander aus als in Nachbarschaft zu Buchen oder Eschen.

Da bislang solche Untersuchungen in temperaten Mischwäldern noch nicht durchgeführt wurden und somit keine Möglichkeit eines Datenvergleichs bestand, wurde eine Kooperation mit der Universität Leipzig aufgebaut. Mit dem gleichen methodischen Vorgehen wurden Kontaktzonen im Leipziger Auwald mit Hilfe eines Krans im Zuge des Leipziger-AuwaldKranprojektes (LAK) vermessen (TAL 2006). Der dortige Bestand setzt sich hauptsächlich aus Fraxinus excelsior, Acer pseudoplatanus, Tilia cordata, Carpinus betulus, und Quercus robur zusammen und weist von der BHD-Verteilung her eine sehr heterogene Struktur auf, sodass 
TAL (2006) den Bestand in einzelne, patches' von einheitlicher Struktur unterteilt. Durchmesser, Kronenflächen und Standraumbedingungen der im Hainich untersuchten Bäume sind vergleichbar mit dem patch-Typ 2 bei TAL (2006). In Tabelle 5.5 ist die vertikale Erstreckung der Kontaktzonen der in beiden Beständen untersuchten Artpaare vergleichend gegenübergestellt. Die Längen der Kontaktzonen von Eschen zu artgleichen Nachbarn, aber auch zu Winterlinden und Hainbuchen liegen in beiden Beständen zwischen 2 und 3 Metern. Auch der prozentuale Anteil der Interaktionszone an der gesamten Kronenlänge liegt im Auwald wie im Hainich zwischen 15\% und 20\%. Die Kontaktzonen zwischen Hainbuchen und Winterlinden sind im Hainich jedoch deutlich länger. In beiden Beständen unterscheiden sie sich jedoch signifikant von den Artkombinationen, an denen die Esche beteiligt ist. Winterlinden in artgleicher Nachbarschaft weisen im Hainich die längsten Kontaktzonen auf. Das ist im Leipziger Auwald nicht der Fall.

Tab. 5.5: Länge der Kontaktzonen sowie deren relativer Anteil an der Kronenlänge der jeweils beteiligten Arten für die Untersuchungsstandorte Leipziger Auwald und Hainich. Kleinbuchstaben kennzeichnen Unterschiede zwischen den Artpaaren innerhalb eines Bestandes (Wilkoxon U-Test, $\mathrm{p}<0.05$ ). Daten für den Leipziger Auwald nach TAL (2006) und pers. Mitteilung)

\begin{tabular}{|c|c|c|c|c|}
\hline \multirow{2}{*}{$\begin{array}{c}\text { Species } \\
\text { combination } \\
\text { Art A-Art B }\end{array}$} & \multicolumn{2}{|c|}{$\begin{array}{l}\text { Length of contact zones } \\
{[\mathrm{m}]}\end{array}$} & \multicolumn{2}{|c|}{\begin{tabular}{|c} 
Percentage of crown length \\
{$[\%]$}
\end{tabular}} \\
\hline & Leipzig & Hainich & $\begin{array}{c}\text { Leipzig } \\
\text { Art A-Art B }\end{array}$ & $\begin{array}{c}\text { Hainich } \\
\text { Art A-Art B }\end{array}$ \\
\hline Frex-Frex & $2.6 \mathrm{ab}$ & $1.9 \mathrm{a}$ & 20 & 15.4 \\
\hline Frex-Tico & $2.5 \mathrm{a}$ & $3.0 \mathrm{a}$ & $18.3-16.7$ & $19.9-20.8$ \\
\hline Frex-Cabe & $2.7 \mathrm{a}$ & $2.2 \mathrm{a}$ & $17.8-21.8$ & $17.9-12.6$ \\
\hline Cabe-Tico & $4.4 \mathrm{~b}$ & $7.3 \mathrm{~b}$ & n.d. -24 & $37.7-47.2$ \\
\hline Tico-Tico & $3.2 \mathrm{ab}$ & $8.4 \mathrm{~b}$ & 20 & 52.6 \\
\hline
\end{tabular}

TAL (2006) zeigt durch seine Unterteilung der untersuchten Baumpaare in drei BHD-Klassen, dass die Länge der Kontaktzone mit zunehmender Stammstärke und Kronenfläche nicht zunimmt, sondern konstant bei $15-20 \%$ der Kronenlänge liegt. Der direkte Kronenkontakt zwischen Altbäumen findet auch in späteren Entwicklungsstadien nur in den ersten Metern unterhalb des Wipfels statt. Jedoch nimmt aufgrund der sich vergrößernden horizontalen Kronenausdehnung die Breite des Kontaktbereichs signifikant zu, die in dieser Untersuchung nicht mit aufgenommen wurde. 
Eine weitere Zusatzuntersuchung des Vertikalprofils von toten Ästen und Zweigen zeigt, dass das höchste Totholzvolumen in der Krone in den vier Metern direkt unterhalb der beblätterten Kontaktzone liegt (TAL 2006). Demnach schließt sich an den Kontaktbereich, in dem die lebenden Organe der benachbarten Bäume aufeinandertreffen, eine Kontaktzone abgestorbener Kronenbestandteile an. Dieses Phänomen konnte im Hainich ebenfalls beobachtet werden. Besonders abgestorbene, aber noch nicht abgeworfene Starkäste können das Einwachsen junger Bäume von untern erheblich beeinträchtigen und benachbarten Kronen, vor allem beblätterten Zweigen, durch Kollision massive Schäden zufügen. Die Astwinkeländerungen und Abbruchraten lebender Zweige, die auf benachbarte tote Kronenbereiche stoßen, sind deutlich größer als die innerhalb der beidseitig beblätterten Kontaktzone. Da diese Untersuchung auf die Frage der Mechanismen der Kronenraumeroberung ausgerichtet war, schien eine Beschränkung auf die beblätterte gemeinsame Kontaktzone sinnvoll, in der auch noch aktives Ausweichen von beiden Seiten aus möglich ist. Weitere Untersuchungen, die auch den Erhalt des einmal eingenommenen Kronenraums mit einschließen, müssen diesen Kontaktbereich unterhalb der beblätterten Zone in jedem Fall miteinbeziehen.

Neben der vertikalen Ausdehnung wurde die Intensität des Kronenkontaktes zwischen Nachbarbäumen klassifiziert (s. Tab. 4.5). Diese Einteilung macht es möglich, die von FRANCO (1986) aufgestellten Hypothesen zu den möglichen Formen der Kroneninteraktion zu überprüfen und die Kontaktzonen der untersuchten Altbäume nach diesem Schema zu ordnen. Die erste Form, die Franco als ,shyness' bezeichnet und durch aktives Umlenken der Wuchsrichtung, bzw. Einstellen des Wachstums zustande kommt, noch bevor die benachbarten Individuen tatsächlich aufeinander stoßen, konnte bei den untersuchten Baumkronen nicht gefunden werden. Diese Interaktionsform ist schon theoretisch in einem geschlossenen Bestand nahezu ausgeschlossen, da bei einer Bestandeshöhe von $30 \mathrm{~m}$ Kronenkollisionen nicht $\mathrm{zu}$ vermeiden sind. Schon bei schwachem Wind berühren sich benachbarte Kronen. Die Lichtverhältnisse ändern sich mit jeder Kronenbewegung, so dass ein Detektieren der Lichtqualität für die Pflanze obsolet erscheint. Die zweite Form ist die einseitige Dominanz, die dadurch entsteht, dass einer der angrenzenden Triebe sein Wachstum einstellt und der andere den gesamten Raum einnimmt. Dies konnte nur in geringem Maße beobachtet werden. Die nachbarschaftsbezogene Auswertung der Trieblängen zeigt kaum einseitige Dominanz einer Art gegenüber einer anderen im seitlichen Wipfelbereich (s. Abb. 4.13). Zwar sind die Längenzuwächse der Hainbuchentriebe im Mittel etwas geringer als bei den anderen Arten, spezifische Einschränkungen einer Art in 
Nachbarschaft zu einer andern waren jedoch nicht signifikant. Die mit Abstand häufigste und die Kronenform prägende Art der Interaktion ist die dritte von Franco (1986) genannte Möglichkeit: Beide Triebe wachsen aufeinander zu, bis es zu mechanischer Schädigung kommt. Davon sind zunächst beide Nachbarn betroffen. Wie stark die Schädigung durch Kollision für den Trieb ist, hängt jedoch stark von dessen morphologischen Eigenschaften ab. Hier hat die Esche mit ihren signifikant dickeren, wenig verzweigten Trieben einen entscheidenden Vorteil gegenüber den anderen Arten und besonders gegenüber der Hainbuche. Wie von Franco vorhergesagt, kommt es auch bei dieser Interaktionsform zu einer Separation der Kronen, die optisch dem Shyness-Effekt gleicht. Dieses Phänomen, bei dem die benachbarten Kronen sich bei Windstille nicht berühren, konnte für fast alle ArtKombinationen, an denen die Esche beteiligt ist, nachgewiesen werden. Es zeigte sich auch bei $80 \%$ aller Hainbuchen in artgleichen Paaren.

Eine von Franco in seine theoretischen Überlegungen nicht mit einbezogene Möglichkeit ist das Einwachsen eines Triebes in die Sphäre der Nachbarpflanze, bzw. das gemeinsame Besiedeln des Zwischenraums. Die häufigste Form der Kronennachbarschaft im untersuchten Bestand war ein Ineinandergreifen der Zweige über eine Strecke von bis zu $50 \mathrm{~cm}$. Vor allem Buchen untereinander und in Nachbarschaft zu Hainbuchen zeigten in rund 75\% aller Fälle dieses Verhalten. Die Winterlinde zeigte darüber hinaus verstärkt die Tendenz, auch tiefer in die Kronen benachbarter Bäume einzudringen. Bei benachbarten Hainbuchen wurden bei $60 \%$ und in Nachbarschaft zu Eschen bei $80 \%$ aller untersuchten Kontaktzonen ein Ineinandergreifen von mehr als $50 \mathrm{~cm}$ gemessen. Offenbar sind die Endtriebe der Winterlinde so flexibel, dass sie durch den mechanischen Kontakt nicht immer abbrechen. Diese Form des gegenseitigen Eindringens in die Krone des Nachbarbaums ist für Hecken nicht nachgewiesen (KÜPPERS 1987) und wurde auch bei Quercus-Arten nicht gefunden (KOIKE 1989). Im Leipziger Auwald konnte dieses Phänomen bei Winterlinden und Eschen jedoch auch beobachtet werden (pers. Mitteilung O. TAL). Hier zeigen sich große artspezifische Unterschiede, die bislang nicht untersucht wurden.

Das Fehlen dieser Möglichkeit des Ineinandergreifens benachbarter Kronen in den theoretischen Überlegungen von FRANCO (1986) und die weit verbreitete Vorstellung von klar abgegrenzten Einflusszonen von Einzelbäumen (BELLA 1971), die bis heute die Grundlage für einzelbaumbasierte Modellierungen ist (z.B. SHI \& ZHANG 2003, GROTE \& PRETZSCH 2002), zeigt jedoch auch, dass es nicht nur an empirischen Untersuchungen zur Kronenstruktur mangelt, sondern dass auch die theoretischen Grundlagen der Kronenforschung hinter der Komplexität dieses immer noch wenig verstandenen Lebensraumes zurückbleiben. 


\subsection{Dendroökologische Untersuchungen}

\subsubsection{Stammzuwachs und Trieblängenwachstum}

Bislang wurden wenig Studien durchgeführt, die das Verhältnis von Trieblängenwachstum und Stammzuwachs untersuchen (WorBES et al. 1992). Dies liegt zum Teil daran, dass Trieblängenrückmessungen nicht wie die Jahrringbreiten über das ganze Baumleben zurückverfolgt werden können, zum Teil aber auch an der unterschiedlichen Methodik. Auch in dieser Arbeit mussten für die Auswahl der Probebäume für die dendroökologischen Untersuchungen einerseits und die Trieblängenmessungen andererseits unterschiedliche Kriterien angewendet werden. Für die Zuwachsbohrungen war es wichtig, vorherrschende oder herrschende Bäume jeder Art auf jeder Fläche auszuwählen, bei den Trieblängenmessungen war die direkte Nachbarschaft aller Artenkombinationen ausschlaggebend. Dennoch konnten für jede Art sechs - bei der Winterlinde fünf - Individuen mit beiden Methoden untersucht werden.

Nach WENZEL (1987) ist der Höhenzuwachs von Bäumen ein empfindlicher Indikator für wechselnde Umweltbedingungen, der spontaner und nachhaltiger reagiert als das Dickenwachstum. Darum wurden Untersuchungen zum Höhenzuwachs auch verstärkt in den 1980er Jahren im Zuge der Waldschadensdiskussion durchgeführt. Diese konzentrierten sich jedoch vor allem auf die Fichte (PfeILSTRICKER 1983, APPT et al. 1983, KenNEL 1984, SCHMidT \& HARTMANN 1984, Worbes et al. 1995). Einige Studien gibt es auch zur Buche (z. B. RolOFF 1985, BRAUN \& FLÜCKIGER 1987). BRAUN \& FLÜCKIGER (1987) beobachteten als Reaktion auf das Trockenjahr 1976 eine starke Reduktion des Höchenzuwachses von Buchen in der Nordwestschweiz und dem Kanton Zürich im Folgejahr 1977.

Ein ähnliches Phänomen zeigte sich bei den untersuchten Buchen im Hainich für das Trockenjahr 2003. Im selben Jahr sind die Höhenzuwächse überdurchschnittlich gut (vgl. Abb. 4.20, Kap. 4.4.1), um im Jahr 2004 drastisch abzufallen. Auch das Dickenwachstum geht 2004 zurück, die Unterschiede sind jedoch nicht so stark ausgeprägt.

Für die untersuchten Buchen ließ sich das Höhenwachstum der Wipfeltriebe und das Dickenwachstum des Stammes nicht korrelieren (vgl. Tab. 4.7, Kap. 4.4.1). Lediglich das über Gipfel- und Seitentriebe gemittelte Wachstum wies für zwei der sechs untersuchten Individuen eine signifikant positive Beziehung zum Stammzuwachs auf. WORBES et al. (1992) fanden hingegen an Buchen an einem flachgründigen Südhang im Weserbergland eine signifikante, jedoch breit gestreute Korrelation zwischen den Jahrringbreiten und den Trieblängen das Vorjahres. Der Zusammenhang wird mit Verweis auf RoLOFF (1985) damit 
erklärt, dass durch das gebundene Wachstum der Triebe, bei dem die Knospenanlage eines Triebes bereits im Vorjahr erfolgt, die klimatischen Bedingungen des Vorjahres ausschlaggebend für das Wachstum sind. An den im Hanich untersuchten Bäumen konnte wie auch von BRAUN \& FLÜCKINGER (1987) kein Zusammenhang des Triebwachstums mit den Stammzuwächsen, bzw. dem Klima des Vorjahres festgestellt werden.

Wie die Autoren selbst vermuten, ist der von WORBES et al. (1992) aufgezeigte starke Zusammenhang vor allem durch die sehr trockenen standörtlichen Bedingungen zu erklären.

Die untersuchten Buchen wiesen ein durchschnittliches Dickenwachstum von nur $1 \mathrm{~mm}$ auf und erreichten Höhen von bis zu $13 \mathrm{~m}$.

Der direkte Zusammenhang von Höhen- und Dickenzuwachs ist auch darum erstaunlich, da die Hauptanteile von Streckungswachstum und Dickenwachstum zu verschiedenen Jahreszeiten erfolgen (RAUH 1939, LEBOURGEOIS et al. 2005). Dies zeigte sich sehr gut an einer der untersuchten Eschen und an einer Winterlinde. Eine Korrelation beider Parameter kam für diese Arten nur dann zu einem signifikanten Ergebnis, wenn das Jahr 1999 nicht berücksichtigt wurde (s. Tab. 4.7; Kap. 4.4.1). In diesem Jahr war der Juli extrem niederschlagsreich und gleichzeitig auch sehr warm. Unter diesen Bedingungen bildeten die Arten extrem viel Spätholz im Vergleich zu den fünf Jahren davor und danach. Im Juli war das Wachstum der Zweige jedoch schon vollständig abgeschlossen, sodass eine direkte Reaktion auf positive Witterungsverhältnisse nicht möglich war. Im Folgejahr 2000 stieg das Trieblängenwachstum bei Esche und Winterlinde an, jedoch war die Zunahme nicht signifikant und insgesamt geringer als der Wachstumsschub des Stammes im Vorjahr.

\subsubsection{Einfluss des Klimas auf die Zuwachsdynamik}

Die Auswahl von möglichst gut vergleichbaren Probebäumen wurde durch die unterschiedlichen Artenzusammensetzungen und Häufigkeitsverteilungen auf den vier Untersuchungsflächen erschwert. So waren auf den Flächen 1 und 2 fast ausschließlich sehr alte Buchen mit großem BHD zu finden, die in ihrer Vitalität teilweise schon eingeschränkt waren. Beim Anbohren zeigte sich, dass der Kern schon teilweise zersetzt oder mit Wasser gefüllt war, sodass eine Jahrringanalyse nicht möglich war. Andere Bäume wiesen so geringe Zuwächse auf, dass keine Gleichläufigkeit mit der Mittelkurve festgestellt werden konnte. So mussten von den ursprünglich 20 ausgewählten Individuen 5 verworfen werden. Das teilweise Ausweichen auf jüngere Bäume führte zu dem - im Vergleich zu den beprobten Eschen und Hainbuchen - geringeren Durchschnittsalter der untersuchten Buchen von 105 Jahren (vgl. Abb. 4.21, Kap. 4.4.2). Dieses Alter entspricht dem mittleren Alter der Winterlinden (104 
Jahre). Für diese Art repräsentiert der Wert allerdings das tatsächliche Durchschnittsalter der Kronenbäume, das für alle Flächen zwischen 100 und 120 Jahren liegen dürfte. Die Winterlinden weisen die geringste Altersspanne auf (75-127 Jahre). Das relativ einheitliche Alter der beprobten Individuen könnte ein Grund für den Kurvenverlauf der Zuwachsentwicklung dieser Art sein, der zu Beginn (1900 - 1920 s. Abb. 4.22, Kap. 4.4.2) den typischen Verlauf des juvenilen Wachstums zeigt.

Der ab den 1970er Jahren deutliche Zuwachsrückgang der Linden, der sich auch bei den Hainbuchen zeigt, kann allerdings nicht auf einen Alterstrend zurückgeführt werden, da beide Arten sich mit 70 bis 110 Jahren noch in der Optimalphase ihrer Entwicklung befinden. Zudem überlagern Klima- und Konkurrenzeffekte den Alterstrend bei der Jahrringbildung adulter Bäume stark (LE GOFF \& OTTORINI 1993, BOURIAUD 2004). Ein möglicher Grund für den Zuwachsrückgang könnte bestandesgeschichtlicher Natur sein. Wie in Kapitel 5.1 bereits erwähnt, wurde der Bereich 1961 zum Naturschutzgebiet erklärt und 1964 zum militärischen Sperrgebiet. Der damit einhergehende Nutzungsrückgang führte zu einer sich verstärkenden Konkurrenz im Kronenraum, auf die diese beiden Baumarten als erste reagierten. Die Esche zeigt seit dieser Zeit einen starken Anstieg der Ringbreiten - mit Ausnahme der Jahre 1970-75 - und reagierte somit positiv auf die bestandesgeschichtliche Entwicklung. Sie ist die einzige Art, die keinen signifikanten Zuwachsrückgang in den letzten 40 Jahren verzeichnete.

Der drastische Rückgang des Stammwachstums der Buche fällt mit dem Trockenjahr 1983 zusammen. Die Reaktion der Buche in den Folgejahren sind eingehend untersucht worden. Der Zuwachseinbruch erfolgte oft erst im Folgejahr und konnte je nach Vitalität des Bestandes und der Standortsbedingungen erst nach einigen Jahren wieder kompensiert werden (DitTMAR \& Zech 1994, DitTMAR 2003, Lebourgeois et al. 2005). Die starke Reaktion der Buche muss in Verbindung mit der Häufung von Trocken-Perioden ab 1976 gesehen werden, die vor allem Buchen auf klimatischen und pedologischen Grenzstandorten bereits schwächten (ASTHALTER 1984, ECKSTEIN 1984, BRAUN \& FLÜCKINGER 1987, FisCHER \& ROMMEL 1989). Entgegen der Vermutung, dass zunehmende Immissionsbelastungen mitverantwortlich für die Zuwachsrückgänge seien, belegen zahlreiche Untersuchungen, dass klimatische Einflüsse - kombiniert mit Konkurrenzeffekten - die Hauptursachen für die Reaktion der Buche und der ebenfalls intensiv untersuchten Fichte sind (WorBES 1995, LE GofF \& OTTORINi 1993, CESCATTI \& PiUtTI 1998).

Dabei spielt in Hochlagen vor allem die Temperatur eine wichtige Rolle (DITTMAR \& ZECH 1994), in Tieflagen ist demgegenüber vor allem der Niederschlag während der 
Vegetationsperiode und im Herbst des Vorjahres entscheidend (ECKSTEIN 1984, LE GOFF \& OTTORINI 1993, LEBOURGEOIS 2005). Für die im Hainich untersuchten Buchen hatte ebenfalls die Niederschlagsumme von März bis August des aktuellen Jahres den größten Einfluss auf die Ringbildung, jedoch konnte das Ergebnis durch Einbeziehung der Mitteltemperatur für diesen Zeitraum noch verbessert werden (s. Abb. 4.24, Kap. 4.4.2). Zu dem gleichen Ergebnis kommt auch BONN (1998), der für Buchen auf mittleren und trockenen Standorten in Mittelund Norddeutschland mit dem gleichen Klimaindex Korrelationen von $\mathrm{r}=0.29$ - 0.65 berechnete. Das Ergebnis verbesserte sich nach BonN (1998) um durchschnittlich 5\%, wurde der Klimaeinfluss des Vorjahres mit berücksichtigt. Dies war für die hier untersuchten Buchen nicht der Fall. Jedoch liegen die Endergebnisse von BonN (1998) mit r=0.53 (mit Einfluss des Vorjahres) sehr dicht an dem für die Buchen im Hainich berechneten Wert von $\mathrm{r}=0.52$.

Tab. 5.6: Klimakorrelationen, Autokorrelation (AC) und mittlere Sensitivität (MS) der Buche auf unterschiedlichen Standorten in Mitteleuropa nach verschiedenen Autoren.

\begin{tabular}{|c|c|c|c|c|c|c|}
\hline Autoren & $\begin{array}{l}\text { Untersuch- } \\
\text { ungskriterien }\end{array}$ & Klimafaktor & $\begin{array}{l}\text { Korrela- } \\
\text { tions- } \\
\text { koeffizient } \\
\end{array}$ & $\mathrm{AC}$ & MS & $\begin{array}{l}\text { Zeit- } \\
\text { intervall }\end{array}$ \\
\hline $\begin{array}{l}\text { Eckstein et } \\
\text { al. } 1984\end{array}$ & Schadstufen & $\begin{array}{l}\text { Niederschlag. } \\
\text { und Temp. } \\
\text { ganzes Jahr und } \\
\text { Vorjahr }\end{array}$ & $\begin{array}{l}\text { Keine } \\
\text { Angabe }\end{array}$ & $\begin{array}{l}0.27-0.75 \\
\text { MW: } 0.51\end{array}$ & $\begin{array}{l}0.14-0.34 \\
0.21\end{array}$ & $1922-1980$ \\
\hline Bonn 1998 & $\begin{array}{l}\text { Trockenheits- } \\
\text { gradient \& } \\
\text { Artenvergleich }\end{array}$ & $\begin{array}{l}\text { Niederschlag } \\
\text { und Temp. } \\
\text { (Veg-Per.) } \\
\text { z.T. mit Vorjahr }\end{array}$ & $\begin{array}{l}0.41-0.71 \\
\text { MW: } 0.53\end{array}$ & 0.63 & $\begin{array}{l}0.19-0.38 \\
\text { MW: } 0.26\end{array}$ & 1934-1993 \\
\hline $\begin{array}{l}\text { Dittmar \& } \\
\text { Zech } 1994\end{array}$ & $\begin{array}{l}\text { Schadstufen } \\
\text { Hier zit. nur } \\
\text { Tieflagen }\end{array}$ & $\begin{array}{l}\text { Niederschlag } \\
\text { (Feb.-Juni) }\end{array}$ & $\begin{array}{l}0.11 \text { (n.s.)- } \\
0.44\end{array}$ & $0.68-0.83$ & $0.15-0.19$ & 1902-1992 \\
\hline $\begin{array}{l}\text { Dittmar et } \\
\text { al. } 2003\end{array}$ & $\begin{array}{l}\text { Höhengradient } \\
\text { Hier zit. nur } \\
\text { Tieflagen in } \\
\text { Mittel-europa }\end{array}$ & $\begin{array}{l}\text { Niederschlag } \\
\text { und Temp. } \\
\text { z.T. mit Vorjahr }\end{array}$ & $\begin{array}{l}0.52-0.83 \\
\text { MW: } 0.66\end{array}$ & $\begin{array}{l}0.52-0.79 \\
\text { MW:0.67 }\end{array}$ & $\begin{array}{l}0.20-0.39 \\
\text { MW: } 0.27\end{array}$ & 1952-1995 \\
\hline $\begin{array}{c}\text { Lebourge- } \\
\text { ois } 2005\end{array}$ & Höhengradient & $\begin{array}{l}\text { Bodenwasser- } \\
\text { gehalt (Veg.- } \\
\text { Per.) }\end{array}$ & $0.47-0.59$ & $0.40-0.67$ & $0.19-0.32$ & 1949-1994 \\
\hline Frech 2006 & Artenvergleich & $\begin{array}{l}\text { Niederschlag } \\
\text { und Temp. } \\
\text { (Veg-Per.) }\end{array}$ & 0.52 & 0.70 & 0.20 & $1951-2004$ \\
\hline
\end{tabular}

Auch andere Autoren kommen unter Einbeziehung des Vorjahres (ECKSTEIN 1984, DITTMAR et al. 2003) oder auch nur des Jahres der Ringbildung (DITTMAR \& ZECH 1994, LEBOURGEOIS 2005) zu ähnlichen Ergebnissen (s. Tab. 5.6) mit Korrelationskoeffizienten um 0.44 bis 0.66 für Buchen in Mitteleuropa auf unterschiedlichen, jedoch nicht extremen Standorten. Nach 
LeBourgeois (2005) hat der Bodenwassergehalt im Juni den größten Einfluss auf das Dickenwachstum. Dieser Faktor allein erklärt 26.6\% der Gesamtvarianz der Ringbreiten. Detaillierte Zuwachsuntersuchungen bestätigen, dass die Hauptwachstumsperiode der Buche im Juni und in der ersten Julihälfte liegt (SCHIPKA 2002), diese kann aber unter günstigen Bedingungen bis in den September hinein verlängert werden (KRAMER 1980).

Alle Autoren weisen nach, dass die Buche mit Werten der Mittleren Sensitivität (MS) zwischen 0.15 und 0.38 sehr klimasensitiv ist. Die Sensitivität ist ein in der Dendrochronologie häufig verwendetes Maß (FRITTS 1976, SCHWEINGRUBER 1996) für die Empfindlichkeit eines Baumes oder der Bäume eines Standortes gegenüber Umwelteinflüssen und ermöglicht den direkten Vergleich verschiedener Standorte unabhängig von den Größenordnungen der absoluten Werte der Zeitreihen. Auf den gleichen Standorten zeigten Eichen (Quercus spec. L.) in der Untersuchung von BonN (1998) eine mit 0.18 deutlich geringere Sensitivität als Buchen (0.26) und zugleich eine höhere Autokorrelation (0.78 gegenüber 0.64 für Buche). Die Autokorrelation einer Zeitreihe ist die Korrelation der Reihe mit sich selbst in phasenverschobener Lage. Die hier verwendete Autokorrelation mit einjähriger Phasenverschiebung zeigt den Zusammenhang zwischen dem Zuwachs des laufenden Jahres und dem Vorjahr. Der Informationsgehalt der Autokorrelation ist mit dem der Sensitivität gekoppelt. Bei einer erhöhten Empfindlichkeit gegenüber Umwelteinflüssen und damit erhöhter Sensitivität werden der Vorjahreseinfluss und damit die endogenen Wuchseigenschaften überlagert.

Von den vier untersuchten Baumarten weist die Buche die größte Sensitivität auf (s. Tab. 4.8, Kap. 4.42). Diese nimmt in der Reihenfolge Esche (0.17) > Hainbuche (0.16) > Winterlinde (0.12) ab. In dieser Reihenfolge nimmt auch der Zusammenhang zwischen Klima und Ringbreite ab (vgl. Abb.4.24, Kap. 4.42). Dieser ist für die Winterlinde nicht signifikant. Zusammen mit der äußerst geringen Sensitivität und der hohen Autokorrelation zeigt sich diese Art als weitgehend unabhängig von klimatischen Schwankungen. Das macht sie gegenüber Extremereignissen sehr resistent. So ist beispielsweise der Zuwachsrückgang im Trockenjahr $1976 \mathrm{im}$ Vergleich zu den anderen Arten sehr gering (s. Abb. 4.23, Kap. 4.42).

Leider fehlen vergleichbare Studien, da die Winterlinde bislang kaum dendroökologisch untersucht wurde. Aufgrund des weichen Holzes, das sich bei der Probenahme leicht verzieht und den sehr schwach ausgeprägten Jahrringgrenzen bei zerstreutporiger Gefäßanordnung (SACHSSE \& SCHWAER 1991) eignet sich die Art nicht für dendrologische Studien und wird von HÖFLER \& ELLING (1966) wegen der häufigen Ausfälle von Jahrringen als nicht auswertbar eingestuft. Dies gilt auch für Verwendung der Art in Hinblick auf archäologische 
Fragestellungen (GUYAN 1977). So beschränken sich Untersuchungen, in denen die Winterlinde miteinbezogen ist, auf holzanatomische Untersuchungen (ISELI \& SCHWEINGRUber 1989, SACHSSE \& Schwaer 1991, Grosser \& GRAeSSLE 1976) und Waldbrandstudien (BERLI \& SCHWEINGRUBER 1992). Einzeluntersuchungen nutzen das hohe Alter solitärer Linden für langfristige ${ }^{14}$ C-Analysen (ATTOLINI et al. 1989).

Auch die Hainbuche ist für dendrologische Untersuchungen wenig geeignet. Die Jahrringgrenzen sind ebenfalls nur schwach erkennbar und zudem oft gewellt, was die Analyse sehr erschwert. In der dendrologischen Arten-Datenbank (erstellt von GRISSINOMAYER \& DOBBERTIN 2003), in der alle bisher untersuchten Arten aufgeführt und hinsichtlich ihrer Eignung zum cross-dating klassifiziert sind (CDI-Index $0-2$ ), wird die Hainbuche der Klasse 0 zugeordnet (http://www01.wsl.ch/species/index.html) und damit als äußerst ungeeignet eingestuft. Dementsprechend liegen auch für die Hainbuche keine Vergleichsstudien vor. Lediglich WEITLAND (1960) untersuchte in einer vergleichenden Studie neben Rotbuchen und Stieleichen auch einige Hainbuchen und Eschen in Norddeutschland. Aus der Studie geht hervor, dass die Hainbuche mit 0.22 eine höhere Sensitivität als die Rotbuche (0.20) und als die Esche (0.17) aufweist. Der Datensatz beschränkt sich jedoch auf jeweils vier Individuen. Einzelne Individuen untersuchte auch CIESLAR (1907) und zeigt deutliche Zuwachseinbrüche der Hainbuche, aber auch der Rotbuche im Trockenjahr 1904, wohingegen die untersuchten Eschen - auf feuchten Standorten - weitgehend unbeeinträchtigt bleiben.

Aus den hier vorgestellten Untersuchungen lässt sich ableiten, dass auch die Hainbuche weniger klimasensitiv und auch trockenheitsresistenter ist als die Buche. Bei geringer mittlerer Sensitivität (0.16) weist sie jedoch - im Gegensatz zur Winterlinde - auch eine geringere Autokorrelation auf. Damit ist sowohl der Einfluss des Klimas als auch der Einfluss der Vorjahresbedingungen relativ gering, was darauf hindeutet, dass ein bislang nicht berücksichtigter Faktor das Wuchsverhalten mitbestimmt. Die Hainbuche weist die geringsten durchschnittlichen Ringweiten auf und auch das geringste BHD-Altersverhältnis, was darauf hindeutet, dass diese Art besonders durch Konkurrenz in ihrem Wachstum beeinträchtigt wird. Der Einfluss der Konkurrenz ist in dendroökologischen Untersuchungen nur schwer von anderen Einflussfaktoren $\mathrm{zu}$ trennen und $\mathrm{zu}$ quantifizieren. Es gibt allerdings einige vielversprechende Ansätze, statistische Nachweismethoden zu entwickeln (z.B. MELDAHL et al. 1999, PiutTi \& CesCATti 1999). 
Die Esche eignet sich - aufgrund ihrer deutlichen Jahrringgrenzen und der ringporigen Struktur - wiederum sehr viel besser für Jahrringanalysen. Jedoch waren auch für diese Art nur wenige vergleichbare Untersuchungen $\mathrm{zu}$ finden. CALTON \& FLETCHER (1978) untersuchten die Zuwächse von Stieleichen und Eschen im gleichen Bestand. Beide Arten zeigten sich in ihrem Wuchsverhalten unabhängig voneinander. Die untersuchten Eschen zeigten eine deutlich größere Abhängigkeit vom Niederschlag in den Monaten Mai bis Juli als die Eiche. Ein verstärkter Einfluss der Temperatur war, wie für die in dieser Arbeit untersuchten Eschen im Hainich, nicht feststellbar.

Die untersuchten Eschen im Hainich weisen in den letzten zehn Jahren im Gegensatz zu Buchen, Hainbuchen und Winterlinden keinen negativen Wachstumstrend auf und erzielten die größten Zuwächse. Somit scheint die Esche von den derzeitigen Standortsbedingungen am stärksten zu profitieren. 


\subsection{Autökologische Eigenschaften der Rotbuche, Esche, Hainbuche und Winterlinde und ihre mögliche Rolle in der künftigen Waldentwicklung}

Die Reaktion der Buche auf extreme Trockenperioden und ihre klimatische Belastbarkeit wurde an vielen Standorten untersucht (z.B. ECKSTEIN et al. 1984, FELBERMEIER 1994, DuQuesnay et al. 1998, Bonn 1998, DitTMAr et al. 2003, RENNEnBerg et al. 2004, LEBOURGEOIS et al. 2005). Viele Autoren weisen auf die sich verändernden Zuwächse in den letzten Jahrzehnten hin. Diese Veränderungen werden in direkter Verbindung mit dem globalen Klimawandel gesehen, der sich unterschiedlich auf die verschiedenen Regionen auswirkt. Dabei spielen eine erhöhte $\mathrm{CO}_{2}$-Konzentration auf der einen Seite und erhöhte Temperaturen sowie veränderte Niederschlagsregime auf der anderen Seite eine wichtige Rolle (IPCC 2001). Nicht zuletzt die unterschiedlichen Auswirkungen dieser Parameter macht eine Vorhersage oder Modellierung dieser Einzelaspekte des Klimawandels äußerst schwer (SCHENK 1996, MEDLYN et al. 1999). Hinzu kommt, dass es nicht nur zu einer langfristigen Änderung der mittleren Klimabedingungen und auch $\mathrm{zu}$ einer Verlängerung der Vegetationsperiode kommen wird, sondern auch eine Häufung extremer Witterungsereignisse, wie Trockenperioden und Stürme, zu erwarten ist (FABIAN \& MENZEL 1998). Langfristige Veränderungen können zu einer Verschiebung der Arealgrenzen einzelner Arten führen (KIRSCHBAUM et al. 1996, LEUSCHNER \& SCHIPKA 2004), die Häufung starker Störereignisse kann jedoch zu einem sehr viel abrupteren Artenwechsel innerhalb eines Ökosystems führen.

Die Auswirkungen beider Aspekte auf Buchenwälder Mitteleuropas sind von vielen Seiten untersucht worden. So weisen CESCATTI \& PiUTTI (1998) nach, dass frei gestellte Buchen deutlich weniger sensibel auf Trockenperioden reagieren und somit waldbauliche Maßnahmen zur Stabilisierung der Wälder beitragen können. PETERS \& POULSEN (1994) sowie WoHLgemuth et al. (2002) zeigen, wie sich die Störungsintensität auf die Artenzusammensetzung von Buchenwäldern auswirkt. Beide Autoren stimmen darin überein, dass eine hohe Störungsintensität zu einer Dominanzreduktion der Buche führt und der Anteil anderer Baumarten zunehmen wird. Eine daraus ableitbare waldbauliche Maßnahme ist das aktive Einbringen z.B. trockenheitsresistenterer Arten in Buchenreinbestände, um eine nachhaltige Stabilisierung der Ökosystemfunktionen des Waldes zu gewährleisten (z.B. RÖHRIG 1966, Asthalter 1984). Doch obwohl die Begründung von Mischwäldern längst erklärtes Ziel der naturnahen Waldwirtschaft geworden ist, gibt es erstaunlich wenig 
Untersuchungen zur Autökologie alternativer Laubholzarten, die z.B. eine höhere Trockenheitsresistenz als die Buche besitzen (HöLSCHER et al. 2005). Die im Hainich untersuchten Arten Esche, Winterlinde und Hainbuche zeigen sich weniger klimasensitiv als die Buche und kommen somit als mögliche Neben- bzw. Ersatzbaumarten in Frage. Aus diesem Grund sollen hier zusammenfassend einige der wichtigsten autökologischen Eigenschaften dieser Arten auf der vorhandenen Datengrundlage denen der Buche gegenübergestellt werden.

\subsubsection{Fagus sylvatica}

Die Buche befindet sich an der Ostabdachung des Hainich am östlichen Rand ihrer Verbreitungsgrenze, die durch das angrenzende Thüringer Becken in diesem Bereich sehr weit nach Westen verlagert ist. Die Niederschläge liegen im Mittel der letzten 32 Jahre (19732004) bei $591 \mathrm{~mm}$ im Jahr. Dabei lagen die Jahressummen der Niederschläge in elf Jahren unter 500 mm. Nach Ellenberg (1996) liegt die Trockenheitsgrenze der Buche bei $600 \mathrm{~mm}$. Die Trockenheitstoleranz ist jedoch auch abhängig von der Temperatur und dem damit in Verbindung stehenden Wasserdampfsättigungsdefizit der Luft. Mit einem Index aus Jahresniederschlag und tausendfacher Mitteltemperatur des wärmsten Monats kann nach EllenBerg (1996) die Toleranzgrenze der Buche gegenüber Trockenheit berechnet werden. Diese ist nach Ellenberg dort erreicht, wo langfristig Werte über 30 auftreten.

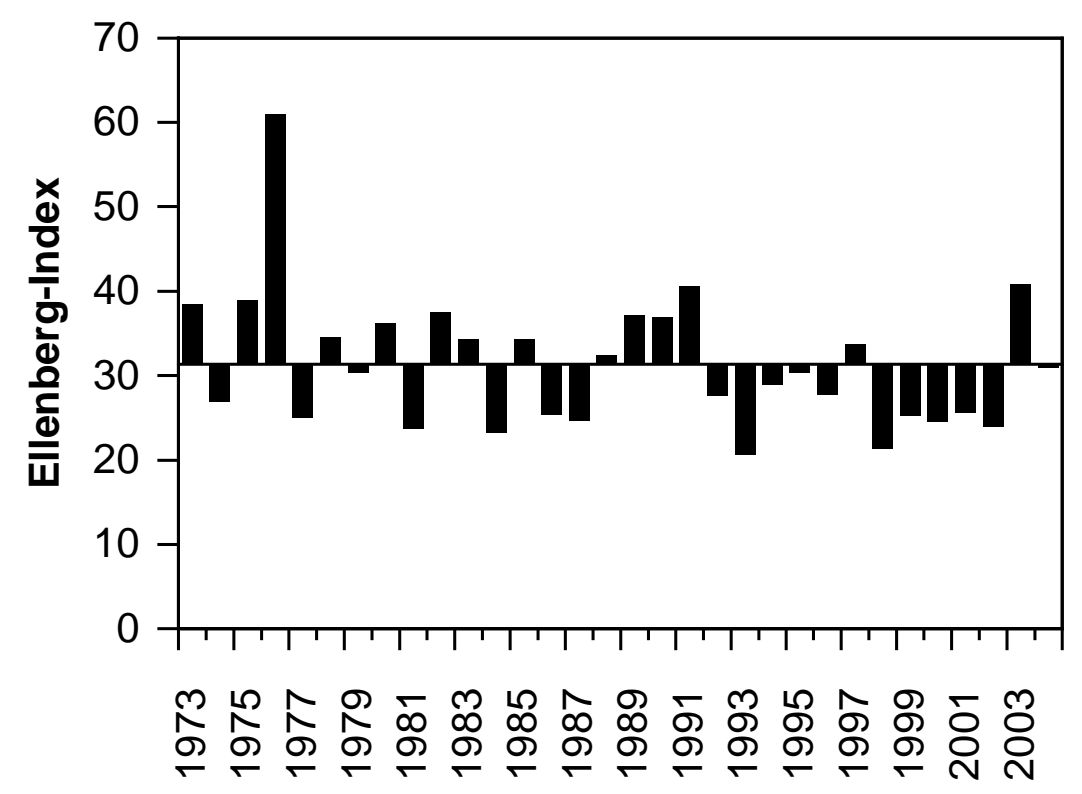

Abb. 5.3: Nach ELLENBERG (1996) berechneter Quotient aus tausendfacher Mitteltemperatur des wärmsten Monats und jährlicher Niederschlagssumme aufgetragen als Abweichung vom Mittelwert (31.35) zur Indexierung der Toleranzgrenze der Rotbuche, die ELLENBERG (1996) bei Werten von über 30 ansetzt. 
Der für die Flächen im Hainich berechnete Mittelwert liegt bei 31.35, also leicht über der potentiellen Toleranzgrenze der Buche. In den vergangen 32 Jahren lag der Wert zu 50\% unter und zu 50\% über 30 (s. Abb. 5.3).

Das Jahr 2004 spiegelt mit einem Wert von 30.9 fast genau die mittleren Standortverhältnisse wieder. Im Jahresvergleich tritt das extreme Trockenjahr 1976 mit einem Index-Wert von 61 besonders deutlich hervor. Auch die gravierende Trockenheit des Jahres 1959 reicht daran nicht heran. In diesem Jahr wurden viele Buchen im Bestand, als Konsequenz der starken Schwächung, von Schleimfluss befallen (pers. Mitteilung, ehem. Revierförster FUCHS).

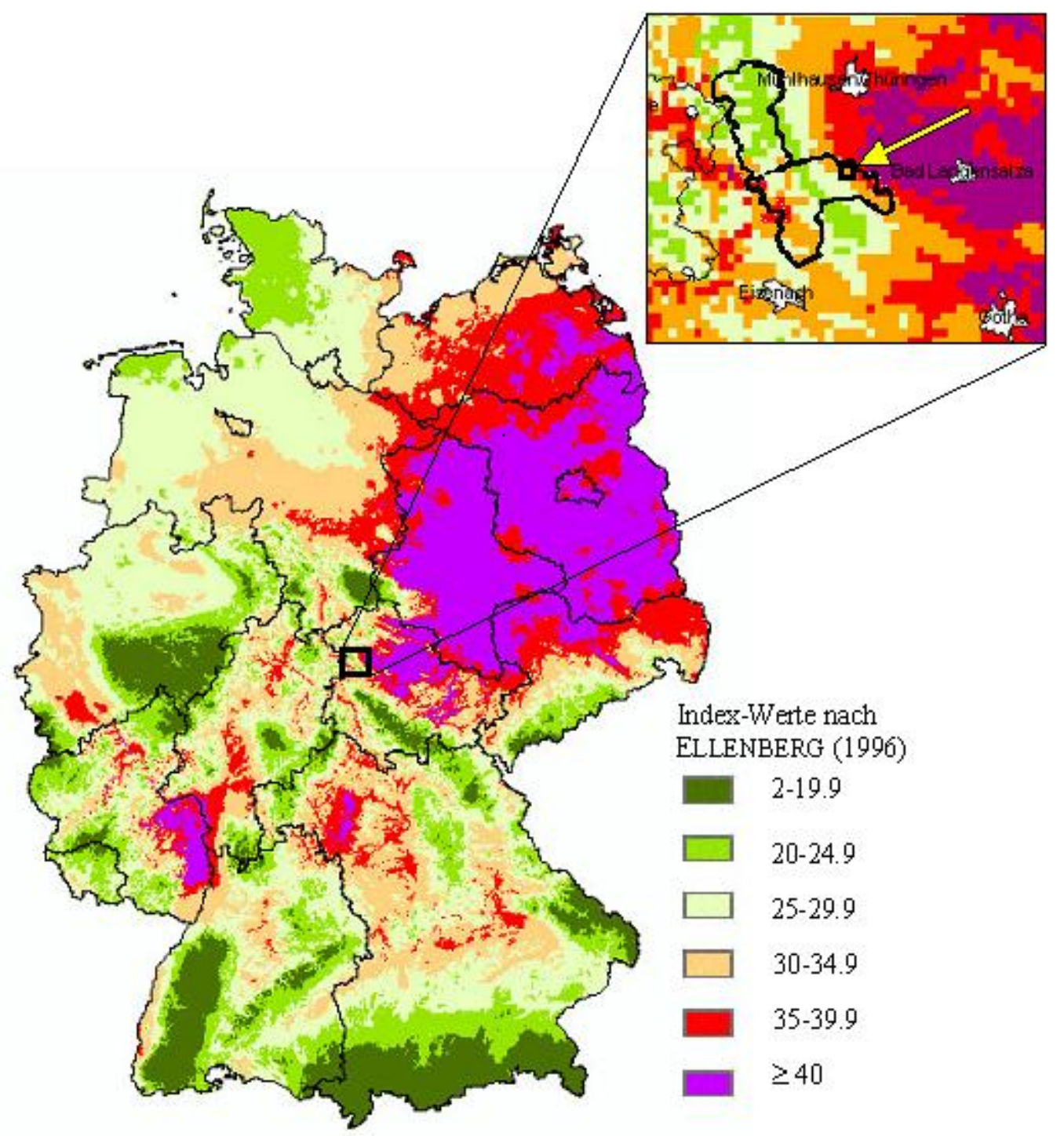

Abb. 5.4: Bundesweite Verteilung des nach ELLENBERG (1996) berechneten Quotienten aus tausendfacher Mitteltemperatur des wärmsten Monats und jährlicher Niederschlagssumme gemittelt über die Jahre 1961-1990. Hervorgehoben ist der Höhenzug des Hainich (vgl. Abb. 2.1). Der gelbe Pfeil weist auf das Untersuchungsgebiet. Die Grafik wurde erstellt und zur Verfügung gestellt von Prof. Bareth, Universität Köln auf Grundlage der Daten des DEUTSCHEN WeTtERDIENSTES (1999, 2000, 2003). 
Der in Abbildung 5.4 gegebene Überblick über die deutschlandweite Verteilung der IndexWerte zeigt die grundsätzlich anderen Klimabedingungen im Nordosten Deutschlands. Der Ostrand des Hainich zeigt einen sehr steilen Gradienten der Klimaänderung vom Höhenzug hin zum Thüringer Becken und zeigt damit sehr deutlich die unterschiedlichen Standortsbedingungen der Buche im Nationalparkgebiet.

Der dargestellte Index-Wert ist jedoch nur als grobe Annäherung an die Toleranzgrenze der Buche hinsichtlich Temperatur und Niederschlag zu sehen. Es gilt mittlerweile als fragwürdig, ob die von Ellenberg festgesetzte Toleranzgrenze der Buche von $600 \mathrm{~mm}$ Jahresniederschlag aufrecht zu erhalten ist. Untersuchungen an Buchen im Mitteldeutschen Trockengebiet bei Niederschlagssummen um $500 \mathrm{~mm}$ zeigen keine deutlichen strukturellen oder morphologischen Anpassungsmechanismen der Buche zum Beispiel durch eine Abnahme der Bestandestranspiration, der Blattfläche oder einer Zunahme der Feinwurzelbiomasse (HERTEL et al. 2004). Die Autoren stellen daher die Hypothese auf, dass die heutige Verbreitungsgrenze der Buche in Ost- und Mitteldeutschland nicht der potentiell natürlichen Trockenheitsgrenze dieser Art entspricht, sondern durch andere - auch anthropogene - Einflüsse zustande gekommen ist.

SCHIPKA et al. (2005) weisen jedoch für den gleichen Bestand im mitteldeutschen Trockengebiet eine Limitation der Bestandestranspiration durch den Bodenwassergehalt und deutlich geringere Zuwächse der Art als in niederschlagsreicheren Gebieten Mittel- und Nordwestdeutschlands nach.

Untersuchungen von LEUSCHNER et al. (2001) weisen ebenfalls starke Reaktionen der Buche in einer Trockenperiode im Sommer 1995 nach (deutlicher Zuwachsrückgang und erhöhte Feinwurzelmortalität), die an benachbarten Traubeneichen nicht beobachtet werden. Die Autoren schließen daraus jedoch nicht auf eine generelle Konkurrenzunterlegenheit der Buche auf diesem Standort in der Lüneburger Heide, der aufgrund seiner edaphischen Verhältnisse ebenfalls als Grenzstandort für die Buche galt. Auch BoNN (1998) kommt in seiner vergleichenden dendroökologischen Studie zwischen Buchen und Eichen im gleichen Gebiet zu dem Schluss, dass die weitaus höhere Klimasensitivität der Buche bislang nicht zu einer Schwächung dieser Art geführt hat, sondern sie im Gegenteil in der interspezifischen Konkurrenz der Eiche weitaus überlegen ist.

Daraus lässt sich folgern, dass die Standortamplitude der Buche größer ist als bisher angenommen. Vor dem Hintergrund des prognostizierten Klimawandels wird dementsprechend wohl weniger der Anstieg der mittleren Temperaturen ausschlaggebend sein (FELBERMEIER 1994), sondern vielmehr die Häufigkeit und Intensität von Extremereignissen 
und dabei vor allem von Trockenperioden über die zukünftige Konkurrenzkraft der Buche entscheiden.

\subsubsection{Fraxinus excelsior}

Die physiologische Amplitude der Esche in Bezug auf den Wasserhaushalt ist weiter als die der Buche. Eschen können sowohl feuchtere - auch staunasse - als auch trockenere Standorte besiedeln als die Buche (RöHrig 1992, Claessens et al. 1999). Aufgrund der sehr guten Ausbreitungsmechanismen ist die Esche in der Lage, brach gefallene landwirtschaftliche Flächen und Flussufer schnell zu besiedeln, was zu einer sehr starken Ausbreitung der Art in den letzten Jahrzehnten geführt hat (JOHNSON 1988, MARIGO et al. 2000). Allerdings benötigt sie basenreiche Böden und ist in dieser Hinsicht sehr viel anspruchsvoller als die Buche. Die Bedingungen im Hainich sind für diese Art somit nahezu optimal. In der langen Trockenperiode im Sommer 2003 zeigte die Esche als einzige Art keinerlei Anzeichen von Trockenstress, während Buchen mit Verfärbungen der Blätter und frühzeitiger Blattseneszenz reagierten und einige Kronen schon Ende August fast vollständig entlaubt waren. Die besondere Resistenz dieser Art gegenüber periodischer Bodenaustrocknung belegen auch Saftfluss-Messungen, die auf einer ebenfalls im Hainich gelegenen Fläche durchgeführt wurden (HÖLSCHER et al. 2005). Während einer sommerlichen Trockenperiode im Jahr 2001 zeigte die Esche als einzige Art keine signifikante Absenkung der Saftflussdichte bei abnehmendem Bodenwassergehalt und steigendem Wasserdampfsättigungsdefizit der Luft (VPD), während die Buche die Saftflussrate um 44\% herabsenkte im Vergleich zu Tagen mit gleichem VPD aber hohem Bodenwassergehalt. Allerdings wies die Esche mit $67 \mathrm{~g} \mathrm{~cm}^{-2}$ pro

Tag insgesamt sehr viel geringere mittlere Saftflussraten auf als die Buche $\left(152 \mathrm{~g} \mathrm{~cm}^{-2}\right.$ pro Tag) und eine 11-fach kleinere wasserleitende Xylemfläche.

Die Anpassungsmechanismen an Trockenheit sind bei der Esche vor allem in der Regulation der stomatären Leitfähigkeit und einem starken Absenken des osmotischen Potentials zu sehen. Bei beiden Prozessen spielt Malat eine Schlüsselrolle (MARIGO et al. 2000).

Eine weitere wichtige Rolle in der Trockenheitstoleranz spielt das relativ große Wurzelsystem der Esche. Dieses ist extrem flachgründig ausgeprägt mit einigen vertikalen Senkwurzeln. Bei Messungen des Wurzelsaftflusses zeigte die Esche eine um 66\% höhere oberflächenspezifische Saftflussrate als die Buche im gleichen Bestand im Hainich (KoRN 2004). Die hohe Feinwurzeldichte dieser Art, die schon bei Jungpflanzen bei Eschen deutlich höher ist als bei Buchen, könnte ebenfalls ein wichtiger Faktor zur Steigerung der 
Trockenheitsresistenz sein (RUST \& SAVILL 2000, STÖHR \& LÖSCH 2004, HÖlSCHER et al. 2005).

Die Esche gilt in ihrer Jugend als sehr schattentolerant. So haben Eschensämlinge im Bestandesschatten eine sehr hohe Überlebensdauer, langfristig im Bestand etablieren können sich Jungbäume aber nur unter guten Lichtbedingungen (TAPPER 1996). HÖLSCHER (2003) weist einen grundsätzlichen Wandel der Photosyntheseleistung zwischen Eschen-Sämlingen und Altbäumen nach.

Die im Rahmen dieser Arbeit durchgeführten Verjüngungsaufnahmen zeigen, dass die Esche sich überproportional zur Abundanz der Altbäume auf allen Flächen gut verjüngt und keine Abhängigkeit der Sämlingsdichte von der Relativen Beleuchtungsstärke am Waldboden besteht. Jungbäume über $50 \mathrm{~cm}$ sind demgegenüber selten. Die schon von HuldÉN (1941) beschriebene und von OTTORINI et al. (1996) bestätigte Strategie der Esche, unter günstigen Lichtbedingungen sehr schnell in die Höhe zu wachsen und erst nach Erreichen der Bestandeshöhe die Krone auszubreiten, konnte im Hainich ebenfalls beobachtet werden (vgl. Abb. 4.9, Kap. 4.2.1). In diesem Stadium des Höhenwachstums investieren die Jungbäume auch nicht in eine Ausweitung des Grobwurzelsystems, was sie extrem windanfällig macht. Schon aus diesem Grund eignet sich die Art nicht zur Reinbestandsbegründung (RöHRIG 1966), obwohl die Esche im Reinbestand die besten Zuwächse erzielt (MAURER 1965). In der Krone ist die Esche aufgrund ihrer dicken Zweige und dem sehr weiten Schwankungsradius, den die Einzelkronen im Wind erreichen, eine Art, die Bäume mit feineren Endtrieben in direkter Nachbarschaft stark schädigen kann (vgl. Kap. 4.3.3). Aufgrund ihrer Eigenschaft, meist über die Kronen der Nachbarbäume hinauszuwachsen und gleichzeitig durch die weniger dichte Belaubung (LE GOFF \& OTTORINI 1996) noch relativ viel Licht in tiefere Bestandesschichten dringen zu lassen, erweist sich die Esche langfristig jedoch als sehr gute Mischbaumart. In Mischung mit anderen schattentoleranten Arten mit anderer Kronenarchitektur besteht die Möglichkeit zur Bildung eines Bestandes mit sehr dicker Kronenschicht durch einen geschichteten Bestandesaufbau (s. Abb. 5.2, Kap. 5.2.2). Die dendroökologischen Untersuchungen zeigen zudem, dass die Esche von allen Baumarten unter dem durch die Einstellung der Bewirtschaftung bewirkten Anstieg der Konkurrenzintensität am wenigsten beeinträchtigt ist und in den letzten 10 Jahren die höchsten Zuwächse erzielte (s. Abb. 4.22, Kap. 4.4.2).

Experimentelle Untersuchungen an Eschen unter erhöhten $\mathrm{CO}_{2}$ - und Ozon-Konzentrationen in der Luft ergaben zudem, dass die durch das erhöhte $\mathrm{CO}_{2}$-Angebot bedingte Wachstumssteigerung von Eschensämlingen von 20\% sich, im Gegensatz zu Kiefern und 
Traubeneichen, durch gesteigerte Ozongehalte nicht wieder reduzierte (BROADMEADOW \& JACKSON 2000). Die Auswirkungen auf das Wurzelwachstum unter erhöhtem $\mathrm{CO}_{2}$-Angebot $\left(700 \mu \mathrm{mol} \mathrm{mol}{ }^{-1}\right)$ waren im Vergleich dieser drei Arten für die Esche am geringsten. Die totale Wurzelbiomasse steigerte sich bei der Esche am wenigsten, obwohl auch diese Art erhöhte Fein- und Grobwurzelbiomassen aufwies (CROOKSHANKS et al. 1998). Vergleichende Untersuchungen von Buchen und Eschen fehlen bislang. Vor diesem Hintergrund lässt sich nur vermuten, dass die Esche das Potenzial hat, die durch die prognostizierte Temperaturerhöhung von bis $\mathrm{zu} \quad 3^{\circ} \mathrm{C}$ bedingte Ausweitung ihres potenziellen Verbreitungsgebietes in Nordeuropa und der montanen Stufe zu realisieren und diesen Raum tatsächlich zu besiedeln.

\subsubsection{Carpinus betulus}

Das Verbreitungsgebiet dieser Art umfasst West-, Mittel- und Südeuropa und deckt sich in großen Teilen mit demjenigen der Rotbuche. Es geht weniger weit nach Norden, jedoch sehr viel weiter nach Nordosten und Osten (LEIBUNDGUT 1991). Ob das häufige Vorkommen dieser Art in kontinental getönten Klimabereichen auf eine bessere autökologische Adaptation an die dortigen Bedingungen zurückzuführen ist, ist hingegen fraglich. Vielmehr wird angenommen, dass das heutige Vorkommen der Art stark von der Ausbreitungssituation während der Nacheiszeit geprägt ist (KÜSTER 1996), da die Hainbuche in der letzten Warmzeit, im Eem, keineswegs im Osten stärker vertreten war als im Westen, sondern, ganz im Gegenteil, ihren Verbreitungsschwerpunkt auf den Britischen Inseln und in Nordwestdeutschland hatte (LANG 1994). Im Holozän dagegen kam die Rotbuche der Hainbuche bei der Wiederbesiedlung des westlichen Mitteleuropas zuvor, so dass die Hainbuche auf weiter östlich gelegene Areale auswich, die von der Rotbuche nicht besiedelt wurden (KÜSTER 1997). Die heutigen Vorkommen der Hainbuche im westlichen Mitteleuropa sind zu einem großen Teil durch die im frühen Mittelalter einsetzende Nieder- und Mittelwaldwirtschaft bedingt, da die Hainbuche wegen ihrer Ausschlagfreudigkeit bevorzugt als dienende Baumart unter Eiche als Unterwuchs eingebracht wurde (RUBNER 1960). Die daraus hervorgegangenen heutigen EichenHainbuchen-Wälder sind also in vielen Fällen als Ersatz von ursprünglichen Buchen- und Buchen-Eichenwäldern aufzufassen (KÜSTER 1996).

Guten Wuchs zeigt die Hainbuche nur auf frischen, mineralreichen Böden mit genügendem Tongehalt (BARTELS 1993). Im Bestand zeichnet sich die Art durch eine große Schattentoleranz bis ins hohe Alter aus (ROLOFF 1988). Allerdings benötigen die Keimlinge für eine erfolgreiche Verjüngung mehr Licht als Buchen und Linden in diesem Alter (RÖHRIG \& 
BARTSCH 1992). Die Wuchsleistung der Hainbuche ist im Allgemeinen erheblich geringer als die der Rotbuche und anderer Edellaubbäume (STAUFER 1996), was die hier durchgeführten Zuwachsuntersuchungen bestätigen.

Im Vergleich zu den untersuchten Eschen und Winterlinden bildete die Hainbuche signifikant größere Kronenschirmflächen aus (vgl. Abb. 4.7, Kap. 4.2.1), was durch die Ausprägung einer großen Schattenkrone bedingt ist. Die Hainbuche weist dementsprechend nicht die Tendenz auf, ihre Blattmasse auf den oberen Kronenbereich zu konzentrieren, wie es für die Esche nachgewiesen werden konnte. Auch scheint die Strategie, zunächst mit geringer Kronenausdehnung und starkem Höhenwachstum in die oberen Kronenbereiche vorzudringen und erst dann in die Breite zu wachsen, weniger ausgeprägt zu sein als bei der Esche und der Winterlinde. So wiesen Hainbuchen mit einem BHD von nur 20-30 cm schon Kronenflächen von 80-100 $\mathrm{m}^{2}$ auf. Auch die Tatsache, dass nicht die Bäume mit dem größten BHD die größten Schirmflächen aufwiesen, sondern Individuen mit Umfängen, die knapp über dem Durchschnitt $(40 \mathrm{~cm})$ lagen, belegt, dass die Kronenausdehnung bei dieser Baumart weniger stark an das Höhenwachstum gebunden ist als bei den anderen untersuchten Arten. Die im Kronenraum erhöhte Abbruchrate der seitlichen Triebe vor allem in Nachbarschaft zu Eschen kompensiert die Hainbuche teilweise mit einem Ausweichen in den unteren Kronenraum (vgl. Kap. 5.3.2). Zusammen mit der großen Schattentoleranz und der Anpassung an saisonale Trockenheit (HöLSCHER et al. 2005) sowie an Staunässe könnte die Hainbuche trotz ihrer relativen Konkurrenzschwäche eine geeignete Baumart für einen zweischichtigen Bestandesaufbau sein. Ob sie sich langfristig im Nationalpark Hainich ohne Pflegemaßnahmen halten können wird, erscheint hingegen fraglich.

\subsubsection{Tilia cordata}

Die Horizontalverbreitung der Winterlinde umfasst den größten Teil von West-, Mittel- und Osteuropa. Im Baltikum und in Ostpreußen liegen Schwerpunkte ihres Vorkommens. Sie ist hauptsächlich in der planaren, kollinen und submontanen Stufe zu finden, seltener in montanen Höhen (PIGOTT 1978, 1980, 1981; LEIBUNDGUT 1991). Die natürlichen Arealgrenzen lassen sich nach RÖHRIG \& BARTSCH (1992) kaum noch feststellen, da Linden schon im Mittelalter im Zuge der Mittelwaldwirtschaft vielfach gepflanzt worden sind. Im Verlauf des Umbaus der Waldwirtschaftsform von Mittel- und Niederwaldwirtschaft zur Kultivierung von Hochwäldern ist das Vorkommen der Winterlinde in Deutschland wieder zurückgegangen (BARTELS 1993). So ist sie heute in Mitteleuropa eine Mischbaumart in sommerwarmen Laub- und Laub-Nadelmischwäldern mit einer weit gespannten 
standörtlichen Amplitude (RÖHRIG \& BARTSCH 1992). Ihr Vorkommen beschränkt sich jedoch hauptsächlich auf trockene Eichen-Hainbuchenwälder, Ahorn-Eschen-Schluchtwälder auf Löss, Blockschuttwälder und nordalpine Linden-Steilhangwälder (ELLENBERG 1996), also auf Standorte, wo die Rotbuche durch Bodentrockenheit, Substratbewegung oder andere Standortfaktoren geschwächt wird. In Osteuropa, jenseits des Verbreitungsgebiets der Rotbuche, nimmt sie in Vergesellschaftung mit Hainbuche (Tilio-Carpinetum) sehr viel größeren Raum ein, was auf die, im Vergleich zur Rotbuche, sehr viel größere Toleranz gegenüber Trockenheit zurückzuführen ist (ELLENBERG 1996). Sie kann sowohl bei geringeren Jahresniederschlägen gedeihen, als auch stärkeren sommerlichen Trockenperioden standhalten (Ow 1948, PIGOTT 1991). Ihre gleichzeitig größere Winterhärte zeigt die bessere Angepasstheit dieser Art an kontinentale Klimabedingungen.

Die Winterlinde zeigt nach BöCKMANN (1990) in der Jugend ein etwas geringeres Höhenwachstum als Bergahorn und Esche, das dem der Rotbuche jedoch eindeutig überlegen ist (KRAMER 1988). Dieser Vorsprung wird allerdings von der Rotbuche nach den ersten 50 Lebensjahren eingeholt und mit 100 Jahren erbringt die Buche einen 30\% höheren Vorrat als die Linde, was die Nutzholzproduktion anbetrifft. Die Winterlinde erreicht auf geeigneten Standorten Höhen von 35-40 m und bildet im geschlossenen Bestand meist astreine Schäfte mit hoch angesetzten, walzenförmigen Kronen (NAMVAR \& SPETHMANN 1986). Diese neigen zu relativ weiten Ausladungen im Vergleich zur Rotbuche, was impliziert, dass die Winterlinde für einen guten Dickenzuwachs einen größeren Standraum als die Buche benötigt (ERTELT 1963).

Dem hohen Raum- und Lichtbedarf der Linde im Alter steht eine ausgeprägte Schattentoleranz in der Jungwuchsphase gegenüber (PIGOTT 1975). Die Linde weist eine hohe morphologische Flexibilität auf (HALlÉ et al. 1978). Potentiell sind alle Achsen zu sekundärer Aufrichtung fähig, was eine große Plastizität der Wuchsform mit sich bringt. Durch das zunächst horizontale Wachstum jedes Triebes sind Linden sehr effiziente Schattenerzeuger, was durch eine distiche Blattanordnung noch unterstützt wird.

Zudem ist die Baumart, vor allem bei seitlichem Lichteinfall auf den Stamm, sehr reiterationsfreudig, was die flexible Kronenausbildung ebenfalls unterstreicht.

Die im Rahmen dieser Arbeit untersuchten Linden zeigten als einzige Baumart keinen Zusammenhang zwischen Stammzuwachs und Bestandesklima. Zwar zeigen sich im Extremjahr 1976 auch bei der Winterlinde leichte Zuwachsrückgänge (Abb. 4.23, Kap. 4.2.2), jedoch sind diese sehr viel geringer als bei Hainbuche, Esche und Buche. Auch für das Jahr 2004 sind Zuwachsrückgänge zu verzeichnen. Im trockenen Spätsommer 2003 zeigte auch 
die Winterlinde deutliche Trockenstress-Reaktionen: Jeweils die kleinsten Schattenblätter jedes Zweiges verfärbten sich ab Ende Juli gelb und wurden ab Anfang August abgeworfen, die Blätter in der Sonnenkrone verfärbten sich nicht wie bei der Buche, sondern rollten sich ein, sodass nur noch die silbrige, reflektierende Unterseite der Blätter sichtbar war. Nach Einsetzen des Regens Ende August regenerierten sich die Bäume wieder im Gegensatz zu den Buchen im Bestand. Auch HöLSCHER et al. (2005) wiesen eine starke Reduktion der Saftflussdichte der Winterlinde in einer Trockenperiode 2001 nach. Im Vergleich zu Tagen mit ähnlichem VPD, aber feuchtem Boden war die Saftflussdichte um 44\% herabgesenkt. Damit zeigte die Winterlinde eine noch stärkere Reduktion als die Buche (39\% Reduktion). Die Autoren schließen aus diesen Messungen, dass die Reduktion der Saftflussdichte allein nicht als Maß für Trockenheitstoleranz herangezogen werden kann. Auch die absolute Höhe der Saftflussrate ist entscheidend, um den artspezifischen Wasserhaushalt zu charakterisieren. Diese ist bei der Winterlinde auch bei guter Wasserversorgung mit $86 \mathrm{~g} \mathrm{~cm}^{-2}$ pro Tag nur etwa halb so hoch wie die der Buche $\left(157 \mathrm{~g} \mathrm{~cm}^{-2}\right.$ pro Tag). Sie liegt aber in einer ähnlichen Größenordnung wie bei Hainbuche und Esche (77 bzw. $67 \mathrm{~g} \mathrm{~cm}^{-2}$ pro Tag). Eine geringe Saftflussrate auf Individuenebene erlaubt nach HÖLSCHER et al. (2005) eine weitere Standortamplitude, da die Art auch bei geringerem Wasserangebot ihre artspezifische Saftflussrate aufrechterhalten kann.

Auch die Winterlinde eignet sich wie die Hainbuche aufgrund ihrer Schattentoleranz als Mischbaumart (RÖHRIG 1966). Dies bestätigen auch physiologische Untersuchungen von KAZDA et al. (2000), die für Lindenblätter im Bestandesschatten auf einer Höhe von 15 m eine extrem hohe Stickstoff-Effizenz bei der Photosynthese feststellten, die die untergeordnete Stellung der Art in dem untersuchten Bestand zu einem gewissen Grad kompensieren konnte.

Die hier durchgeführten Untersuchungen der artspezifischen Kronenstrukturen zeigen jedoch deutlich, dass sich die Winterlinde am wenigsten von den hier untersuchten Baumarten für eine Mischung mit Buche eignet, da sich die Kronen der beiden Arten auf genau der gleichen Bestandeshöhe ausbreiten und es so zu einer sehr intensiven Konkurrenzsituation kommt (vgl. Abb. 5.2, Kap. 5.2.2). 


\subsubsection{Schlussfolgerungen}

Wie schon in Kapitel 5.1 erwähnt, ist die gute vegetative Verjüngung der Winterlinde ein Faktor, der es wahrscheinlich macht, dass sich die Winterlinde auch weiterhin auf den lindenreichen Flächen 1 und 2 im Lindig halten können wird. Eine weitere Ausbreitung der Art im Nationalpark könnte nur durch eine störungsbedingte Schwächung der Buche zustande kommen.

So wird für die weitere Waldentwicklung im Nationalpark Hainich die Intensität und Häufigkeit von Trockenperioden in der Vegetationsperiode und von Sturmereignissen über die Waldstruktur und Baumartenzusammensetzung entscheiden. Wie verschiedene große Windwurfflächen im Hainich zeigen, werden auch größere Flächen gleich wieder von den angrenzenden spätsukzessionalen Baumarten besiedelt. Im Einklang mit den Untersuchungen von SCHMIDT (1998) bestätigt sich das von REMMERT (1991) entworfene Mosaik-ZyklusKonzept nicht, nach dem sich in offenen Bestandesflächen erst wieder frühsukzessionale Arten ansiedeln müssten. So scheinen extreme Windereignisse stärker die Struktur der Bestände zu beeinflussen als die Artenzusammensetzung. Letztere scheint langfristig vor allem von Trockenperioden beeinflussbar. Bei einer geringen Störungsintensität muss auf den buchenreichen Flächen von einer weiter fortschreitenden Entmischung und einer damit verbundenen starken Buchendominanz ausgegangen werden (WOHLGEMUTH et al. 2002, MUND 2005).

Auch auf den Flächen im Lindig werden konkurrenzschwächere Arten wie die Hainbuche, aber auch die Eiche und andere hier nicht untersuchte Laubholzarten vermutlich zurückgehen. Eine vollständige Umwandlung in einen Buchenreinbestand erscheint aber auch unter den jetzigen Bedingungen aufgrund der Abundanz der zur Zeit etablierten anderen Arten und dem unausgeglichenen Wasserhaushalt am Ostrand des Hainich als unwahrscheinlich. Stärkere und häufigere Trockenperioden werden im Osthainich zu einer Dominanzreduktion der Buche führen. Als Konsequenz ergibt sich eine erhöhte Diversität der Baumartenzusammensetzung, da auf diesem Standort nicht davon auszugehen ist, dass die Buche von einer Art ersetzt werden wird, sondern eine Vielzahl von Arten - darunter auch die Esche, Hainbuche und Winterlinde - um den entstandenen Raum konkurrieren werden (WOHLGEMUTH et al. 2002). Aus den hier durchgeführten Untersuchungen zur Verjüngung, zur Kronenstruktur und -interaktion sowie zum Zuwachsverhalten kann abgeleitet werden, dass dabei die Chancen auf eine langfristige Etablierung bzw. auf eine weitere Ausbreitung der hier untersuchten Arten von der Hainbuche über die Winterlinde hin zur Esche zunimmt. 


\section{Zusammenfassung}

Im Rahmen dieser Studie wurde die dreidimensionale Bestandesstruktur naturnaher artenreicher Mischbestände auf vier Untersuchungsflächen von je 0.64 ha Größe am Ostrand des Nationalparks Hainich (Thüringen) untersucht. Vor dem Hintergrund, dass es sich bei den untersuchten Beständen um Laubmischwälder innerhalb des natürlichen Buchenareals handelt, wurde der Frage nachgegangen, ob die derzeitige Bestandesstruktur Rückschlüsse darüber zulässt, ob es mittelfristig zu einer Entmischung der artenreichen Wälder kommen wird, indem die Buche ihre natürliche Vorherrschaft zurückgewinnt.

Neben einer vollständigen Kartierung der Kronenflächen, Verjüngungsaufnahmen und Bestimmung des artspezifischen Totholzvolumens erfolgten vertiefende Untersuchungen zur horizontalen und vertikalen Besetzung des Kronenraums, der Nachbarschaftsinteraktion im Kronenraum (Trieblängenrückmessungen, Strahlungsmessungen und Aststellungsanalyse) und zum Wuchsverhalten (dendroökologische Jahrringanalyse) der Rotbuche, sowie der drei weiteren, häufig vertretenen Baumarten Esche, Hainbuche und Winterlinde.

Fläche 1 und 2 waren in der Baumschicht stark von Linden dominiert (47\% bzw. 57\% der Bäume $>7 \mathrm{~cm}$ BHD). Die Verjüngung setzte sich zum größten Teil aus Esche (57\% bzw. 65\%) und Ahorn zusammen. Winterlinden verjüngten sich meist vegetativ durch Stockausschläge, Buchenjungpflanzen waren kaum vertreten. Am Totholzvorkommen hatte die Buche jedoch auf Fläche 2 mit 26\% einen überproportional hohen Anteil (Anteil an der Baumschicht: 7\%). Auf Fläche 3 waren die Anteile an der Baumschicht wie am Totholz ausgeglichener, doch auch hier dominierten Esche und Ahorn die Verjüngung. Dies galt auch für die von Buchen dominierte Fläche 4 (52\%). Auch hier war der Anteil der Buchenverjüngung mit knapp 6\% sehr gering. Die räumliche Verteilung der Jungpflanzen zeigte sich unabhängig von der Verteilung der Altbäume der gleichen Art und auch von der relativen Beleuchtungsstärke am Waldboden.

Hainbuchen wiesen mit $100 \mathrm{~m}^{2}$ die signifikant größten Kronenflächen auf. Im Vergleich der Lichtkronenanteile (Hainbuche: $50 \mathrm{~m}^{2}$ ) zeigten sich jedoch keine signifikanten Unterschiede zwischen den Arten. Außer der Kronenfläche wurde auch die Höhe bestimmt, auf der die Baumarten ihre maximale Kronenbreite erreichen. Eschen breiten ihre Kronen deutlich höher und Hainbuchen deutlich tiefer im Bestand aus als Buchen und Winterlinden. Die Unterschiede waren hochsignifikant. Nicht zuletzt dadurch ergaben sich zwischen benachbarten Hainbuchen und Eschen die größten Überlappungsbereiche der Kronen, wobei die Überlappungsfläche bis zu 45\% der Gesamtkronenfläche einnehmen konnte. 
Bei der Untersuchung der Kroneninteraktion durch Trieblängenrückmessungen ergaben sich signifikante Unterschiede zwischen den Längen und Umfängen der vermessenen Zweige im Wipfel und dem seitlichen Kronenbereich, der im direkten Kontakt zu angrenzenden Baumkronen stand. Signifikante Unterschiede des Trieblängen- und Dickenwachstums innerhalb einer Art je nach Artzugehörigkeit des Nachbarbaumes zeigten sich nicht. Unterschiede zwischen den verschiedenen Nachbarschaftskonstellationen zeigten sich jedoch bei der Häufigkeit der Leittriebunterbrechungen durch Astabbrüche. Hier zeigte sich die Hainbuche von benachbarten Eschen deutlich stärker geschädigt als von anderen Nachbararten. Richtungsänderungen des Astwachstums waren bei Hainbuche und Winterlinde häufiger $\mathrm{zu}$ beobachten als bei Esche und Buche. In einer multiplen linearen Regression wurde geprüft, wie stark mögliche Einflussfaktoren das Trieblängenwachstum bestimmen. Die relative Beleuchtungsstärke, die jeweils an der Triebspitze gemessen wurde, erwies sich nur für Buche als signifikante Einflussgröße. Für alle Baumarten spielte die Position des Triebes innerhalb der Krone eine Rolle. Winterlinden und Hainbuchentrieblängen zeigten eine deutliche Abhängigkeit von der Artzugehörigkeit der Nachbarbäume. Auch die Häufigkeit der Astabbrüche, die durch Kronenkollisionen hervorgerufen werden, hatten auf die Triebentwicklung von Buchen und Hainbuchen einen signifikanten Einfluss. Die Ergebnisse lassen den Schluss zu, dass die mechanische Kronenkollision bei Wind eine bislang deutlich unterschätzte Form der Interaktion zwischen Altbäumen im geschlossenen Bestand ist.

In der vergleichenden dendroökologischen Untersuchung zeigte die Buche die stärkste Korrelation von Jahrringbreite und Niederschlag der Vegetationsperiode $(\mathrm{r}=0.52)$. Ein signifikanter Zusammenhang zwischen Klima und Ringbreite zeigte sich auch für die Esche $(\mathrm{r}=0.43)$ und die Hainbuche $(\mathrm{r}=0.32)$, jedoch nicht für die Winterlinde. Diese Art zeigte zudem die geringste Sensitivität sowie die höchste Autokorrelation der Ringbreiten. Über die letzten 50 Jahre wies die Buche im Mittel das stärkste Dickenwachstum auf, dieses ist aber in den letzten Jahrzehnten rückläufig und wird seit ca. 1990 von der Esche übertroffen.

Unter den derzeitigen Bedingungen scheinen Winterlinden und vor allem Eschen ihre Anteile auf den beiden von Linden dominierten Flächen zumindest behaupten, wenn nicht sogar ausbauen zu können, da die Buche sich dort kaum verjüngt und die Altbäume nur eingeschränkt vital sind. Die Hainbuche erweist sich am stärksten von der interspezifischen Konkurrenz betroffen. Ob die Buche ihre Dominanz auf der buchenreichen Fläche 4 erhalten kann, wird maßgeblich von der Häufigkeit und Intensität von sommerlichen Trockenperioden abhängen, unter denen die Buche auch heute schon am stärksten zu leiden hat. 


\section{Literaturverzeichnis}

Aarssen, L. 1995. Hypotheses for the evolution of apical dominance in plants: Implications for the interpretation of overcompensation. Oikos 74:149-156.

Albrecht, L. 1991. Die Bedeutung des toten Holzes im Wald. Forstwissenschaftliches Centralblatt 110:106-113.

Ammer, C. 2003. Growth and biomass partitioning of Fagus sylvatica L. and Quercus robur L. seedlings in response to shading and small changes in the R/FR-ratio of radiation. Annals of Forest Science 60:163-171.

Anderson, M. C. 1963. Studies of the woodland climate. The photographic computation of light conditions. Journal of Ecology 52:27-41.

Andersson, M., Y. Birot, and R. Päivinen. 2004. Towards the Sustainable Use of Europe's Forests - Forest Ecosystem and Landscape Research: Scientific Challenges and Opportunities., 1 edition. European Forest Institute, Saarijärvi, Finnland.

Aphalo, P. J., C. L. Ballaré, and A. L. Scopel. 1999. Plant-plant signalling, the shadeavoidance response and competition. Journal of Experimental Botany 50:1629-1634.

Appt, M., C. Reich, and A. Scheuermann. 1983. Höhenzuwachsentwicklung bei Immissionsschäden von Fichte und Tanne. Allgemeine Forstzeitschrift 38:368-370.

Asthalter, K. 1984. Trockenperioden und Waldschäden aus forstgeschichtlicher und standortskundlicher Sicht. Allgemeine Forstzeitschrift 39:549-551.

Attorini, M. R., M. Galli, T. Nanni, and P. Povinec. 1989. A cyclogram analysis of the Bratislava ${ }^{14} \mathrm{C}$ tree-ring record during the last century. Radiocarbon 31:839-845.

Bachmann, M. 2002. Indizes zur Erfassung der Konkurrenz von Einzelbäumen. Methodische Untersuchung in Bergmischwäldern. Forstliche Forschungsberichte München, München.

Baillie, M. G. L., and J. R. Pilcher. 1973. A simple crossdating program for tree-ring research. Tree-Ring Bulletin 33:7-14.

Ballaré, C. L., A. L. Scopel, M. L. Roush, and S. R. Radosevich. 1995. How Plants Find Light in Patchy Canopies. A Comparison between Wild-Type and Phytochrome-B-Deficient Mutant Plants of Cucumber. Functional Ecology 9:859-868.

Barnes, P. W., W. Beyschlag, R. Ryel, S. D. Flint, and M. M. Caldwell. 1990. Plant competition for light analysed with a multispecies canopy model. III. Influence of canopy structure in mixtures and monocultures of wheat and wild oat. Oecologia 82:560-566.

Bartels, H. 1993. Gehölzkunde. Einführung in die Dendrologie. Ulmer, Stuttgart.

Bechtold, W. A., M. E. Mielke, and S. J. Zarnoch. 2002. Comparison of field methods and models to estimate mean crown diameter. NJAF 19:177-182. 
Bella, I. E. 1971. A new competition model for individual trees. Forest Science 17:364-372.

Berli, S., and F. H. Schweingruber. 1992. Auswirkungen des Waldbrandes vom 10. bis 14. Mai 1965 am Monte San Giorgio, Tessin, Schweiz: Eine dendroökologische Studie. Schweizerische Zeitschrift für das Forstwesen 143:957-982.

Bobiec, A. 2002. Living stands and dead wood in the Bialowieza forest: suggestions for restoration management. Forest Ecology and Management 165:125-140.

Bonn, S. 1998. Dendroökologische Untersuchung der Konkurrenzdynamik in Buchen/EichenMischbeständen und zu erwartende Modifikationen durch Klimaänderungen. Fachrichtung Forstwissenschaften der TU Dresden, Tharandt, Dresden.

Bossel, H. 1991. Modelling forest dynamics: moving from description to explanation. Forest Ecology and Management 42:129-142.

Bouriaud, O., N. Bréda, G. Le Moguèdec, and G. Nepveu. 2004. Modelling variability of wood density in beech as affected by ring age, radial growth and climate. Trees 18:264-276.

Böckmann, T. 1990. Wachstum und Ertrag der Winterlinde (Tilia cordata Mill.) in Niedersachsen und Nordhessen. Dissertationsschrift, Universität Göttingen.

Braun, S., and W. Flückiger. 1987. Untersuchungen an Gipfeltrieben von Buche (Fagus sylvatica L.). Botanica Helvetica 97:61-73.

Broadmeadow, M., and S. B. Jackson. 2000. Growth responses of Quercus petraea, Fraxinus excelsior and Pinus sylvestris to elevated Carbon Dioxide, Ozone and Water supply. The New Phytologist 146:437-451.

Buck-Sorlin, G. H., and A. D. Bell. 2000. Crown architecture in Quercus petraea and Q. robur: the fate of buds and shoots in relation to age, position and environmental pertubation. Forestry 73:331-349.

Buck-Sorlin, G. H., and A. D. Bell. 2000. Models of crown architecture in Quercus petraea and $Q$. robur: shoot length and bud numbers. Forestry 73:1-19.

Burgdorf, N. 2004. Waldstruktur und Bodeneigenschaften in unterschiedlich artenreichen Laubwaldbeständen im Hainich, Thüringen. Diplomarbeit angefertigt in der Abteilung Ökologie und Ökosystemforschung am Albrecht-von-Haller-Insitut für Pflanzenwissenschaften der Universität Göttingen.

Calton, N., and J. Fletcher. 1978. A comparison of the ring width of oak and ash. Pages 184186 in J. Fletcher editor. Dendochronology in Europe. University Press, Oxford.

Canham, C. D., and D. H. Burbank. 1994. Causes and consequences of resource heterogeneity in forests: interspecific variation in light transmission by canopy trees. Canadian Journal of Forest Reseach 24:337-349.

Catovsky, S., and F. A. Bazzaz. 2002. Feedbacks between canopy composition and seedling regeneration in mixed conifer broad-leaved forests. Oikos 98:403-420. 
Cescatti, A., and E. Piutti. 1998. Silvicultural alternatives, competition regime and sensitivity to climate in a European beech forest. Forest Ecology and Management 102:213-223.

Chason, J. W., D. D. Baldocchi, and M. A. Huston. 1991. A comparison of direct and indirect methods for estimating forest canopy leaf area. Agricult.Forest Meteorol. 57:107-125.

Choi, J., C. G. Lorimer, J. Vanderwerker, W. C. Cole, and G. L. Martin. 2001. A crown model for simulating long-term stand and gap dynamics in northern hardwood forests. Forest Ecology and Management 152:235-258.

Cieslar, A. 1907. Einige Beziehungen zwischen Holzzuwachs und Witterung. Centralblatt für das gesamte Forstwesen 33:289-311.

Claessens, H., D. Pauwels, A. Thibaut, and J. Rondeux. 1999. Site index curves and autecology of ash, sycamore and cherry in Wallonia (Southern Belgium). Forestry 72:171-182.

Coomes, D., and P. J. Grubb. 2000. Impacts of root competition in forests and woodlands: a theoretical framework and review of experiments. Ecological Monographs 70:171207.

Crookshanks, M., G. Taylor, and M. Broadmeadow. 1998. Elevated $\mathrm{CO}_{2}$ and tree root growth: contrasting responses in Fraxinus excelsior, Quercus petraea and Pinus sylvestris. The New Phytologist 138:241-250.

Degenhardt, A. 1994. Verfahren zur Charakterisierung der Konkurrenzverhältnisse in Mischbeständen. Beiträge für Forstwirtschaft und Landschaftsökologie 28:87-89.

Dittmar, C., and W. Zech. 1994. Dendroökologische und ernährungskundliche Untersuchungen an gesunden und kranken Buchen (Fagus sylvatica L.) der nordböhmischen Mittelgebirge. Dendrochronologia 12:45-58.

Dittmar, C., W. Zech, and W. Elling. 2003. Growth variations of Common beech (Fagus sylvatica L.) under different climatic and environmental conditions in Europe - a dendroecological study. Forest Ecology and Management 173:63-78.

Döbbele,r H., Albert M., Schmidt M. \& Nagel J. 2003. BWINPro - Programm zur Bestandesanalyse und Prognose. Handbuch zur Version 6.2. Abteilung Waldwachstum Niedersächsische Forstliche Versuchsanstalt. Göttingen.

Dufrêne, E., and N. Bréda. 1995. Estimation of deciduous forest leaf area index using direct and indirect methods. Oecologia 104:156-162.

Duquesnay, A., N. Bréda, M. Stievenard, and J. L. Dupouy. 1998. Changes of tree-ring $\delta^{13} \mathrm{C}$ and water-use efficiency of beech (Fagus sylvatica L.) in north-eastern France during the past century. Plant, Cell and Environment 21:565-572.

Eckstein, D., and J. Bauch. 1969. Beitrag zur Rationalisierung eines dendrochronologischen Verfahrens und zur Analyse seiner Aussagesicherheit. Forstwissenschaftliches Centralblatt 88:230-250. 
Eckstein, D., K. Richter, R. W. Aniol, and F. Quiehl. 1984. Dendroklimatologische Untersuchungen zum Buchensterben im südwestlichen Vogelsberg. Forstwissenschaftliches Centralblatt 103:274-289.

Ellenber, H. 1996. Vegetation Mitteleuropas mit den Alpen in ökologischer, dynamischer und historischer Sicht. Ulmer, Stuttgart.

Ertelt, W. 1963. Über die Wachstumsentwicklung der Linde. Archiv für das Forstwesen 12:1152-1158.

Ewald, A. 2001. Pflanzensoziologische Untersuchungen im Nationalpark Hainich. Nationalparkverwaltung Hainich, Bad Langensalza.

Fabian, P., and A. Menzel. 1998. Wie sehen die Wälder von morgen aus - aus der Sicht eines Klimatologen. Forstwissenschaftliches Centralblatt 117:339-354.

Falinski, J. B. 1986. Vegetation dynamics in temperate lowland primeval forests. Ecological studies in Bialowieza Forest. Junk Publishers, Dordrecht.

Felbermeier, B. 1994. Die klimatische Belastbarkeit der Buche. Forstwissenschaftliches Centralblatt 113:152-174.

Fischer, H., and W.-D. Rommel. 1989. Jahrringbreiten und Höhentrieblängen von Buchen mit unterschiedlicher Belaubungsdichte in Baden-Württemberg. Allgemeine Forstzeitschrift 11:264-265.

Flesch, T. K., and J. D. Wilson. 1999. Wind and remnant tree sway in forest cutblocks. II. Relating measured tree sway to wind statistics . Agricultural and Forest Meteorology 93:243-258.

Franco, M. 1986. The influence of neighbours on the growth of modular organisms with an example from trees. Philosophical Transactions of the Royal Society - Biological Science 313:209-225.

Frech, A., C. Leuschner, M. Hagemeier, and D. Hölscher. 2003. Nachbarschaftsbezogene Analyse der Kronenraumbesetzung von Esche, Hainbuche und Winterlinde in einem artenreichen Laubmischwald (Nationalpark Hainich, Thüringen). Forstwissenschaftliches Centralblatt 122:22-35.

Frelich, L. E., S. Sugita, P. B. Reich, M. B. Davis, and S. K. Friedman. 1998. Neighbourhood effects in forests: implications for within-stand patch structure. Journal of Ecology 86:149-161.

Fritts, H. C. 1976. Tree ring and climate. Academic press, London, New York, San Francisco.

Gadow, K. v., G. Y. Hui, and M. Albert. 1998. Das Winkelmaß - ein Strukturparameter zur Beschreibung der Individualverteilung in Waldbeständen. Centralblatt für das gesamte Forstwesen 115:1-10.

Gatzer, G., C. D. Canham, U. Dieckmann, A. Fischer, Y. Iwasa, M. Law, M. Lexer, H. Sandmann, T. A. Spies, E. Splechtna, and J. Szwagrzyk. 2004. Spatio-temporal development of forests - current trends in field methods and models. Oikos 107:3-15. 
Gia, T. D. 1927. Beitrag zur Kenntnis der Schattenfestigkeit verschiedener Holzarten im 1. Lebensjahr. Forstwissenschaftliches Centralblatt. 49:386-397.

Gleissner P. 1998. Das Verzweigungsmuster ausgewählter Laubbaumarten und seine Veränderung durch nicht-pathogene Schädigungen. Palmarum Hortus Francofurtensis Wissenschaftliche Berichte. Frankfurt.

Gohrbandt, A. 2005. Betrachtungen zum Wildtiermanagement im Nationalpark Hainich. Diplomarbeit, Fachhochschule Eberswalde.

Grace, J. B. 1990. On the relationship between plant traits and competitive ability. Pages 5166 in J. B. Grace, and D. Tilman editors. Perspectives on plant competition. Academic Press, San Diego, California.

Grace, S. L., and W. J. Platt. 2002. Neighborhood effects on juveniles in an old-growth stand of longleaf pine, Pinus palustris. Oikos 72:99-105.

Grams, T. E., A. R. Kozovits, I. M. Reiter, J. B. Winkler, M. Sommerkorn, H. Blaschke, K.H. Häberle, and R. Matyssek. 2002. Quantifying competitiveness in woody plants. Plant Biology 4:153-158.

Grime, J. P. 1979. Plant Strategies and Vegetation Processes. J. Wiley, Chichester.

Grissino-Mayer, H.D. \& Kaenell Dobbertin M. 2003. Dendrochronology Species Database. Names of tree and shrub species for which tree rings have been analysed in the published literature. http://www01.wsl.ch/species/about.html.

Grosser, D., and E. Graessle. 1976. Die in der Tafelmalerei und Bildschnitzerei verwendeten Holzarten und ihre Bestimmung nach mikroskopischen Merkmalen. Teil II. Europäische Laubhölzer, Fortsetzung. Restauro 4:232-252.

Grote, R., and H. Pretzsch. 2002. A Model for individual tree development based on physiological processes. Plant Biology 4:167-180.

Guericke, M. 2001. Untersuchungen zur Wuchsdynamik von Mischbeständen aus Buche und Europäischer Lärche (Larix decidua, Mill.) als Grundlage für ein abstandsabhängiges Einzelbaumwachstumsmodell. Dissertationsschrift, Universität Göttingen.

Guyan, W. U. 1977. Archäologie und Dendrochronologie der jungsteinzeitlichen Moordörfer Thayngen-Weier. Pages 126-142 in B. Frenzel editor. Dendrochronologie und postglaziale Klimaschwankungen in Europa. Franz Steiner Verlag, Wiesbaden.

Hagemeier, M. 2002. Funktionale Kronenarchitektur mitteleuropäischer Baumarten am Beispiel von Hängebirke, Waldkiefer, Traubeneiche, Hainbuche, Winterlinde und Rotbuche., Dissertationes Botanicae edition. Cramer, Berlin, Stuttgart.

Hallé, F., R. A. A. Oldeman, and P. B. Tomlinson. 1978. Tropical trees and forests: an architectural analysis. Springer, Berlin.

Harmer, R. 1999. Survival and new shoot production by artificially browsed seedlings of ash, beech, oak and sycamore grown under different levels of shade. Forest Ecology and Management 116:38-50. 
Harmer, R. 2001. The effect of plant competition and simulated summer browsing by deer on tree regeneration. Journal of Applied Ecology 38:1094-1103.

Harper, J. L. 1981. The concept of population in modular organisms. Pages 53-77 in R. M. May editor. Theoretical ecology: principles and applications. Blackwell Scientific Publications, Oxford.

Hättenschwiler, S. 2001. Tree seedling growth in natural deep shade: functional traits related to interspecific variation in response to elevated $\mathrm{CO}_{2}$. Oecologia 129:31-42.

Hegyi, F. 1974. A simulation model for managing jack-pine stands. Pages 74-90 in J. Fries editor. Growth models for tree and stand simulation. Royal College of Forest, Stockholm.

Henriksson, J. 2001. Differential shading of branches or whole trees: survival, growth, and reproduction. Oecologia 126:482-486.

Hertel, D., H. Coners, A. Muhs, F. Schipka, and J. Strobel. 2004. Zur Trockenheitsgrenze der Buche in Mittel- und Ostdeutschland: Eine ökosystemare Transektstudie an unterschiedlich wasser- und nährstoffversorgten Buchen-Altbeständen. Berichte des Landesamts für Umweltschutz Sachsen-Anhalt, Sonderheft 2:28-37.

Hérault, B., D. Thoen, and O. Honnay. 2004. Assessing the potential of natural woody species regeneration for the conversion of Norway spruce plantations on alluvial soils. Annals of Forest Science 61:711-719.

Hiekel, W., Fritzlar F., Nöllert A. \& Westhus W. 2004. Die Naturräume Thüringens. Naturschutzreport 21:1-383.

Hix, D. M., and C. G. Lorimer. 1990. Growth-competition relationships in young hardwood stands on two contrasting sites in southwestern Wisconsin. Forest Science 36:10321049 .

Holbrook, N. M., and F. E. Putz. 1989. Influence of neighbors on tree form: effects of lateral shade and prevention of sway on the allometry of Liquidambar styracifula (sweet gum). American Journal of Botany 76:1740-1749.

Holbrook, N. M., and C. P. Lund. 1995. Photosynthesis in forest canopies. Pages 411-430 in M. D. Lowman, and N. M. Nadkarni editors. Forest Canopies. Academic Press, San Diego; London.

Hollstein, E. 1980. Mitteleuropäische Eichenchronologie. Trierer Grabungen und Forschungen 11:1-273.

Hoshino, D., N. Nishimura, and S. Yamamoto. 2001. Age, size structure and spatial pattern of major tree species in an old-growth Chamaecyparis obtusa forest, Central Japan. Forest Ecology and Management 152:31-43.

Höfler, K., and W. Elling. 1966. Der Jahrringbau von Linden des Dörnhöfferschen Parkes in Bayreuth. Berichte der Deutschen Botanischen Gesellschaft 79:3-6. 
Hölscher, D. 2003. Leaf traits and photosynthetic parameters of saplings and adult trees of coexisting species in a temperate broad-leaved forest. Basic and Applied Ecology 5:163172.

Hölscher, D., O. Koch, S. Korn, and C. Leuschner. 2005. Sap flux of five co-occuring tree species in a temperate broad-leaved forest during seasonal soil drought. Trees 19:628637.

Huldén, E. 1941. Studien über Fraxinus excelsior L. Acta Botanica Fennica 28:1-239.

Huss, J. 1987. Mischwald zwischen Wunsch und Wirklichkeit. Forstwissenschaftliches Centralblatt 106:114-132.

IPCC Working Group II. Climate Change 2001: Impacts, Adaptation and Vulnerability. http//:www.ipcc.ch.

Iseli, M., and F. H. Schweingruber. 1989. Sichtbarmachen von Jahrringen für dendrochronologische Untersuchungen. Dendrochronologia 7:145-157.

Johnson, W. C. 1988. Estimating dispersibility of Acer, Fraxinus and Tilia in fragmented landscapes from patterns of seedling establishment. Landscape Ecology 1:175-187.

Jonckheere, I., S. Fleck, K. Nackaerts, B. Muys, P. Coppin, M. Weiss, and F. Baret. 2004. Review of methods for in situ leaf area index determination - Part I. Theories, sensors and hemispherical photography. Agricultural and Forest Meteorology 121:19-35.

Kazda, M., J. Salzer, and I. Reiter. 2000. Photosynthetic capacity in relation to nitrogen in the canopy of a Quercus robur, Fraxinus angustifolia and Tilia cordata flood plain forest. Tree Physiology 20:1029-1057.

Kelty, J. M. 1989. Productivity of New England hemlock/hardwood stands as affected by species composition and canopy structure. Forest Ecology and Management 28:237257.

Kenk, G. K. 1992. Silviculture of mixed-species stands in Germany. Pages 53-64 in M. G. R. Cannell, D. C. Malcom, and P. A. Robertson editors. The ecology of mixed-species stands of trees.

Kennel, E. 1984. Untersuchung des Höhenzuwachses von Fichten mit Waldkrankheitssymptomen im Bereich der OFD Augsburg. Allgemeine Forstzeitschrift 39:465-469.

Kerr, G., and C. Cahalan. 2004. A review of site factors affecting the early growth of ash (Fraxinus excelsior L.). Forest Ecology and Management 188:225-234.

Kikuzawa, K., and K. Umeki. 1996. Effect of canopy structure on degree of asymmetry of competition in two forest stands in northern Japan. Annals of Botany 77:565-571.

Kirby, K. J., C. M. Reid, R. C. Thomas, and F. B. Goldsmith. 1998. Preliminary estimates of fallen dead wood and standing dead trees in managed and unmanaged forests in Britain. Journal of Applied Ecology 35:148-155. 
Kirschbaum, M. U. F., A. Fischlin, M. G. R. Cannell, R. V. O. Cruz, and W. Galinski. 1996. Climate change impacts on forests. Pages 95-129 in R. T. Watson, M. C. Zinyowera, and R. H. Moss editors. Camridge University Press, Cambridge, United Kingdom and New York, NY, USA.

Klaus, S. \& Reisinger E. 1995. Der Hainich: Ein Weltnaturerbe. Landschaftspflege und Naturschutz 32 (Sonderheft):1-33.

Knoerzer, D. 2002. Der Einfluß der Baumartenzusammensetzung des Hauptbestands auf die Baumartenverjüngung. Forstwissenschaftliches Centralblatt 121:283-300.

Koike, F. 1986. Canopy dynamics estimated from shoot morphology in an evergreen broadleaved forest. Oecologia 70:348-350.

Koike, F. 1989. Foliage-crown development and interaction in Quercus gilva and Quercus acuta. Journal of Ecology 77:92-111.

Korn, S. 2004. Experimentelle Untersuchungen der Wasseraufnahme und der hydraulischen Eigenschaften des Wurzelsystems von sechs heimischen Laubbaumarten. Dissertationsschrift, Georg-August-Universität Göttingen.

Kramer, H. 1980. Kurzfristige Zuwachsreaktionen bei Buche in Abhängigkeit von der Witterung und verschiedenen Baummerkmalen. Allgemeine Forst und Jagd-Zeitung 153:57-67.

Kramer, H., and A. Akca. 1987. Leitfaden für Dendrometrie und Bestandesstruktur. J.D. Sauerländer's Verlag, Frankfurt a.M.

Küppers, M. 1985. Carbon relations and competition between woody species in a Central European hedgerow. IV. Growth form and partitioning. Oecologia 66:343-352.

Küppers, M. 1987. Hecken. Ein Modellfall für die Partnerschaft von Physiologie und Morphologie bei der pflanzlichen Produktion in Konkurrenzsituationen. Naturwissenschaften 74:536-547.

Küster, H. 1996. Die Stellung der Hainbuche in der Vegetationsgeschichte. in B. L. f. W. u. Forstwirtschaft (ed.). Beiträge zur Hainbuche. Bayrische Landesanstalt für Wald und Forstwirtschaft, Freising:10-16

Küster, H. 1997. The role of farming in the post-glacial expansion of beech and hornbeam in oak woodlands of Central Europe. The Holocene 7:32-41.

Lang, G. 1994. Quartäre Vegetationsgeschichte Europas. Methoden und Ergebnisse. Jena, Stuttgart.

Le Goff, N., and J.-M. Ottorini. 1996. Leaf development and stem growth of ash (Fraxinus excelsior) as affected by tree competitive status. Journal of Applied Ecology 33:793802.

Le Goff, N., and J. M. Ottorini. 1993. Thinning and climate effects on growth of beech (Fagus sylvatica L.) in experimental stands. Forest Ecology and Management 62:1-14. 
Lebourgeois, F., N. Bréda, E. Ulrich, and A. Granier. 2005. Climate-tree-growth relationships of European beech (Fagus sylvatica L.) in the French Permanent Plot Network (RENECOFOR). Trees 19:385-401.

Leibundgut, H. 1991. Unsere Waldbäume: Eigenschaften und Leben., 2 edition. Haupt, Bern; Stuttgart.

Leuschner, C., K. Backes, D. Hertel, F. Schipka, U. Schmitt, O. Terborg, and M. Runge. 2001. Drought responses at leaf, stem and fine root level of competitive Fagus sylvatica L. and Quercus petraea (Matt. Liebl.) trees in dry and wet years. Forest Ecology and Management 149:33-46.

Leuschner, C. 1998. Mechanismen der Konkurrenzüberlegenheit der Rotbuche. Berichte der Reinh.-Tüxen-Ges. 10:5-18.

Leuschner, C., and F. Schipka. 2004. Vorstudie Klimawandel und Naturschutz in Deutschland. Abschlussbericht eines F+E-Vorhabens zur Erstellung einer Literaturstudie. Bonn - Bad Godesberg.

Lewis, S. L., and E. V. Tanner. 2000. Effects of above- and belowground competition on growth and survival of rain forest tree seedlings. Ecology 81:2525-2538.

Lorimer, C. 1983. Tests of age-independent competition indices for individual trees in natural hardwood stands. Forest Ecology and Management 6:343-360.

Lowman, M. D., and P. K. Wittman. 1996. Forest canopies: Methods, hypotheses, and future directions. Ann.Rev.Ecol.Syst. 27:55-81.

Lyr, H., G. Hoffmann, and W. Engel. 1964. Über den Einfluß unterschiedlicher Beschattung auf die Stoffproduktion von Jungpflanzen einiger Waldbäume. II. Mitteilung. Flora 155:305-330.

Marigo, G., J.-P. Peltier, and J. Girel. 2000. Success in the demographic expansion of Fraxinus excelsior L. Trees 15:1-13.

Maurer, E. 1965. Waldbauliche und holzkundliche Untersuchungen an Eschen aus dem Allgäu. Forst und Holz 43:162-188.

Medlyn, B. E., F. W. Badeck, D. G. G. De Pury, C. V. M. Barton, M. Broadmeadow, R. Creulemans, P. De Angelis, M. Forstreuter, M. E. Jach, S. Kellomäki, E. Laitat, M. Marek, S. Philippot, A. Rey, and P. G. Jarvis. 1999. Effects of elevated $\mathrm{CO}_{2}$ on photosynthesis in European forest species: a meta-analysis of model parameters. Plant, Cell and Environment 22:1475-1495.

Meinen, C. 2004. Der Einfluss der Baumartenzusammensetzung auf die Verjüngung von Carpinus betulus, Fagus sylvatica, Fraxinus excelsior und Tilia cordata in artenreichen Mischwaldbeständen im Nationalpark Hainich. Diplomarbeit angefertigt in der Abteilung Ökologie und Ökosystemforschung am Albrecht-von-Haller Institut für Pflanzenwissenschaften Universität Göttingen.

Meldahl, R. S., N. Pederson, J. S. Kush, and J. M. Varner. 1999. Dendroecological investigations of climate and competitive effects on longleaf pine growth. Pages 265- 
285 in R. Wimmer, and R. E. Vetter editors. Tree-ring analysis: biological, methodological, and environmental aspects. University Press, Cambidge.

Meyer, P., V. Tabaku, and B. v. Lüpke. 2003. Die Struktur albanischer Rotbuchen-Urwälder Ableitungen für eine naturnahe Buchenwirtschaft. Forstwissenschaftliches Centralblatt 122:47-58.

Meyer, P., Ackermann J., Balcar P., Boddenberg J., Detsch R., Förster B., Fuchs H., Hoffmann B., Keitel W., Kölbel M., Köthke C., Koss H., Unkrig W., Weber J. \& Willig J. 2000. Untersuchung der Waldstruktur und ihrer Dynamik in Naturwaldreservaten: methodische Empfehlungen. Erarbeitet im Auftrag des Arbeitskreises Naturwälder in der Bund-Länder-Arbeitsgemeinschaft Forsteinrichtung, Eching.

Modrý, M., D. Hubený, and K. Rejsek. 2004. Differential response of naturally regenerated european shade tolerant tree species to soil type and light availability. Forest Ecology and Management 188:185-195.

Moore, J. R., and D. A. Maguire. 2005. Natural sway frequencies and damping ratios of trees: influence of crown structure. Trees 19:363-373.

Mountford, E. P. 2002. Fallen dead wood levels in the near-natural beech forest at La Tillaie reserve, Fontainbleau, France. Forestry 75:203-208.

Mund, M. 2004. Carbon pools of European beech forests (Fagus sylvatica) under different silvicultural management. Dissertationsschrift, Georg-August-Universiät Göttingen.

Muth, C. C., and F. A. Bazzaz. 2002. Tree seedling canopy responses to conflicting photosensory cues. Oecologia 132:197-204.

Nadkarni, N. M. 2001. Enhancement of forest canopy research, education, and conservation in the new millennium. Plant Ecology 153:361-367.

Namvar, K., and W. Spethmann. 1986. Die heimischen Waldbaumarten der Gattung „Tilia" (Linde). Allgemeine Forst- und Jagd-Zeitung 3:42-45.

Neumann, H. H., and G. Den Hartog. 1989. Leaf area measurements based on hemispheric photographs and leaf-litter collection in a deciduous forest during autumn leaf-fall. Agricult.Forest Meteorol. 45:325-345.

Nikinmaa, E., C. Messier, R. Sievanen, J. Perttunen, and M. Lehtonen. 2003. Shoot growth and crown development: effect of crown position in three-dimensional simulations. Tree Physiology 23:129-136.

Norton, D. A., and J. Ogden. 1990. Problems with the use of tree rings in the study of forest population dynamics. Pages 284-288 in E. Cook, and L. Kairiukstis editors. Methods of dendrochronology: Applications in the environmental science. Kluwer, Dordrecht.

Okali, D. U. U. 1966. A comparative study of the ecologically related tree species Acer pseudoplatanus and Fraxinus excelsior. I. The analysis of seedling distribution. Journal of Ecology 54:129-141. 
Oliver, C. D., W. K. Clatterbuck, and E. C. Burkhardt. 1990. Spacing and stratification patterns of cherrybark oak and American sycamore in mixed, even-aged stands in the southeastern United States. Forest Ecology and Management 31:67-79.

Osada, N., H. Takeda, A. Furukawa, and M. Awang. 2002. Changes in shoot allometry with increasing tree height in a tropical canopy species, Elateriospermum tapos. Tree Physiology 22:625-632.

Otto, C. 2000. Historische Landschaftsanalyse im Nationalpark Hainich und deren Möglichkeiten der Anwendung. Diplomarbeit im Fachbereich Landschaftsnutzung und Naturschutz an der Fachhochschule Eberswalde.

Ottorini, J.-M., N. Le Goff, and C. Cluzeau. 1996. Relationships between crown dimensions and stem development in Fraxinus excelsior. Canadian Journal of Forest Reserach 26:394-401.

Ow, L. v. 1948. Über die Dürreempfindlichkeit der einzelnen Holzarten im Auwald. Allgemeine Forstzeitschrift 21:127-130.

Ozawa, H., K. Itoh, and Y. Hori. 2000. Shoot structure and dynamics of saplings and canopies of three deciduous broad-leaved trees of a coppice forest in Central Japan. Trees 14:206-214.

Pacala, S. W., and C. D. Canham. 2002. Forest models defined by field measurements: I. The design of a northeastern forest simulator. Canadian Journal of Forest Reseach 23:1980-1988.

Peters, R., and T. L. Poulson. 1994. Stem growth and canopy dynamics in a worldwide range of Fagus forests. Journal of Vegetation Science 5:421-432.

Pfeilsticker, K. H. 1983. Alarmierende Immissionsschäden an der Fichte auch in Oberschwaben. Allgemeine Forstzeitschrift 38:83-84.

Pigott, C. D., and J. P. Huntley. 1978. Factors controlling the distribution of Tilia cordata at the northern limits of its geographical range. I. Distribution in North-west England. New Phytologist 81:429-441.

Pigott, C. D., and J. P. Huntley. 1980. Factors controlling the distribution of Tilia cordata at the northern limits of its geographical range. II. History in North-west England. New Phytologist 84:145-164.

Pigott, C. D., and J. P. Huntley. 1981. Factors controlling the distribution of Tilia cordata at the northern limits of its geographical range. III. Nature and causes of seed sterility. New Phytol. 87:817-839.

Pigott, C. D. 1975. Natural regeneration of Tilia cordata in relation to forest-structure in the forest of Bialowieza, Poland. Philosophical Transactions of the Royal Society Ser.B. 270:151-179.

Pigott, C. D. 1991. Tilia cordata Miller. Biological Flora of the British Isles, Nr. 174. Journal of Ecology 79:1147-1207. 
Piutti, E., and A. Cescatti. 1999. A new detrending method for analysis of the climatecompetition relations in tree-ring sequences. Pages 249-264 in R. Wimmer, and R. E. Vetter editors. Tree-ring analysis: biological, methodological, and environmental aspects. University Press, Cambidge.

Piutti, E., and A. Cescatti. 1997. A quantitative analysis of the interactions between climatic response and intraspecific competition in European beech. Canadian Journal of Forest Reseach 27:277-284.

Pommerening, A., P. Biber, D. Stoyan, and H. Pretzsch. 2000. Neue Methoden zur Analyse und Charakterisierung von Bestandesstrukturen. Forstwissenschaftliches Centralblatt 119:62-63.

Pretzsch, H. 2002. Grundlagen der Waldwachstumsforschung. Parey, Berlin; Wien.

Pretzsch, H. 1992. Modellierung der Konkurrenz von Fichte und Buche in Rein- und Mischbeständen. Allgemeine Forst- und Jagd-Zeitung 163:203-213.

Pretzsch, H., and S. Seifert. 2000. Methoden zur Visualisierung des Waldwachstums. Forstwissenschaftliches Centralblatt 119:100-113.

Pukkala, T., T. Kuuluvainen, and P. Stenberg. 1993. Below-Canopy Distribution of Photosynthetically Active Radiation and Its Relation to Seedling Growth in A Boreal Pinus-Sylvestris Stand - A Simulation Approach. Scandinavian Journal of Forest Research 8:313-325.

Radeloff, V. C., A. M. Pidgeon, and P. Hostert. 1999. Habitat and population modelling of roe deer using an interactive geographic information system. Ecological Modelling 114:287-304.

Ratcliffe, P. R. 1987. The management of red deer populations resident in upland forests. Pages 44-53 in D. C. Jardine editor. Wildlife Management in Forests. Institute of Chartered Foresters, Edinburgh, UK.

Rauh, W. 1939. Über Gesetzmäßigkeit der Verzweigung und deren Bedeutung für die Wuchsformen der Pflanzen. Mitteilungen der Deutschen Dendrologischen Gesellschaft 52:86-111.

Remmert, H. 1991. The mosaic-cycle concept of ecosystems - an overview. Ecological Studies 85:1-21.

Rennenberg, V. H., W. Seiler, R. Matyssek, A. Gessler, and J. Kreuzwieser. 2004. European beech (Fagus sylvatica L.) - a forest tree without future in the south of Central Europe? Allgemeine Forst und Jagdzeitung 175:210-224.

Roloff, A. 1985. Auswirkungen von Immissionsschäden in Buchenbeständen. Allgemeine Forstzeitschrift 40:905-908.

Roloff, A. 2001. Baumkronen: Verständnis und praktische Bedeutung eines komplexen Naturphänomens. Ulmer, Stuttgart. 
Roloff, A. 1988. Morphologie der Kronenentwicklung von Fagus sylvatica L. (Rotbuche) unter besonderer Berücksichtigung neuartiger Veränderungen. II. Strategie der Luftraumeroberung und Veränderungen durch Umwelteinflüsse. Flora 180:297-338.

Roth, R. 1996. Der Einfluss des Rehwildes auf die Naturverjüngung von Mischwäldern. Zeitschrift für Jagdwissenschaft 42:143-156.

Rouvinen, S., and T. Kuuluvainen. 1997. Structure and asymmetry of tree crowns in relation to local competition in a natural mature Scots pine forest. Canadian Journal of Forest Reseach 27:890-902.

Röhle, H., and W. Huber. 1995. Untersuchungen zur Methode der Ablotung von Kronenradien und der Berechnung von Kronengrundflächen. Forstarchiv 56:238-243.

Röhrig, E. 1966. Mischbestände aus Edellaubbaumarten und Buche. Der Forst- und Holzwirt 3:59-64.

Röhrig, E., and N. Bartsch. 1992. Waldbau auf ökologischer Grundlage. Band I: Der Wald als Vegetationsform und seine Bedeutung für den Menschen., 6 edition. Parey, Hamburg, Berlin.

Röhrig, E., N. Bartsch, and B. v. Lüpke. 2006. Waldbau auf ökologischer Grundlage., 7 edition. Ulmer, Stuttgart.

Rubner, H. 1960. Die Hainbuche in Mittel- und Westeuropa. Pages 72-90 in Forschungen zur deutschen Landeskunde. Bundesanstalt für Landeskunde und Raumforschung, Bad Godesberg.

Rudnicki, M., U. Silins, and V. J. Lieffers. 2001. Measure of simultaneous tree sways and estimation of crown interactions among a group of trees. Trees 15:83-90.

Runkle, J. R. 1981. Gap regeneration in some old-growth forests of the Eastern United States. Ecology 62:1041-1051.

Rust, S., and A. Roloff. 2004. Acclimation of crown structure to drought in Quercus robur L. - intra- and inter-annual variation of abscission and traits of shed twigs. Basic and Applied Ecology 5:283-291.

Rust, S., and P. S. Savill. 2000. The root systems of Fraxinus excelsior and Fagus sylvatica and their competitive relationships. Forestry 73:499-506.

Sachsse, H., and W. Schwaer. 1991. Holzeigenschaften der Winterlinde (Tilia cordata Mill.). Holz als Roh- und Werkstoff 49:423-427.

Schenk, H. J. 1996. Modelling the effects of temperature on growth and persistence of tree species: A critical review of tree population models. Ecological Modelling 92:1-32.

Schipka, F. 2002. Blattwasserzustand und Wasserumsatz von vier Buchenwäldern entlang eines Niederschlagsgradienten in Mitteldeutschland. Dissertationsschrift, GeorgAugust-Universität Göttingen.

Schipka, F., J. Heimann, and C. Leuschner. 2005. Regional variation in canopy transpiration of Central European beech forests. Oecologia 143:260-270. 
Schmidt, A., and R. Hartmann. 1984. Die Abhängigkeit der neuartigen Waldschäden von Standort und Wasserversorgung im Forstamt München. Allgemeine Forstzeitschrift 39:552.

Schmidt, W. 1998. Dynamik mitteleuropäischer Buchenwälder. Kritische Anmerkungen zum Mosaik-Zyklus-Konzept. Naturschutz und Landschaftsplanung 30:242-249.

Schöne, C. 2004. Etablierung von Baumkeimlingen im Einzugsgebiet des Forschungskrans im Leipziger Auwald. Diplomarbeit im Fach Spezielle Botanik, Universität Leipzig.

Schütz, J. P. 1994. Waldbauliche Behandlungsgrundsätze in Mischbeständen. Schweiz. Z. Forstwesen 145:389-399.

Schütz, J. P. 1989. Zum Problem der Konkurrenz in Mischbeständen. Schweiz.Z.Forstwesen 140:1069-1083.

Schweingruber, F. H. 1983. Der Jahrring. Standort, Methodik, Zeit und Klima in der Dendrochronologie. Haupt, Bern; Stuttgart.

Schweingruber F. H. 1996. Tree rings and environment. Dendroecology. Haupt, Bern, Stuttgart, Wien.

Schwinning, S., and J. Weiner. 1997. Mechanisms determining the degree of size asymmetry in competition among plants. Oecologia 113:447-455.

Senn, J., U. Wasem, and O. Odermatt. 2002. Impact of browsing ungulates on plant cover and tree regeneration in windthrow areas. Forest Snow and Landscape Research 77:161170 .

Shi, H., and L. J. Zhang. 2003. Local analysis of tree competition and growth. Forest Science 49:938-955.

Shi, H., and L. J. Zhang. 2005. Local analysis of tree competition an growth. Forest Science 49:938-955.

Sorrensen, K. A., E. D. Ford, and D. G. Sprugel. 1993. A model of competition incorporating plasticity through modular foliage and crown development. Ecological Monographs 63:277-304.

Staufer, R. 1996. Waldbauliche Erfahrungen mit der Hainbuche im Forstamt Arnstein. in B. L. f. W. u. Forstwirtschaft editor. Beiträge zur Hainbuche. Bayrische Landesanstalt für Wald und Forstwirtschaft, Freising:41-45.

Sterck, F. J., and F. Bongers. 2001. Crown development in tropical forest trees: patterns with tree height and light availability. Journal of Ecology 89:1-13.

Stoll, P., and B. Schmid. 1998. Plant foraging and dynamic competition between branches of Pinus sylvestris in contrasting light environments. Journal of Ecology 86:934-945.

Stöhr, A., and R. Lösch. 2004. Xylem sap flow and drought stress of Fraxinus excelsior saplings. Tree Physiology 24:169-180. 
Suzuki, A. 2002. Influence of shoot architectural position on shoot growth and branching patterns in Cleyera japonica. Tree Physiology 12:885-890.

Szwagrzyk, J. 1997. Modelling competition among trees in mixed stands of complex structure. Krakow.

Tal, O. 2006. Comperative flowering ecology of Fraxinus excelsior, Acer platanoides, Acer pseudoplatanus and Tilia cordata in the canopy of Leipzig's floodplain forest. Dissertationsschrift, Universität Leipzig.

Tapper, P. G. 1996. Tree dynamics in a succetional Alnus-Fraxinus woodland. Ecological Modelling 19:237-244.

Tarppeiner, J. C., D. Lavender, J. Walstad, R. O. Curtis, and D. S. DeBell. 1997. Silvicultural systems and regeneration methods: Current practices and new alternatives. Pages 151164 in K. A. Kohm, and J. F. Franklin editors. Creating a forestry for the 21st Century: The science of ecosystem management.

Tremmel, D. C., and F. A. Bazzaz. 1993. How neighbor canopy architecture affects target plant performance. Ecology 74:2114-2124.

Ueckermann, E. 1988. Der Rehwildabschuss. 6 edition. Paul Parey, Hamburg.

Vester, H. 1997. The trees and the forest. The role of tree architecture in canopy development; a case study in secondary forests (Araracuara, Colombia). Wageningen, Ponsen \& Looijen.

Vester, H. 2002. Analyse der Standraumökonomie von Einzelbäumen auf langfristig beobachteten Versuchsflächen - Methoden, Programmentwicklung und erste Ergebnisse. Forstwissenschaftliches Centralblatt 121:335-348.

Wagner, S. 1999. Ökologische Untersuchungen zur Initialphase der Naturverjüngung in Eschen-Buchen-Mischbeständen. Sauerländer's Verlag, Frankfurt am Main.

Weber, G. 2003. Das Ende der Ära 'Waldumbau'? Das Blatt - Mitarbeiterinformationen der Thüringer Landesforstverwaltug 10:9-10.

Weitland, J. 1960. Jahrringchronologische Untersuchungen an Laubbaumarten Norddeutschlands. Mitteilungen der Bundesforschungsanstalt für Forst- und Holzwirtschaft 48:1-122.

Wensel, L., Meerschaert W. \& Biging G.S. 1987. Tree height and diameter growth models for Northern California Conifers. Hilgardia, University of California 55[8].

Wenzel, K. F. 1987. Höhenzuwachs-Analysen zur Diagnose von Immissionswirkungen. Allgemeine Forstzeitschrift 38:342-347.

Wiermann, C. A., and C. D. Oliver. 1979. Crown stratification by species in even-aged mixed stands of Douglas-fir - western hemlock. Canadian Journal of Forest Research 9:1-9.

Wohlgemuth, T., M. Bürgi, C. Scheidegger, and M. Schütz. 2002. Dominance reduction of species through disturbance - a proposed management principle for Central European forests. Forest Ecology and Management 1-15. 
Worbes, M., S. Bonn, and T. Riemer. 1995. Methoden zur Erfassung von Zuwachsverlusten und mögliche Einflußfaktoren auf das Jahresringbild von Bäumen in geschädigten Waldbeständen. Forstwissenschaftliches Centralblatt 114:313-325.

Worbes, M., M. Hofmann, and A. Roloff. 1992. Wuchsdynamik der Baumschicht in einem Seggen-Kalkbuchenwald in Nordwestdeutschland (Huckstein). Dendrochronologia 10:91-106.

Yokozawa, M., Y. Kubota, and T. Hara. 1996. Crown architecture and species coexistence in plant communities. Annals of Botany 78:437-447.

Yoshida, T., and T. Kamitani. 2000. Interspecific competition among three canopy-tree species in a mixed-species even-aged forest of Central Japan. Forest Ecology and Management 137:221-230.

Yoshida, T., and T. Kamitani. 1997. The stand dynamics of a mixed coppice forest of shadetolerant and intermediate species. Forest Ecology and Management 95:35-43. 


\section{Danksagung}

Ich danke allen, die zum Entstehen dieser Arbeit beigetragen haben: Christoph Leuschner für seine fachliche Unterstützung vor allem in der Anfangs- und Endphase und für den Freiraum in der Zwischenzeit; der Deutschen Bundesstiftung Umwelt für die finanzielle Unterstützung und die vielen wertvollen Erlebnisse und Kontakte, die durch die Seminare und Treffen zustande gekommen sind und mich sicher noch lange begleiten werden; der Nationalparkverwaltung des Nationalparks Hainich und besonders Herrn Großmann für seine Hilfbereitschaft; Herrn Worbes und Esther Fichtler für die fachliche Betreuung der dendroökologischen Untersuchungen, Kai und Iris Frech für die GIS-Hilfe, Catharina Meinen und Nicole Burgdorf für die schöne Zusammenarbeit im Hainich, Ophir Thal für seine ständige Diskussionsbereitschaft und Kooperation, die meine Arbeit sehr bereichert hat, Hermann Jungkunst und Herrn Bareth für die Erstellung der Deutschlandkarte; dem Institut für Waldbau und dem Institut für Waldinventur und Waldwachstum für das zu Verfügung Stellen des Winkelmessers und der Totalstation; Mechthild Stange, Dirk Deilke, Ulrich Werder, Heinz Coners, Michael Würfel und der FFGH für die unermüdliche Hilfe bei den Freilandarbeiten, ohne die ich sicher noch einmal drei Jahre gebraucht hätte; Holger Buschmann, Sandra Korn, Heinz Coners, Boris Rewald und Michael Würfel für die Korrekturen und konstruktiven Verbesserungsvorschläge; Sandra, Jasmin und Cathi für die in jeder Konstellation schöne Zimmergemeinschaft; der Arbeitsgruppe für die ständige Hilfsund Diskussionsbereitschaft; allen Nonnis und besonders Lui und Thorsten für die fröhliche inter- und extradisziplinäre Kommunikation; Wulf für sein Vertrauen und seine Unterstützung in jeder Hinsicht; Mimi für ihr Staunen und ganz besonders danke ich meinem Sohn Aaron für seine Geduld mit mir! 
Geboren am:

in:

Eltern:

Staatsangehörigkeit:

Bildungsgang: 1981

1985

1986

1994

1994 - 1995

$1995-2000$

2001

2001

2002

$2002-2003$

2003 - 2006 Stipendium der Deutschen Bundesstiftung Umwelt
Niedersächsischen Graduiertenförderungsgesetz

02.07. 1975

\section{Bielefeld}

Heide (geb. Brimer) und Dr. med. E. Wulf-Eberhardt Frech

Deutsch

Grundschule Manderscheid (Eifel)

Peter-Wust-Gymnasium Wittlich

Freie Waldorfschule Trier

Abitur

Freiwilliges soziales Jahr in Iasi (Rumänien)

Fächern Biologie und Germanistik an der Universität Kassel

Abgabe der Examensarbeit im Fachgebiet Ökologie

Erstes Staatsexamen für das Lehramt an Gymnasien

Vorbereitung des Promotionsvorhabens an der Universität

Göttingen, Albrecht-von-Haller-Institut für Pflanzenwissen-

schaften, Abteilung Ökologie und Ökosystemforschung

Promotionsstipendium der Universität Göttingen nach dem 